\title{
NOVEL RF MEMS TUNABLE CAPACITORS
}

by

Zewdu Hailu

Bachelor of Science Arbaminch University, Arbaminch, Ethiopia, 2004

Master of Science, Addis Ababa University, Technology Faculty, Ethiopia 2008

\author{
A dissertation \\ presented to Ryerson University \\ in partial fulfillment of the \\ requirements for the degree of
}

Doctor of Philosophy

in the program of

Mechanical and Industrial Engineering

Toronto, Ontario, Canada, 2014

(C) Zewdu Hailu June 2014 


\section{Author's Declaration for Electronic Submission of a Dissertation}

I hereby declare that I am the sole author of this dissertation. This is a true copy of the dissertation, including any required final revisions, as accepted by my examiners.

I authorize Ryerson University to lend this dissertation to other institutions or individuals for the purpose of scholarly research

I further authorize Ryerson University to reproduce this dissertation by photocopying or by other means, in total or in part, at the request of other institutions or individuals for the purpose of scholarly research.

I understand that my dissertation may be made electronically available to the public. 


\section{Abstract \\ Novel RF MEMS Tunable Capacitors \\ Doctor of Philosophy}

June 2014

\section{Zewdu Hailu}

\section{Mechanical and Industrial Engineering Department, Ryerson University}

Current tunable devices such as filters, impedance matching networks and oscillators have problems that degrade their performance at high microwave frequencies. Tuning ratios and quality factors are the major problems associated with semiconductor based tuning components.

This thesis presents the design, fabrication and testing of two novel RF MEMS tunable capacitors. The first tunable capacitor is designed using electrostatic repulsive-force actuators which produce an upward movement of the moving plate of a tunable capacitor. The repulsiveforce actuator is free of pull-in effect and capable of reaching large displacement.

Gap increasing tunable capacitors with areas of $162 \mu \mathrm{m} \times 220 \mu \mathrm{m}$ and $300 \mu \mathrm{m} \times 302 \mu \mathrm{m}$ are developed using electrostatic repulsive-force actuators. The capacitances are calculated using simulations and maximum tuning ratios of $438.5 \%$ and $230 \%$ are obtained for a parallel and inclined plate designs, respectively, with capacitance-voltage linearity of $96.28 \%$ and $95.14 \%$, respectively, in the presence of RF voltage.

The second tunable capacitor is developed using residual stress gradient based vertical combdrive actuator. Conventional vertical comb-drive actuators need two vertical comb fingers, i.e., one for the fixed and one for the moving comb. MetalMUMPs process provides a $20 \mu \mathrm{m}$ thick nickel layer which is subject to residual stress gradient along its thickness. Using the residual stress gradient two curve-up beams are devised to bend out of plane and upward. A moving plate 
is connected between the middles of the curve-up beams through supporting springs and is raised above the substrate. The moving fingers are connected to opposite sides of the moving plate. The fixed comb-drive fingers are anchored to the substrate. When a voltage is applied, the moving fingers move down towards the fixed fingers. As a result, the capacitance between the moving fingers and the fixed fingers change. Prototypes are fabricated to verify the working principles of this novel actuator using the MetalMUMPs process. Tunable capacitors based on this actuator are experimentally analyzed. Quality factors of $106.9-162.7$ at $0.8 \mathrm{GHz}$ and $42.4-51.9$ at $1.24 \mathrm{GHz}$ are obtained over actuation voltage of $0-100 \mathrm{~V}$. An optimal design of the tunable capacitors achieved a tuning ratio of $194.4 \%$ at $162.5 \mathrm{~V}$ with linearity of $97.84 \%$. 


\section{Acknowledgements}

I am thankful to my supervisors Prof. Siyuan He and Prof. Ridha Ben Mrad for their tremendous support throughout the thesis work. Their comments and ideas have provided invaluable input to the completion of this work.

I am grateful to Canadian Microelectronics Corporation and the respective personnel for the fabrication of prototypes used in the research work.

I am thankful to Mr. James Dietrich, RF Lab Engineer of Advanced RF Systems Lab of University of Manitoba, for his willingness to test some of my capacitors.

I would like to thank my previous lab mates James Chong and Soonho Park for their friendly help at some point in the past.

I would like to say thank you to Joseph Amankrah, Qiang Li, and Chao Ma for their technical support at some stages of the research work.

Finally, I like to thank my brother, Dr. Getu Hailu, for all his help and advice. 
Dedication

To my father (HWG) 
$\begin{array}{lll}\text { Contents } & \text { Page }\end{array}$

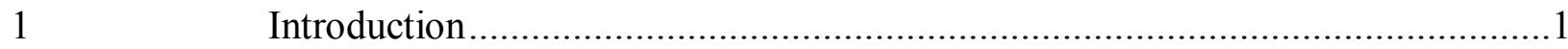

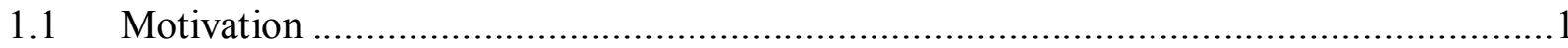

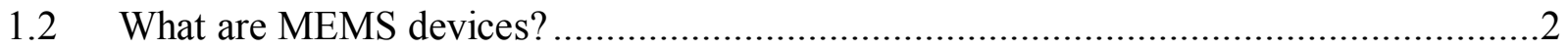

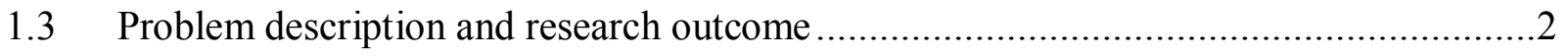

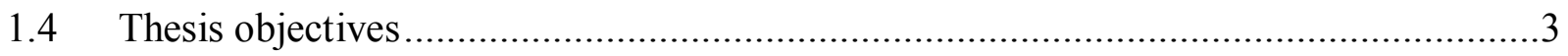

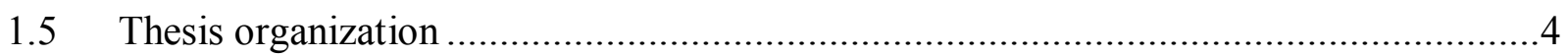

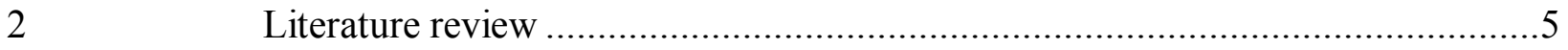

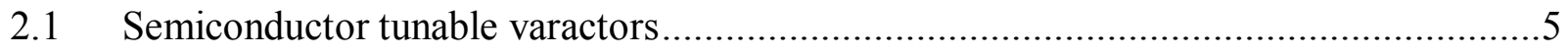

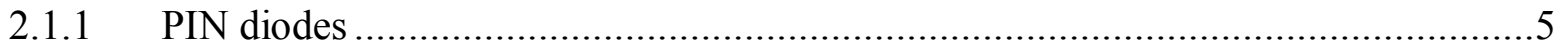

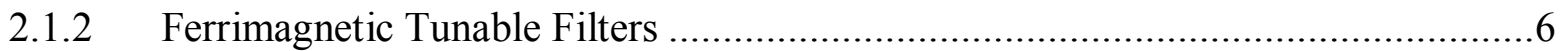

2.2 RF MEMS tunable capacitors review ………….....................................................

2.2.1 Parallel plate electrostatic actuator tunable capacitors .........................................

2.2.2 Parallel plate tunable capacitors with different gaps ...........................................

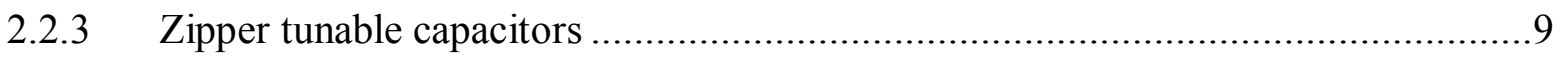

2.2.4 MEMS bridges as switchable capacitor ..........................................................

2.2.5 Flip-chipped digitally controlled cascaded capacitors .........................................10

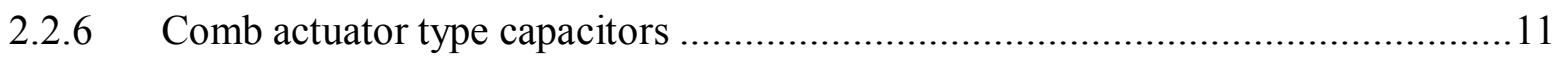

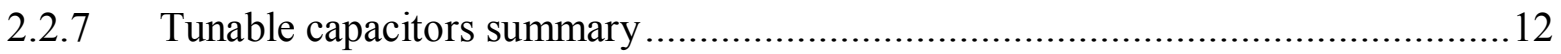

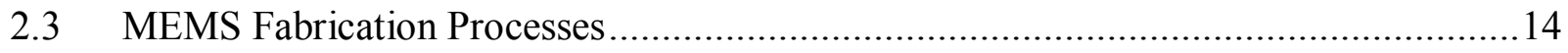

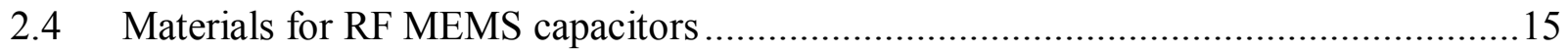

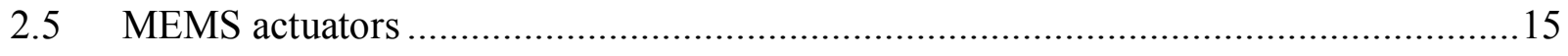

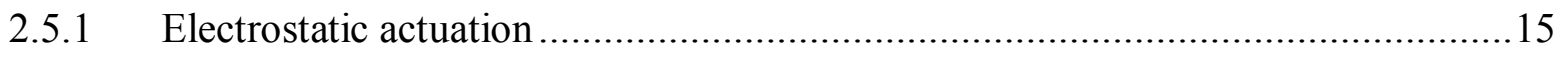

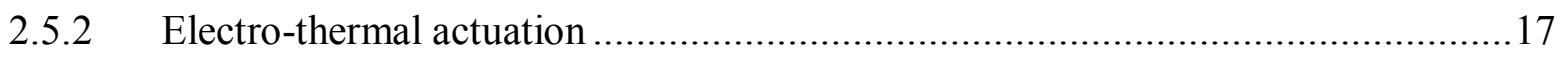




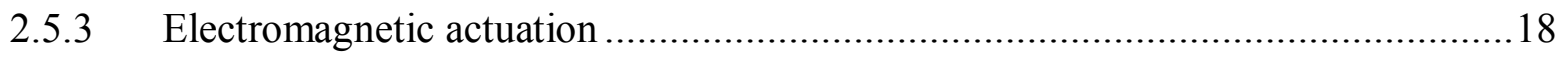

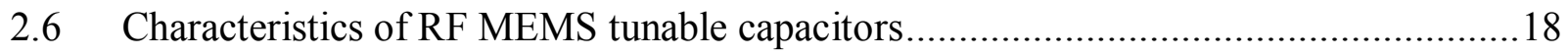

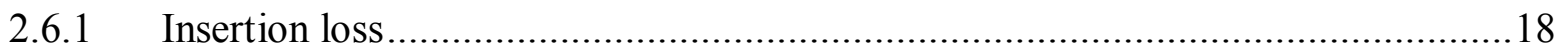

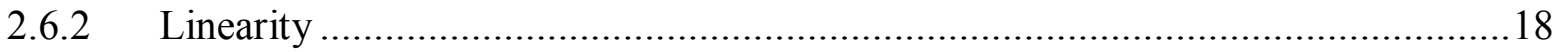

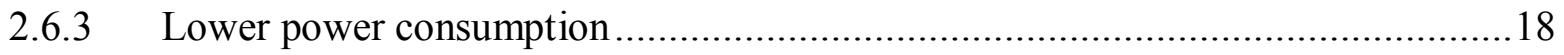

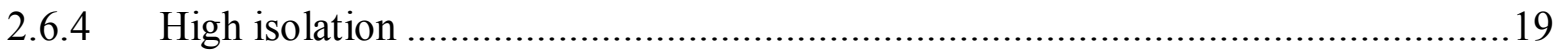

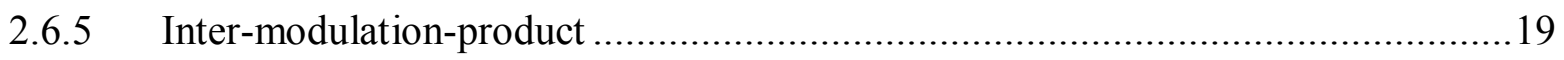

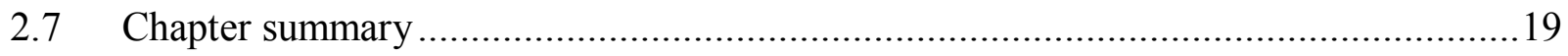

3 A Repulsive Electrostatic Force Driven RF MEMS Tunable Capacitor..............20

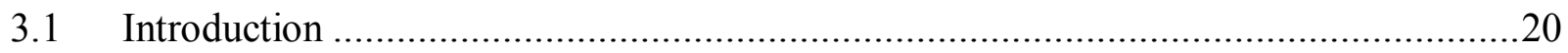

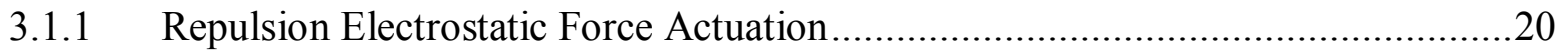

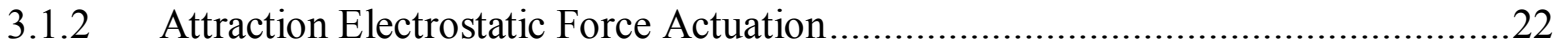

3.1.3 Repulsive electrostatic force from capacitance ..............................................23

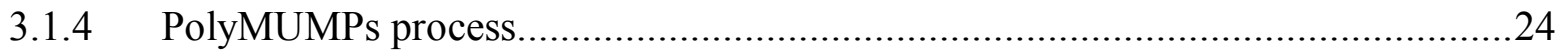

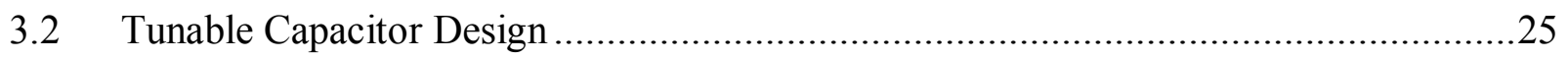

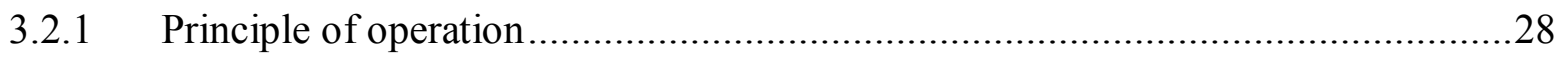

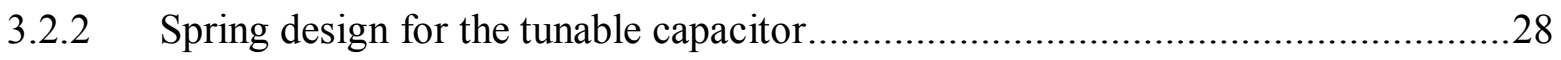

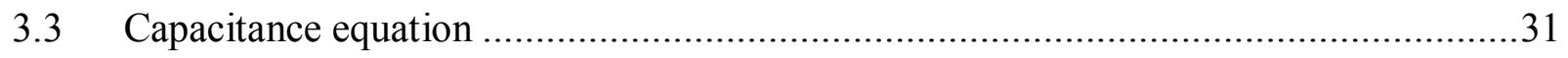

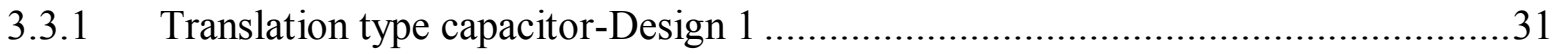

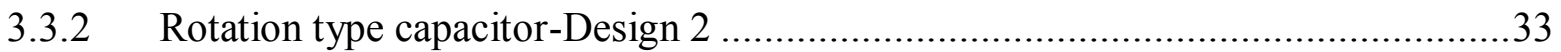

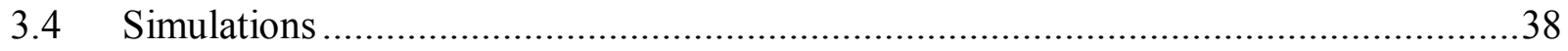

3.5 RF power and tuning ratio of repulsive capacitors ....................................................42

3.5.1 Interaction of RF power and actuation voltage …….........................................42

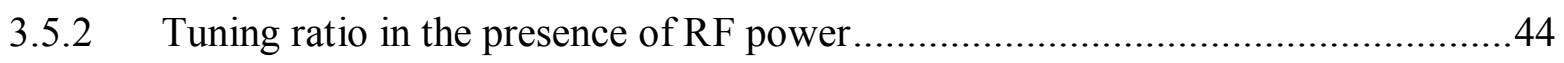

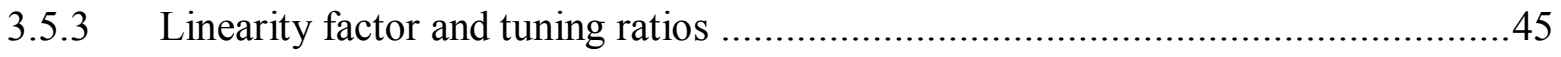




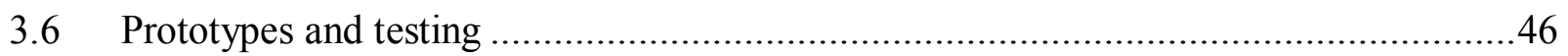

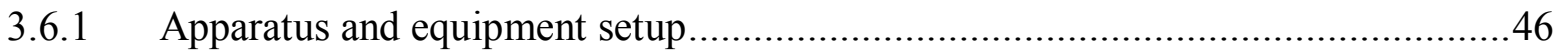

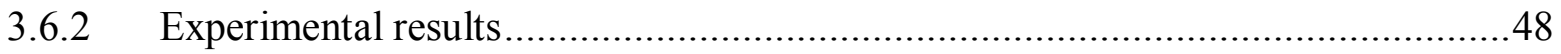

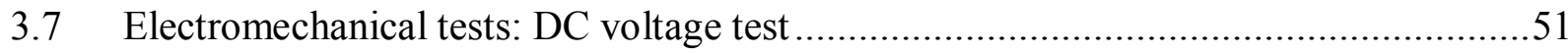

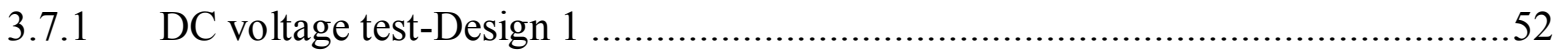

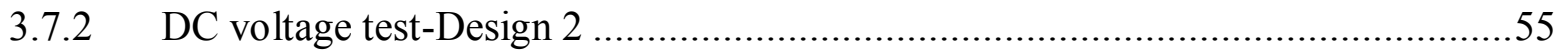

3.8 Analysis of attraction force induced on the RF electrodes.........................................57

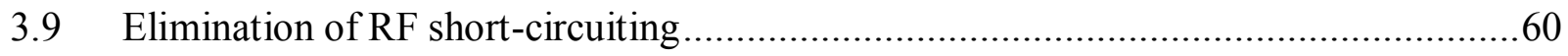

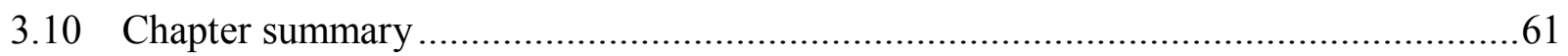

4 A Novel Vertical Comb-Drive Actuator Using a Single Layer of MetalMUMPs

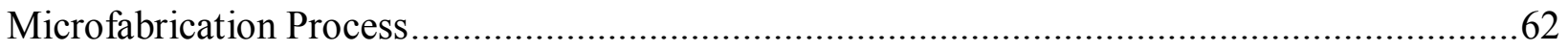

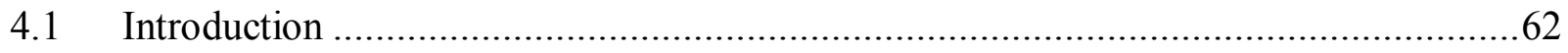

4.2 Residual stresses and stress gradients in micro films ..............................................63

4.3 Residual stresses in MetalMUMPs fabrication process ……........................................64

4.4 Micro-fabrication process-MetalMUMPs process......................................................66

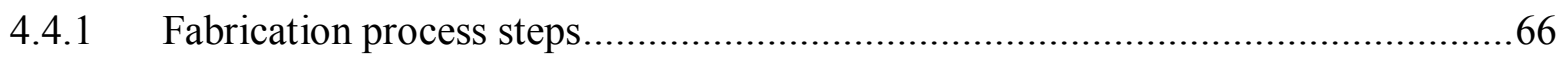

4.4.2 Material properties and Design specifications .................................................67

4.4.3 Simulation of MetalMUMPs clamped-clamped and cantilever beams ..................68

4.5 Design principle of vertical comb-drive actuator ....................................................70

4.6 Actuator layout, 3D models and simulations ………...............................................72

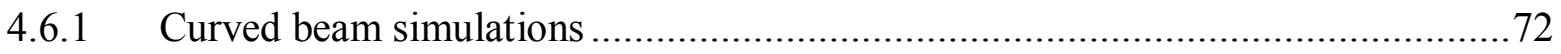

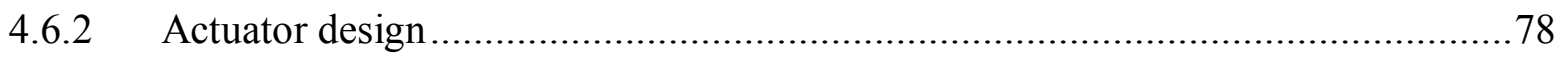

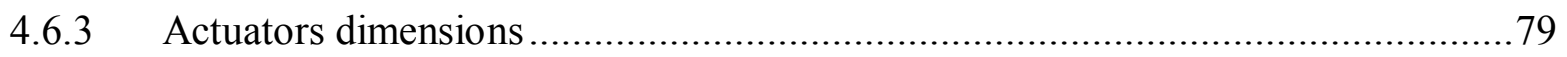

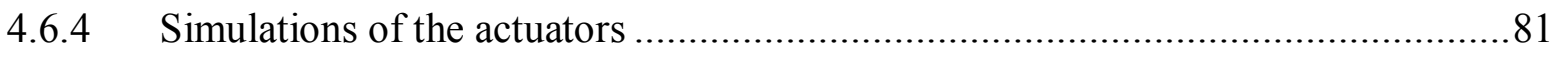

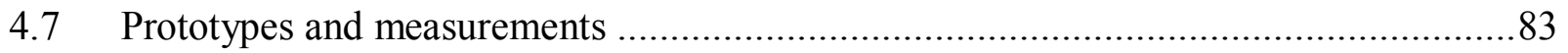


4.7.1 Measurement without DC voltage .................................................... 83

4.7.2 Measurement with DC voltage applied ...................................................95

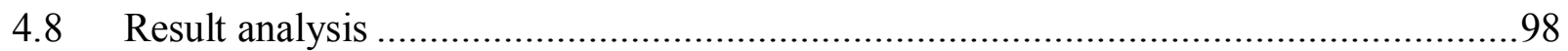

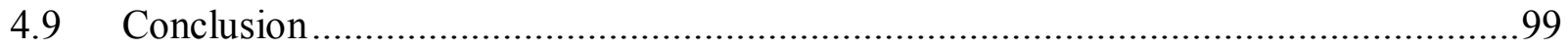

5 Application of the Vertical Comb Actuator to MEMS Tunable Capacitor ....... 101

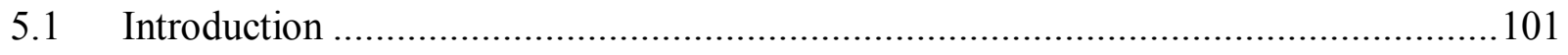

5.2 Design of vertical comb drive tunable capacitor .............................................. 102

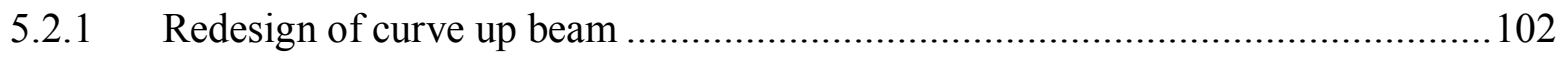

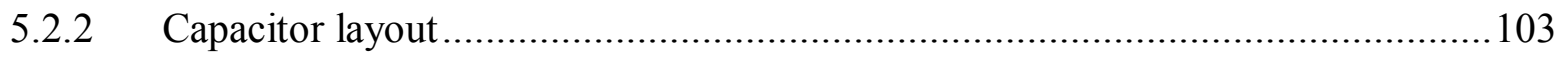

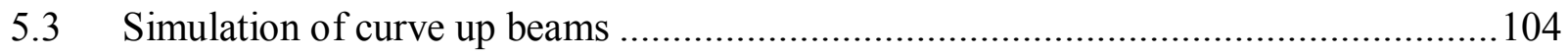

5.4 Capacitor 3D models and simulations ......................................................... 107

5.5 Effect of geometric parameters on the maximum heights................................ 111

5.6 Prototypes measurement and verification ................................................. 112

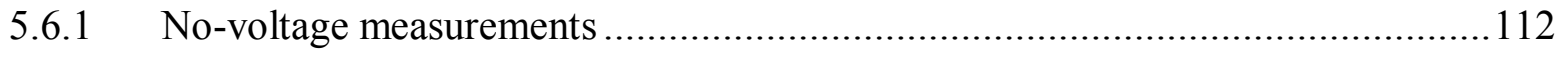

5.6.2 Simulated Vs. measured height ............................................................. 118

5.6.3 Electromechanical measurements-voltage test.......................................... 119

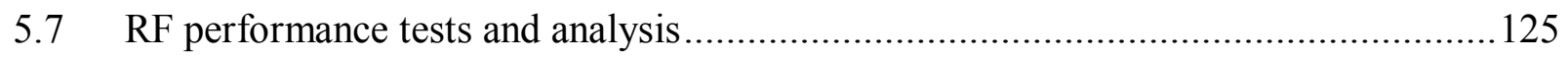

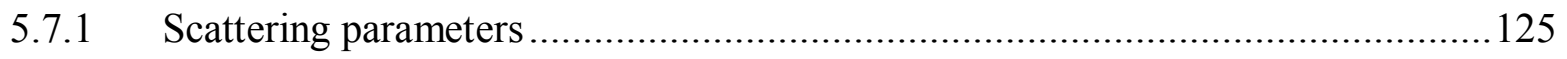

5.7.2 S-parameter conversion equations ................................................. 125

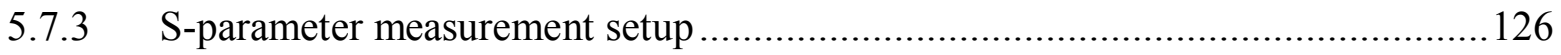

5.7.4 Capacitance extraction and quality factor calculation .................................. 129

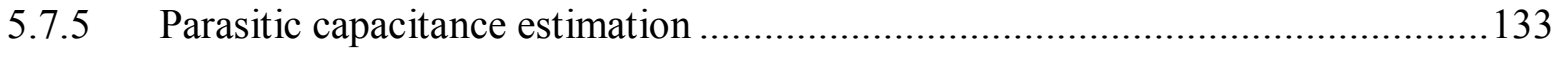

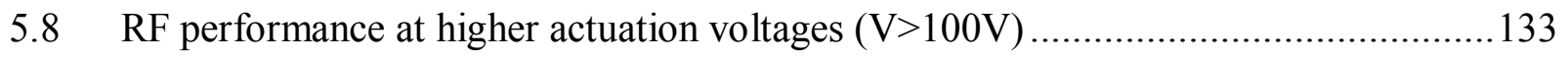

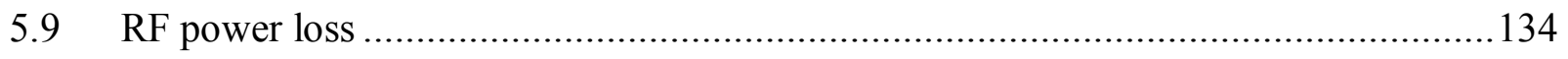

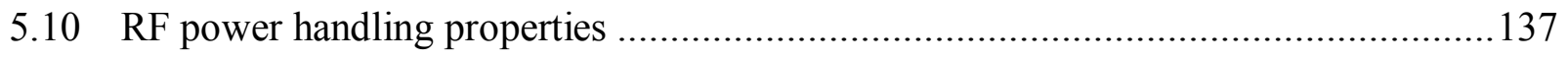




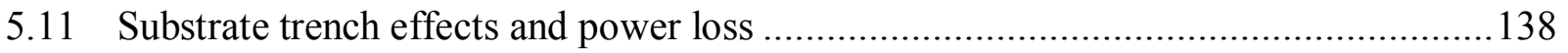

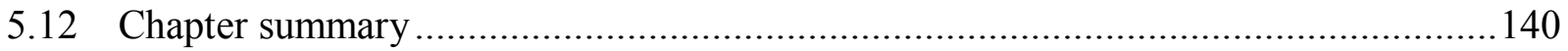

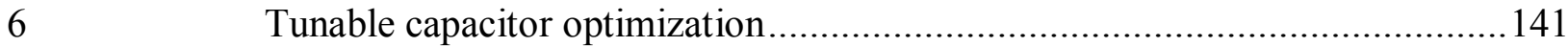

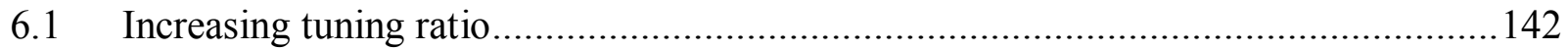

6.1.1 Idea of increasing tuning ratio ............................................................ 142

6.1.2 Optimization of finger size, number and gap ........................................ 143

6.1.3 Influence of electrostatic forces on the outer fingers ................................... 144

6.1.4 Tuning ratio of the optimized capacitor .............................................. 148

Optimization of pads and strips ............................................................... 151

6.3 Optimization of tuning ratio and quality factors by trench .............................. 153

6.4 Advantages of novel tunable capacitor ................................................. 155

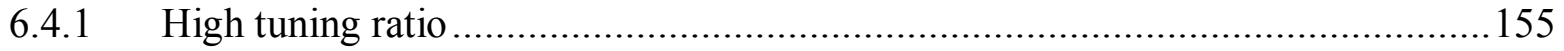

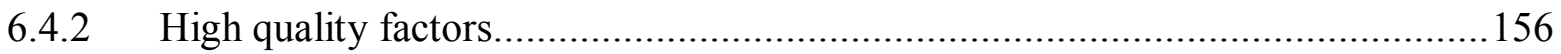

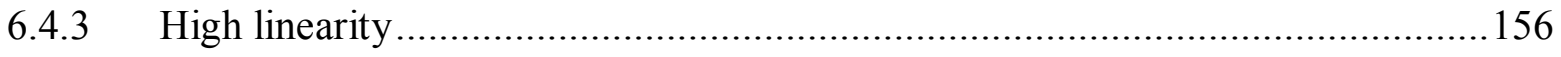

6.4.4 High power handling ......................................................................... 156

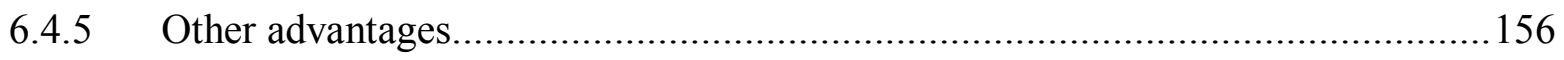

6.4.6 Comb drive actuators .......................................................................... 157

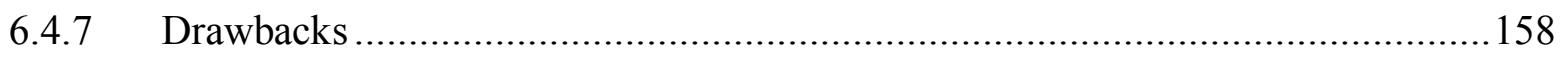

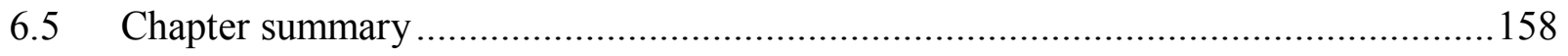

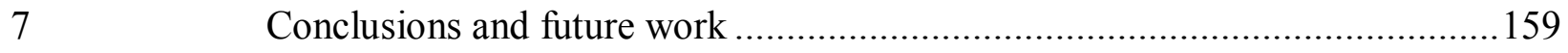

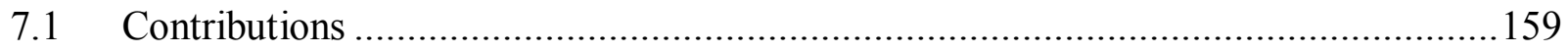

7.1.1 Electrostatic repulsive- force actuated tunable capacitor............................... 159

7.1.2 Novel residual stress gradient based vertical comb-drive actuator and its

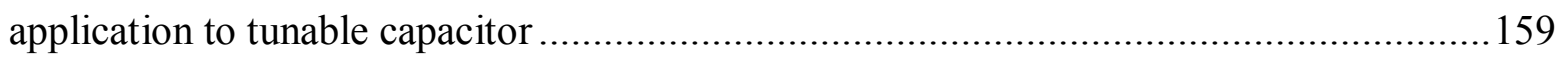

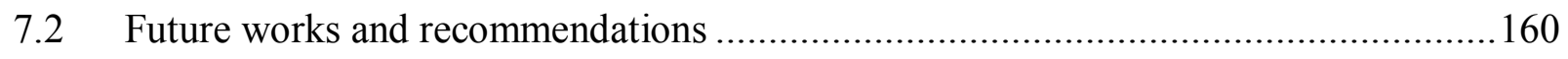


7.2.1 Tunable capacitors driven by the repulsive-force actuator ............................. 160

7.2.2 Tunable capacitors driven by the novel vertical comb actuator ........................ 161

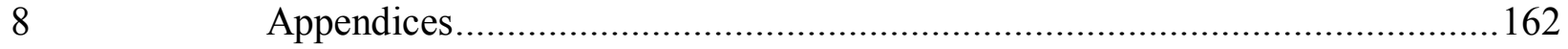

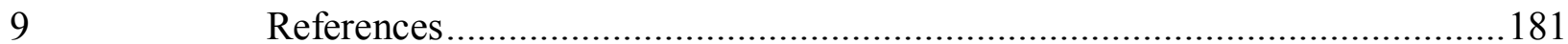


CMOS----------------------- Complementary Metal-Oxide Semiconductor

CVD Chemical Vapor Deposition

$\mathrm{DC}$ Direct Current

$\mathrm{dB}$ -Decibel

$\mathrm{dBm}-$ -Decibel Millimeter

DRIE -Deep Reactive Ion Etching

FEA-Finite Element Analysis

FET Field effect transistors

FOV Field of View

GND--------------Ground

HEMT High Electron-Mobility Transistors

LF-Linearity Factor

LPCVD -Low Pressure Chemical Vapor Deposition

MEM -Microelectromechanical

MEMS Microelectromechanical systems

MIM -Metal Insulator Metal

MOSFET Metal-Oxide Semiconductor Field effect transistors MUMPs Multi User MEMS Processes

pHEMT Pseudo High Electron-Mobility Transistors

PVD physical vapor deposition

$\mathrm{PN}$ -Positive Negative

PIN Positive Intrinsic Negative

Q-----------------------Quality Factor

$\mathrm{RF}$ -Radio Frequency

RMS Root Mean Square

SAW Surface Acoustic Wave

SEMScanning Electron Microscope $\mathrm{S}$ Scattering parameters VNA-Vector Network Analyzer

YIGYttrium-Iron-Garnet 


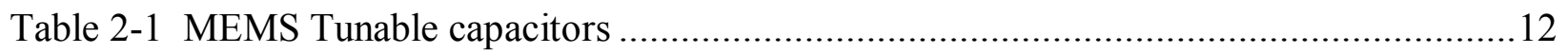

Table 3-1 Layer name and lithography levels of PolyMUMPs process [62] …….....................24

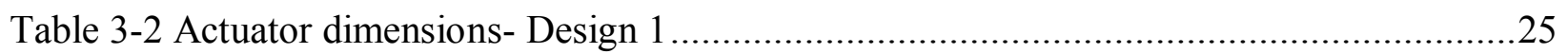

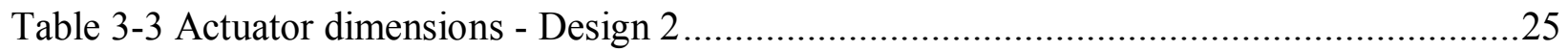

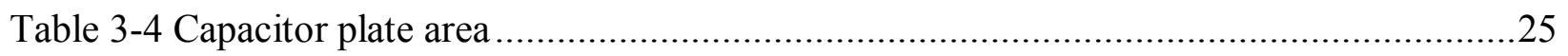

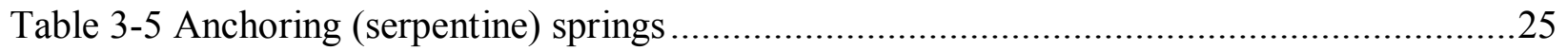

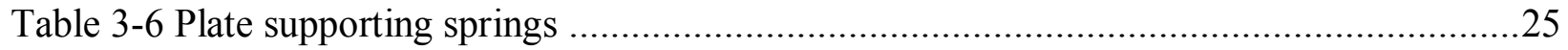

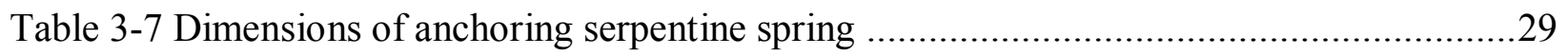

Table 3-8 Simulated spring constant of the serpentine spring.................................................

Table 3-9 Dimensions of the moving fingers and the corresponding stiffness .............................31

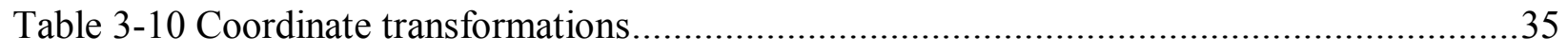

Table 3-11 Simulation results--Design 1 without RF power.....................................................39

Table 3-12 Simulation results--Design 2-without RF power .....................................................39

Table 3-13 Simulated induced voltages and electrostatic forces on the RF electrodes .................58

Table 3-14 Simulated induced voltages and electrostatic forces on the RF electrodes ................60

Table 4-1 MetalMUMPs material properties $[81,101]$..........................................................6

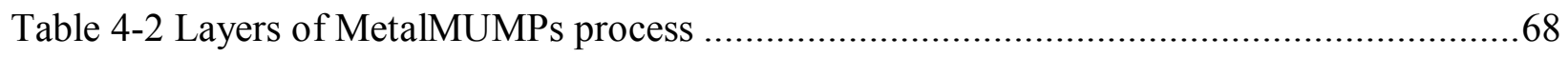

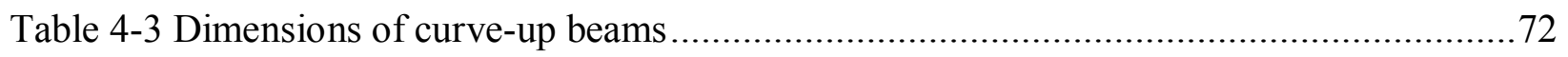

Table 4-4 Simulated and measured heights of moving finger/plate ...........................................75

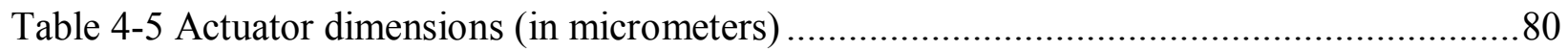

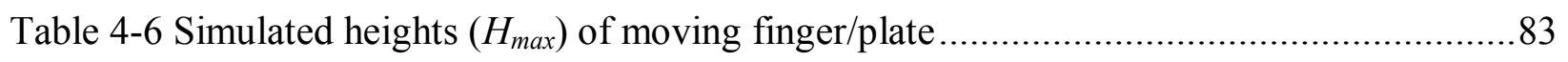

Table 4-7 Error percentage between measured and simulated deflections ..................................86

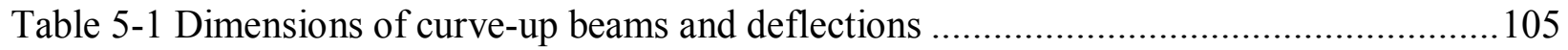

Table 5-2 Geometric parameters of tunable capacitor .............................................................. 107

Table 5-3 Simulated maximum height $\left(H_{\max }\right)$ of capacitors...................................................... 109

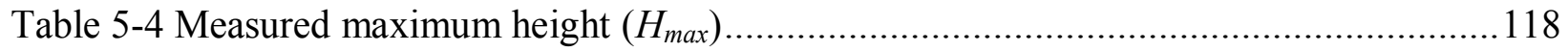

Table 5-5 Electromechanical measurements-change in displacements .....................................120

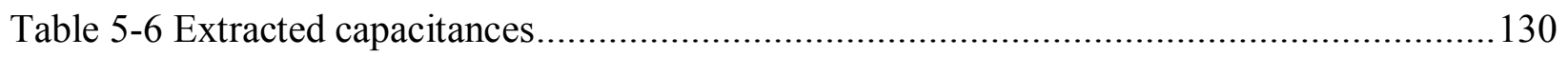

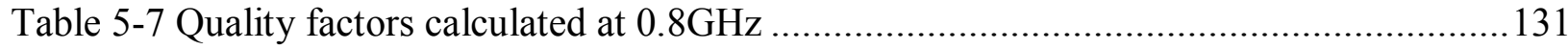

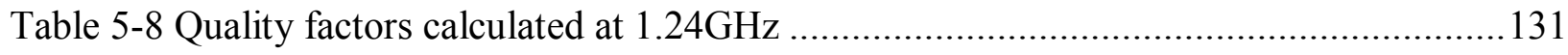


Table 5-9 Areas of trenches under the capacitor fingers and plates ................................. 139

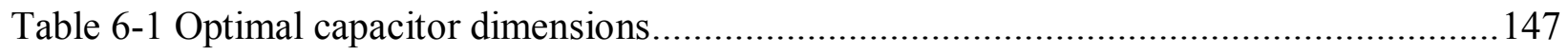

Table 6-2 Summary of optimal width of the outer moving fingers .................................. 148

Table 6-3 Capacitances of optimized tunable capacitor.................................................... 150

Table 6-4 Summary of tuning ratio based on optimized finger, strips, pads and anchor sizes ... 155 
Figure 2-1 Circuit representation and layers of PIN diodes [15] .......................................5

Figure 2-2 Schottky diodes and a schematic showing their construction ..............................6

Figure 2-3 Traditional parallel plate electrostatic actuator tunable capacitor [20] .....................7

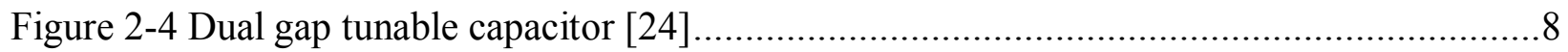

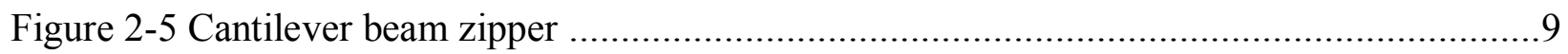

Figure 2-6 RF MEMS switchable capacitor [25] cross section view (left) and top view (right) ....9

Figure 2-7 Digitally controllable capacitors developed using flip chip process [26] .................10

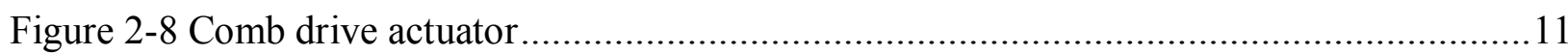

Figure 2-9 SEM image of $40 \mu \mathrm{m}$ thick tunable capacitor with comb drive actuator (left) and the schematic diagram representation (right) (R. L. Borwick et al. $[30,31])$.............................11

Figure 2-10 Illustration of the basic process flow in surface micromachining [50] ..................14

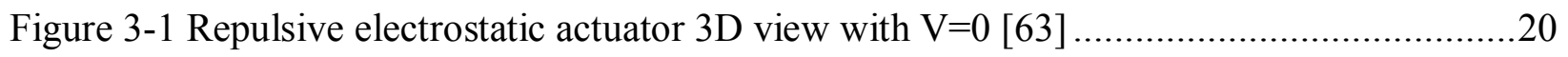

Figure 3-2 Illustrates electrostatic repulsive force actuator with $\mathrm{V} \neq 0$ [63]..........................21

Figure 3-3 Actuation mechanism for repulsive electrostatic force [63] ...............................21

Figure 3-4 2D representation of repulsive electrostatic fields [68] ...................................22

Figure 3-5 Actuation mechanism for attraction electrostatic force [68] ...............................22

Figure 3-6 2D representation of attractive electrostatic fields [68] ......................................22

Figure 3-7 Illustration of the capacitance between the electrodes of repulsive actuator .............23

Figure 3-8 A schematic of the layers in PolyMUMPs process [62] .....................................24

Figure 3-9 Translation type tunable capacitor (Design-1) ....................................................26

Figure 3-10 Rotation type tunable capacitor (Design-2).............................................27

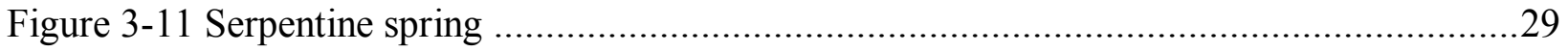

Figure 3-12 Simulated displacement of the tip of the anchoring springs at $1 \mu \mathrm{N}$ and $4 \mu \mathrm{N}$ forces 30

Figure 3-13 Deformation of repulsive actuator ............................................................... 31

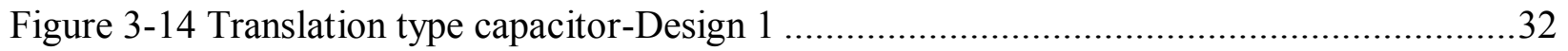

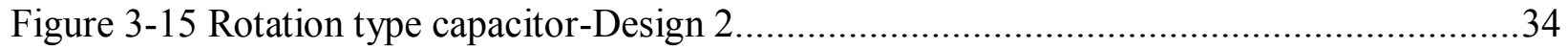

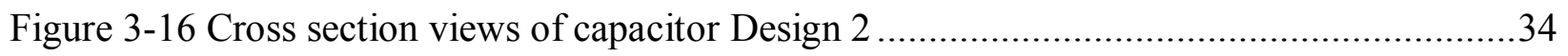

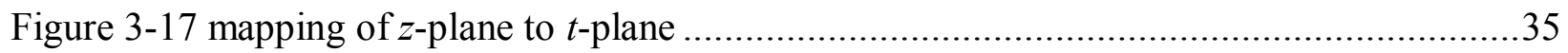

Figure 3-18 Simulated capacitance vs. voltage for Design 1 and design 2 ............................40

Figure 3-19 Displacement vs. voltage for Design 1 and Design 2 ...................................40 
Figure 3-20 Tuning of capacitor-Design 1 41

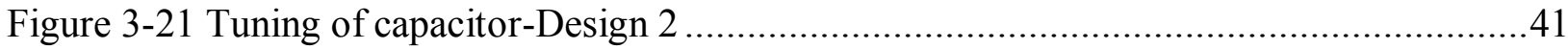

Figure 3-22 Simulated capacitor of Design 1at 1V DC RF equivalent voltage .......................43

Figure 3-23 Simulated capacitance Vs actuation and RFDC equivalent voltages-Design 1 .......44

Figure 3-24 Simulated capacitance Vs actuation and RFDC equivalent voltages -Design 2 .......45

Figure 3-25 (a) Complete setup for DC test (b) Close view of probes and sample prototype ......47

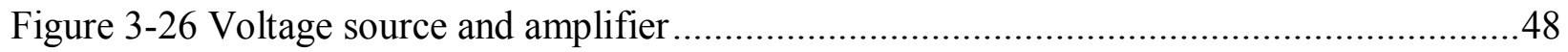

Figure 3-27 Images of capacitors: (a) Design 1 and (b) Design 2 .....................................49

Figure 3-28 Moving plate measurement: no-voltage (a) Surface map and (b) Profile plot .........49

Figure 3-29 Actuator fingers measurement: no-voltage ............................................50

Figure 3-30 Design 2 (a) Surface map and (b) Profile plot ...............................................51

Figure 3-31 Design 2 Actuator (a) surface map and (b) profile plot ....................................51

Figure 3-32 Design 1(a) Surface map and (b) Profile plot at 25V actuation ...........................52

Figure 3-33 Design 1 (a) Surface map and (b) Profile plot at 100V actuation..........................53

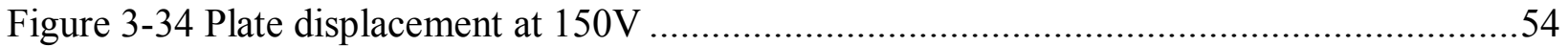

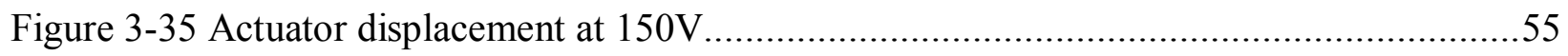

Figure 3-36 Design 2 (a) Surface map and (b) Profile plot at 100V actuation..........................56

Figure 3-37 Measured displacement of the moving plate at 125V-Design 2 .........................56

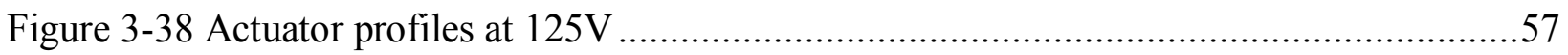

Figure 3-39 Cross-section view of the unaligned fingers and capacitor plates .........................58

Figure 3-40 Field stream lines at 25V-wtih RF electrode floating ......................................59

Figure 3-41 Proposed techniques with ground loop around the RF electrode .........................59

Figure 3-42 Hole and dimple on capacitor plates .........................................................61

Figure 4-1 Types of stresses on a cantilever beam .....................................................63

Figure 4-2 Residual stress gradient deformed clamped-clamped beam................................65

Figure 4-3 Residual stress gradient deformed cantilever beam .........................................65

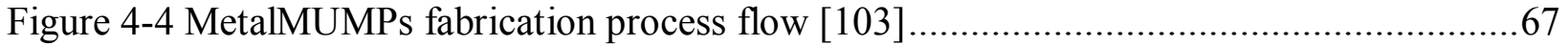

Figure 4-5 Clamped-clamped beam deformations using residual stress gradient ....................69

Figure 4-6 Cantilever beam deformations using residual stress gradient ............................69

Figure 4-7 Clamped-Clamped anchored using box-type springs ....................................69

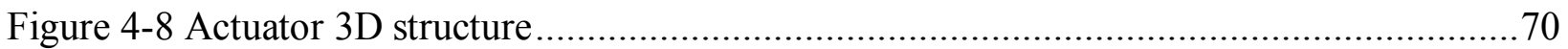




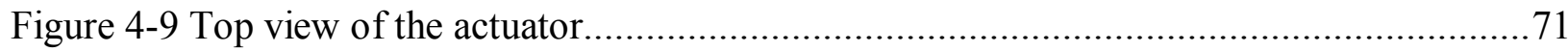

Figure 4-10 Anchoring springs (a) rectangular (b) serpentine .........................................73

Figure 4-11 Simulated beam of clamped-clamped beam .............................................. 74

Figure 4-12 Maximum deflection of curve-up beams vs. length..........................................74

Figure 4-13 Plots of shapes of curved-up beams B1-B7 .....................................................76

Figure 4-14 Polynomials fitting of the deformation of beam B7 .................................... 77

Figure 4-15 Polynomial fitting of the deformation of beam B1 ........................................77

Figure 4-16 Actuator 2D layouts with rectangular anchoring spring ....................................79

Figure 4-17 Actuator 3D models with (a) complete structure (b) trench in substrate .................79

Figure 4-18 3D maps of analysis results of actuator after stress gradient deformation...............82

Figure 4-19 (a) Chip 1(A1, A2, A3, A4, A5) and (b) Chip 2 (A6, A7) ................................84

Figure 4-20 SEM micrograph of Actuator, A7, (a) complete view and (b) magnified view ........85

Figure 4-21 Vertical height $H_{\max }$ between moving fingers and fixed fingers .........................86

Figure 4-22 Actuator A6 moving fingers not raised above the substrate...............................87

Figure 4-23 Curve-up beam measurement (a) Surface map (b) profile plot ............................89

Figure 4-24 Fixed fingers (a) Surface map and (b) profile plot .........................................90

Figure 4-25 Moving fingers (a) Surface map and (b) profile plot ...................................91

Figure 4-26 Heights of fingers above trench (a) Moving and fixed fingers (b) profile plot .........92

Figure 4-27 Heights of fingers above the trench (a) Middle section and (b) tip of moving fingers

Figure 4-28 Profile showing the bending of the tips of the (a) moving fingers (b) fixed fingers 94 Figure 4-29 Measurement at 0V (a) Surface map and (b) profile plot ..................................96

Figure 4-30 (a) Surface map at 200V and (b) Profile plot at 200V ......................................97

Figure 5-1 Clamped-clamped (a) non-deformed and (b) deformed gradient (curve-up beam)... 102

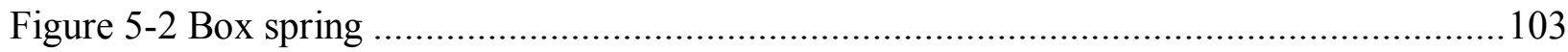

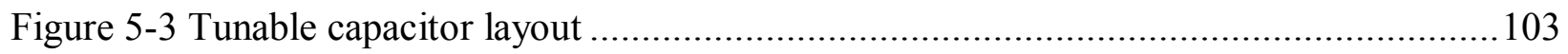

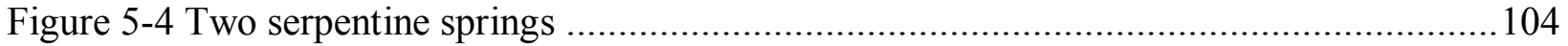

Figure 5-5 Simulated deformation (displacements) of Curve-up beam C1-C10 .................... 106

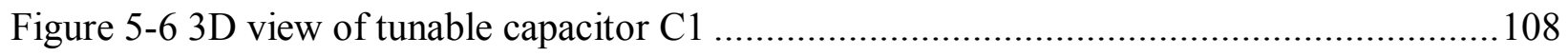

Figure 5-7 Deformations of capacitors (a) C, (b) C2 and (c) C7 ..................................... 110

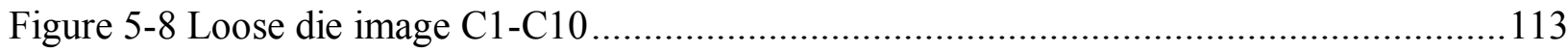




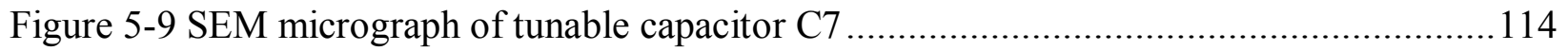

Figure 5-10 Capacitor C1 (a) surface map and (b) profile plot (c) curve-up beam plot ............115

Figure 5-11 Capacitor C5 (a) surface map and (b) profile plot (c) curve-up beam plot ............ 116

Figure 5-12 Capacitor C7 (a) surface map and (b) profile plot (c) curve-up beam plot ............ 117

Figure 5-13 Simulated and measured $H_{\max }$ values ......................................................... 119

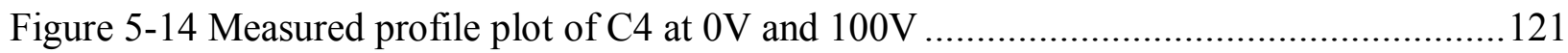

Figure 5-15 Measures profiles of C7 (a) 0V, (b) 100V, (c) 150V and (d) 162.5V................. 124

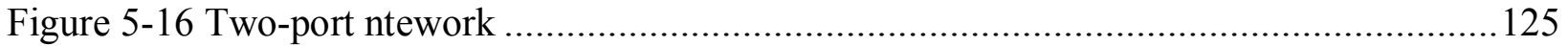

Figure 5-17 Microwave lumped model representation of capacitor .................................... 126

Figure 5-18 RF test setup at ARFSL (courtesy of CMC and UoM) ................................... 127

Figure 5-19 Measured S11 parameters of C1-C10 at 0V and 100V ................................... 129

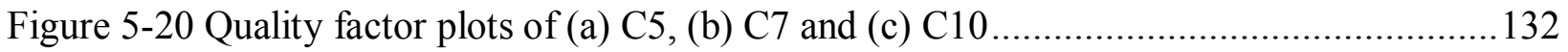

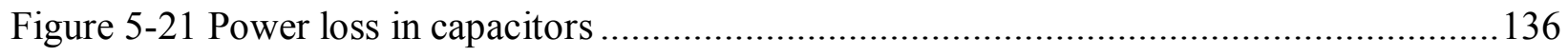

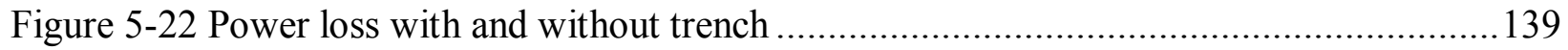

Figure 6-1 Finger tip bending vs. length of fingers ...................................................... 143

Figure 6-2 Residual stress gradient bent fingers...................................................... 144

Figure 6-3 Moving fingers and fixed fingers of the capacitor (a) and distribution of electrostatic

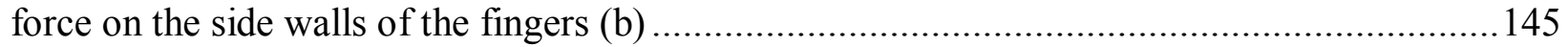

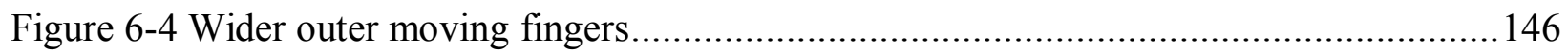

Figure 6-5 Schematic representation of optimized tunable capacitor (a) 3D view and (b) top view

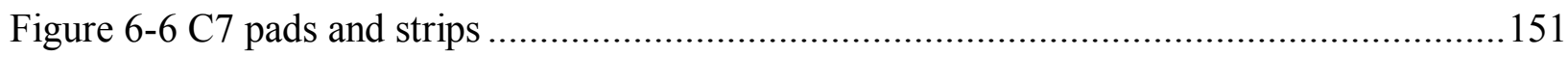

Figure 6-7 Capacitor with reduces pad, strips and anchor sizes (a) 3D view and (b) top view .. 152

Figure 6-8 Optimized capacitor (a) trench and (b) 3D view with trench ............................. 154

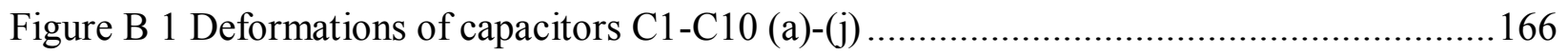

Figure C 1 Capacitor C2 (a) surface map and (b) profile plot (c) curve-up beam plot.............. 167

Figure C 2 Capacitor C3 (a) surface map and (b) profile plot (c) curve-up beam plot.............. 168

Figure C 3 Capacitor C4 (a) surface map and (b) profile plot (c) curve-up beam plot.............. 169

Figure C 4 Capacitor C6 (a) surface map and (b) profile plot (c) curve-up beam plot..............170 
Figure C 5 Capacitor C8 (a) surface map and (b) profile plot (c) curve-up beam plot..............171

Figure C 6 Capacitor C9 (a) surface map and (b) profile plot (c) curve-up beam plot.............. 172

Figure C 7 Capacitor C10 (a) surface map and (b) profile plot (c) curve-up beam plot............ 173

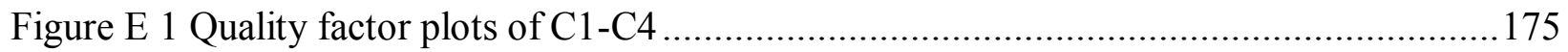

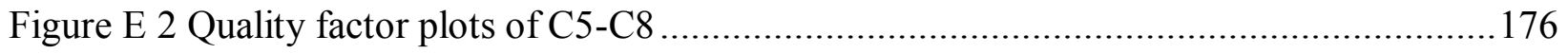

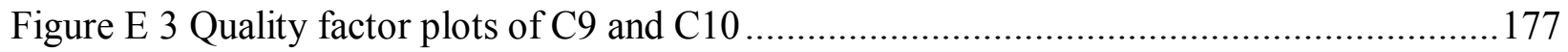

Figure F 1 D view of repulsive actuator driven tunable capacitor.................................... 178

Figure F 2 Capacitor actuator (a) surface map, (b) profile plot (c) 3D model and (d) SEM image

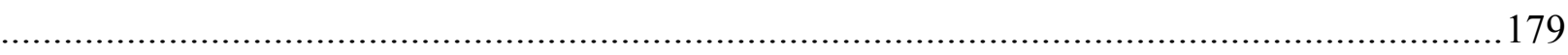

Figure F 3 Actuator moving finger (a) surface map, (b) 3D model and (c) profile plot............ 180 
Appendix A Equations of curvature of fitted curve-up beams-Matlab .................................. 162

Appendix B Deformations of curve up beams C2, C3, C4, C6, C8, C9, C10.......................163

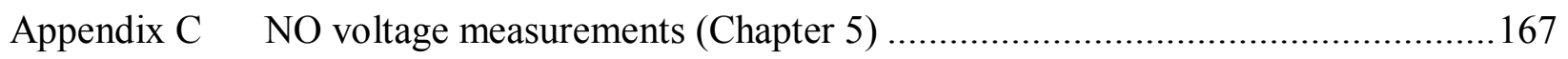

Appendix D $\quad$ Matlab code to calculate capacitance and quality factor ...................................174

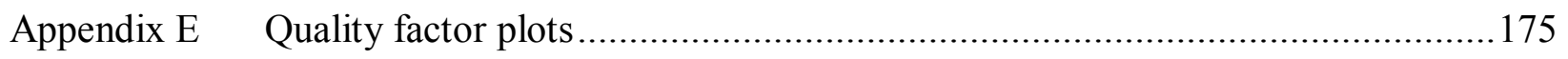

Appendix F Gap increasing tunable capacitor using gold fabrication process ......................178 


\section{Introduction}

\subsection{Motivation}

Tuning components have been integral parts of electronic circuits such as filters, impedance matching networks and oscillators used extensively in communication circuits. Most electronic communication circuits use tuning components such as varactor diodes and field-effect-transistor (FET) switches. These components have problems that degrade their performance at high microwave frequencies. One of the problems is very low capacitance tuning ratio at high frequency. The tunability of varactor diodes increases when increasing the reverse biasing voltage. The commonly used varactors are PN junction diodes 1, [2]. The width of the depletion region of positive-negative ( $\mathrm{PN})$ junction diodes increases with an increasing bias voltage. Hence the static charge accumulation decreases and leads to a decrease of the junction capacitance. To obtain a large capacitance tuning ratio, the depletion region needs to be large [3]. The increase in the gap between the PN junctions leads to a non-linear capacitance-voltage relation which is not desirable at high radio frequencies. At high bias voltages the series resistance of the PN junction increases. The increase in the series resistance causes high power loss and thus, results in low quality factors. Low quality factors may be associated with the resistivity of the silicon which is a function of the doping profile. The loss in the quality factor may be compensated by increasing the doping level. A tunneling effect can also occur with increased doping level [2] which further reduces the tuning ratio.

Tunable capacitors with high quality factors and high tuning ratios are desirable for applications at higher microwave frequencies. High quality factors can be achieved by using metal-insulatormetal (MIM) capacitors with low dielectric constants and low resistivity contact metals. The disadvantage of such capacitors is that they provide constant capacitance over all frequencies, i.e. capacitance tuning ratio of 1 which is non-tunable.

Semiconductor-switched capacitors are also tunable capacitors in current communication circuits. The switched capacitors consist of switched fixed/tunable capacitors [3-11]. The switches include positive-intrinsic-negative (PIN) diodes and complementary metal-oxide semiconductor (CMOS) diodes and high electron-mobility transistors (HEMT) such as pseudo

HEMT (pHEMT) switches. The PIN diodes have high resistance and hence power losses are very high. Thus, these devices are less useful for higher frequency applications and for wideband 
wireless applications [3-5]. The CMOS switches provide minimum capacitance at off-state and the quality factor is realized when the switch is on. The disadvantage in this case is still a very low Q factor when the switch is on. Even though it is possible to obtain a high Q by using Nchannel MOSFET switches [11], high capacitance ratios become challenging. Non-linearity is also an inherent problem of semiconductor based tunable components $[2-4,8,11]$ and is a challenge for next wireless applications. Therefore, tunable devices with low loss and high capacitance ratios are important for higher radio frequency applications.

Recent studies showed that MEMS technology has better potential to be used as tunable components for high frequency applications by offering better performance such as tuning ratios, quality factors and linearity $[4,5,12-14]$.

\subsection{What are MEMS devices?}

MEMS devices are miniature components or groups of such components with micro and submicron dimensions that combine electro-mechanical properties and are fabricated with standard IC fabrication processes. RF MEMS devices use electric or magnetic forces for actuation. The term actuation is used to describe the movement or activation of MEMS devices. Micro switches, $\mu$-tunable capacitors, $\mu$-inductors, surface acoustic wave (SAW) resonators, MEM resonators and bulk acoustic wave (BAW) resonators are some examples of MEMS devices. The common actuation mechanisms include electrostatic, electromagnetic, electro-thermal and piezoelectric actuations. Electrostatic actuators take the highest priority in practical applications that require very low power.

\subsection{Problem description and research outcome}

Several novel design concepts have been researched to enhance the performance of tunable capacitors. However, characteristics such as low tuning ratios, high loss and RF power handling are still current issues.

Conventional parallel plate tunable capacitors have a tuning ratio of $150 \%$ due to the pull -in instability caused by the electrostatic force of attraction. The electrostatic attraction force increases abruptly once the moving plate travels beyond one-third of the initial gap. The mechanical restoring force of the moving plate may not be strong enough to return the plate back to the original position. Dielectric thin films have been used as a remedy to electrically isolate the moving plate from the fixed plate and prevent the pull-in. However, the dielectric strength of 
the films does not withstand the high actuation voltages used to drive the capacitors. The power handling capability is lowered. Parallel plate tunable capacitors have two extreme values of the capacitance: the up-state and the down-state capacitance. The down state capacitance gives minimum capacitance whereas the down-state capacitance provides the maximum capacitance. The up-state capacitance yields a relatively higher quality factor than the down-state. Even higher down state capacitance can be achieved by the thin dielectric films on the fixed electrode of MEMS tunable capacitor and increases the tuning ratio. However, the quality factor corresponding to the down state capacitance is low since capacitance and quality factor are inversely related. The resistance between the plates will also increase at down state. Another disadvantage of the thin dielectric films is charge storing capability. This effect minimizes the gap between the plates of the capacitor at off-state which impacts the repeatability of the tuning ratio.

The objective of this thesis is to develop tunable capacitors that can have large tuning ratios with a high $\mathrm{Q}$ factor, linearity and high power handling capacity. The research is focused at developing designs of high quality factor RF MEMS tunable capacitors using surface micro fabrication processes. The designs will be optimized for a high tuning ratio.

\subsection{Thesis objectives}

The objectives of this thesis:

a) Design and analysis of gap increasing parallel plate tunable RF MEMS capacitors using repulsive electrostatic force actuators.

b) The design of novel actuators with moving and fixed structures from a single metal layer of Metal MUMPs process using the residual stress gradient.

c) Characterization of the actuators and tunable capacitor prototypes. The characterization includes electromechanical tests and measurements of the scattering parameters of the tunable capacitors. From the S-parameters, the impedance is extracted to determine the quality factors and the tuning ratios. The parasitic capacitances and power losses are analyzed from the S-parameters.

d) Further optimization of tunable capacitors for higher performance in terms of tuning ratios. 


\subsection{Thesis organization}

The thesis is organized as follows:

Chapter 1 summarized the motivation and highlighted the existing limitations on semiconductor based tuning components. The potential application of MEMS technology and the thesis objective are briefly mentioned.

Chapter 2 presents relevant literature reviews on tunable capacitors. The methods, fabrication processes and the performance are summarized.

In Chapter 3, repulsive electrostatic force driven (out-of-plane) RF MEMS tunable capacitors are presented. The tunable capacitors are designed and fabricated using the Poly-MUMPs micro fabrication process. Simulation results are analyzed and conclusions are drawn. Equations are derived for the capacitance between the parallel electrodes.

Chapter 4 describes the design and tests of novel vertical comb-drive actuators developed using the residual stress gradient of the nickel layer of the Metal MUMPs fabrication process. Simulations pertaining to the designs are presented in detail. Analyses of simulated results are also presented. Experimental test results of the fabricated prototypes of the actuators are included.

Chapter 5 discusses the design, fabrication and test of novel high quality factor tunable capacitors based on the actuators developed in Chapter 5 using the Metal MUMPs fabrication process. Prototypes are fabricated and experimental measurements are studied to verify the simulated performance parameters of the capacitors. The CoventorWare finite-element-analysis software package is used to design and analyze the tunable capacitors.

Chapter 6 comes up with optimization considerations of designs presented in Chapter 5. This chapter exclusively deals with increasing the tuning ratios.

Chapter 7 summarizes important outcomes of the thesis work and recommends future work. 


\section{Literature review}

This chapter presents tunable components made of semiconductors and MEMS fabrication processes. The fabrication processes of MEMS capacitors with performance parameters such as tuning ratio, quality factors and power handling are presented. The actuation mechanisms by which MEMS devices operate are briefly discussed.

\subsection{Semiconductor tunable varactors}

Semiconductor material based tunable capacitors consist of diode-varactors, Schottky diodes, positive-intrinsic-negative (PIN) diodes, tunable field-effect-transistor (FETs). The quality factors, tuning ratios of tunable filters and impedance matching networks using such semiconductor components falls with increasing bias voltages and frequency at higher RF ranges.

\subsubsection{PIN diodes}

Positive-intrinsic-negative (PIN) [15-16] diodes are used widely in microwave devices as tunable capacitors and switches. The PIN tunable capacitors and switches have tuning ratios greater than 3 [5]. However, as the tuning voltage increases the series resistance across the PN junction increases and reduces the total quality factor of the circuit. The increase in the voltage brings down the linearity of the PIN varactors. PIN varactors also require high current for operation. Figure 2-1 shows the typical PIN diodes and its layers.
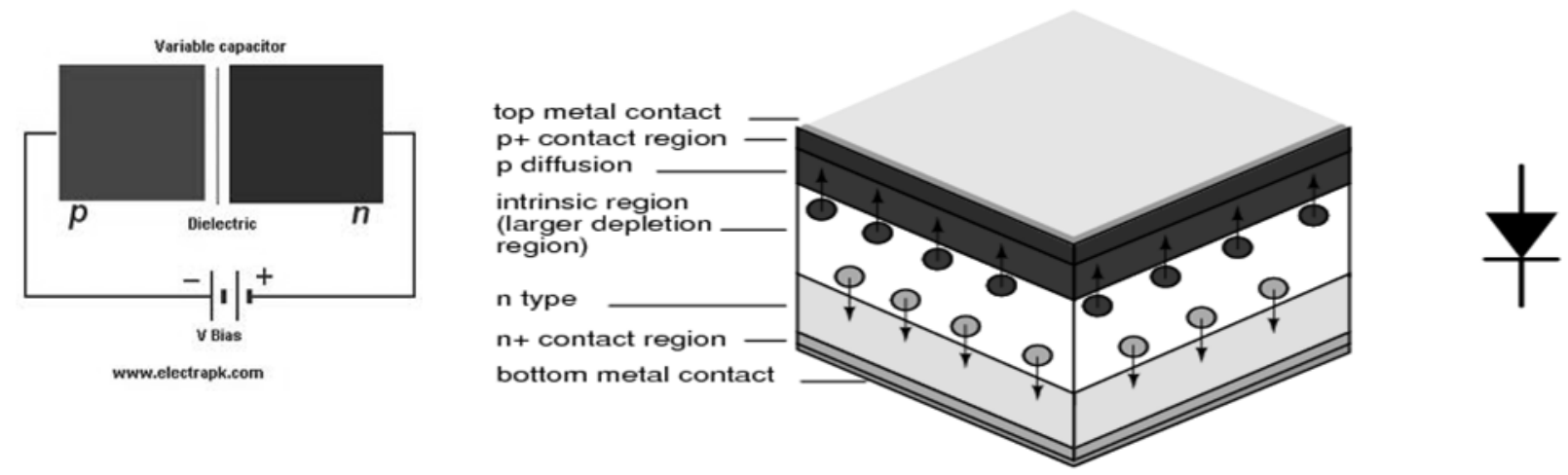

Figure 2-1 Circuit representation and layers of PIN diodes [15]

Schottky diodes are one of the favorite tuners of today's tunable filters and tunable impedance matching networks due to their good linearity $[16,17]$ though they suffer from high harmonic and sub-harmonic distortion. The Schottky-diode (Figure 2-2) capacitors use reverse biased 
diodes as tuning elements. These tunable diodes have smaller size and very fast tuning speed. However, they suffer from low power handling, non-linearity and low Q factor (of 30-100) that limits their use over narrowband microwave frequency. Due to the high loss at higher frequencies, semiconductor varactors are rarely used above $10 \mathrm{GHz}$.
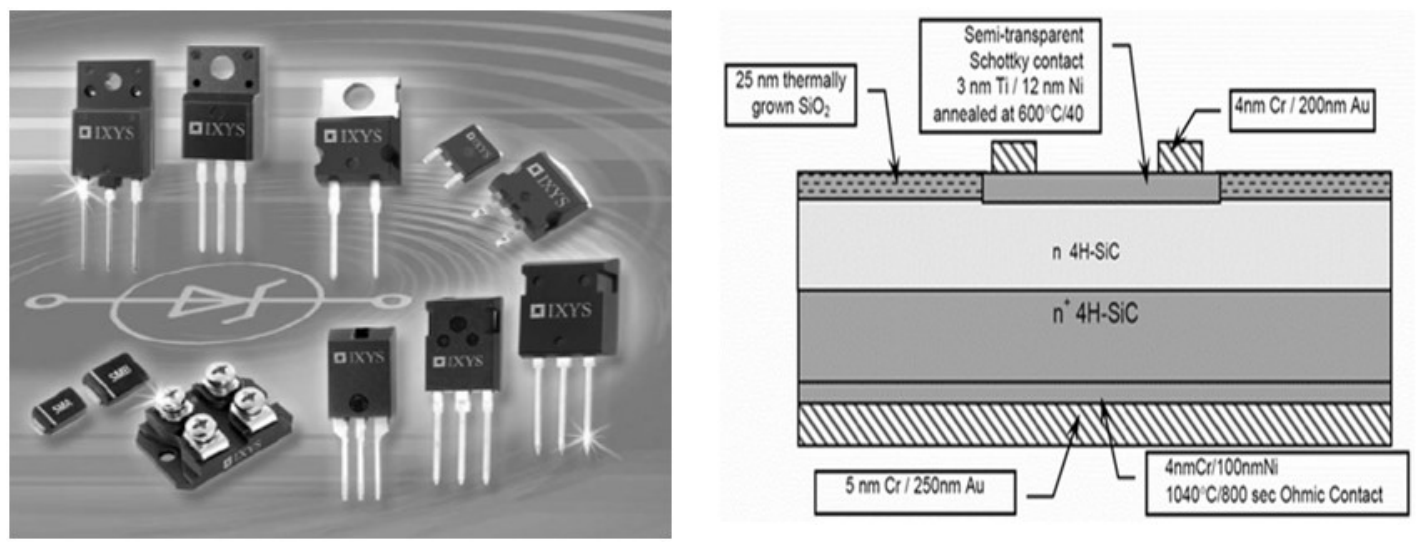

Figure 2-2 Schottky diodes and a schematic showing their construction

\subsubsection{Ferrimagnetic Tunable Filters}

Ferrimagnetic resonators, such as yttrium-iron-garnet (YIG) crystal are being used as tunable devices. YIG is actually a synthetic form of garnet. It has interesting magnetic properties that allow it to perform as a tunable microwave filter.

YIG tunable filters find wide applications in measurement instruments and base stations for cellular networks. The resonant frequency of a YIG resonator can be tuned higher by increasing the external magnetic field. YIG tunable filters offer multi-octave tuning range $(2-18 \mathrm{GHz})$ and very high quality factor $(\sim 10,000)$. One disadvantage of the YIG crystal based filters is their large power consumption of about $0.75-4.5 \mathrm{~W}$ to generate an external magnetic field and to maintain a constant temperature. It is therefore very difficult to integrate YIG filters in portable wireless devices where battery life is a critical issue. There is a minimum tuned frequency when the external magnetic field equals to the demagnetizing field strength. Although YIGs provide a very high quality factor, they are known for low tuning speed; and heavy weight that causes difficulty to integrate in planar fashion [17-19] for mobile devices.

In contrast, micro-electromechanical systems (MEMS) can be used to get tunable capacitors by applying a DC voltage. When a MEMS device is used at microwave radio frequency, it is referred to as radio frequency (RF) MEMS device. RF MEMS switches with high capacitance 
tuning ratios have been in use at millimeter wave frequency with high $\mathrm{Q}$ factors [21]. Tunable capacitors fabricated using MEMS fabrication processes offer better performance because of their desirable characteristics compared to their semiconductor counterparts. The first exciting feature of MEMS tunable capacitors is the insensitivity to low distortion since MEMS tunable capacitors are not susceptible to harmonics. Second, MEMS tunable capacitors have better linearity at high frequencies.

Tunable capacitors are building components for radio frequency devices such as filters, resonators, oscillators and tunable antennas. They have great potential to be used for selfadaptive impedance matching networks in cell phones, wireless devices and cognitive radios.

\subsection{RF MEMS tunable capacitors review}

RF MEMS switches and tunable capacitors have been developed for switching/filtering circuits that could be used in microwave and millimeter wave frequencies and substantial effort has been put to the development of phase shifters, tunable filters and matching networks. Several research studies are being carried out since 1996 which was the year the first micro-machined tunable capacitor was reported.

\subsubsection{Parallel plate electrostatic actuator tunable capacitors}

The simplest tunable capacitors consist of two parallel plate separated by a distance $z$ one being suspended while the other is fixed. Figure 2-4 shows the schematic diagram a parallel plate electrostatic actuator tunable capacitor. When a voltage is applied across the plates, electrostatic force builds up and the suspended plate moves towards the fixed plate. As the voltage increases the gap between the plates gets narrower.

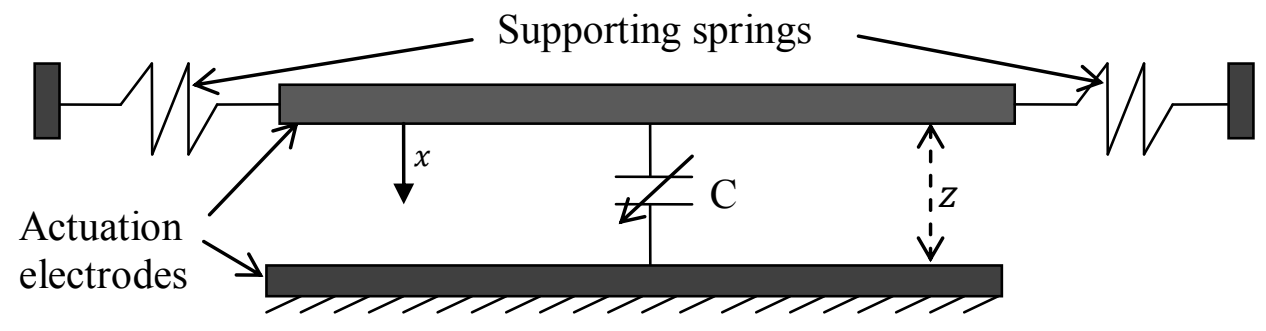

Figure 2-3 Traditional parallel plate electrostatic actuator tunable capacitor [20] The magnitude of the electrostatic force is given by [22] 


$$
F_{e}=\frac{1}{2} \frac{C V^{2}}{z}
$$

where $C$ is the parallel plate capacitance, $C=\varepsilon A / z, A$ is the parallel plate area, $\varepsilon$ is the absolute permittivity of the medium between the parallel plates and $z$ is the gap between the plates. As the actuation voltage increases the gap between the plates decrease. The restoring force generated by the supporting springs is given as $F_{r}=K_{s} x, x$ is the displacement the moving plate travels at a given actuation voltage $\mathrm{V}$. At equilibrium, the electrostatic force and the spring force are equal. Capacitors realized with such designs achieved 150\% tuning ratio. The plates of the capacitors suffer from pull-in effects after the moving plate travels one-third of the original gap $(x=z / 3)$.

\subsubsection{Parallel plate tunable capacitors with different gaps}

Zou et al. [24] developed a dual gap parallel plate tunable capacitor [24] to increase the stroke and the tuning ratio. In this design, a tunable capacitor which consists of two parallel plate actuators and one parallel plate capacitor were developed. The gap between the parallel plates of the actuator is greater than gap of the capacitor electrodes. The maximum theoretical tuning ratio (TR) of this capacitor is given by

$$
T R=\frac{z_{1}}{3 z_{2}-z_{1}}
$$

The measured capacitance is $32 \mathrm{fF}$ at $0 \mathrm{~V}$ with a tuning ratio of $69.8 \%$.

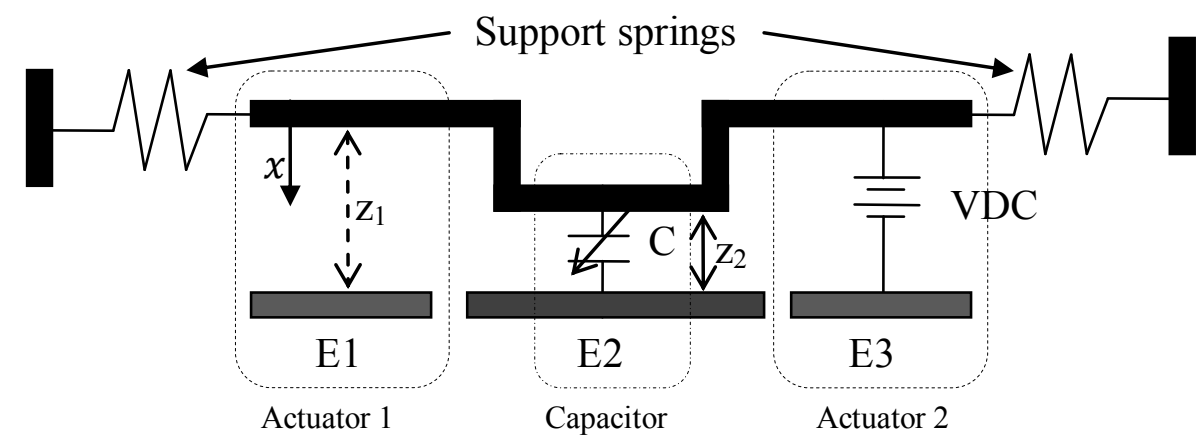

Figure 2-4 Dual gap tunable capacitor [24] 


\subsubsection{Zipper tunable capacitors}

A zipping type cantilever based tunable capacitors [23] are another class of capacitor by which tuning is achieved by a zipping action. Zipper capacitors, Figure 2-5, mainly consist of two parallel plates; cantilever beam and a fixed plate (with dielectric on top) printed on a high dielectric substrate. When the actuation voltage increases the gap, $z$, between the cantilever beam and the fixed electrode decreases to increase the capacitance.

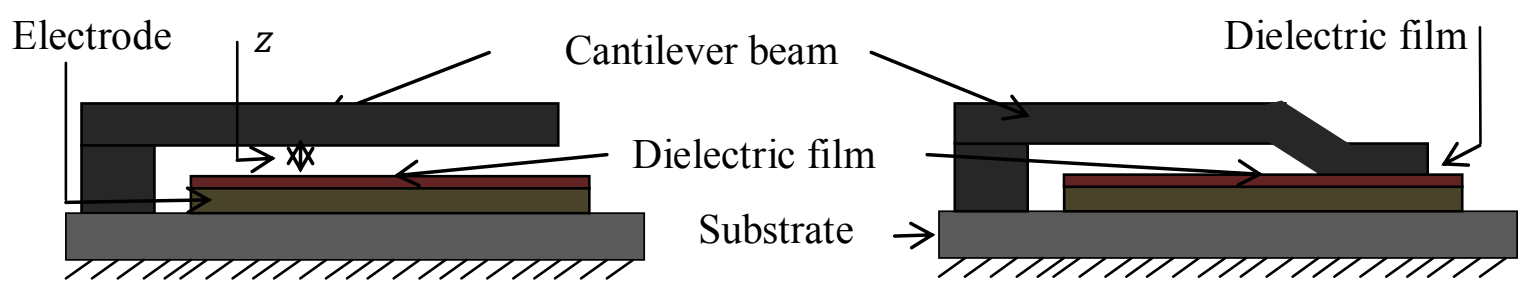

Figure 2-5 Cantilever beam zipper

\subsubsection{MEMS bridges as switchable capacitor}

Switchable MEMS bridges are also used to design tunable capacitors [25]. The designs here consist of suspended or fixed-fixed MEMS membrane on a coplanar waveguide micro strip lines (Figure 2-6). Raytheon Systems Company developed switched MEMS tunable capacitor banks for $0.1 \mathrm{GHz}$ to $6 \mathrm{GHz}$ applications [25] using MEMS bridges. The array of such individual components in a shunt or series connection was used to build multi-bit capacitor banks for 1$10 \mathrm{GHz}$ operation.
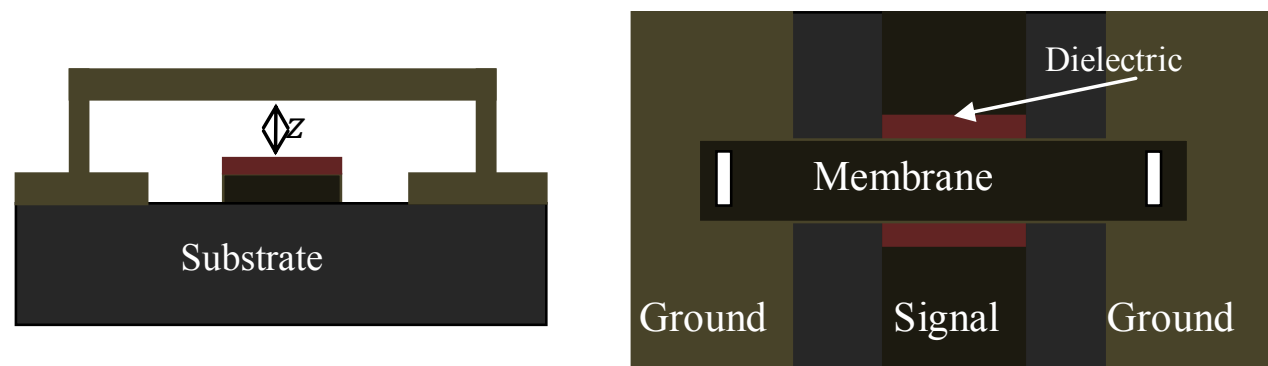

Figure 2-6 RF MEMS switchable capacitor [25] cross section view (left) and top view (right) 


\subsubsection{Flip-chipped digitally controlled cascaded capacitors}

Hoivik et al. developed MEMS varactors by using 30 different cascaded capacitors of equal area $30 \mu \mathrm{m} \times 100 \mu \mathrm{m}$ next to each other actuated by a uniform electrostatic force. The variable MEMS tunable capacitor (Figure 2-7) is fabricated using Cronos MUMPs process, and is transferred to an alumina substrate using a flip chip assembly process. Each plate of the capacitor is connected to a bonding pad by beams of variable spring constants that deform the plates to stick down in cascaded manner to form metal-air-metal capacitors [26, 27]. The capacitor has achieved tuning ratios of $400 \%$ with measured quality factors of 140 at $750 \mathrm{MHz}$.
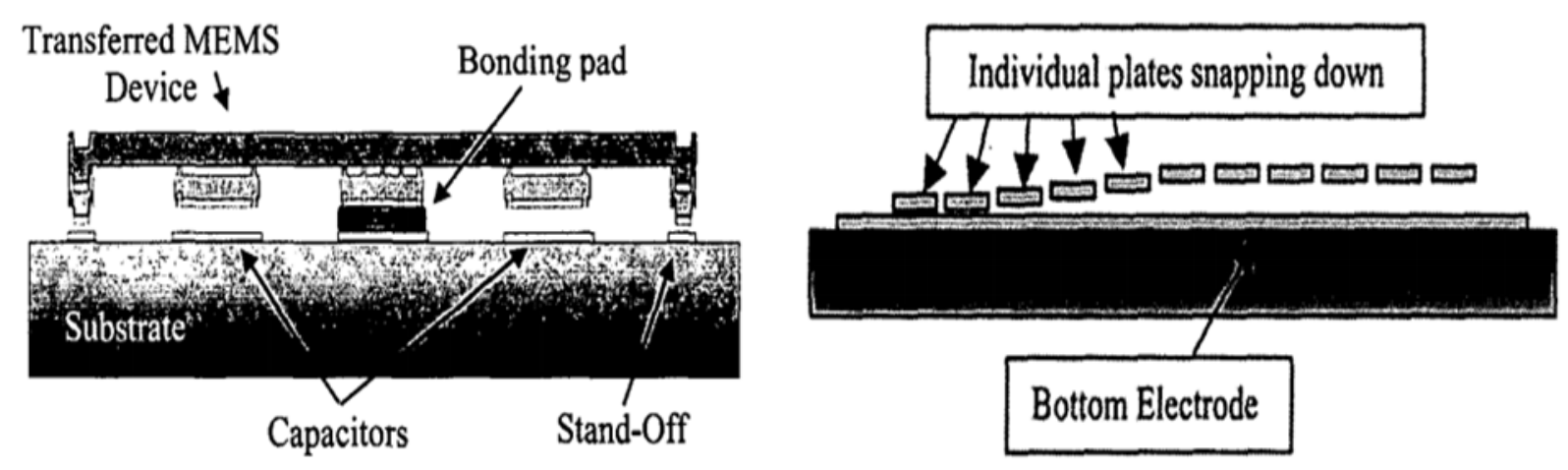

Figure 2-7 Digitally controllable capacitors developed using flip chip process [26]

The fabrication process consists of six layers: three poly-silicon layers, two oxide layers, one gold layer and alumina substrate. The top layer is made of $2 \mu \mathrm{m}$ thick first poly-silicon, $0.75 \mu \mathrm{m}$ oxide, $1.5 \mu \mathrm{m}$ thick second poly-silicon and $0.5 \mu \mathrm{m}$ gold. The purpose of the gold layer is to increase the electrical conductivity, signal probing and bonding pads. The authors mentioned that the silicon substrate is etched away after the flip chip step because it was found conductive due to the doping. The advantage obtained by removing the silicon had brought better quality factor [27]. The fabricated tunable capacitor of arrays of 30 occupied a total area of $0.5 \mathrm{~mm} \times 1.0 \mathrm{~mm}$. The material used to print the bonding pads on the alumina substrate is indium and deposited with lift-off process. Finally, the two substrates are bonded together with a thermo compression technique. The sacrificial layer is removed with dilute hydrofluoric (HF) acid solution to open an air gap of $2 \mu \mathrm{m}$. 


\subsubsection{Comb actuator type capacitors}

Comb actuators are being used to drive tunable capacitors [30]. Comb actuators have two sets of finger like electrodes one moving the other fixed as shown in Figure 2-8 and 2.9. Initially the moving fingers and the fixed fingers are engaged in some overlap distance z. The tunable capacitance is the capacitance between the between the side walls of the fingers and it is proportional to the overlap area. Tunable capacitors with comb actuators have high stroke without pull-in effect however, they provide low quality factors at high frequency. The process used to realize comb capacitors is bulk micromachining of SOI which is expensive and complex process.

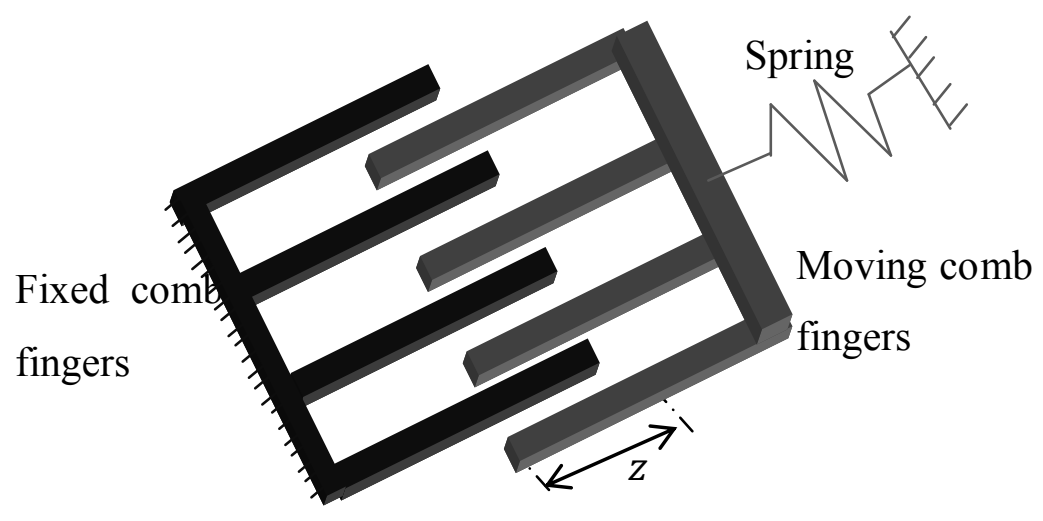

Figure 2-8 Comb drive actuator
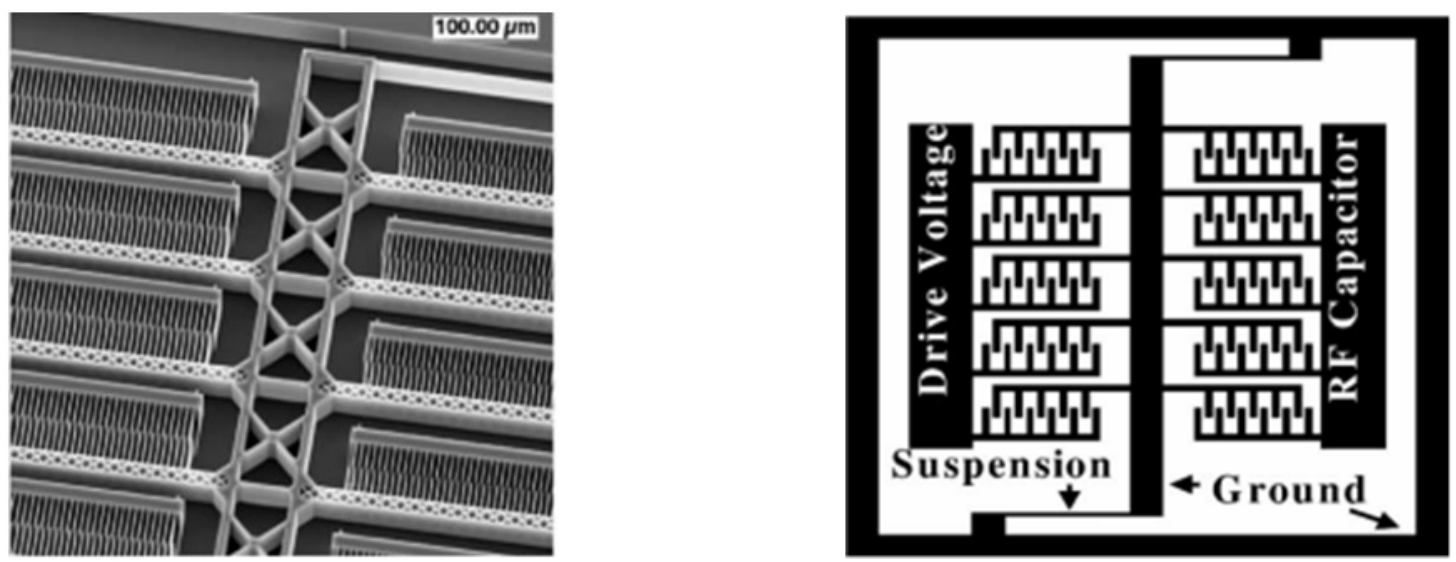

Figure 2-9 SEM image of $40 \mu \mathrm{m}$ thick tunable capacitor with comb drive actuator (left) and the schematic diagram representation (right) (R. L. Borwick et al. [30, 31]) 


\subsubsection{Tunable capacitors summary}

Table 2-1 MEMS Tunable capacitors

\begin{tabular}{|c|c|c|c|c|c|}
\hline Capacitor & $\begin{array}{l}\text { Tuning } \\
\text { ratio }(\%)\end{array}$ & Quality factor & $\begin{array}{l}\text { Linearity } \\
(\%)\end{array}$ & $\begin{array}{l}\text { Power } \\
\text { handling } \\
(\mathrm{dBm})\end{array}$ & Material and processes \\
\hline $\begin{array}{l}\text { Parallel plate actuator } \\
\text { Young and Boser [20] }\end{array}$ & 15 & 60 at $1 \mathrm{GHz}$ & NA & NA & Aluminum \\
\hline $\begin{array}{l}\text { Dec and Suyama [21] 2-palte and } \\
\text { 3-plate capacitor }\end{array}$ & $\begin{array}{l}151 \text { and } \\
187\end{array}$ & 20 and 16 at $1 \mathrm{GHz}$ & NA & NA & PolyMUMPs \\
\hline Zou [24] three electrodes & 69.8 & NA & NA & NA & Pyrex glass, perm alloy \\
\hline $\begin{array}{l}\text { S. Jung et al Cantilever beam } \\
\text { tunable capacitor }[22,23]\end{array}$ & 116 to 118 & NA & NA & NA & PolyMUMPs/glass quartz/gold \\
\hline $\begin{array}{l}\text { Rockwell science [22] inter- } \\
\text { digital capacitor }\end{array}$ & 410 & $\begin{array}{l}160 \text { to } 40 \text { at } 0.4 \text { to } \\
1.6 \mathrm{GHz}\end{array}$ & NA & NA & SOI glass bonding \\
\hline $\begin{array}{l}\text { Ryethon systems company [25] } \\
\text { switched capacitor }\end{array}$ & NA & $\begin{array}{l}40 \text { to } 80 \text { over } 1- \\
10 \mathrm{GHz}\end{array}$ & NA & NA & $\mathrm{Al} /$ silicon nitride \\
\hline $\begin{array}{l}\text { Hoivok et al. [26] } 30 \text { cascaded } \\
\text { capacitors }\end{array}$ & 400 & 140 at $750 \mathrm{MHz}$ & NA & NA & $\begin{array}{l}\text { Cronos MUMPs to alumina substrate using } \\
\text { flip chip assembly process bonding }\end{array}$ \\
\hline $\begin{array}{l}\text { Differential multi-finger capacitor } \\
\text { [27] }\end{array}$ & 470 & 200 at $1 \mathrm{GHz}$ & NA & NA & PolyMUMPs \\
\hline $\begin{array}{l}\text { Differential Multi-Finger MEMS } \\
\text { Tunable } \\
\text { Capacitors [28] }\end{array}$ & $1.48-3.53$ & 17 at $1.5 \mathrm{GHz}$ & NA & NA & PolyMUMPs process \\
\hline $\begin{array}{l}\text { Comb finger capacitors } 608 \\
\text { fingers [29] }\end{array}$ & 10 & 4 at $2 \mathrm{GHz}$ & NA & NA & $\begin{array}{l}\text { Single crystal silicon/Pyrex glass bonding } \\
\text { process/copper }\end{array}$ \\
\hline $\begin{array}{l}\text { R. L. Borwick [30, 31] similar to } \\
\text { [30] }\end{array}$ & 840 & 100 for 0.2 to $0.4 \mathrm{GHz}$ & & & $\begin{array}{l}\text { Silicon between aluminum coats/SOI epoxy } \\
\text { bonded to glass wafer }\end{array}$ \\
\hline $\begin{array}{l}\text { A High-Q Tunable } \\
\text { Micromechanical Capacitor With } \\
\text { Movable Dielectric [32] }\end{array}$ & 7.7 and 40 & 291 and 248 & NA & NA & NA \\
\hline $\begin{array}{l}\text { High tuning range AlSi RF } \\
\text { MEMS capacitors [33] }\end{array}$ & $100 \%$ & NA & NA & NA & Custom design \\
\hline Dual gap relay type [34] & 1200 & 150 at $0.5 \mathrm{GHz}$ & NA & NA & PASSI process silicon/ aluminum alloy, \\
\hline
\end{tabular}




\begin{tabular}{|c|c|c|c|c|c|}
\hline & & & & & three metal layers and two dielectric layers \\
\hline Nitride loaded capacitor [35] & 280 & 13 at $1 \mathrm{GHz}$ & NA & NA & MetalMUMPs \\
\hline $\begin{array}{l}\text { Switched capacitor with landing } \\
\text { pads [36] }\end{array}$ & $150-200$ & 225 at $\mathrm{X}$ and $\mathrm{Ku}$ & NA & $1 \mathrm{~W}$ & Glass, Ti/Au, Ti/Au/Ti, gold (5um) \\
\hline $0.35 \mathrm{~mm}$ CMOS process [37] & 85 & 40 at $1 \mathrm{MHz}$ & NA & NA & \\
\hline $\begin{array}{l}\text { Bulk micro machined capacitors } \\
\text { for VCOs [38] three designs }\end{array}$ & $\begin{array}{l}5.88,6.98 \\
10.53\end{array}$ & $\begin{array}{l}136,167,156 \\
\text { respectively for } \\
\text { unknown frequency }\end{array}$ & NA & NA & $\begin{array}{l}\text { Pyrex glass trenched silicon, } \mathrm{Cr} / \mathrm{pt} \text {, } \\
\text { aluminum electrodes }\end{array}$ \\
\hline $\begin{array}{l}\text { Floating electrode capacitor, } \\
\text { Yoon [39] two designs }\end{array}$ & 29,41 & $\begin{array}{l}39.8 \text { at } 1 \mathrm{GHz} \text { and } 34.9 \\
\text { at } 5 \mathrm{GHz} \text { for four } \\
\text { capacitor arrays }\end{array}$ & NA & NA & Copper \\
\hline $\begin{array}{l}\text { Stressed Dual-gap RF MEMS } \\
\text { Varactors [40] }\end{array}$ & 621 & 50 at $30 \mathrm{GHz}$ & NA & NA & Customized process \\
\hline $\begin{array}{l}\text { Floating/movable dielectric [41] } \\
\text { similar to [39] }\end{array}$ & 367 & 56 at $1 \mathrm{GHz}$ & NA & NA & SOI MUMPs \\
\hline $\begin{array}{l}\text { RF MEMS switched three step } \\
\text { capacitor [42] }\end{array}$ & NA & $>100$ at $10 \mathrm{GHz}$ & NA & NA & ------- \\
\hline $\begin{array}{l}\text { Laterally driven tunable } \\
\text { capacitors [43] }\end{array}$ & 260 & NA & NA & NA & Double layer polysilicon and $\mathrm{SiO}_{2}$ \\
\hline $\begin{array}{l}\text { Triangular parallel plate, } \\
\text { Shavezipur [44] }\end{array}$ & 150 & NA & NA & NA & PolyMUMPs \\
\hline $\begin{array}{l}\text { Capacitor For High Q Millimeter } \\
\text { Wave Tuning [45] }\end{array}$ & 350 & NA & NA & NA & \\
\hline $\begin{array}{l}\text { Analog inter-digital segmented } \\
\text { electrodes [46] }\end{array}$ & $500-900$ & 100 at $\mathrm{X}$ and $\mathrm{C}$ bands & NA & 0.6-1.1 W & Alumina/gold/silicon nitride \\
\hline $\begin{array}{l}\text { Zipper Inter-digital segmented } \\
\text { electrodes [47] }\end{array}$ & 300 & 100 at $3 \mathrm{GHz}$ & NA & NA & Alumina/gold/silicon nitride \\
\hline Quadruple Series Capacitor [48] & 289 & 150 & NA & $+36 \mathrm{dBm}$ & Unknown \\
\hline
\end{tabular}




\subsection{MEMS Fabrication Processes}

The two commonly known MEMS fabrication processes are surface micromachining and bulk micromachining processes. In surface-micromachining processes (Figure 2-10), MEMS components are developed using succession of layer-by-layer deposition of structural materials and removal of sacrificial materials through lithography. A base material, usually high-grade (100) silicon substrate fabricated using Czochralski process [49], is a starting material for the process. On the silicon substrate, thin films of metal or dielectric structural layers are deposited with the help of defined deposition techniques.

Electrochemical deposition (electroplating) and physical vapor deposition (PVD) (e.g. evaporation, sputtering and pulsed laser deposition) are the techniques most frequently used to deposit thin metal films. However, chemical vapor deposition (CVD) [50, 51] is the preferred technique to deposit thin dielectric films, semiconductors and poly-silicon.

Bulk micromachining is the process of developing MEMS devices from bulk substrates and wafers. The bulk wafer is patterned using etching to transfer the patterns through photo-masks.
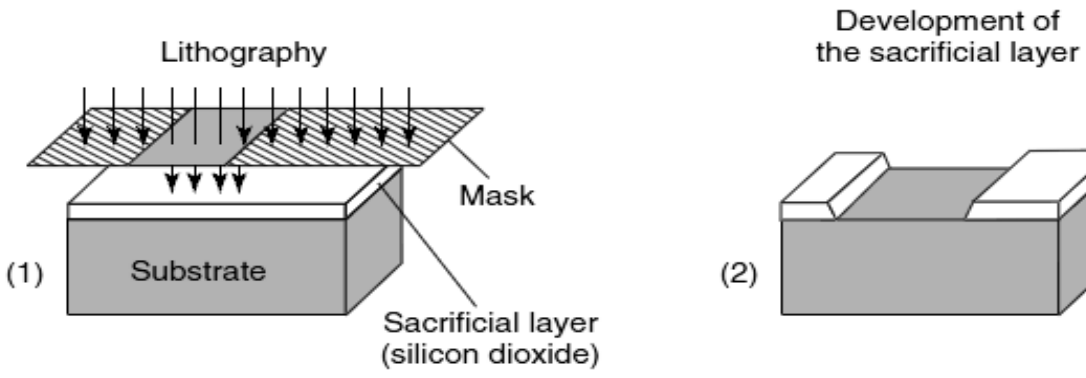

Deposition of the structural layer

(2)

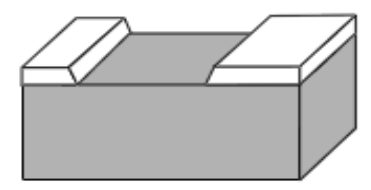

(3)
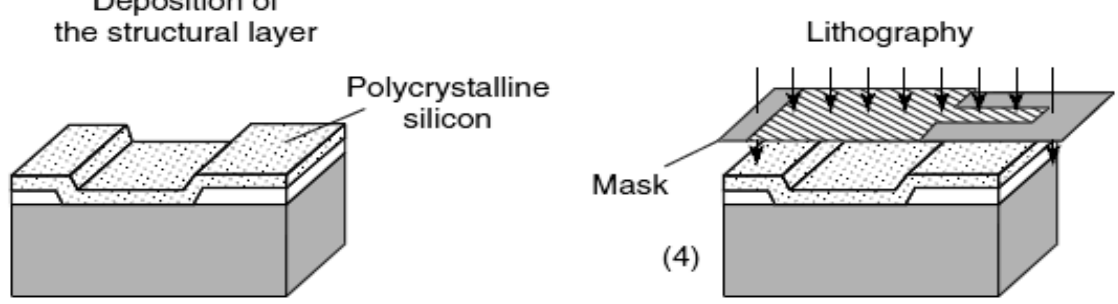

Patterning of the structural layer

(5)
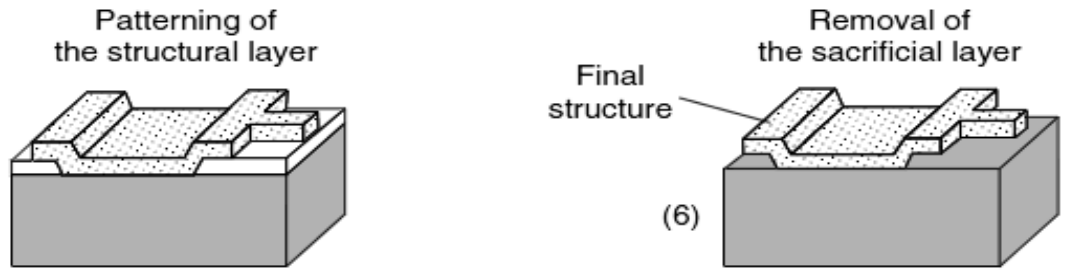

Figure 2-10 Illustration of the basic process flow in surface micromachining [50] 


\subsection{Materials for RF MEMS capacitors}

RF MEMS capacitors require materials with low loss and good conductivity [50]. MEMS materials can be classified into three categories based their function as: base materials, sacrificial materials and structural materials. MEMS fabrications start with deposition of thin films on high resistive substrate which is the base material. Common examples of elemental substrate materials include semiconductors silicon and germanium. Semiconductor compound substrates such as alumina, gallium-arsenide and indium-phosphide are also materials of choice. Nonsemiconductor low dielectric substrates quartz and sapphire found applications as a base material. Substrates with low dielectric constant and low resistivity yield better performances at microwave frequencies.

Sacrificial materials are the materials which are removed by subtractive processes to release moving parts of MEMS devices at the final stages of the fabrication process. Silicon dioxide and polymeric materials are suitable for such purposes.

The structural materials of MEMS devices are parts that remain after fabrication as a movable or fixed layers for device functioning. Metals, poly-silicon, dielectrics, thin membranes constitute of the structural materials. High performances can be achieved with high conductivity metals at microwave frequencies.

\subsection{MEMS actuators}

Micro-electromechanical components require actuation to operate. Actuation is the act of driving a device/component by an external source of energy. Devices can be actuated varies using electrical, mechanical, hydraulic or pneumatic [52] driving sources. MEMS devices are actuated by either electrostatic, electro-thermal, electromagnetic or piezo-electric/piezo-resistive [53] mechanisms.

\subsubsection{Electrostatic actuation}

Electrostatic force is a surface force suitable for micro actuation. Electrostatic forces are less used for driving devices of size in macro-scale. However, for MEMS device, which have high surface area-to-volume ratio, electrostatic actuation are attractive. Electrostatic forces are results of potential difference between two surfaces that store charges. Capacitance is the property that quantifies how much charge is stored between two parallel surfaces (e.g. conductors). It is used 
as either a sensing element or actuating element. When a voltage difference is applied across two parallel conductors separated by a dielectric medium, charges start to accumulate over the surfaces of the conductors. The capacitance between the plates can be changed by the electrostatic force which is generated by the charges stored on the plates. The capacitance of parallel electrostatic actuators is given by:

$$
C=\frac{\varepsilon A}{z} .
$$

where $A$ is the area of the parallel conductors, $\varepsilon$ is the absolute dielectric constant of the dielectric medium and $z$ is the distance between the conductors.

The electrostatic force is proportional to the capacitance, the square of the voltage and inversely proportional to the separation between the plates [54] as given by.

$$
F \propto \frac{C V^{2}}{z} .
$$

Electrostatic actuators have some important advantages over other MEMS actuators:

(1) Simplicity- the design principles are simple since parallel plate conducting structures are used for fabrication.

(2) Low power- electrostatic actuators rely on potential difference between two charged conductors. At static condition and low frequency applications, the actuators consume very low power.

(3) Fast response- good conductors provide low charging and discharging time constants. Therefore, electrostatic actuators offer fast dynamic-response.

(4) Integration- they can be easily integrated to monolithic microwave integrated circuit (IC).

Another type of electrostatic actuators are inter-digitized fingers (IDT), which have shapes like teeth of a comb, commonly known as comb-drive actuators. There are two sets of fingers (fixed and moving) on the same plane and the comb fingers are engaged with an overlapping distance $d$. If the two sets of the fingers have identical thickness $\delta$, and separated by a horizontal distance $x$ from an immediately neighboring finger, the capacitance is:

$$
C \propto \frac{\delta d}{x} .
$$




\subsubsection{Electro-thermal actuation}

Electro-thermal actuators use heat to deform or move a structure through thermal expansion or contraction [55]. Ohmic (joule) heating, conduction, convection or electromagnetic waves are conventional techniques that can raise the temperature of micro-devices [53].

The kinetic energy of atomic particles transmits temperature in a micro-device with non-zero temperature gradient. Heat can be transferred through convection, conduction and radiation. Conduction refers to the transfer of heat through a solid structure in the presence of temperature gradient. Convection is the transfer of heat from a surface to a static body of fluid. Radiation deals with the loss or gain of heat through radiation in vacuum or air.

In micro-machined devices, conduction is the most widely used method of thermal actuation. Thermal resistance is a quantity that describes the transfer of heat inside solid conductor between two points. Higher thermal resistance provides higher thermal isolation with smaller heat transfer rate under a given temperature.

Current flowing through a metallic conductor of length $l$ and thermal conductivity $k$ has heating effect. For a conductor of cross sectional area $A$ and resistivity $\rho$, the heat $Q$ flowing through it at change of temperature $\Delta T$ is:

$$
Q=-k A \frac{\Delta T}{l}
$$

The thermal resistance is:

$$
R=\left|\frac{\Delta T}{Q}\right|=\rho \frac{A}{l}
$$

Bimetallic thermal effect is thermal actuation technique used for many applications. Thermal bimetallic principle consists of two dissimilar materials with different thermal expansion coefficients. When a heat source is connected across the terminals of the two materials, the conjugate material starts to bend towards the direction of the material with the lower thermal expansion.

Thermal actuator can also be single material type. Such thermal actuators have been reported in $[56,57]$. Chevron (bent) and pseudo-morph electro-thermal actuators are the long established actuators. When a potential difference is applied to the terminals of the pseudo-morph type actuators current starts flowing, and increases the temperature of the thinner arm, known as hot arm. The second arm of the actuator has relatively lower temperature (known as cold arm). 
Because of the temperature difference, the hot arm causes the lateral deflection of the actuator that is determined as displacement.

The chevron actuators have a bent or v-type design with symmetrical configuration. When voltage is applied across ends of the actuator, the temperature increases and pushes the tip of the actuator forward.

\subsubsection{Electromagnetic actuation}

Magnetic fields have been used to produce force, displacement or torque on micro-devices. A magnetic field can induce force on current carrying conductors, coils, magnetic material, or magneto-strictive materials [53]. Ferromagnetic materials are good magnetic materials since they have high permeability.

Electromagnetic actuators were being developed in the past [58-60] with different design principles. The basic principle of operation of magnetic actuators relies on Lorentz force or reluctance actuation [61]. Magnetic actuators are reported to provide higher stroke over electrostatic and thermal actuators but at a cost of further design complexity and difficulty for integration with electronic circuits.

\subsection{Characteristics of RF MEMS tunable capacitors}

\subsubsection{Insertion loss}

In radio frequency terminology, the term insertion loss refers to the loss of signal power resulting from the insertion of a device in RF circuit or microwave transmission lines. RF MEMS tunable capacitors have very low on state impedance which results in high isolation. It is expressed in decibels $(\mathrm{dB})$.

\subsubsection{Linearity}

Linearity is defined as the response to a fast oscillating electric signal at high frequency (usually above $1 \mathrm{MHz}$ ). Because the mechanical resonant frequency of MEMS devices is less than the RF frequency there is less probability of creation of harmonics. Therefore, MEMS capacitors are linear and produce low inter-modulation products.

\subsubsection{Lower power consumption}

Electrostatic actuated MEMS devices use high DC voltage for driving, but they draw very low current. Hence, the power consumption is very low. 


\subsubsection{High isolation}

MEMS devices usually have an air gap between metal-metal and metal-dielectric contacts, which drives them for applications requiring high isolation above $40 \mathrm{GHz}$.

\subsubsection{Inter-modulation-product}

MEMS switches are linear devices, and thus have low inter-modulation products. Their performance is around $30 \mathrm{~dB}$ better than PIN or FET switches.

\subsection{Chapter summary}

The literature review explored the up-to-date semiconductor and MEMS capacitor designs, fabrication based on some performance metrics. The literature suggests that there is great interest for the development of high quality tunable capacitors for the next emerging wireless devices. Because tunability of a wireless device enables wide coverage of microwave frequency bands. It was reported that over the next five years MEMS tunable components would be used in cell phone circuits to support more than 10 channels of multi-radio services. For this purpose, devices with high tuning ratio more than 3:1 and high quality factors are in demand. It has been reported in many of the studies listed in the chapter that high quality factor is required to compensate for the losses that occur on the RF board of an electronic circuit.

Chapter 3 focuses on the design and analysis of a gap increasing tunable capacitors to achieve high tuning ratio. Parallel plate attraction electrostatic actuators suffer from pull in effects and the travel range of the plates of the capacitor is limited to one-third of the gap between the plates. The tuning ratio such capacitors is $150 \%$. A repulsive electrostatic actuator which is able to push the moving plate of a tunable capacitor upward is used to achieve large tuning ratio. Chapter 3 solely presents the design, analysis and tests of repulsive electrostatic actuator driven tunable capacitor. Hence, the advantage of the repulsive actuator is higher tuning ratio without pull-in effect.

In Chapter 4, a novel vertical comb drive actuator is developed from a single layer. The residual stress gradient deformation is used as method to create vertically separated comb structures. Simulations and the experimental results are discussed in detail. Chapter 5 discusses the design of the comb drive actuator with application to a tunable capacitor. High tuning ratio of the combdrive actuator is obtained through the optimized design in Chapter 6. 


\section{A Repulsive Electrostatic Force Driven RF MEMS Tunable Capacitor}

\subsection{Introduction}

In this chapter, a novel repulsive electrostatic force driven tunable capacitor is designed using the PolyMUMPs process [62]. The proposed tunable capacitor has three unique features compared with traditional parallel plate tunable capacitors to achieve superior performance. These features are (1) the capacitor is driven by a repulsive electrostatic force to move the plate upward, (2) no pull-in effect, (3) high tuning ratio and (4) high linearity. Two designs of the repulsive force driven tunable capacitors are presented. In Design 1, the capacitance is tuned through translating the moving plate. In Design 2, the capacitance is tuned by rotating the moving plate.

\subsubsection{Repulsion Electrostatic Force Actuation}

Repulsive electrostatic force actuators produce out of plane force directed upward to obtain a large stroke without the pull-in effect [63-65]. For a repulsive electrostatic force to occur, fingers are arranged in the manner shown in Figure 3-1 [63-65]. There are arrays of aligned fingers and unaligned fingers. The aligned fingers consist of fixed aligned fingers and moving aligned fingers. At zero volt, the moving fingers and the fixed fingers are parallel i.e. the angle between them is zero.

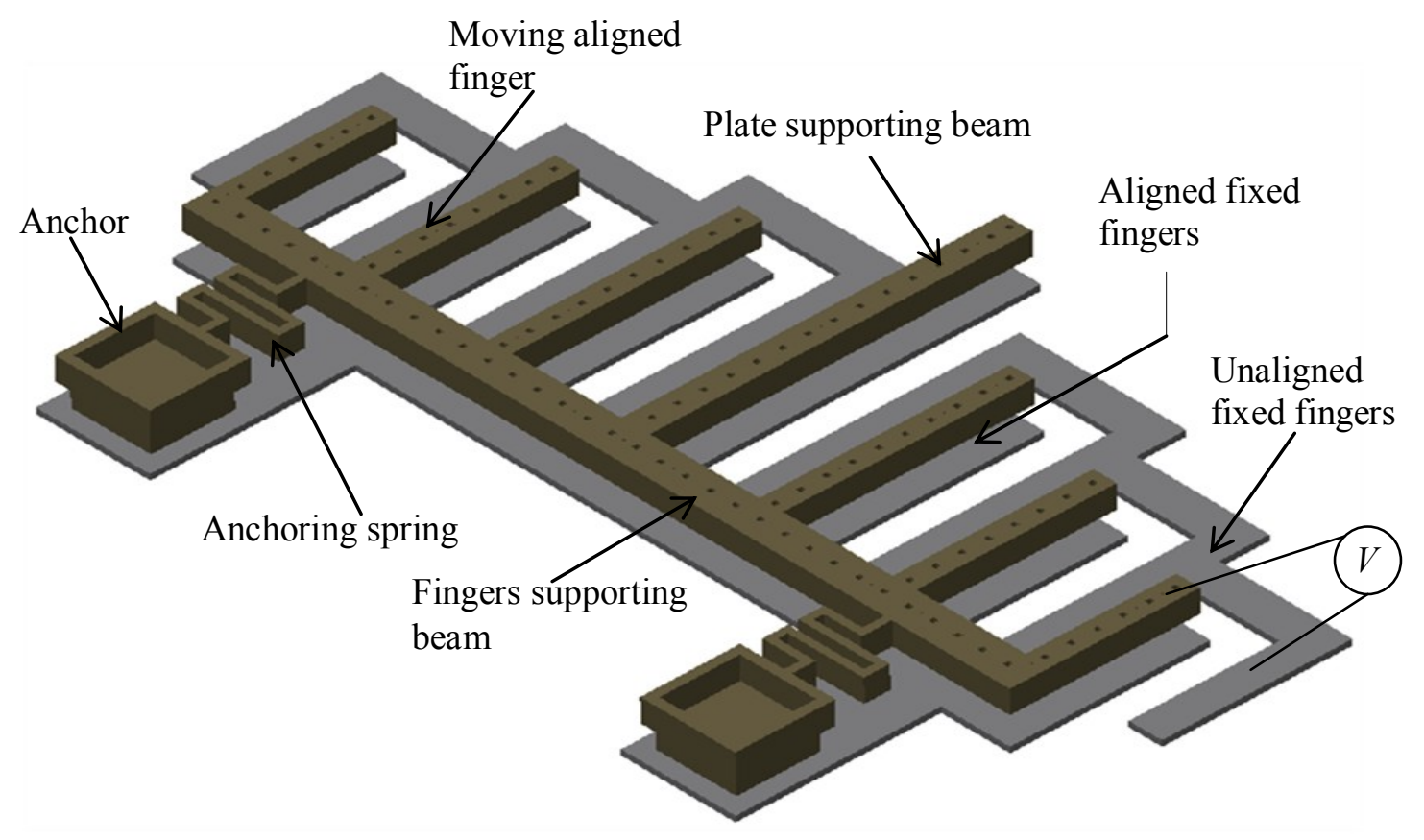

Figure 3-1 Repulsive electrostatic actuator 3D view with $V=0$ [63] 
The initial gap between the fixed and the moving fingers is $2 \mu \mathrm{m}$ which is the thickness of the sacrificial layer if the moving fingers are not sagging or curving due to residual stress. If the moving structures are sagging, the gap reduces to the depth of the dimples which is $0.75 \mu \mathrm{m}$ [65].

When a DC voltage $(\mathrm{V} \neq 0)$ is applied across the fixed unaligned fingers and fixed aligned fingers (including both fixed and moving fingers), a net repulsive electrostatic force is exerted on the moving aligned fingers. Figure 3-2 shows that the moving fingers of the actuator are raised to height, $H$, when a voltage is applied to the unaligned fixed fingers. The cross-section view of the actuator and the actuation mechanism for the repulsive force is shown in Figure 3-3. The 2D representation of the repulsive electrostatic forces is shown in Figure 3-4 using field lines.

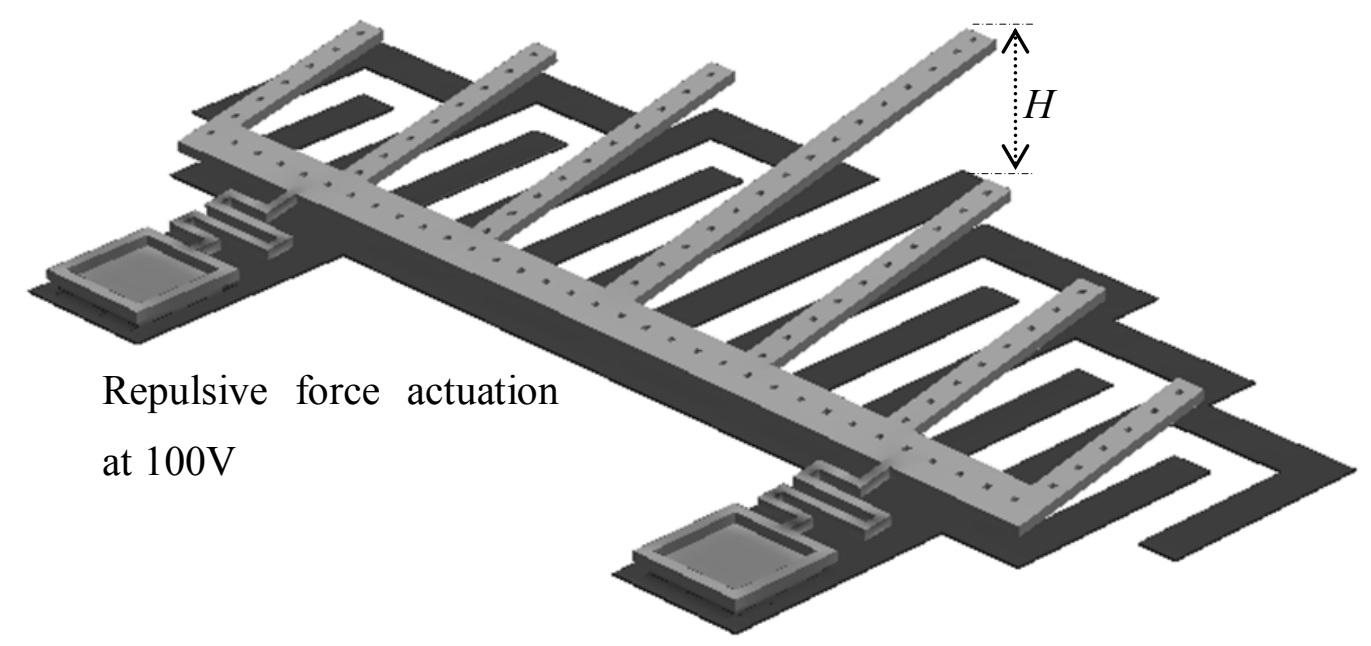

Figure 3-2 Illustrates electrostatic repulsive force actuator with $V \neq 0[63]$

\begin{tabular}{|c|c|c|c|c|}
\hline & GND & & GND & \\
\hline BNWWN: & & KWWN: & & NWWNW \\
\hline $\mathrm{V}$ & GND & $\mathrm{V}$ & GND & $\mathrm{V}$ \\
\hline $\mathrm{V}=$ voltage & $\mathrm{GND}=$ & & & \\
\hline
\end{tabular}

Figure 3-3 Actuation mechanism for repulsive electrostatic force [63] 


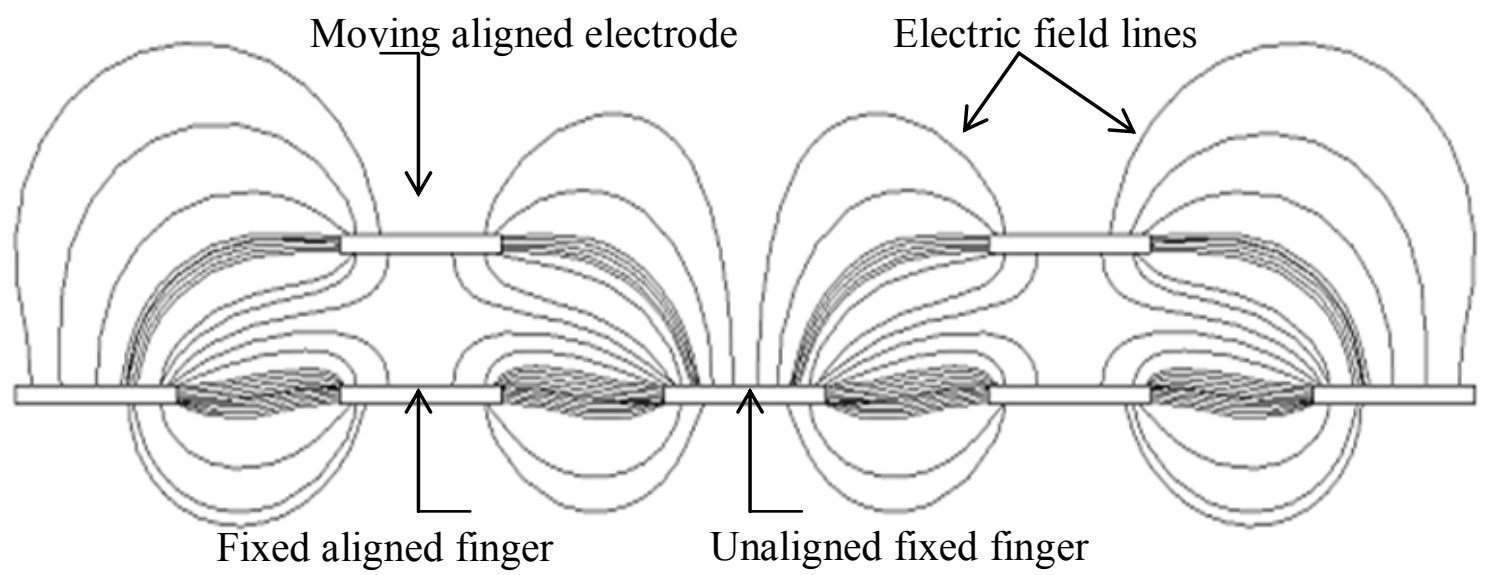

Figure 3-4 2D representation of repulsive electrostatic fields [68]

\subsubsection{Attraction Electrostatic Force Actuation}

The design can also generate attractive force if a voltage is applied to the unaligned fixed fingers and the fixed aligned fingers are assigned with floating potential. The actuation mechanism in this case is shown in Figure 3-5. Figure 3-6 shows the pattern of the electrostatic attraction force.

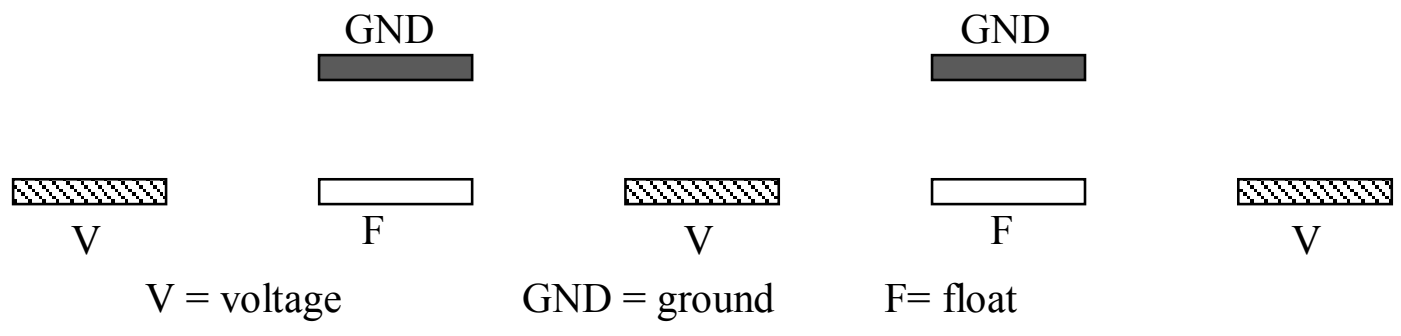

Figure 3-5 Actuation mechanism for attraction electrostatic force [68]

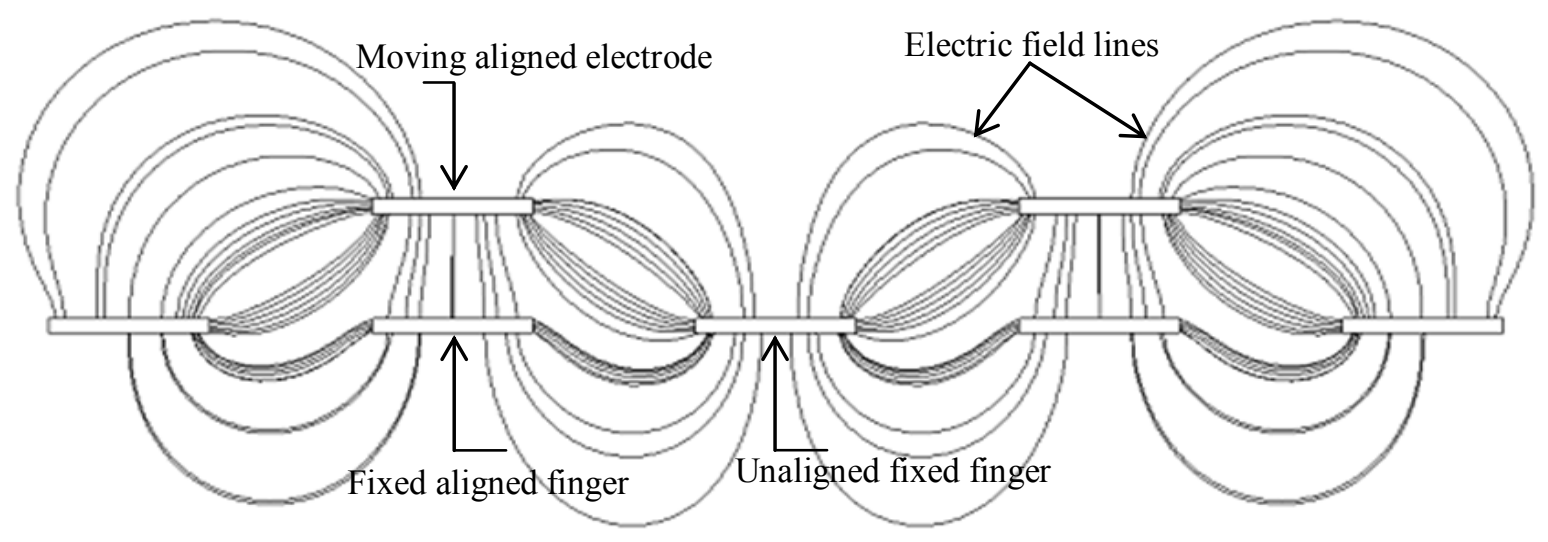

Figure 3-6 2D representation of attractive electrostatic fields [68] 


\subsubsection{Repulsive electrostatic force from capacitance}

The repulsive electrostatic actuator was previously developed by the authors of [62-64]. The equations of electrostatic repulsive force has been derived based on the capacitance between the moving fingers and the fixed unaligned fingers and fixed aligned fingers (Figure 3-7). Conformal mapping techniques were used to determine the capacitance under Drichilet and Neumann boundary conditions [62-64].

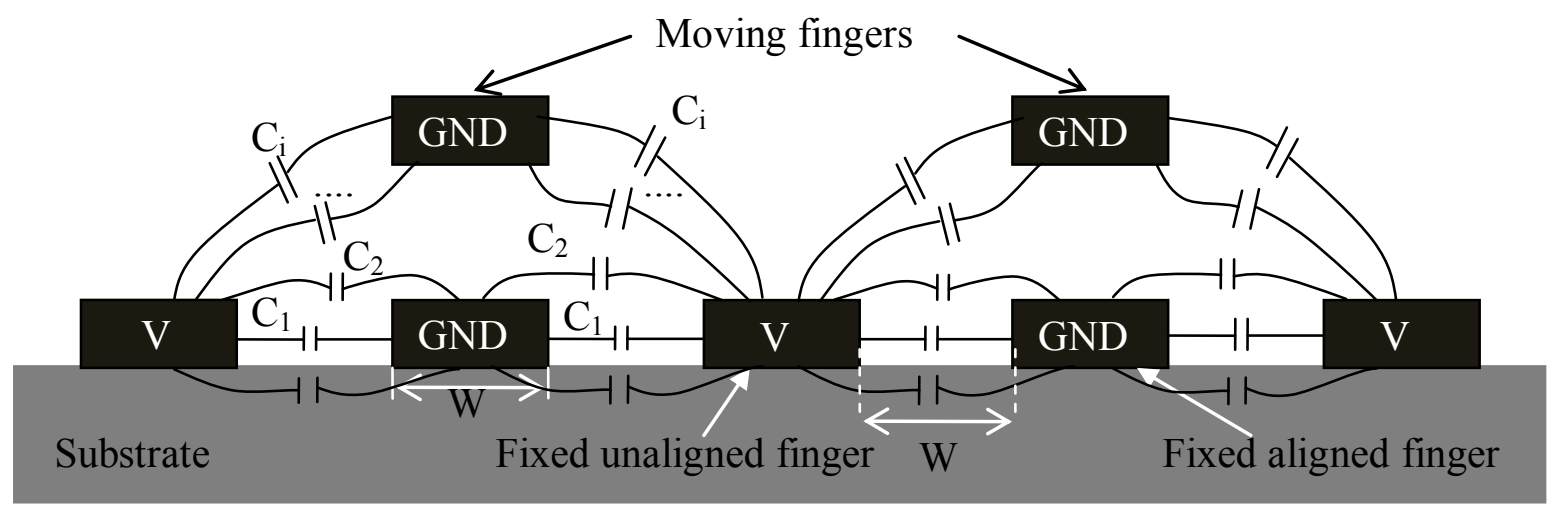

Figure 3-7 Illustration of the capacitance between the electrodes of repulsive actuator

When a voltage is applied across the fixed unaligned fingers, fringe capacitance linkup occurs between these fixed fingers and the moving fingers. The variation in the capacitance generates a repulsive electrostatic force on the moving fingers. According to potential theory [66], the differential energy stored is given by;

$$
d W=Q d V=C V d V
$$

where $W$ is the total energy in Joules, $C$ is the capacitance in Farads and $V$ is the applied actuation voltage in volts. The total capacitance per unit length between the fingers is the sum of the stray capacitances that couple the moving fingers and the fixed unaligned fingers. The repulsive electrostatic force and the torques on the fingers is given by [63]

$$
\begin{aligned}
& F_{\text {electrostaic }}=n V^{2} L_{f} \frac{d C}{d x}=\gamma \frac{d C}{d x} \\
& T=\int F_{\text {electrostaic }} l \cos \theta d l
\end{aligned}
$$


where $\gamma=n L_{f} V^{2}$ is constant for a given actuation voltage, the number of fingers and the length of moving fingers. The electrostatic force is proportional to the width of the moving fingers that generate maximum torque and the length of the moving fingers as well as the total number of the fixed unaligned fingers around the capacitor. The torque can be numerically obtained using the technique discussed in [64]. In order to obtain the maximum torque, the first derivative of the torque equation with respect to the displacement $x$ is set to zero. The resulting values of $x$ lead to the maximum torque generated [65].

Numerical and analytical results presented in [65] indicate that the finger length, rotation angle, finger width and the distance between the fingers all determine the maximum torque. In general, a maximum repulsive electrostatic force is achieved when the width of the fixed aligned fingers and the width of the unaligned fingers are approximately equal [63-65].

\subsubsection{PolyMUMPs process}

The PolyMUMPs [62] process is an eight-mask and seven physical layers micro-fabrication process of MEMSCAP (Figure 3-8). The process starts with a $675 \mu \mathrm{m}$ thick n-type 100 silicon substrate as the base material. Table 3-1 summarizes the thickness of the layers and the lithography levels.

Table 3-1 Layer name and lithography levels of PolyMUMPs process [62]

\begin{tabular}{lcccccccc}
\hline Layer name & Substrate & Nitride & Poly 0 & Oxide 1 & Poly 1 & Oxide2 & Poly 2 & Gold \\
\hline Thickness $(\mu \mathrm{m})$ & 675 & 0.6 & 0.5 & 2 & 2 & 0.75 & 1.5 & 0.5 \\
Lithography & --- & --- & HOLE0 & ANCHOR1, & HOLE1 & ANCHOR2, POLY & HOLE2 & HOLEM \\
level & & & & DIMPLE & & 1-POLY 2-VIA & & \\
\hline
\end{tabular}

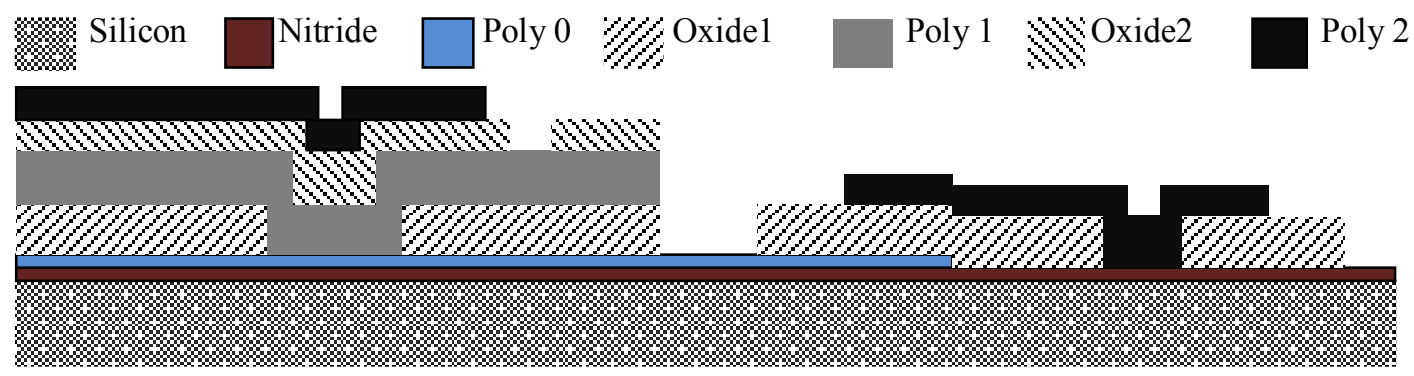

Figure 3-8 A schematic of the layers in PolyMUMPs process [62] 


\subsection{Tunable Capacitor Design}

The designs of two repulsive electrostatic force actuated tunable capacitors are presented in this section. The designs are shown in Figure 3-9 and 3-10 as Design 1 and Design 2 respectively. The dimensions of the designs are listed in Table 3-2 to 3.6.

Table 3-2 Actuator dimensions- Design 1

\begin{tabular}{llll}
\hline Parameter & Moving fingers & $\begin{array}{l}\text { Aligned fixed } \\
\text { fingers }\end{array}$ & $\begin{array}{l}\text { Unaligned fixed } \\
\text { fingers }\end{array}$ \\
\hline Finger length & $200,250,350,443$ & $200,250,350,443$ & $205,255,350,437$ \\
Finger width & 30 & 40 & 40 \\
\hline
\end{tabular}

Table 3-3 Actuator dimensions - Design 2

\begin{tabular}{llll}
\hline Parameter & Moving fingers & $\begin{array}{l}\text { Aligned fixed } \\
\text { fingers }\end{array}$ & $\begin{array}{l}\text { Unaligned fixed } \\
\text { fingers }\end{array}$ \\
\hline Finger length & $150,200,250,382$ & $150,200,250,382$ & $205,255,350,437$ \\
Finger width & 25 & 35 & 35 \\
\hline
\end{tabular}

Table 3-4 Capacitor plate area

\begin{tabular}{ll}
\hline Design 1 & $220 \mu \mathrm{m} \times 160 \mu \mathrm{m}$ \\
Design 2 & $300 \mu \mathrm{m} \times 302 \mu \mathrm{m}$ \\
\hline
\end{tabular}

Table 3-5 Anchoring (serpentine) springs

\begin{tabular}{lll}
\hline & Design-1 & Design-2 \\
\hline Width & 8 & 8 \\
Length & 88 & 88 \\
Turns & 2 & 2 \\
\hline
\end{tabular}

Table 3-6 Plate supporting springs

\begin{tabular}{lll}
\hline & Design-1 & Design-2 \\
\hline Width & 10 & 8 \\
Length & 92 & 92 \\
Turns & 2 & 2 \\
\hline
\end{tabular}

All dimensions are in micrometers. 


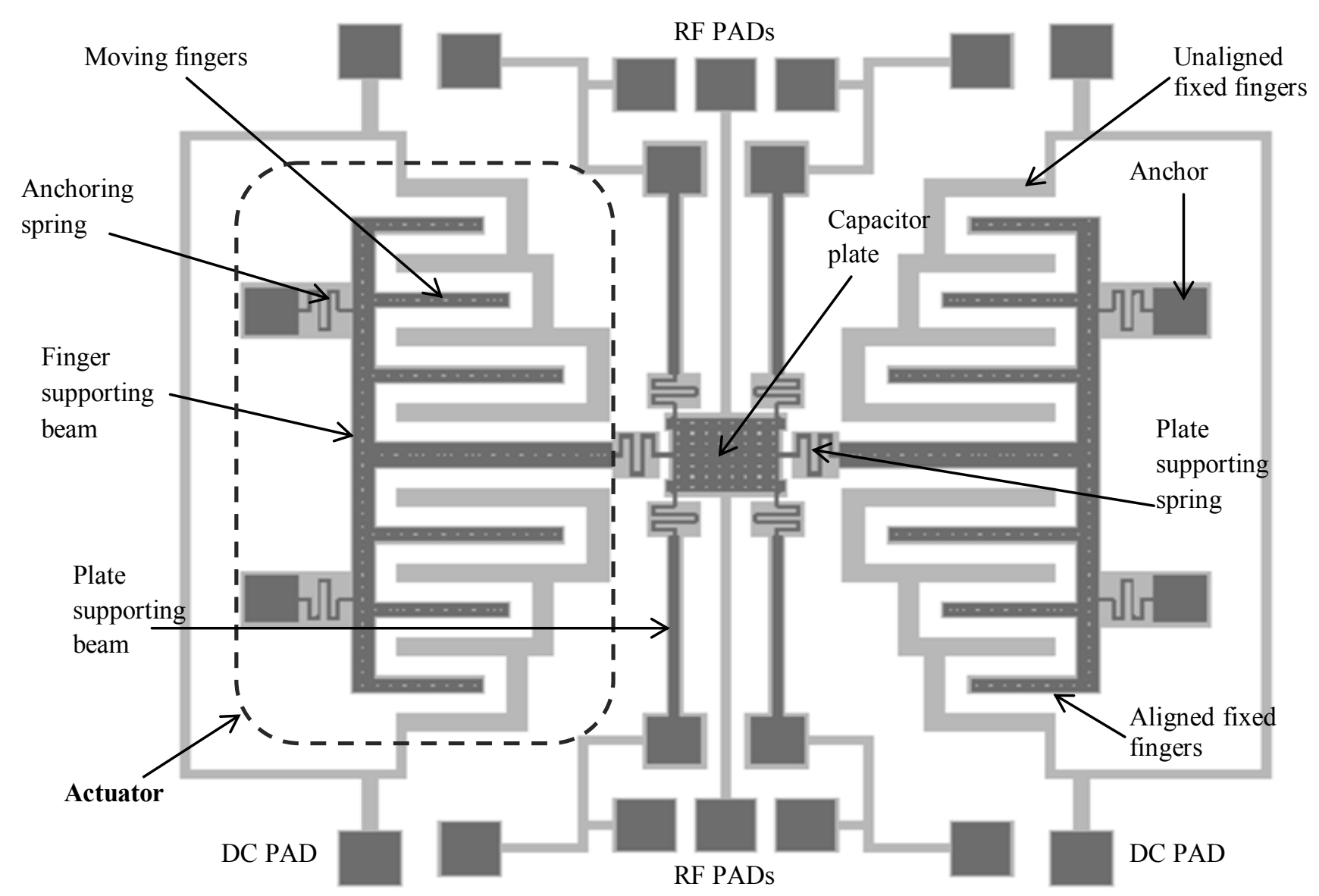

Figure 3-9 Translation type tunable capacitor (Design-1) 


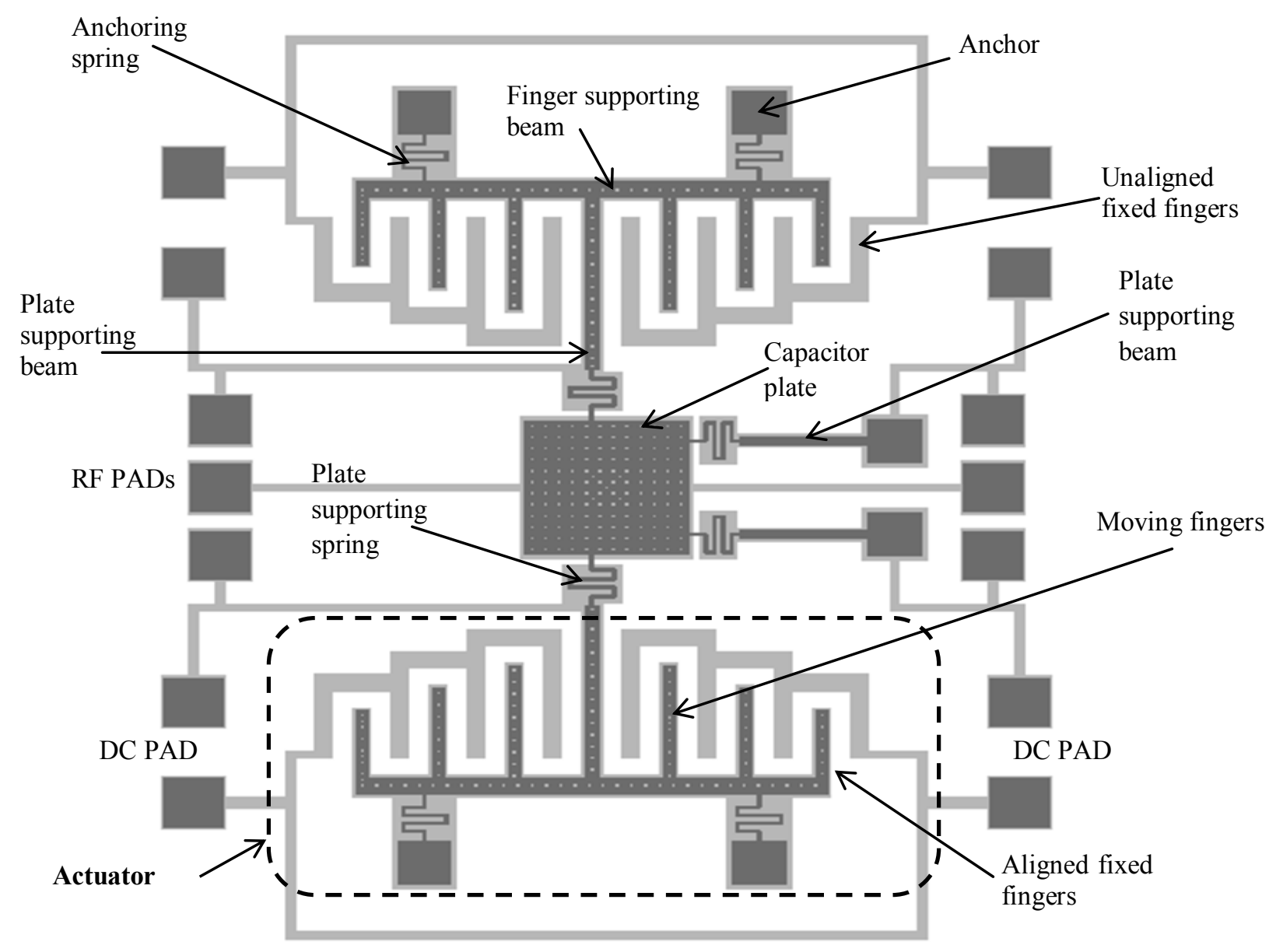

Figure 3-10 Rotation type tunable capacitor (Design-2) 
The repulsive electrostatic actuator consists of serpentine spring for anchoring, six fixed and six suspended finger electrodes that originate from the supporting beam. The Poly 0 layer is used for the fixed aligned and unaligned fingers of the actuator and the RF electrodes. The Poly 1 layer is used for the moving aligned fingers and the moving plate of the capacitor. The moving fingers of the actuator originate from a long beam of dimension $40 \mu \mathrm{m} \times 870 \mu \mathrm{m}$ that is anchored using serpentine anchoring springs.

The suspended/moving fingers are connected to the fixed aligned fingers through anchors for electromechanical interconnection as shown in Figure 3-9 and 3-10. The distance between the fixed aligned and the fixed unaligned fingers are numerically determined in such a way as to maximize the torque on the moving fingers. Maximum repulsive electrostatic force or torque is achieved when the width of the fixed aligned fingers and the width of the unaligned fingers are approximately equal [64]. The fixed unaligned fingers and the aligned fixed fingers are connected to pads for DC biasing and grounding, respectively.

\subsubsection{Principle of operation}

The principles of operation of the tunable capacitors entirely rely on the repulsive electrostatic actuator working principle. Referring to Figure 3-9 and Figure 3-10, when a DC voltage is applied across the actuator the moving fingers are pushed upward and out of plane. The moving plates of the capacitors are connected to the actuator with plate supporting springs so that the

plates move upward. As the voltage across the actuators increases the gap between the capacitor plates increases to tune the capacitance. The motion of the moving plates could be translation or rotation depending on the number and the position of the plate supporting beams. Capacitor Design 1 is translation type in which the capacitor plate moves vertically upward and parallel to the substrate or RF electrode. Capacitor Design 2 follows rotational motion i.e. the moving plate of the capacitor to the fixed electrode. The moving plate of Design 2 is initially parallel to the fixed electrode with a $2 \mu \mathrm{m}$ gap. However, during actuation it starts to rotate with an angle $\theta>0$.

\subsubsection{Spring design for the tunable capacitor}

Two serpentine springs are used in the designs: the anchoring spring and plate supporting spring. The dimensions of the serpentine spring (Figure 3-11) are selected based on sufficient stiffness and displacement using Coventor simulations. 


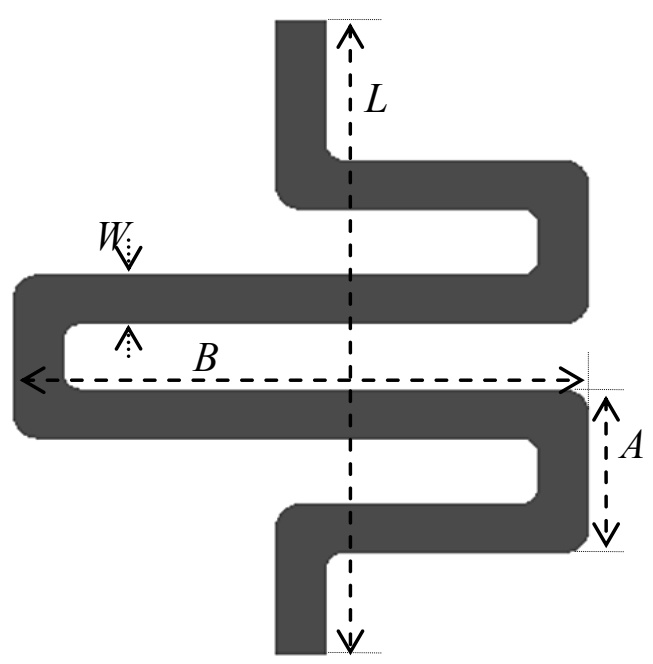

Figure 3-11 Serpentine spring

The stiffness of the anchoring spring determines the vertical displacement (range of motion) of the moving fingers/moving plate of the repulsive actuator. Therefore, the first step is to design the suitable actuator for sufficient displacement. The displacement of the moving plate increases with the actuator displacement. Micro-Newton forces are applied perpendicular to the tip of the spring to determine the stiffness of the serpentine spring. The total stiffness of the anchoring springs is four times the stiffness of the individual spring.

Table 3-7 Dimensions of anchoring serpentine spring

\begin{tabular}{l|l}
\hline Vertical length (A) & $26 \mu \mathrm{m}$ \\
Horizontal length (B) & $88 \mu \mathrm{m}$ \\
Thickness (t) & $2 \mu \mathrm{m}$ \\
Width (W) & $8 \mu \mathrm{m}$ \\
\hline
\end{tabular}

The displacement of the tip of the spring is obtained using simulation by applying a concentrated force at the tip of the spring and is shown in Table 3-8. In such case the spring constant can be calculated using the formula;

$$
F_{z i}=K_{z i} Z_{i}
$$


where $F_{z i}$ is the magnitude of the $i^{t h}$ concentrated force applied in the Z-direction, $K_{z i}$ is the spring constant corresponding to the applied force and at displacement of $Z_{i}$. The tip displacements $Z_{i}$, shown in Figure 3-12, are obtained using the CoventorWare [68] software.

Table 3-8 Simulated spring constant of the serpentine spring

\begin{tabular}{ccc}
\hline Force $\left(F_{z i}\right)(\mu \mathrm{N})$ & Tip displacement $\left(Z_{i}\right)(\mu \mathrm{m})$ & Spring constant $\left(K_{z i}\right)(\mathrm{N} / \mathrm{m})$ \\
\hline 1 & 1.1 & 0.909 \\
2 & 2.2 & 0.909 \\
3 & 3.3 & 0.909 \\
4 & 4.4 & 0.909 \\
\hline
\end{tabular}
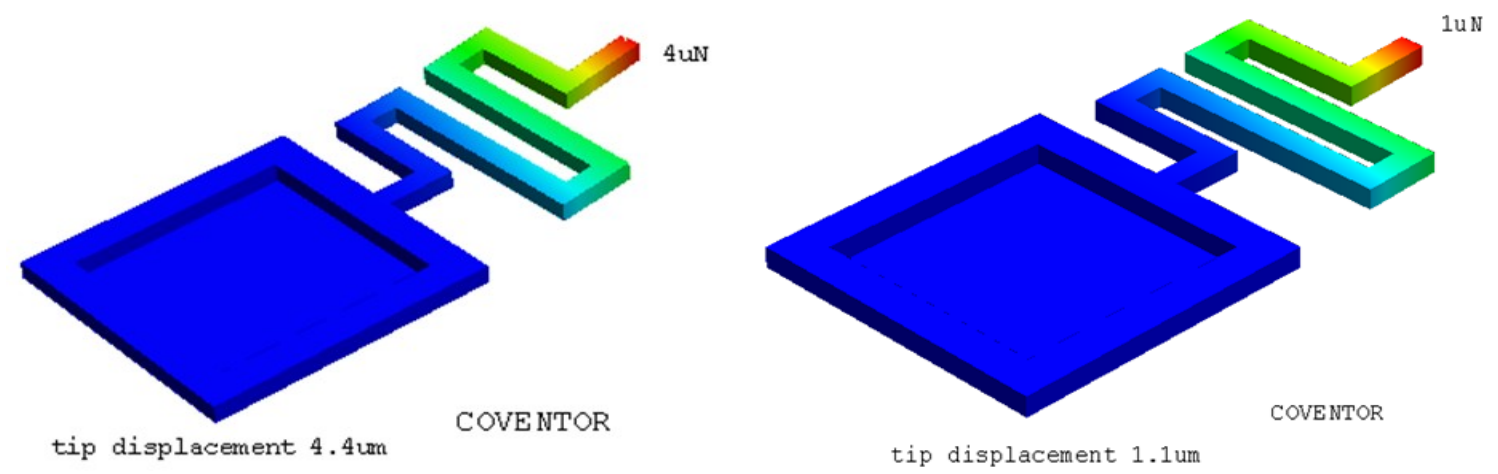

Figure 3-12 Simulated displacement of the tip of the anchoring springs at $1 \mu \mathrm{N}$ and $4 \mu \mathrm{N}$ forces

Spring constants of the moving fingers of the actuators are calculated assuming the fingers are free end cantilever beams. Table 3-9 shows the dimensions and stiffness of the moving fingers. The fingers are numbered from shortest finger to longest beam. The longest beam that supports the moving plate of the capacitor is called the longer beam. The longer beam has the lowest stiffness. Under the repulsive electrostatic actuation, fingers at the ends of the supporting beam deflect more than fingers around the middle of the beam. The fingers which are far away from the longer beam have less influence on the displacement of the moving plate. 
Table 3-9 Dimensions of the moving fingers and the corresponding stiffness

\begin{tabular}{cccc}
\hline Beams & Length & Width & Stiffness \\
\hline B1 & $150 \mu \mathrm{m}$ & $25 \mu \mathrm{m}$ & $9.48 \mathrm{~N} / \mathrm{m}$ \\
B2 & $200 \mu \mathrm{m}$ & $25 \mu \mathrm{m}$ & $4.0 \mathrm{~N} / \mathrm{m}$ \\
B3 & $250 \mu \mathrm{m}$ & $25 \mu \mathrm{m}$ & $2.048 \mathrm{~N} / \mathrm{m}$ \\
Longer beam & $382 \mu \mathrm{m}$ & $30 \mu \mathrm{m}$ & $0.689 \mathrm{~N} / \mathrm{m}$ \\
\hline
\end{tabular}
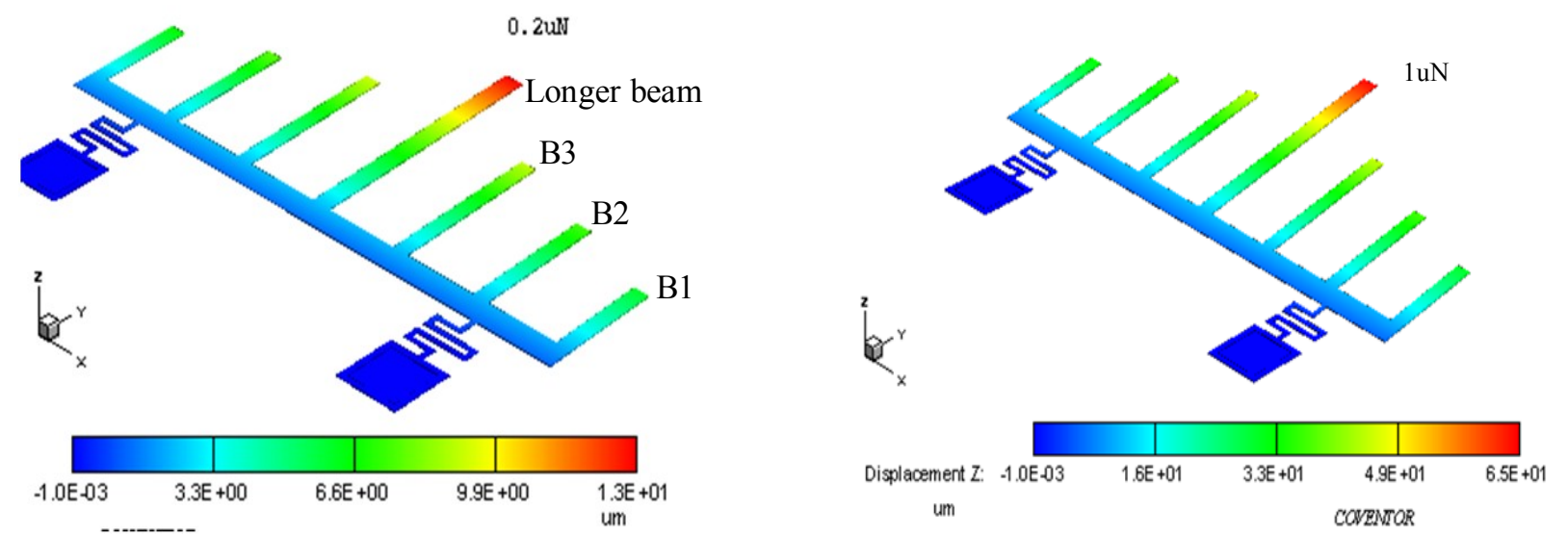

Figure 3-13 Deformation of repulsive actuator

\subsection{Capacitance equation}

In this section, the capacitance expression between the parallel plates of the capacitors is derived. The rotation of the actuators beam causes the moving plate to move upward in translation or rotation. The capacitance between the plates changes with the displacement of the actuators and the moving plate. The gap between the plates of the capacitor is proportional to the actuator displacement. The equations derived here do not account for fringing capacitances and thus these equations are not expected to be very accurate estimates of the capacitance.

\subsubsection{Translation type capacitor-Design 1}

In Design 1, Figure 3-14, the moving plate of the capacitor moves in linear manner. At any applied voltage the free body diagram including the middle longer beam, the plate supporting spring and the moving plate can be sketched as shown in Figure 3-14. Even though the actuator is rotating, the four plate-supporting beams allow the moving plate to follow a translational 
motion. Knowing the angle of rotation of the actuators and the total length including the length of the spring between the moving plate and the middle long beams, the displacement of the plate can be obtained. The total length from the base of the long beam to the edge of the moving plate of the capacitor is $L^{\prime}$.

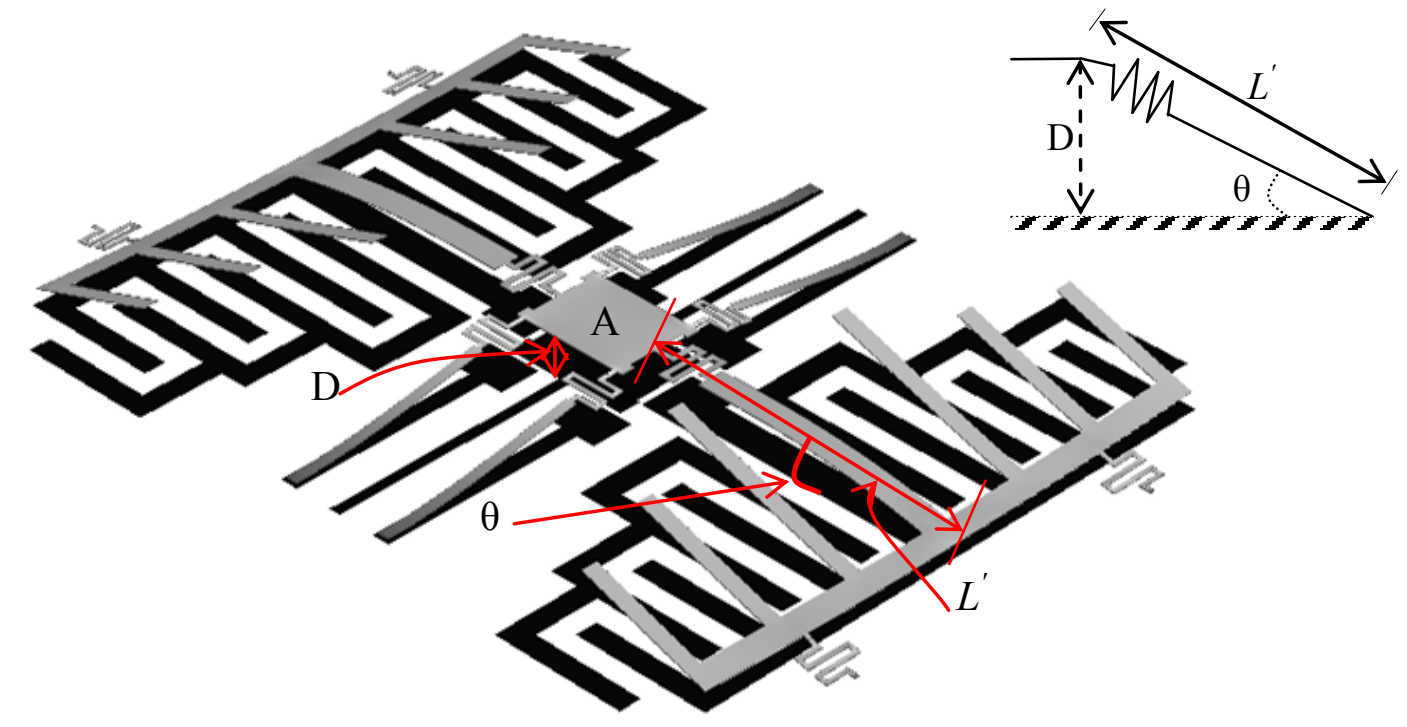

Figure 3-14 Translation type capacitor-Design 1

When a voltage $\mathrm{V}$ is applied across the actuators, the plate reaches a displacement of $D$ with actuator's rotation angle $\theta$. $D$ is expressed in terms of the angle $\theta$ and the length $L^{\prime}$ as;

$$
D=L^{\prime} \sin \theta
$$

The parallel plate capacitance is given by:

$$
C=\frac{\varepsilon A}{D} .
$$

where $C$ is the capacitance, $A$ the parallel plate area shown in Figure 3-14, and $\varepsilon$, the permittivity of the dielectric medium (in $F / m$ ).

The moving fingers of the actuators experience a rotation that is proportional to the angle of rotation and the rotational stiffness. The rotational torque is equal to the torque generated from 
the electrostatic force. The rotation angle of the actuator $\theta$ is determined using eqn. (3.7). The equation for the rotational torque is given by [64]:

$$
T_{\text {rotation }}=K_{\text {rotation }} \theta
$$

where $K_{\text {rotation }}$ is the rotational stiffness with units of $\mathrm{Nm} / \mathrm{rad}$ and $\theta$ the rotation angle in radians. For equilibrium to occur, the equations of the torque (3.3) and (3.7) must be equal any actuation voltage.

$$
\begin{gathered}
T=T_{\text {resistive }} \\
K_{\text {rotation }} \theta=\int_{0}^{L_{f}} F_{\text {electrostatic }} l \cos \theta d l
\end{gathered}
$$

The capacitance between the parallel plate electrodes is given by:

$$
C=\varepsilon A\left[L^{\prime} \sin \left(\frac{\int_{0}^{L_{f}} F_{\text {electrostatic }} l \cos \theta d l}{K_{\text {rotation }}}\right)\right]^{-1}
$$

\subsubsection{Rotation type capacitor-Design 2}

When the capacitor of Design 2, Figure 3-15 is actuated, the plate rotates with angle $\alpha$. The height of any point on the moving plate from the fixed plate will not remain constant and the plate becomes inclined with an angle $\alpha$. The free edge of the moving plate moves higher displacement compared with the anchored edge. As a result, the capacitance becomes the sum of the differential capacitance over the capacitor length $L$ as shown in Figure 3-15. In Figure 3-16 (a) and (b), the states of the moving plate are shown at rest and when actuated, respectively. 


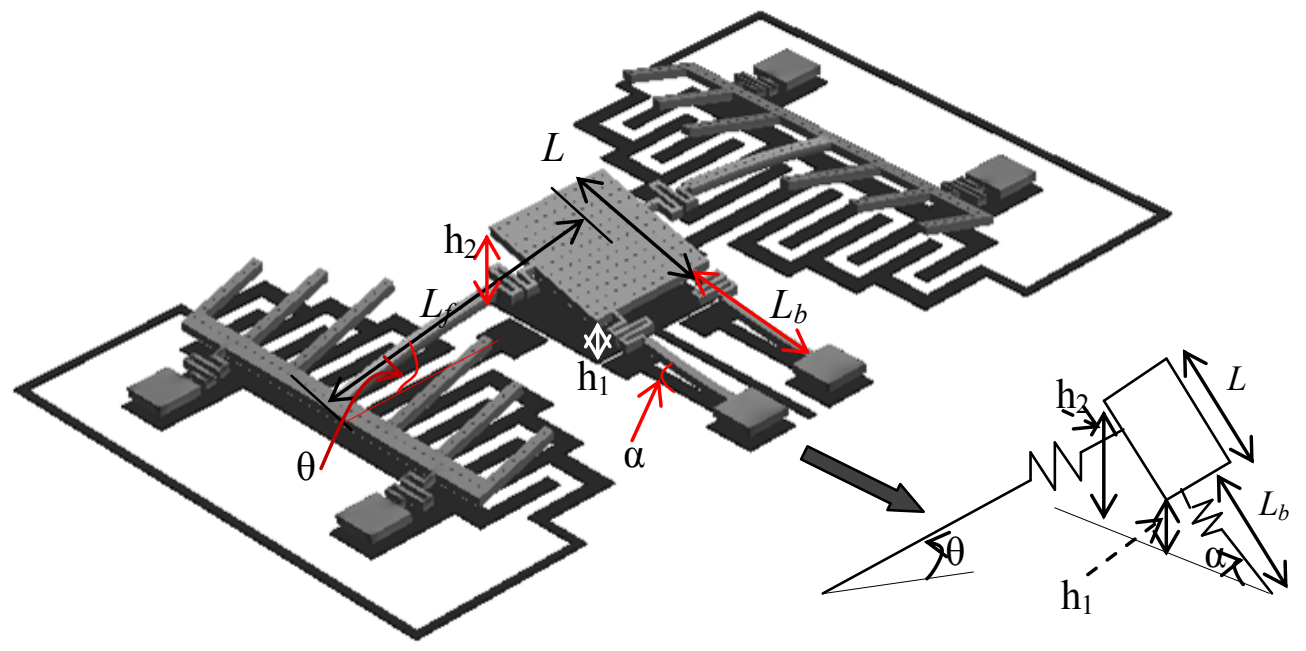

Figure 3-15 Rotation type capacitor-Design 2

Suppose that $L$ is the length of the moving plate, then $L+2 \Delta L$ is the length of the fixed plate, where $\Delta L$ is the enclosing length of the fixed plate (Poly 0) according to the PolyMUMPs fabrication process design rule. $L_{b}$ is the length of the beam supporting the plate from the anchor to the lower edge of the moving plate. $\alpha$ is the angle the plate supporting beams make with the horizontal when it starts rotating. $h_{1}$ and $h_{2}$ are the heights of the edges of the moving plate above the fixed plate during rotation. The values of $\mathrm{h}_{1}$ and $\mathrm{h}_{2}$ can be expressed in terms of $d$, the actuator displacement, and the rotation angle $\theta$.

The capacitance of such inclined plate may be found by transforming the z-plane to a t-plane (Figure 3-17) so that the ends of the ends of the plate are located at 1 and -1 respectively [69].

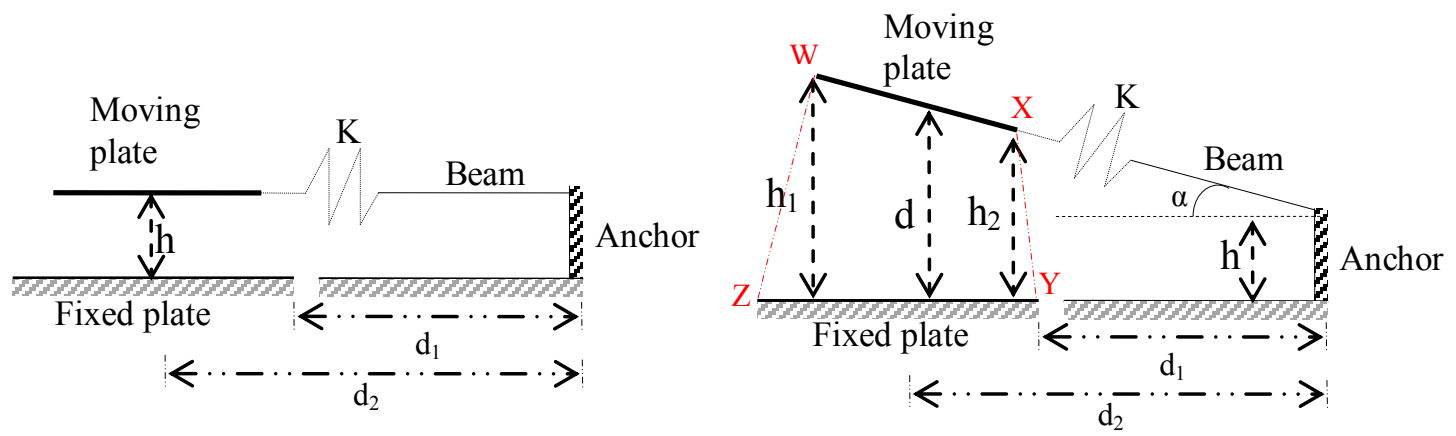

(a) State 1: $\alpha=0, \theta=0$ and $\mathrm{h}_{1}=\mathrm{h}_{2}=\mathrm{h}(\mathrm{V}=0)$

(b) State 2: $\alpha>0, \theta>0$ and $\mathrm{h}_{1}>\mathrm{d}>\mathrm{h}_{2}(\mathrm{~V} \neq 0)$

Figure 3-16 Cross section views of capacitor Design 2

The coordinate transformations of the plate are summarized in the table shown as: 
Table 3-10 Coordinate transformations

\begin{tabular}{|c|c|c|c|c|c|}
\hline \multirow{2}{*}{ Plane } & \multicolumn{4}{|c|}{ Coordinates } & \multirow{2}{*}{ Transformation } \\
\cline { 2 - 5 } & $\mathrm{W}$ & $\mathrm{X}$ & $\mathrm{Y}$ & $\mathrm{Z}$ & No \\
\hline$z$ & $\left(L_{b}+L\right) e^{j \alpha}$ & $L_{b} e^{j \alpha}$ & $d_{1}$ & $d_{1}+L+2 \Delta L$ & \multirow{2}{*}{$\left(\eta p_{1} z^{\frac{\pi}{\alpha}}+p_{2}, \alpha \rightarrow \pi\right.$} \\
\hline$t$ & $(-1,0)$ & $(\gamma, 0)$ & $(\eta, 0)$ & $(1,0)$ & $\xi=\frac{(1-\gamma)(1+t)}{2(t-\gamma)}$ \\
\hline$\xi$ & $(0,0)$ & $(-\infty, 0)$ & $\left(1 / k^{2}, 0\right)$ & $(1,0)$ & Schwarz-Christoffel \\
\hline$u$ & 0 & $i K^{\prime}$ & $K+i K^{\prime}$ & $K$ & \\
\hline
\end{tabular}

The cross section of the capacitors can be stretched to the t-plane as shown in Figure 3-17.

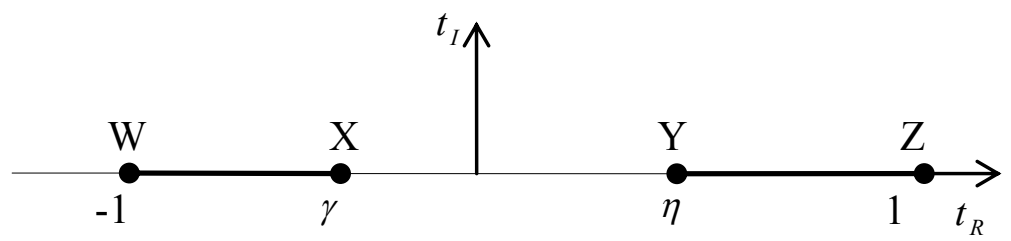

Figure 3-17 mapping of $z$-plane to $t$-plane

The coefficients $p_{1}$ and $p_{2}$ can be obtained by substituting for $z$ at $W$ and $Z$ and solving the resulting simultaneous equations:

$$
\left\{\begin{array}{c}
-1=-p_{1}\left(L_{b}+L\right)^{\frac{\pi}{\alpha}}+p_{2} \\
1=p_{1}\left(d_{1}+L+2 \Delta L\right)^{\frac{\pi}{\alpha}}+p_{2}
\end{array}\right.
$$

Solving eqn. (3.11) the values of the coefficients are:

$$
\begin{aligned}
& p_{1}=\frac{2}{\left(L_{b}+L\right)^{\frac{\pi}{\alpha}}+\left(d_{1}+L+2 \Delta L\right)^{\frac{\pi}{\alpha}}} \\
& p_{2}=\frac{\left(L_{b}+L\right)^{\frac{\pi}{\alpha}}-\left(d_{1}+L+2 \Delta L\right)^{\frac{\pi}{\alpha}}}{\left(L_{b}+L\right)^{\frac{\pi}{\alpha}}+\left(d_{1}+L+2 \Delta L\right)^{\frac{\pi}{\alpha}}}
\end{aligned}
$$




$$
t=\frac{2 z^{\frac{\pi}{\alpha}}+\left(L_{b}+L\right)^{\frac{\pi}{\alpha}}-\left(d_{1}+L+2 \Delta L\right)^{\frac{\pi}{\alpha}}}{\left(L_{b}+L\right)^{\frac{\pi}{\alpha}}+\left(d_{1}+L+2 \Delta L\right)^{\frac{\pi}{\alpha}}}
$$

The t-plane can be transformed to the $\xi$ plane for convenience. The $\xi$ plane has appropriate coordinates that are suitable for a Schwarz-Christoffel transformation [70, 71]. This transformation maps the interior of the plates to a rectangle using the equation:

$$
\begin{gathered}
\xi k^{2}-1=0 \ldots \ldots \\
k=\sqrt{\frac{2(\eta-\gamma)}{(1-\gamma)(1+\eta)}} .
\end{gathered}
$$

The parameters $\eta$ and $\gamma$ can be expressed in terms of the geometric parameters of the capacitor as:

$$
\begin{gathered}
\gamma=\frac{-2 L_{b}^{\frac{\pi}{\alpha}}+\left(L_{b}+L\right)^{\frac{\pi}{\alpha}}-\left(d_{1}+L+2 \Delta L\right)^{\frac{\pi}{\alpha}}}{\left(L_{b}+L\right)^{\frac{\pi}{\alpha}}+\left(d_{1}+L+2 \Delta L\right)^{\frac{\pi}{\alpha}}} \\
\eta=\frac{2 d_{1}^{\frac{\pi}{\alpha}}+\left(L_{b}+L\right)^{\frac{\pi}{\alpha}}-\left(d_{1}+L+2 \Delta L\right)^{\frac{\pi}{\alpha}}}{\left(L_{b}+L\right)^{\frac{\pi}{\alpha}}+\left(d_{1}+L+2 \Delta L\right)^{\frac{\pi}{\alpha}}} .
\end{gathered}
$$

The upper half of $\xi$ plane is transformed into the interior of a rectangle in the u-plane by the Schwarz-Christoffel transformations as:

$$
u=c k \int_{0}^{\xi} \frac{1}{\sqrt{\xi(1-\xi)\left(1-\xi k^{2}\right)}} d \xi+c_{0}
$$

$c$ and $c_{0}$ can be determined from the constraints at $\mathrm{W}$ and $\mathrm{Z}$ to yield $c_{0}=0$ and $c k=1$. If $\xi=\tau^{2}$, and substituting the conditions at $W$ and $Z$

$$
K(k)=\int_{0}^{1} \frac{1}{\sqrt{\left(1-\tau^{2}\right)\left(1-k^{2} \rho^{2}\right)}} d \rho
$$




$$
i K^{\prime}(k)=\int_{1}^{\frac{1}{k}} \frac{1}{\sqrt{\left(1-\tau^{2}\right)\left(1-k^{2} \rho^{2}\right)}} d \rho
$$

where $K(k)$ is the complete elliptic integral of the first kind with modulus $k \cdot K\left(k^{\prime}\right)=K^{\prime}(k)$ and

$$
\begin{gathered}
k^{\prime}=\sqrt{1-k^{2}} \\
k^{\prime}=\sqrt{\frac{(1+\gamma)(1-\eta)}{(1+\eta)(1-\gamma)}} .
\end{gathered}
$$

The total capacitance can be expressed as;

$$
C=C_{\text {inside }}+C_{\text {outside }}=\varepsilon_{r} \varepsilon_{0}\left(\frac{K^{\prime}\left(k_{\text {inside }}\right)}{K\left(k_{\text {inside }}\right)}+\frac{K^{\prime}\left(k_{\text {outside }}\right)}{K\left(k_{\text {outside }}\right)}\right)
$$

$k_{\text {inside }}$ and $k_{\text {outside }}$ represent the $k$ values inside the plates and outside the plates for the angles $\alpha$ and $2 \pi-\alpha$ respectively.

$$
\begin{aligned}
& k_{\text {inside }}=\sqrt{\frac{\left(d_{1}^{\frac{\pi}{\alpha}}+L_{b}^{\frac{\pi}{\alpha}}\right)\left(\left(L_{b}+L\right)^{\frac{\pi}{\alpha}}+\left(d_{1}+L+2 \Delta L\right)^{\frac{\pi}{\alpha}}\right)}{\left(d_{1}^{\frac{\pi}{\alpha}}+\left(L_{b}+L\right)^{\frac{\pi}{\alpha}}\right)\left(L_{b}^{\frac{\pi}{\alpha}}+\left(d_{1}+L+2 \Delta L\right)^{\frac{\pi}{\alpha}}\right)} .} \\
& k_{\text {inside }}^{\prime}=\sqrt{\frac{\left(\left(L_{b}+L\right)^{\frac{\pi}{\alpha}}-L_{b}^{\frac{\pi}{\alpha}}\right)\left(\left(d_{1}+L+2 \Delta L\right)^{\frac{\pi}{\alpha}}-d_{1}^{\frac{\pi}{\alpha}}\right)}{\left(\left(L_{b}+L\right)^{\frac{\pi}{\alpha}}+d_{1}^{\frac{\pi}{\alpha}}\right)\left(L_{b}^{\frac{\pi}{\alpha}}+\left(d_{1}+L+2 \Delta L\right)^{\frac{\pi}{\alpha}}\right)}} . \\
& k_{\text {outside }}=\sqrt{\frac{\left(d_{1}^{\frac{\pi}{2 \pi-\alpha}}+L_{b}^{\frac{\pi}{2 \pi-\alpha}}\right)\left(\left(L_{b}+L\right)^{\frac{\pi}{2 \pi-\alpha}}+\left(d_{1}+L+2 \Delta L\right)^{\frac{\pi}{2 \pi-\alpha}}\right)}{\left(d_{1}^{\frac{\pi}{2 \pi-\alpha}}+\left(L_{b}+L\right)^{\frac{\pi}{2 \pi-\alpha}}\right)\left(L_{b}^{\frac{\pi}{2 \pi-\alpha}}+\left(d_{1}+L+2 \Delta L\right)^{\frac{\pi}{2 \pi-\alpha}}\right)}} \\
& k_{\text {outside }}^{\prime}=\sqrt{\frac{\left(\left(L_{b}+L\right)^{\frac{\pi}{2 \pi-\alpha}}-L_{b}^{\frac{\pi}{2 \pi-\alpha}}\right)\left(\left(d_{1}+L+2 \Delta L\right)^{\frac{\pi}{2 \pi-\alpha}}-d_{1}^{\frac{\pi}{2 \pi-\alpha}}\right)}{\left(\left(L_{b}+L\right)^{\frac{\pi}{2 \pi-\alpha}}+d_{1}^{\frac{\pi}{2 \pi-\alpha}}\right)\left(L_{b}^{\frac{\pi}{2 \pi-\alpha}}+\left(d_{1}+L+2 \Delta L\right)^{\frac{\pi}{2 \pi-\alpha}}\right)}}
\end{aligned}
$$

The angle $\alpha$ can be found from the dimensions shown in Figure 3-17 (b) using the equation: 


$$
\alpha=\tan ^{-1}\left(\frac{d-h}{\frac{L}{2}+\Delta L+d_{1}}\right)
$$

The distance $\mathrm{d}$ is expressed in a similar way as eqn. 3-10 and it is

$$
d=L_{f} \sin \theta
$$

$\theta$ is the rotation angle of the actuator at the actuation voltage. $L_{f}$ is the length of the plate supporting plate, the length of plate supporting spring and half length of the moving plate. When the angle $\alpha=0$, then eqn. (3.24) reduces to the conventional parallel plate capacitor equation.

\subsection{Simulations}

3D models of the translation and the rotation type tunable capacitors are generated using the PolyMUMPs process of CoventorWare [68]. In the coupled-electromechanical simulation of the designs, voltages were applied to find out the displacements of the moving plate of the capacitor. At no voltage the maximum gap between the moving plate and the fixed electrode is $2 \mu \mathrm{m}$ based on the PolyMUMPs fabrication process, assuming the moving plate and the moving fingers do not sag down. Otherwise, the gap will reduce to the depth of the dimples on the moving layer (Poly 1). For nonzero voltages, the repulsive electrostatic force generated acts on the moving fingers to lift the moving plate of the capacitor so that capacitance changes. The top surface of the fixed electrode on the substrate is taken as a reference plane to measure the displacements of the moving plate. The graph of Figure 3-18 shows the displacement of the moving plate vs. actuation voltages from $0 \mathrm{~V}$ to $200 \mathrm{~V}$ without RF DC equivalent voltage of the RF signal. At $160 \mathrm{~V}$, the displacement obtained is $6.9 \mu \mathrm{m}$. Figure 3-20 and Figure 3-21 illustrate the deformations of Design 1 and Design 2, respectively at 160V. The top surface of the fixed electrode is the reference to measure the displacement of the moving plate.

The simulated displacements and capacitances for Design1 and Design 2 are presented in Table 3-11 and Table 3-12, respectively. The values in the tables show the upward displacements of the moving plate from the top surface of the fixed electrode for $20 \mathrm{~V}$ to $200 \mathrm{~V}$. The simulated capacitances include the fringing capacitance between the fixed electrode and the nearby fingers of the actuators. In the capacitance simulation, the parasitic capacitance through the silicon- 
nitride and the silicon substrate are not included as these structures are hidden from the simulator to reduce the computational time.

The presence of holes affect the stiffness of the moving plates and the moving fingers of the actuators. The maximum displacement obtained for capacitor Design 2 without holes at $100 \mathrm{~V}$ is $5.08 \mu \mathrm{m}$ whereas the displacement with holes is increased to the $5.69 \mu \mathrm{m}$. The change in the displacement due to the presence of holes resulted in $0.61 \mu \mathrm{m}$ which resulted in a $10.72 \%$ error. The inclusion of the dimples causes non-uniform surface and an extended feature at the bottom surface of the moving fingers and the moving plate that causes convergence error in the simulation.

Figure 3-18 and 3-19 show the simulated capacitance and the displacements corresponding to the applied voltage, respectively. From the graphs it seen that capacitance decreases as the tuning gap increases because the moving plate moves up when the driving voltage is applied. The deformation of the capacitors Design 1 and 2 are shown in Figure 3-20 and 3-21.

Table 3-11 Simulation results--Design 1 without RF power

\begin{tabular}{lllllllllll}
\hline Voltage $(\mathrm{V})$ & 20 & 40 & 60 & 80 & 100 & 120 & 140 & 160 & 180 & 200 \\
Displacement $(\mu \mathrm{m})$ & 0.13 & 0.51 & 1.2 & 2.0 & 3.1 & 4.2 & 5.4 & 6.6 & 7.8 & 9.0 \\
Capacitance $(\mathrm{fF})$ & 146.25 & 124.11 & 97.35 & 77.88 & 61.08 & 50.24 & 42.09 & 36.22 & 31.78 & 28.32 \\
\hline
\end{tabular}

Table 3-12 Simulation results--Design 2-without RF power

\begin{tabular}{lllllllllll}
\hline Voltage $(\mathrm{V})$ & 20 & 40 & 60 & 80 & 100 & 120 & 140 & 160 & 180 & 200 \\
Displacement $(\mu \mathrm{m})$ & 0.19 & 0.8 & 1.8 & 3.3 & 4.9 & 6.6 & 8.3 & 9.9 & 11 & 13 \\
Capacitance (fF) & 366.2 & 286.43 & 211.05 & 151.32 & 116.23 & 93.26 & 77.86 & 67.39 & 61.69 & 53.47 \\
\hline
\end{tabular}




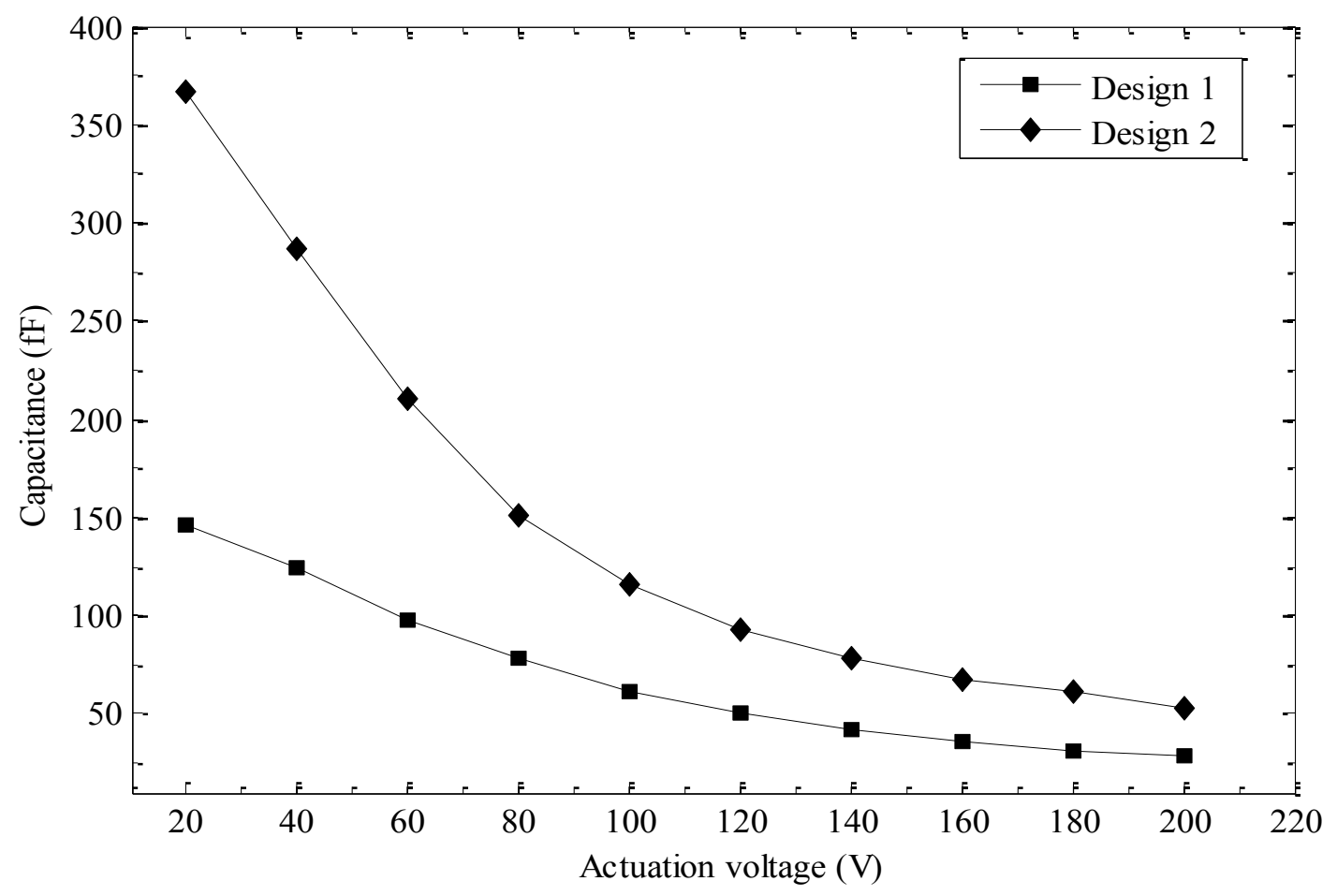

Figure 3-18 Simulated capacitance vs. voltage for Design 1and design 2

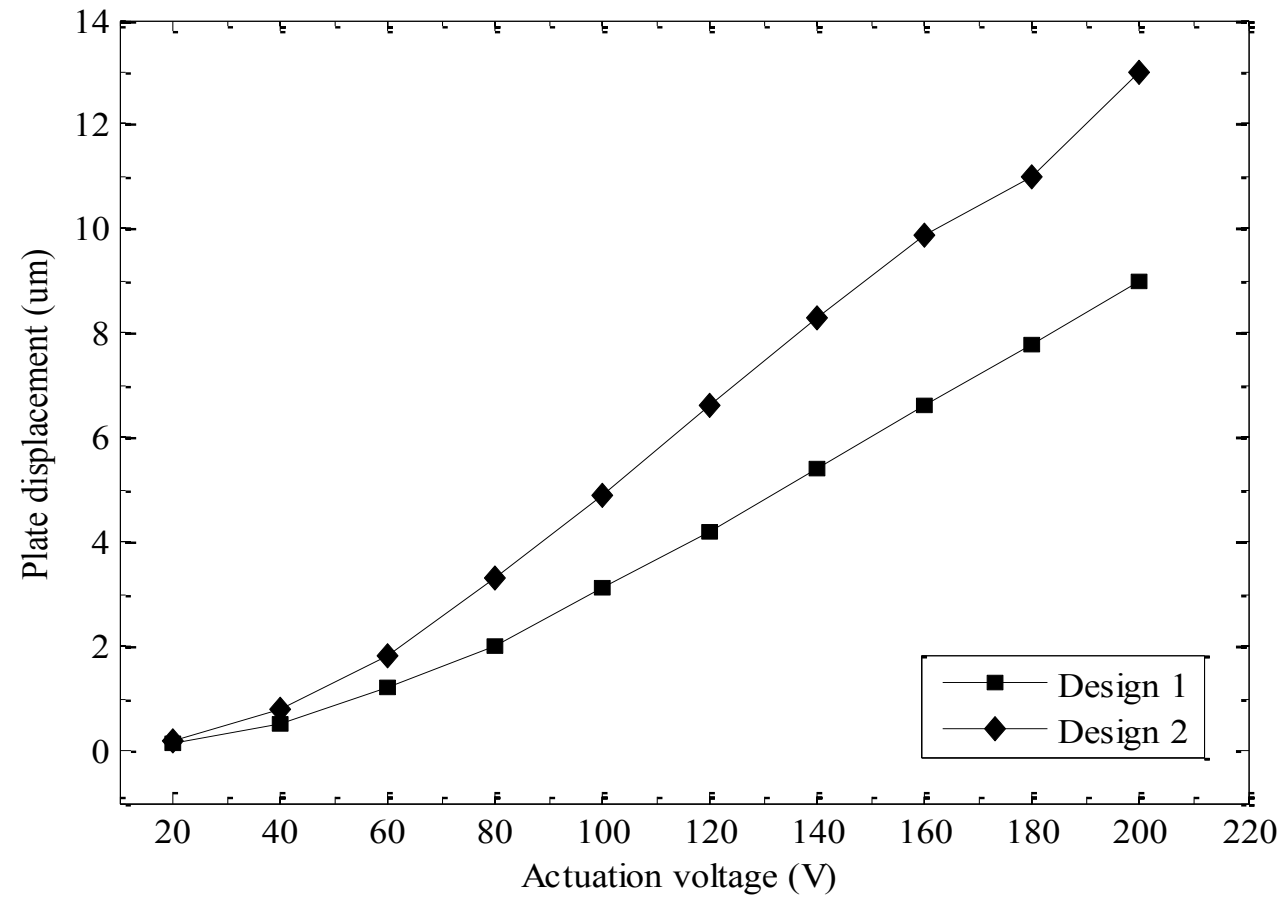

Figure 3-19 Displacement vs. voltage for Design 1and Design 2 


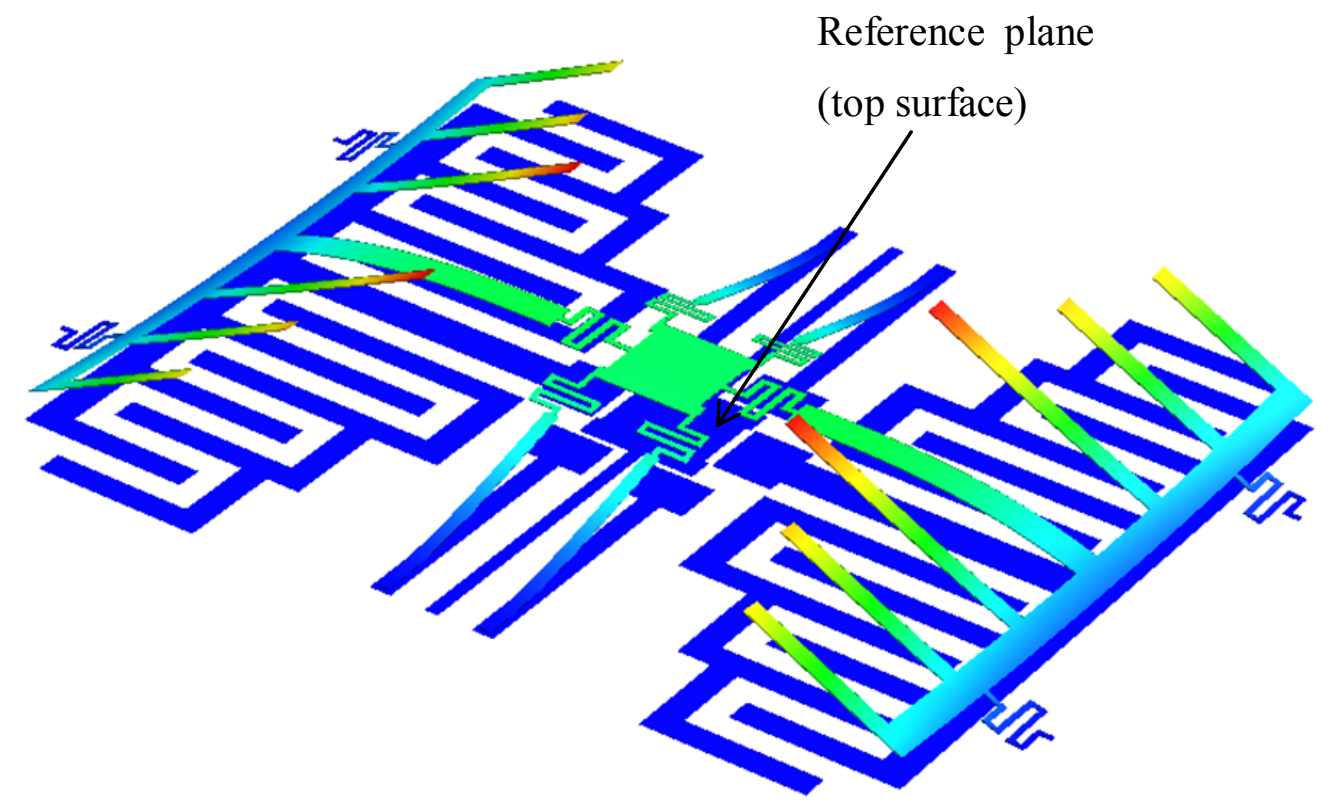

Figure 3-20 Tuning of capacitor-Design 1

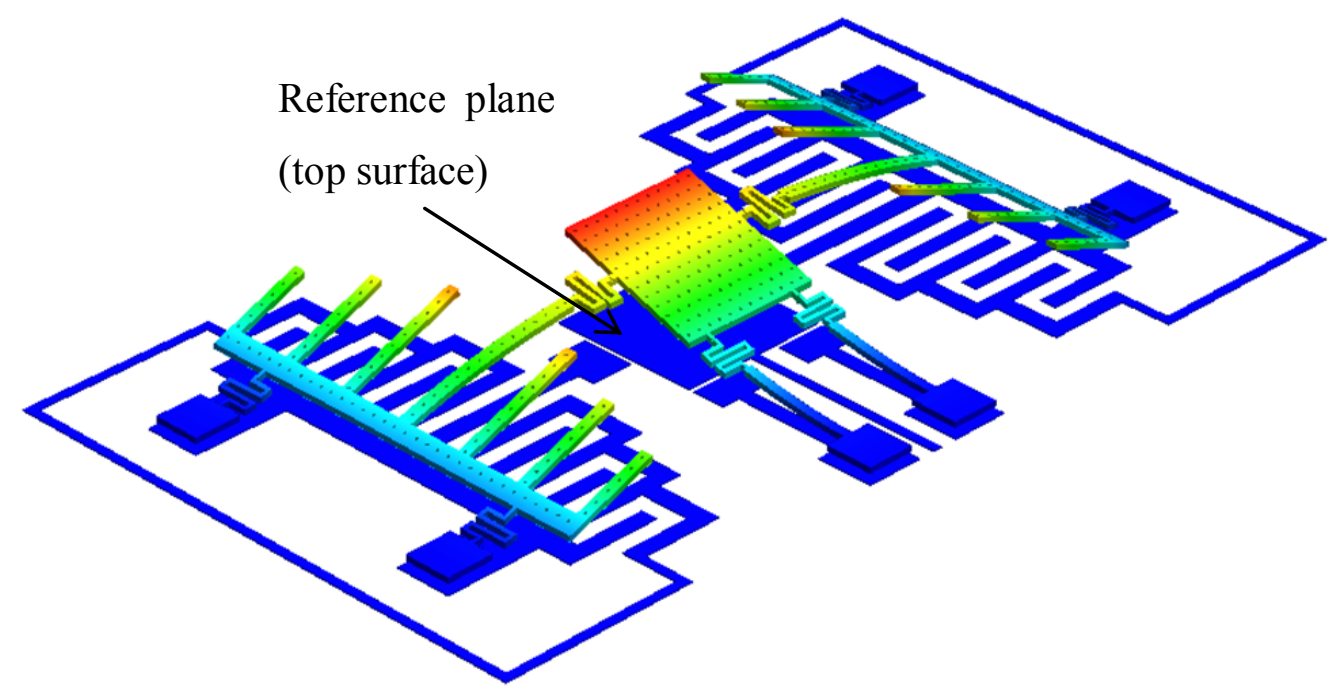

Figure 3-21 Tuning of capacitor-Design 2

The tunable capacitors performances are highly dependent on the repulsive actuator that drives the moving plate upward. The stiffness of the actuators i.e. the moving fingers are the most important parameters that control the displacement of the moving plate. Low stiffness results in high displacement of the moving plate. Long and narrow fingers give lower stiffness, thus lower 
stiffness of the entire capacitors are achievable. However, the repulsive electrostatic force is not strong enough balance the attraction force generated from the RF power. Lower stiffness of the fingers and the capacitor cause sagging effect on fabricated prototypes and thus the moving plate could mechanically contact the fixed plates.

Performance parameters, capacitance and quality factors are the parameters that determine the design of the tunable capacitors. Large parallel plate area provides high capacitance density. On the other hand, the quality factor will be reduced due the capacitive impedance. Small capacitance values produce high quality factors.

The resistance of the RF electrodes which is a function of geometry, affects the quality factors and power loss. Large electrodes and RF pad sizes lower the resistance loses.

\subsection{RF power and tuning ratio of repulsive capacitors}

In this section the effect of the RF signal on the tuning ratio of the capacitor is presented. The RF power induces its own DC voltage which pulls the moving plate of the tunable capacitors and brings down the maximum displacement that could have been achieved without RF power. The impact of the RF power on the tuning ratio is analyzed for both designs.

\subsubsection{Interaction of RF power and actuation voltage}

The instantaneous RF voltage is given by

$$
v_{R F}(t)=v_{\text {max }} \sin (\omega t)
$$

where $v(t)$ is the instantaneous RF voltage at any time $t, v_{\max }$ is the amplitude and $\omega$ is the radian frequency of the signal. The RF signal induces a root mean square (RMS) voltage on the RF electrode of the tunable capacitor.

$$
V_{R M S}=v_{\max } / \sqrt{2} .
$$

The RMS voltage of the RF signal pulls the moving plate of the capacitor down and tends to reduce the gap. Therefore, the RMS voltage of the RF signal, its induced attractive force across the plates of the capacitor, the actuation voltage and its generated repulsive force interact with each other to tune the capacitance. 
In order to estimate the minimum actuation voltage that prevents the moving plate of the capacitor from touching the fixed plate, the RMS voltage is fixed while changing the actuation voltage. Hence, the capacitances are obtained from the simulation for different actuation voltages with fixed RMS voltage of the RF signal. In the analysis, it was observed that lower RF voltage levels induce small changes on the gap of the plates of the capacitor. If the actuation voltages are not strong enough to keep the moving plate, large RF power could pull the moving plate further down towards the fixed electrode. Therefore, for high power RF signal, non-zero minimum actuation voltage is required to avoid contact between the plates.

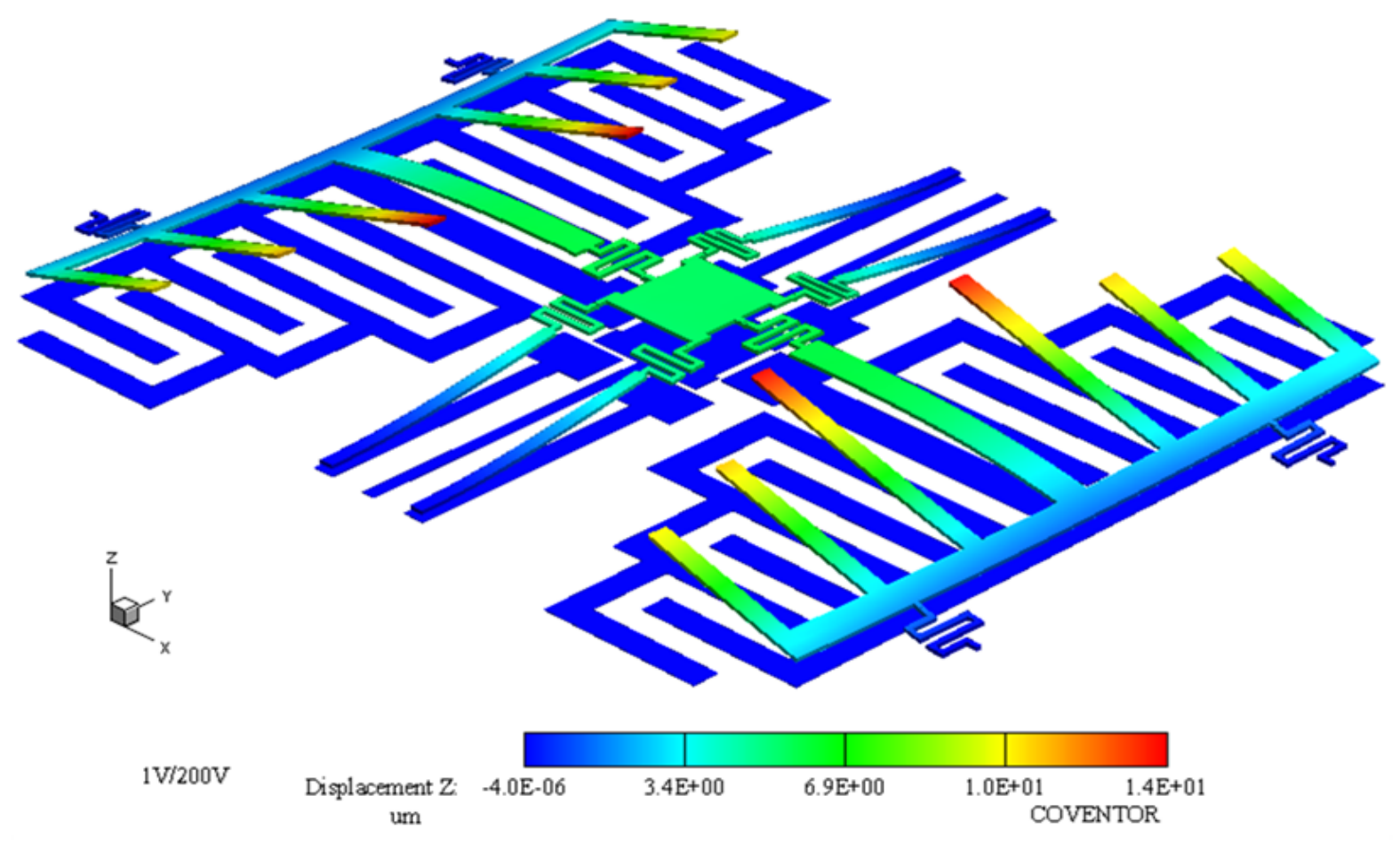

Figure 3-22 Simulated capacitor of Design 1at 1V DC RF equivalent voltage

Figure 3-22 shows the deformation of capacitor Design 1 at $1 \mathrm{~V}$ RMS RF equivalent and 200V actuation. Without the RF voltage the maximum displacement of the plate of the capacitor is $9 \mu \mathrm{m}$. When RF DC voltage equivalent of $1 \mathrm{~V}$ was applied to the RF electrode, the maximum displacement reduced to $5.58 \mu \mathrm{m}$. 


\subsubsection{Tuning ratio in the presence of RF power}

The RF power significantly influences the displacement of the moving plate and thus the tuning ratios of the capacitors. Tuning ratio of the capacitors is proportional to the displacements of the moving plate. RF equivalent voltages of $1 \mathrm{~V}, 2 \mathrm{~V}, 3 \mathrm{~V}, 4 \mathrm{~V}$ and $5 \mathrm{~V}$ are applied to the capacitors to analyze the tuning ratio. When the actuation voltage is $0 \mathrm{~V}$, the moving plate is free to move down due to the attractive force of the RF power. The gap between the plates of the capacitor will be below its nominal value $(\sim 2 \mu \mathrm{m})$. Consequently, the initial capacitance exceeds the capacitance value at the nominal gap. As the actuation voltage increases for a given RF equivalent voltage, the moving plate starts to move away from the fixed plate. The simulated capacitances are extracted to spread sheet and plotted in Matlab [72]. Figure 3-23 shows the simulated capacitance curves for RF DC equivalent voltage of $0 \mathrm{~V}, 1 \mathrm{~V}, 2 \mathrm{~V}, 3 \mathrm{~V}$ and $4 \mathrm{~V}$.

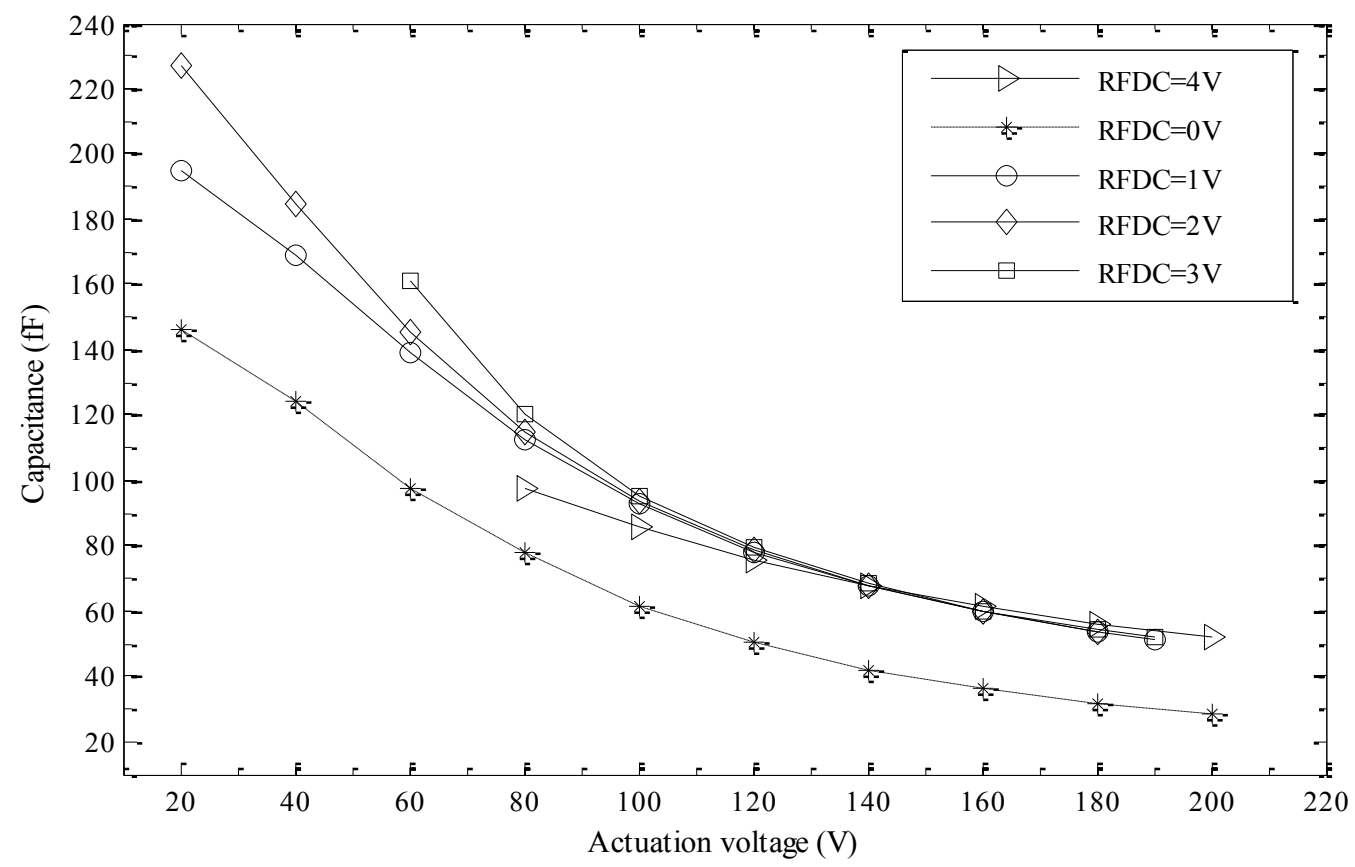

Figure 3-23 Simulated capacitance Vs actuation and RFDC equivalent voltages-Design 1

As the RF DC equivalent voltage increases with a $1 \mathrm{~V}$ step the initial capacitance is increases. This is because the RF DC equivalent voltage pulls down the moving plate further down and reduces the gap to the height of the dimples. Actuation voltages below $80 \mathrm{~V}$ are not strong enough to balance a $4 \mathrm{~V}$ RF signal. Until the actuation voltages exceed the $80 \mathrm{~V}$, the capacitance 
tuning ratio at $4 \mathrm{~V}$ RF signal is nearly unity which is non-tunable. At $0 \mathrm{~V}$ actuation and $4 \mathrm{~V}$ RF DC equivalent, the capacitance can be calculated using the parallel plate equation (3.6). The gap between the plates, in this case, is the height of the dimples. The calculated capacitance when the gap is $0.75 \mu \mathrm{m}$ is $415.4 \mathrm{fF}$. The minimum capacitance at $4 \mathrm{~V}$ RF DC from the graph of Figure 3-23 is $52 \mathrm{fF}$. From the above simulations tuning ratios for $1 \mathrm{~V}, 2 \mathrm{~V}, 3 \mathrm{~V}$ and $4 \mathrm{~V}$ RF DC are $358.2 \%$, $438.5 \%$ and $307.6 \%$ and $188.6 \%$, respectively.

Similarly, the tuning ratio of the incline plate capacitor (Design 2) is simulated for $2.5 \mathrm{~V}$ RF DC equivalent voltage. Since the plate has large area, pull-in occurs for RF voltages $>2.5 \mathrm{~V}$. The tuning ratio corresponding to $2.5 \mathrm{~V}$ RF DC over actuation voltages of $100 \mathrm{~V}-200 \mathrm{~V}$ is $230 \%$. Figure 3-24 shows the capacitance curves for Design 2 at $0 \mathrm{~V}$ and 2.5V RF DC voltages. The effect of the parasitic capacitance from the substrate, pads and strips is not considered in the calculation of the tuning ratios for both Design land Design 2.

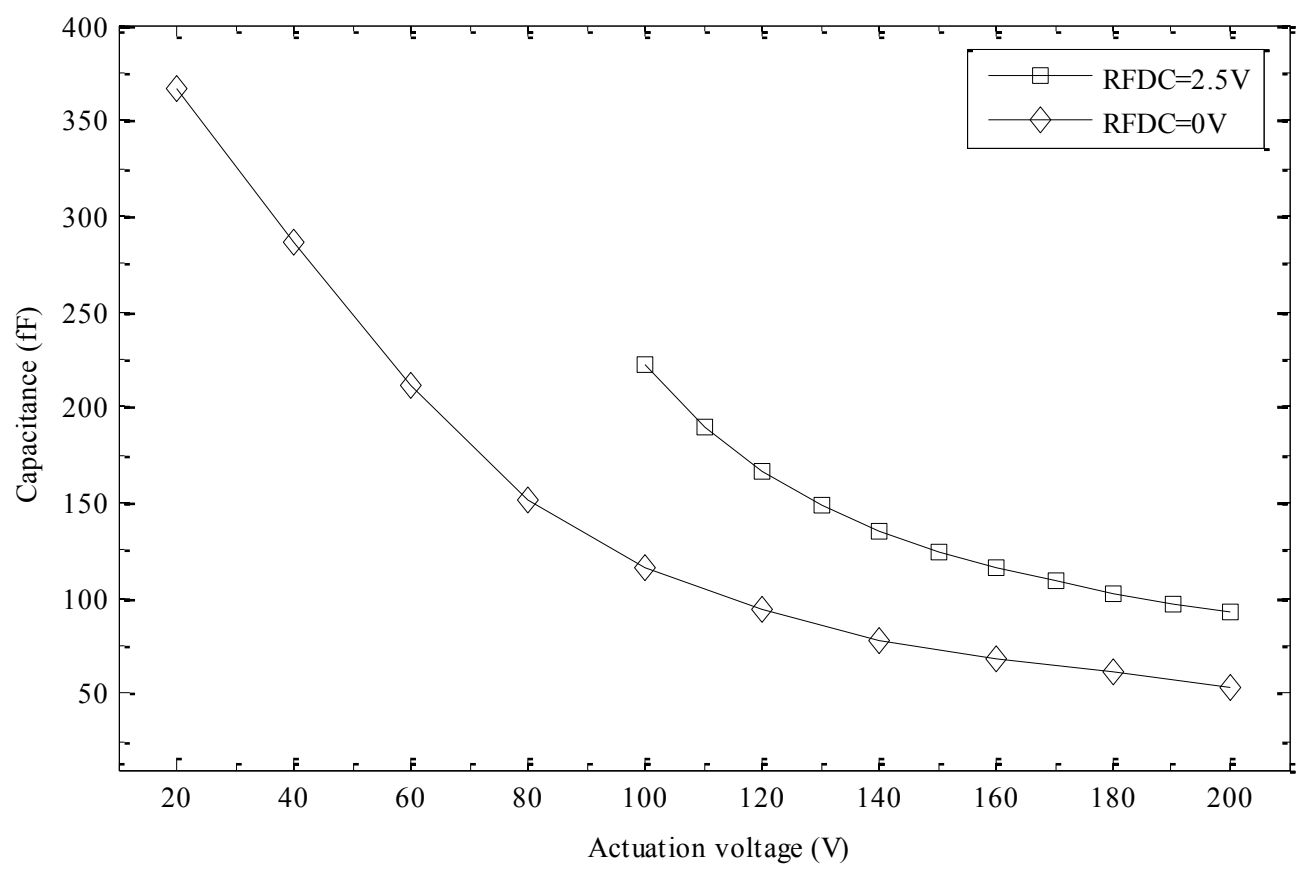

Figure 3-24 Simulated capacitance Vs actuation and RFDC equivalent voltages -Design 2

\subsubsection{Linearity factor and tuning ratios}

The linearity factor (LF), representing the coefficient of linear correlation between capacitance and voltage is calculated. The equation of linearity factor is [73]: 


$$
L F=\frac{n \sum_{i=1}^{n} C_{i} V_{i}-\sum_{i=1}^{n} C_{i} \sum_{i=1}^{n} V_{i}}{\sqrt{\left(n \sum_{i=1}^{n} C_{i}^{2}-\left(\sum_{i=1}^{n} C_{i}\right)^{2}\right)\left(n \sum_{i=1}^{i=n} V_{i}^{2}-\left(\sum_{i=1}^{n} V_{i}\right)^{2}\right)}}
$$

where $C_{i}$ is the calculated $i^{\text {th }}$ capacitance corresponding to the actuation voltage $V_{i}$. The linearity factor lies in the range $0 \leq|L F| \leq 1$. Using the data provided in Table 3-11 and Table 3-12 the linearity factor obtained for the parallel plate capacitor (Design 1) is $96.28 \%$. The linearity factor for inclined plate (Design 2) is $95.14 \%$.

\subsection{Prototypes and testing}

Sample prototypes of the tunable capacitors Design 1 and Design 2 are fabricated using the PolyMUMPs surface micromachining process. The experimental results are presented subsequently.

\subsubsection{Apparatus and equipment setup}

Figure 3-25 and Figure 3-26 show the equipments used for testing the tunable capacitors. Optical white light interferometer Zygo ${ }^{\circledR}$ new view $6 \mathrm{~K}$ is the microscope used for visual inspection and DC voltage tests. This microscope captures the images of a sample and enables to analyze the surface maps. The Metro-Pro application software is used to control the microscope's zoom in/out properties and to read the surface maps. Other instruments used to in this experiment are DC voltage source (Figure 3-25), high voltage amplifier and Wentworth DC probe manipulators with Tungsten DC probes. The entire setup for this experiment is shown in Figure 3-25 (a) and (b). The lens used in this experiment has magnification factor of two. Voltage feeder wires are connected to the DC probes through a $\mathrm{Z}$ shaped metal rod that is welded to the DC probe manipulators. The resolution of the microscope is $1.08 \mu \mathrm{m}$ and its field of view is $0.54 \mathrm{~mm} \mathrm{x}$ $0.54 \mathrm{~mm}$ when a lens of X20 with X1 zoom is used. 


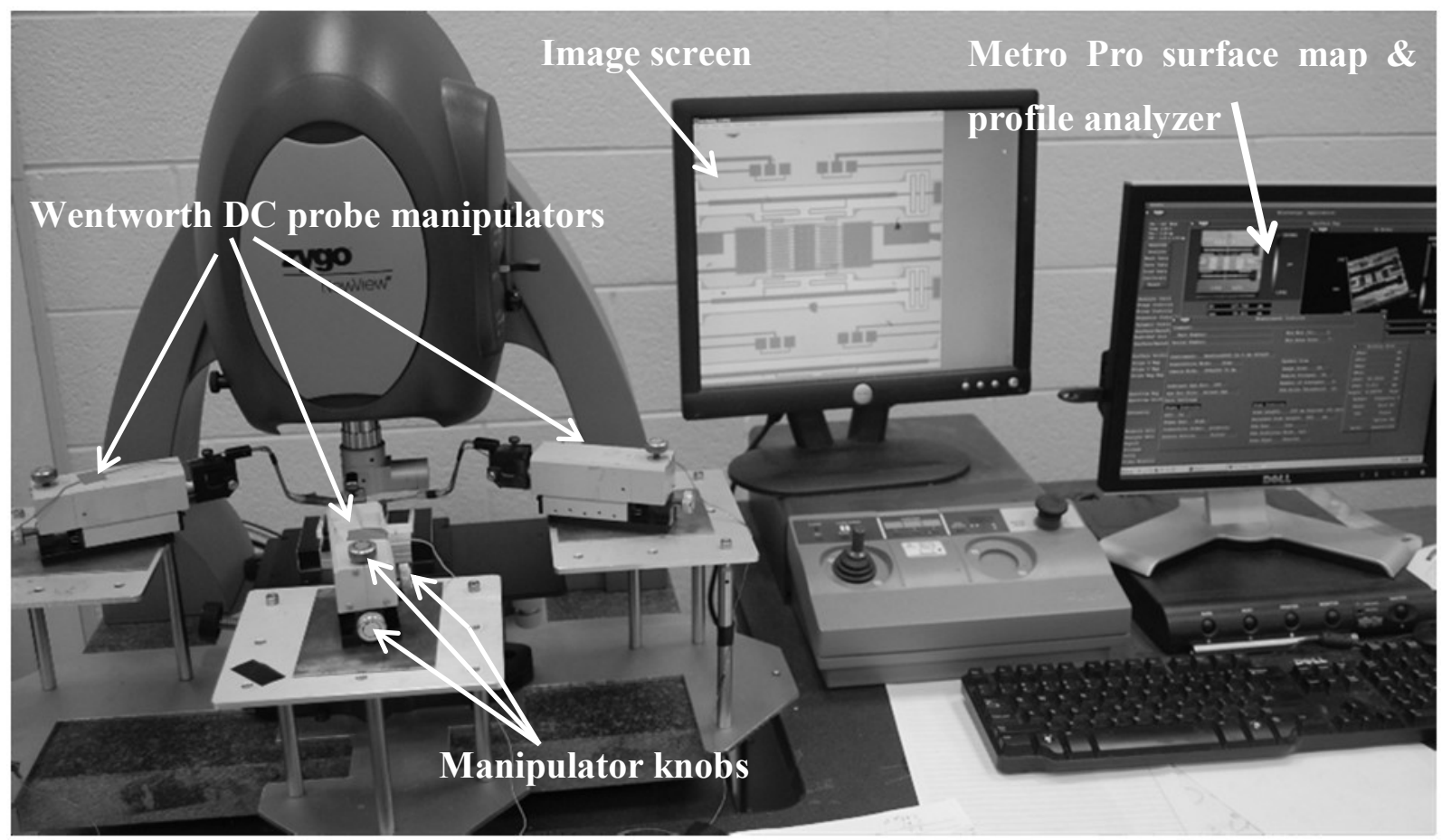

(a)

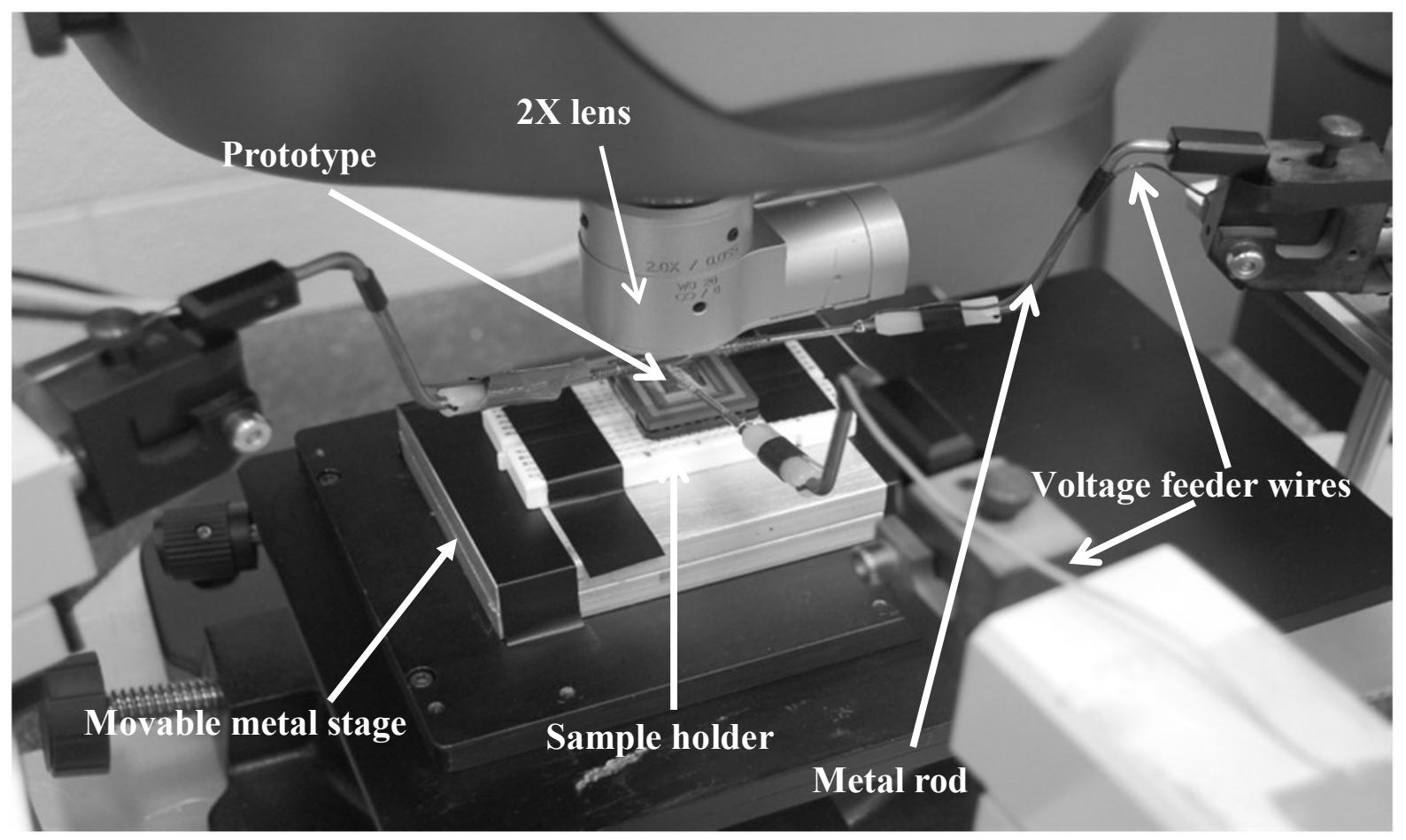

(b)

Figure 3-25 (a) Complete setup for DC test (b) Close view of probes and sample prototype 

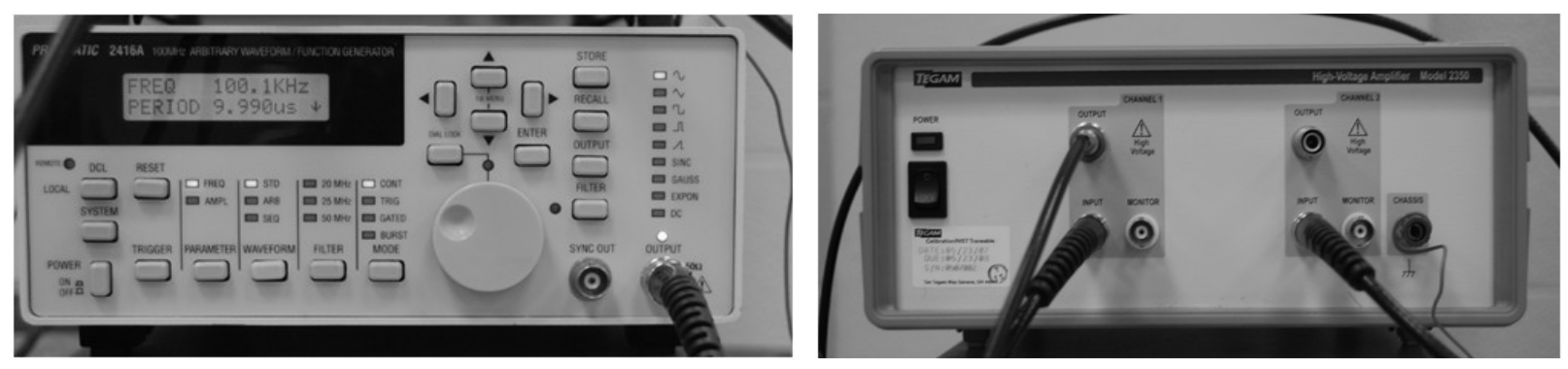

Figure 3-26 Voltage source and amplifier

\subsubsection{Experimental results}

In the experiment, profile measurements without actuation voltage and microscope inspection are conducted. The microscope inspections are used to verify whether the moving structures are free standing or the sacrificial layers are properly removed. The measurements refer to the height of the moving structures such as: the moving plate, the moving fingers and the supporting beams above the substrate. The vertical distance between the top surface of the moving plate and the top surface of nitride layer is measured on the profile analyzer. For all the measurements taken in this chapter the top surface of the nitride layer is chosen as a reference for measuring the heights. A scan length of $40 \mu \mathrm{m}$ and lens of $2 \mathrm{X}$ magnification is chosen for the measurement. The initial gap between the fixed and the moving plates of the capacitors is then obtained by subtracting the nominal thicknesses of the Poly 0 and the Poly 1 layers from the measured height. The nominal thickness values are used as the base for comparison whether the moving structures: the actuator fingers and the moving plate are released.

The measurement is read by drawing vertical or horizontal slice lines to extract points on the surface maps. The location through which the slice line passes is plotted on the surface profile. The heights of the structures can then be measured using the inspector axes. The inspector lines shown on the profile demarcate two boundaries and display the height difference or the gap between the boundaries. The inspector axes are manually adjustable on the profile plot. The images of Design 1 and Design 2 are shown in Figure 3-27. 


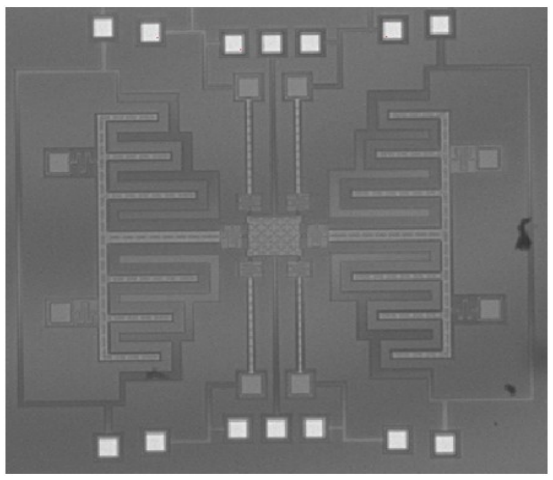

(a)

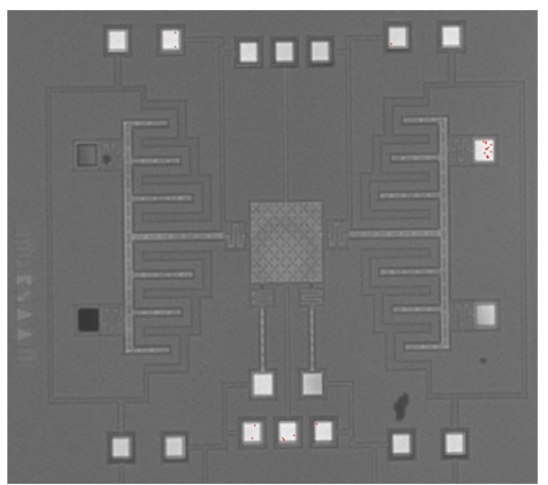

(b)

Figure 3-27 Images of capacitors: (a) Design 1 and (b) Design 2

\subsubsection{No-voltage measurements: Design 1}

The first step in the measurements is verifying whether the moving plates and the actuators are released after fabrication. The measurements without actuation voltages are referred to as novoltage measurements and are shown in Figure 3-28 and Figure 3-29. The measured height above the nitride layer, indicated by the slice line $A A$, is $4.63 \mu \mathrm{m}$. The measured thickness of Poly 0 exceeds $0.7 \mu \mathrm{m}$. Figure 3-28 (b) shows that the thickness of the Poly 0 is approximately $0.8 \mu \mathrm{m}$. Thus, the gap between the fixed electrode (Poly 0) and the moving plate of the capacitor found to be $1.83 \mu \mathrm{m}$ (nominal thickness $2 \mu \mathrm{m}$ ) based on the nominal thicknesses.

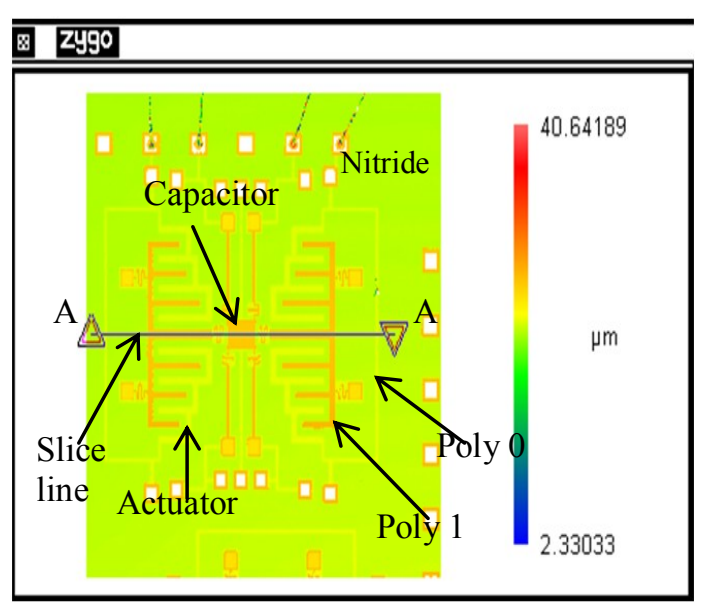

(a)

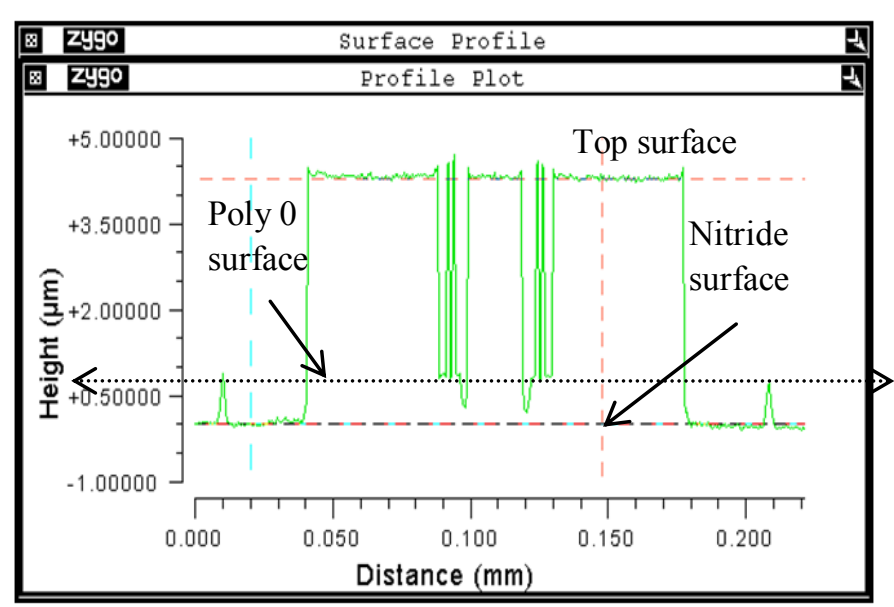

(b)

Figure 3-28 Moving plate measurement: no-voltage (a) Surface map and (b) Profile plot 

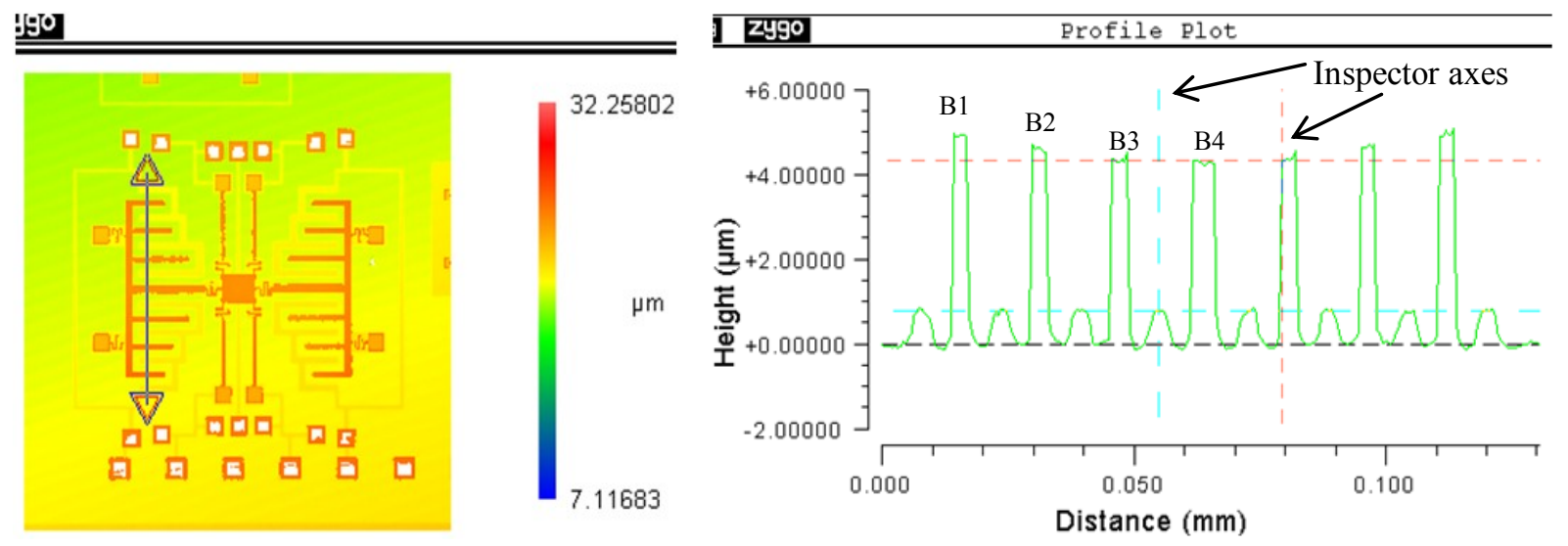

Figure 3-29 Actuator fingers measurement: no-voltage

In a similar way, additional measurements have been examined over randomly selected locations where the Poly 0 strips exist. The thickness of the strips in general is far from the range specified in [62]. The next measurements are taken to identify whether the actuator structures are released. Based on this measurement the moving plates are released i.e. air gap exists between Poly 0 and Poly 1 . The air gap has its height in the range of $0.75 \mu \mathrm{m}$ to $2.25 \mu \mathrm{m}$. The $0.75 \mu \mathrm{m}$ gap is due to the sagging effect of the plate and the moving fingers.

Figure 3-29 shows the heights of the actuator fingers above the fixed fingers. The shorter fingers have achieved larger heights compared with the longer fingers. The fingers B1, B2, B3 and B4 have heights of $2.1 \mu \mathrm{m}, 1.7 \mu \mathrm{m}, 1.5 \mu \mathrm{m}$ and $1.5 \mu \mathrm{m}$ respectively. The moving fingers are released and suspended freely above the Poly 0 .

\subsubsection{No-voltage measurements: Design 2}

The profiles of capacitor Design 2 are similarly inspected using the microscope. The measured heights of the moving plate of the capacitor and the actuators are shown in Figure 3-30 and 31. The moving plate is $1.4 \mu \mathrm{m}$ higher above the Poly 0 layer. The height is reduced due to the sagging effect caused by the total length the central beams, the springs and the large area of the moving plate. 


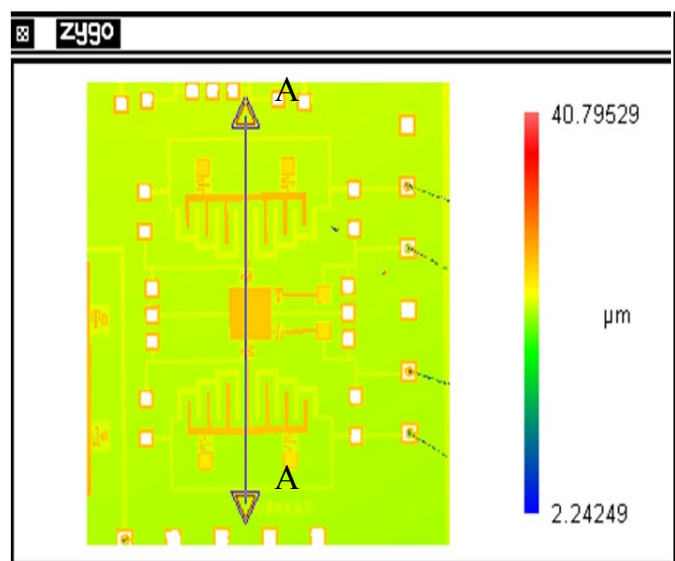

(a)

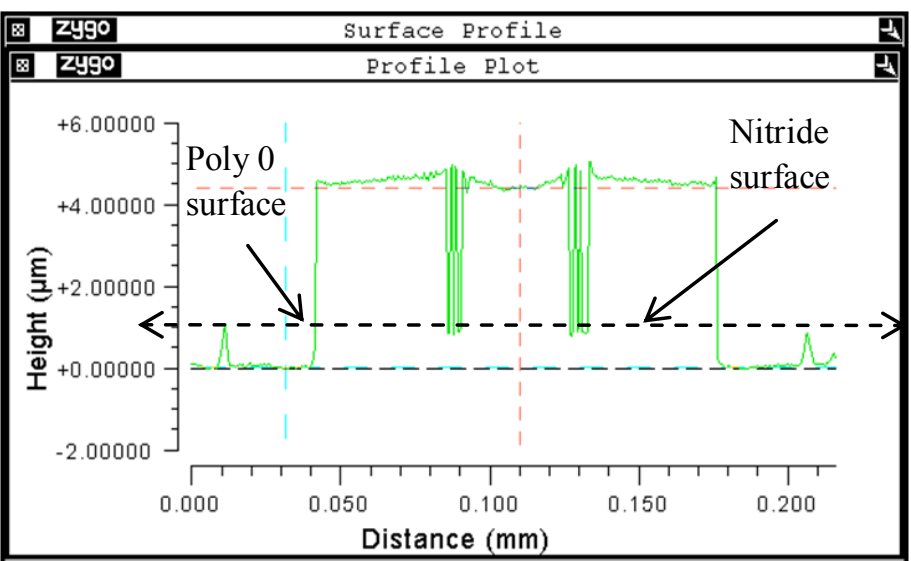

(b)

Figure 3-30 Design 2 (a) Surface map and (b) Profile plot
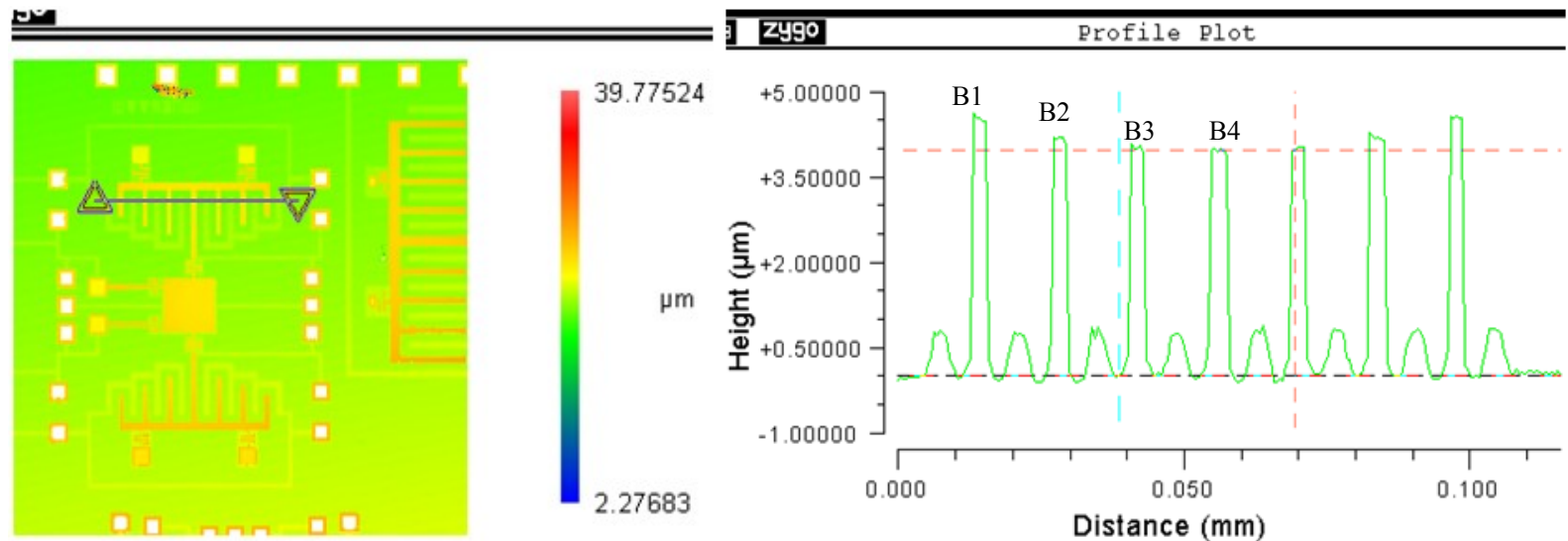

Figure 3-31 Design 2 Actuator (a) surface map and (b) profile plot

The actuators heights above the fixed fingers are also analyzed based on the profile plot shown in Figure 3-31. The gap between the aligned fingers B1, B2, B3 and B4 1.8 $\mu \mathrm{m}, 1.5 \mu \mathrm{m}, 1.2 \mu \mathrm{m}$ and $1.2 \mu \mathrm{m}$ respectively. Therefore, the moving plate and the actuators are released.

\subsection{Electromechanical tests: DC voltage test}

In the next two sub-sections electromechanical test results, analysis and observations are presented. By electromechanical performance tests, voltages are applied to the actuators to drive the moving plates of the capacitors and measure the resulting displacement. Voltage source PRAGMATIC $^{\circledR} 2416 \mathrm{~A}$ and TEGAM $^{\circledR}$ high-voltage amplifier 2350 (Figure 3-26) are used to supply the voltages. 


\subsubsection{DC voltage test-Design 1}

This part is focused on the measurements displacements of capacitor Design 1 under the presence of DC voltages. Voltages from $0 \mathrm{~V}$ to $150 \mathrm{~V}$ are applied with a $25 \mathrm{~V}$ step. The measured displacements of the moving plate at the applied actuation voltage are compared with the novoltage measurements to obtain the distance moving plate and the actuators reach. The measurement results of the moving plate at $25 \mathrm{~V}, 100 \mathrm{~V}$ and $150 \mathrm{~V}$ are illustrated in Figure 3-32 to Figure 3-34. Comparison between the profiles plotted in Figure 3-32 and Figure 3-28 show that the moving plate of the capacitor moved down by $1.05 \mu \mathrm{m}$ at $25 \mathrm{~V}$ i.e. the gap between the capacitor plates is $0.95 \mu \mathrm{m}$. As the voltage increased to $100 \mathrm{~V}$ it was difficult to pull up the moving plate even though the actuators are raised above the substrate by the increased electrostatic repulsive force. At this time the distance travelled by the plate is $1.3 \mu \mathrm{m}$, i.e. the gap is reduced to $0.7 \mu \mathrm{m}$ which is approximately equal to the heights of the dimples.

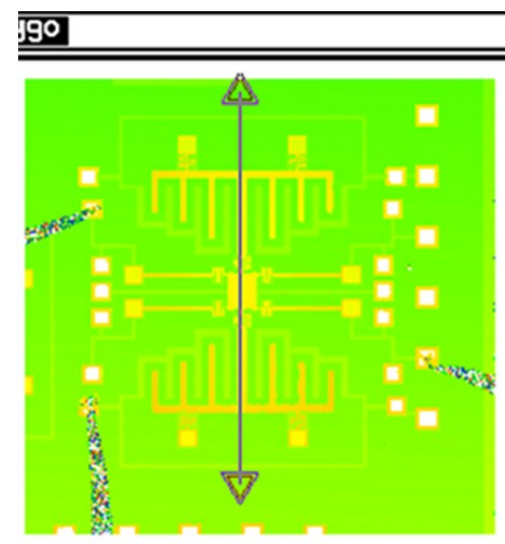

(a)

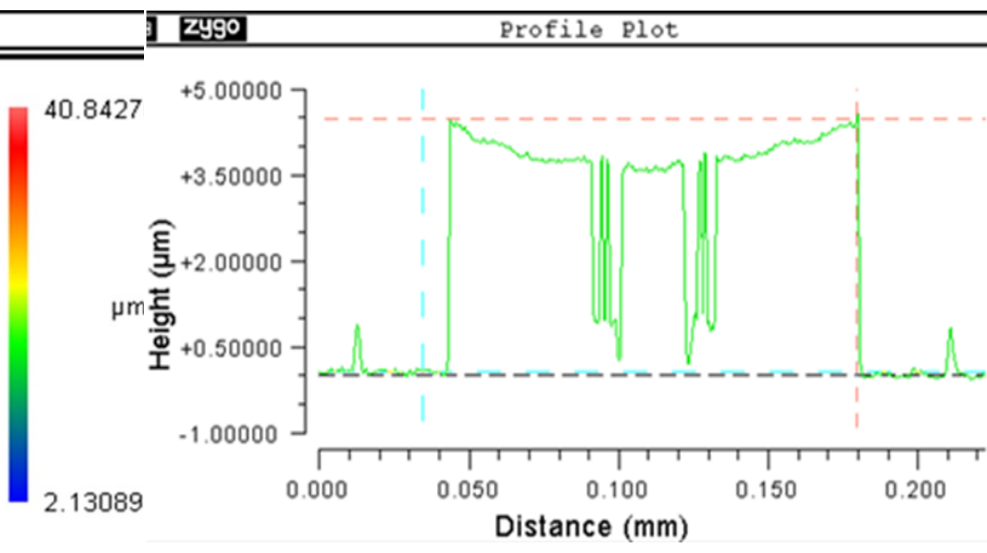

(b)

Figure 3-32 Design 1(a) Surface map and (b) Profile plot at 25V actuation 

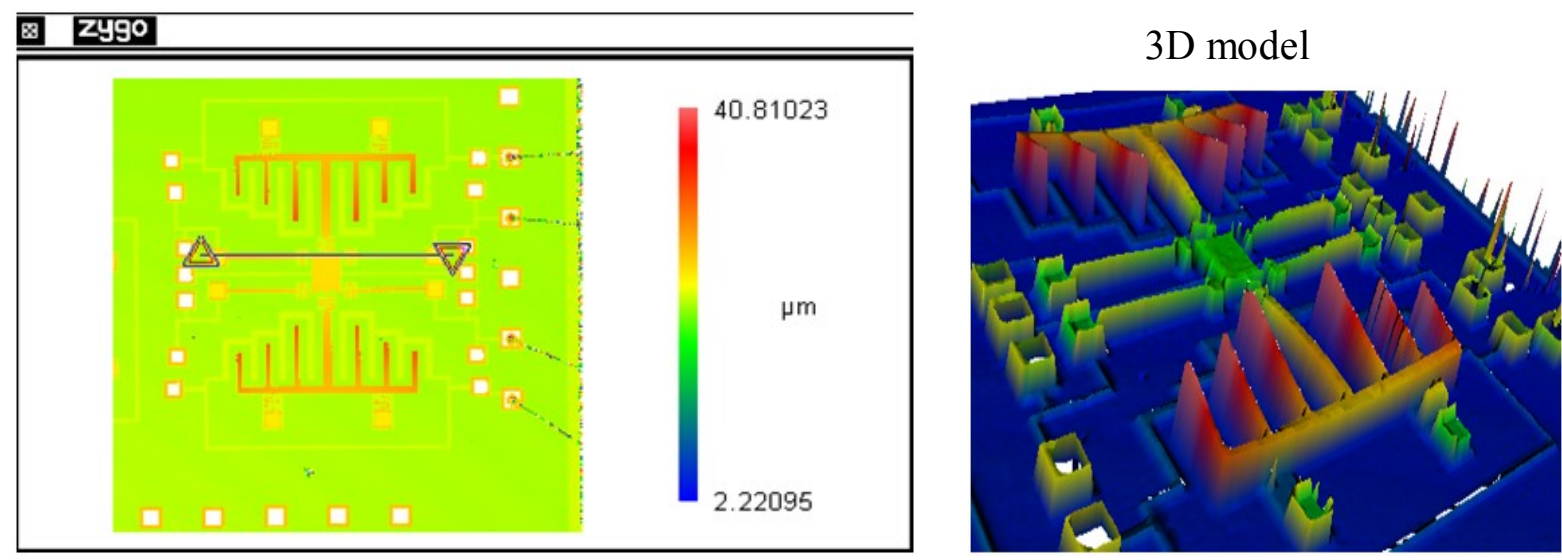

(a)

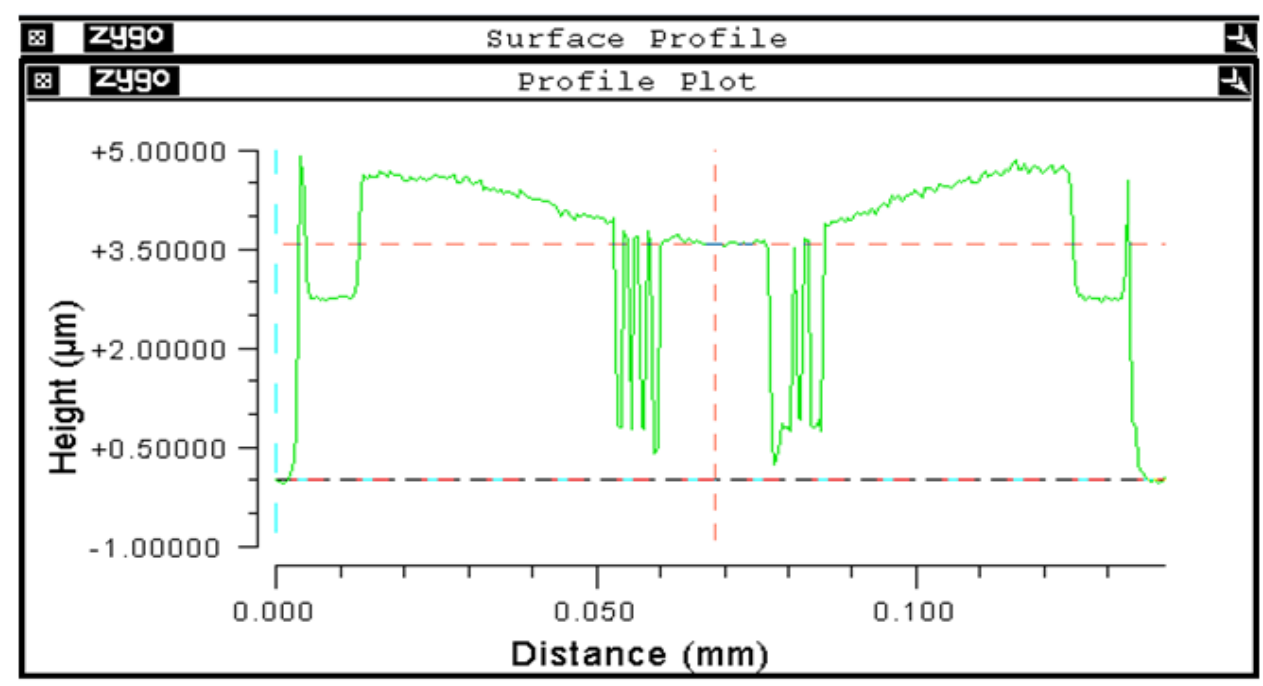

(b)

Figure 3-33 Design 1 (a) Surface map and (b) Profile plot at 100V actuation

Figure 3-34 shows the measured displacement of the moving plate of capacitor Design1 when $150 \mathrm{~V}$ is applied across the actuator. The measured distance between Poly 0 and Poly 1 is found to be $0.7 \mu \mathrm{m}$. From this measurement the moving plate travelled $1.3 \mu \mathrm{m}$ down. This indicates that the moving plate is pulled down towards the RF electrode by unknown electrostatic attraction force. 

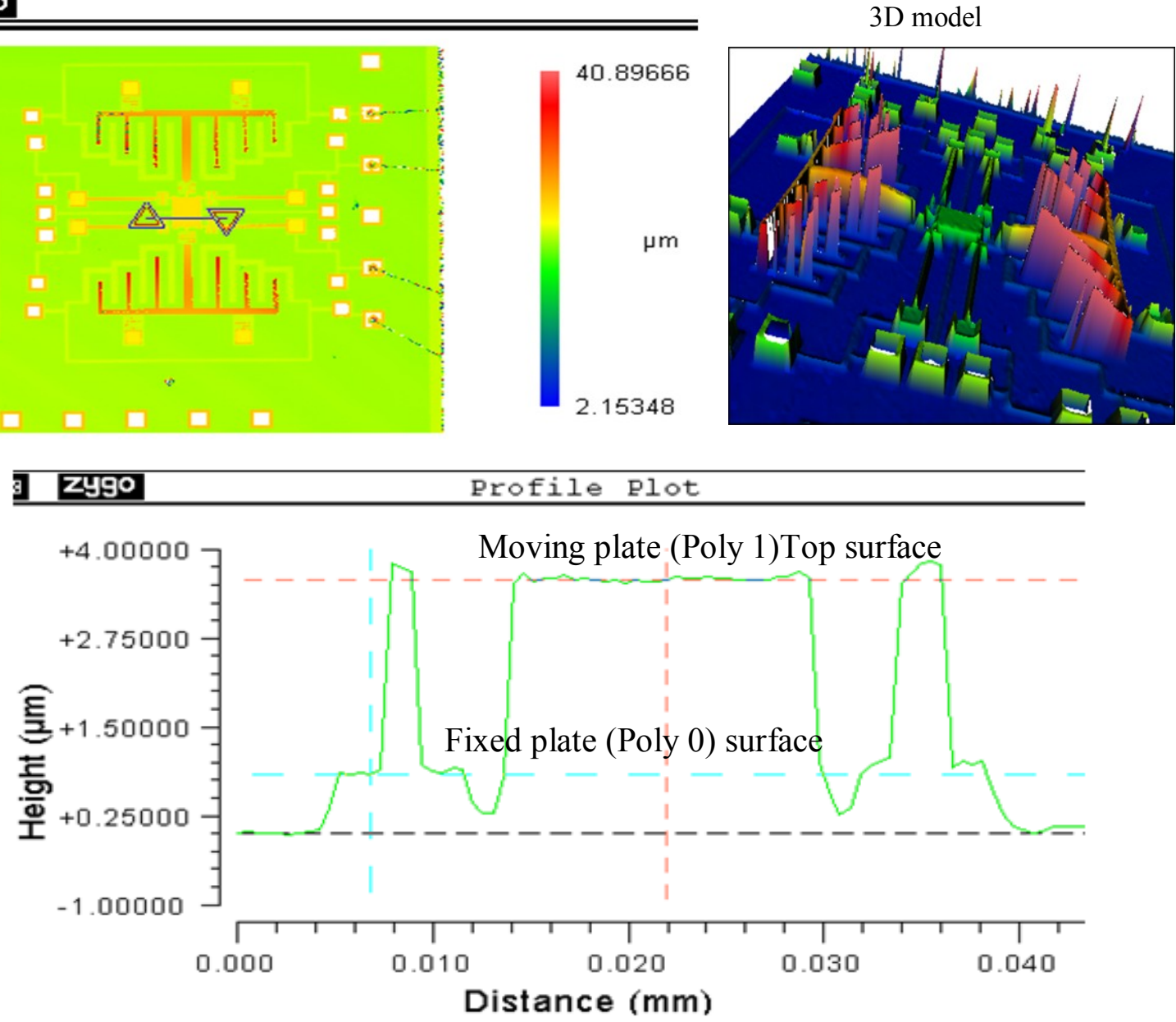

Figure 3-34 Plate displacement at 150V

However, the actuators showed upward displacement as shown in Figure 3-33 to Figure 3-35. The 3D models show that the moving plates are the lower elevation compared to the actuators. The graph in Figure 3-35 shows the measured heights of the actuator fingers at $150 \mathrm{~V}$. The fingers B1, B2, B3 and B4 obtained displacements of $13.5 \mu \mathrm{m}, 11 \mu \mathrm{m}, 10 \mu \mathrm{m}$ and $7.2 \mu \mathrm{m}$ along the slice line $A A$. 

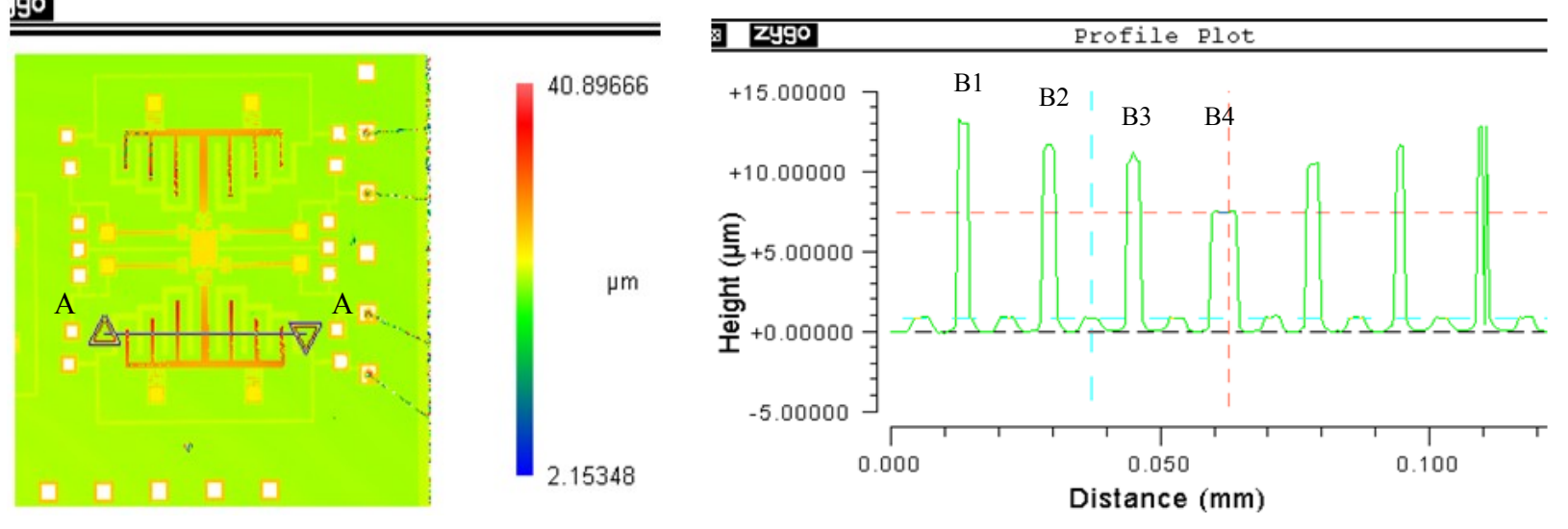

Figure 3-35 Actuator displacement at 150V

\subsubsection{DC voltage test-Design 2}

The DC voltage measurement for Design 2 has been done in a similar way shown in previous section. Figure 3-36 (a) and (b) show the surface map, the 3D model and the profile plots measured at $100 \mathrm{~V}$. As it was observed in Design 1, the moving plates moved down while the actuators are raised above the substrate. Figure 3-37 and 3-38 show the surface map of the moving plate and the fingers of the actuators at $125 \mathrm{~V}$. The actuators achieved larger displacement where as the moving plate remained attached to the substrate.
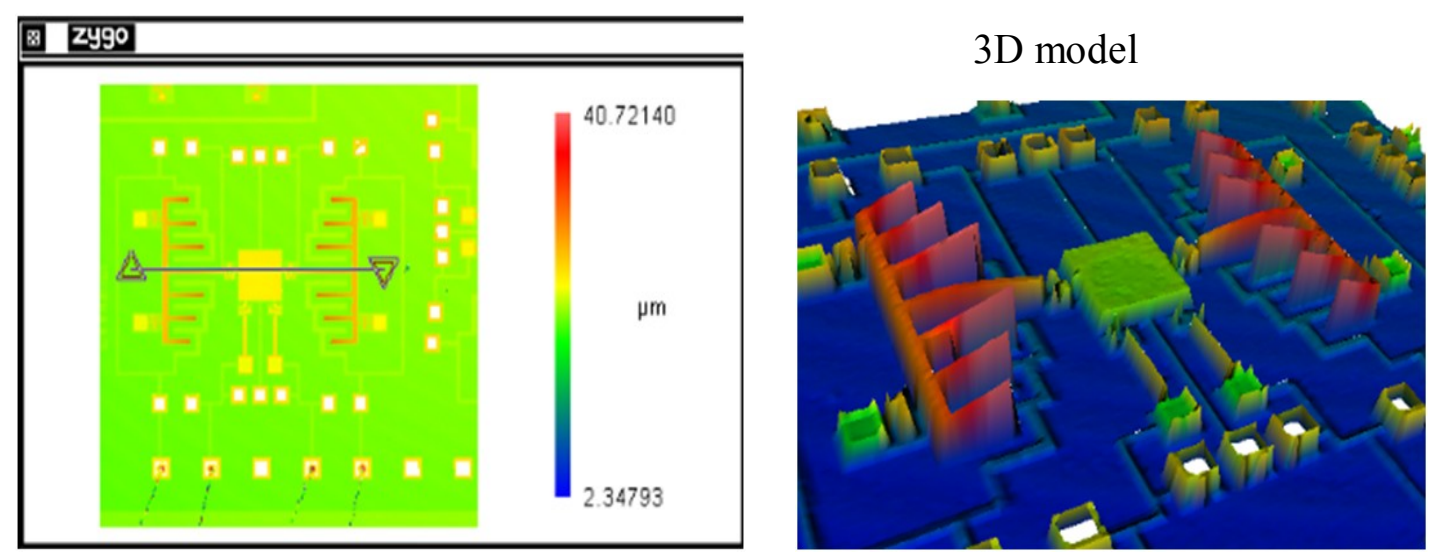

(a) 


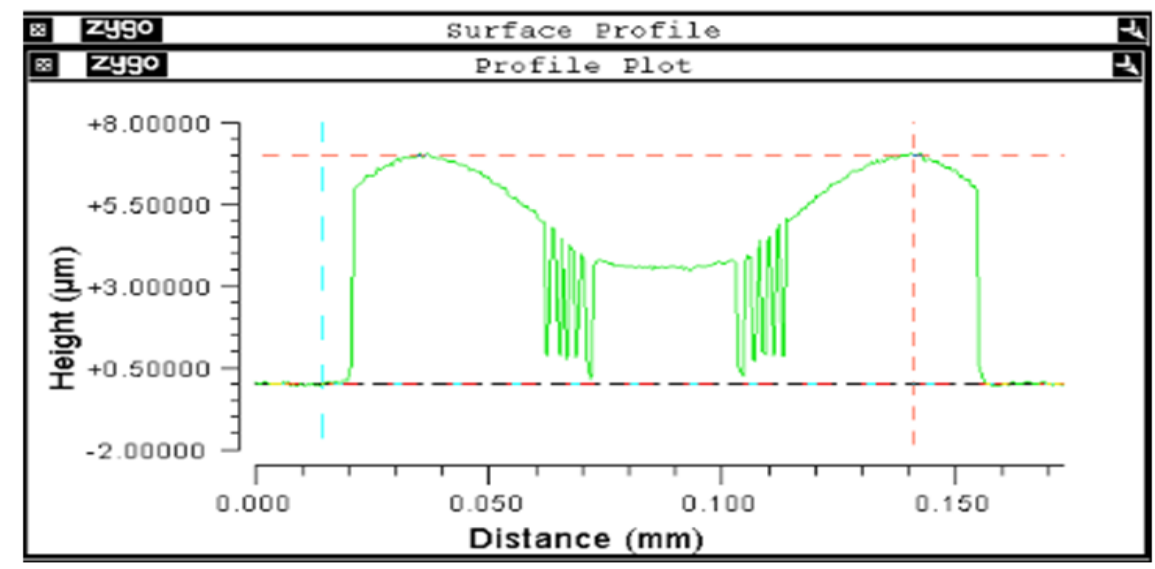

(b)

Figure 3-36 Design 2 (a) Surface map and (b) Profile plot at 100V actuation

\section{可o}

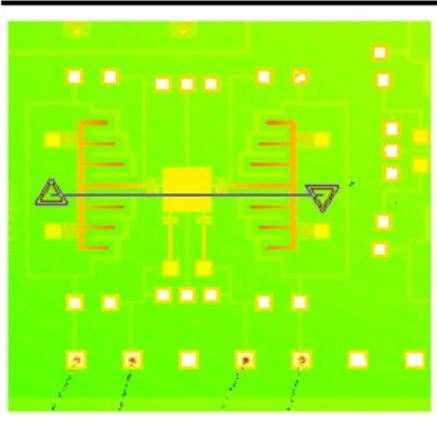

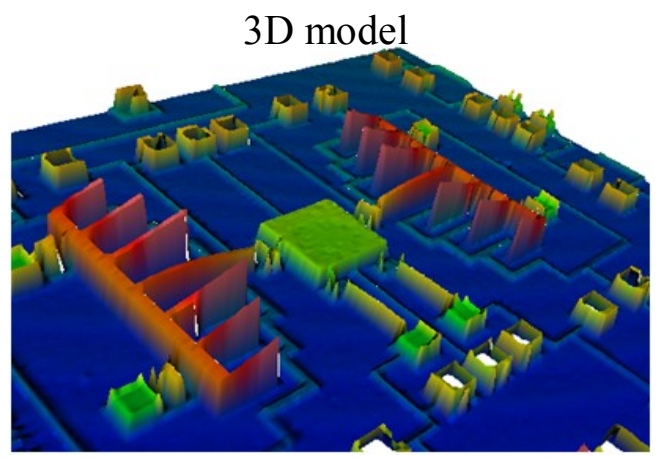

(a)

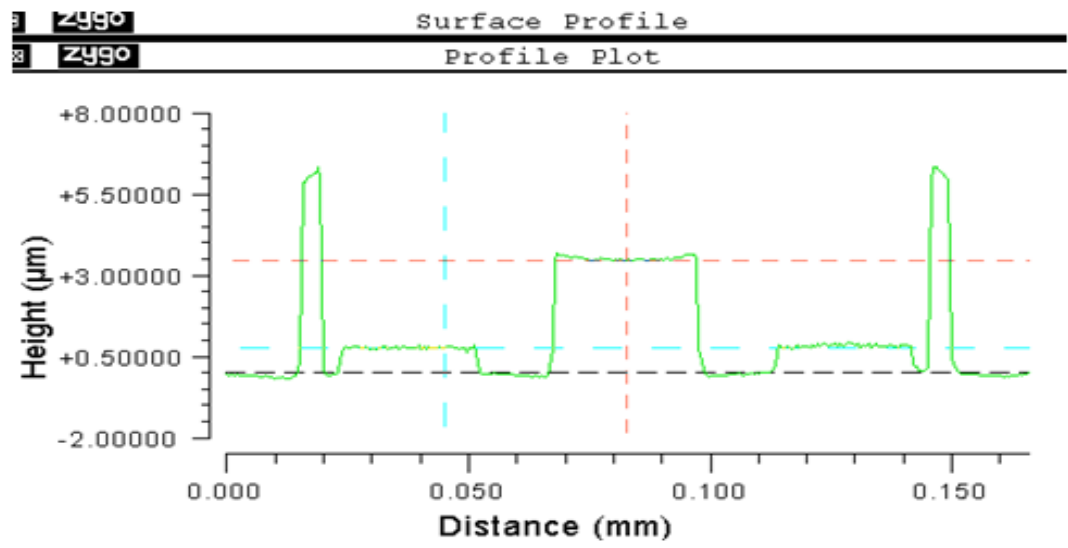

(b)

Figure 3-37 Measured displacement of the moving plate at 125V-Design 2 

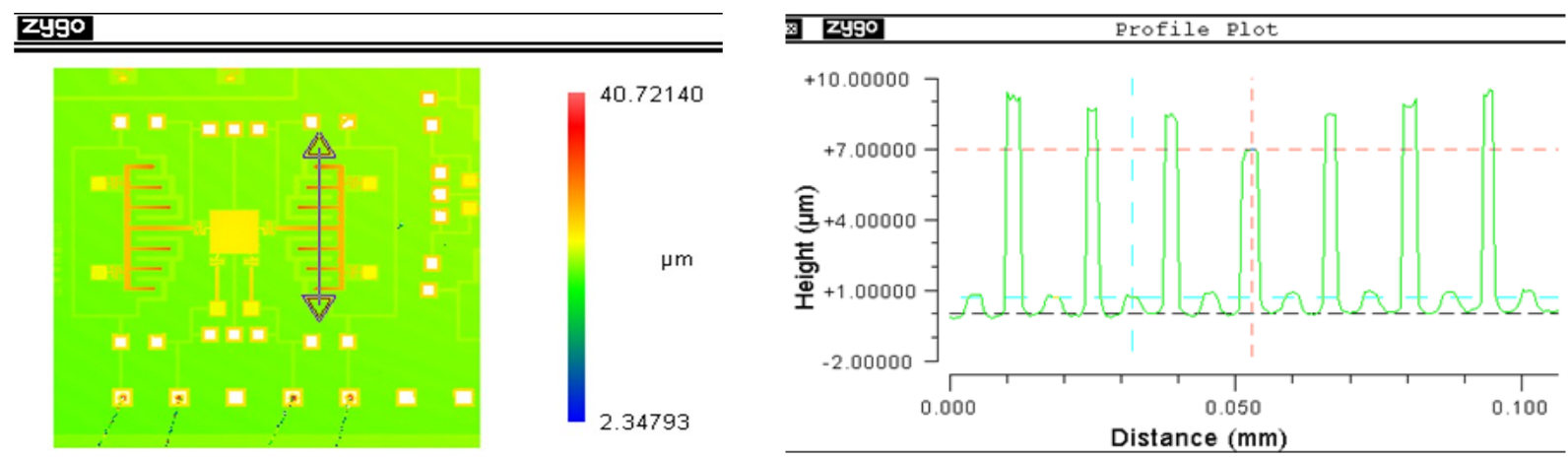

Figure 3-38 Actuator profiles at $125 \mathrm{~V}$

In conclusion, the results of the microscope inspections and measurements indicate that the moving structures are released. However, under voltage tests the measured heights of the moving plate are less than the measurements achieved under no-voltage measurements. It was proved that the moving plate moved towards the substrate. Contrary to this, the moving fingers of the actuator were pushed away from the substrate as result of the repulsive electrostatic force. After the actuation voltage is turned off and the DC probes are disconnected, the capacitor plate could not return to the original position i.e. the no-voltage measurements were not maintained. The final gap between the plates of the capacitor is found to be the heights of the dimples $(\sim 0.75 \mu \mathrm{m})$. The moving plate of the capacitor are attracted to the RF electrode and remained in permanent contact. Based on the analysis, an unknown static charge on the RF electrode of the capacitors generated electrostatic attraction force. The potential reasons for the presence of attraction force on the RF electrode of the capacitor could be charge in the silicon-nitride and the silicon substrate. The following section discusses electrostatic charging of the RF electrode using 2dimensional electrostatic simulations.

\subsection{Analysis of attraction force induced on the RF electrodes}

In the experimental result analysis it was observed that the moving plates of the capacitors were attracted down when voltages were applied to the actuators. The RF electrodes of the capacitors were neither connected to the DC terminals nor grounded. However, an unknown attraction electrostatic force was generated on the RF electrode to pull the moving plate down. The moving plates then failed to return to the original position after the voltages were cutoff. In order to prevent the short circuiting between the plates, the RF electrode and the substrate were grounded while the actuation voltages were supplied. However these techniques did not help to prevent the 
moving plate from moving down and bring the moving plate back to its original position. As a result it was necessary to study charging effect of the fixed electrode.

Electrostatic field simulation was carried out to visualize the electric field patterns along the moving plate, unaligned fingers and the RF electrode. The simulations were performed using Electro V63 2D simulator [74]. Figure 3-39 shows the cross section of the unaligned fingers and the capacitor plates. The material properties of the PolyMUMPs process were input into the database of Electro V63. The properties include the dielectric constants and the conductivities of the substrate, silicon nitride, Poly 0 and Poly 1 layers.

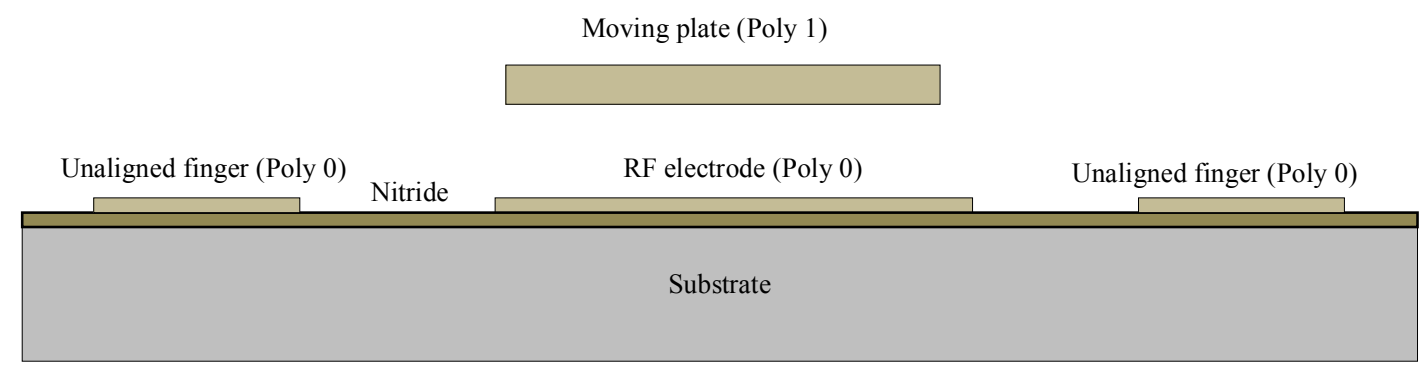

Figure 3-39 Cross-section view of the unaligned fingers and capacitor plates

The first simulation is focused on observing the direction of propagation of the electric field lines when actuation voltage and RF voltages are present across the actuator and the RF electrode of the capacitor.

In the second simulation, the RF electrode is fixed to a floating potential and voltages are applied across the actuator terminals. That means the RF electrode is not connected to the positive terminal or ground of the circuit. The electrostatic force and the induced voltage on the RF electrode are simulated when the potential of the electrode is floating. The simulated voltages are listed in Table 3-13. The simulation results (Figure 3-40) show an induced voltage on the RF electrodes of the capacitors.

Table 3-13 Simulated induced voltages and electrostatic forces on the RF electrodes

\begin{tabular}{lllllllll}
\hline Actuation voltage $(\mathrm{V})$ & 25 & 50 & 75 & 100 & 125 & 150 & 175 & 200 \\
Induced voltage $(\mathrm{V})$ & 1.16 & 2.32 & 3.46 & 4.64 & 5.8 & 6.97 & 8.13 & 9.3 \\
Force $(\mathrm{mN} / \mu \mathrm{m})$ & 0.2 & 0.8 & 1.8 & 3.2 & 5.0 & 7.2 & 9.8 & 12.8 \\
\hline
\end{tabular}




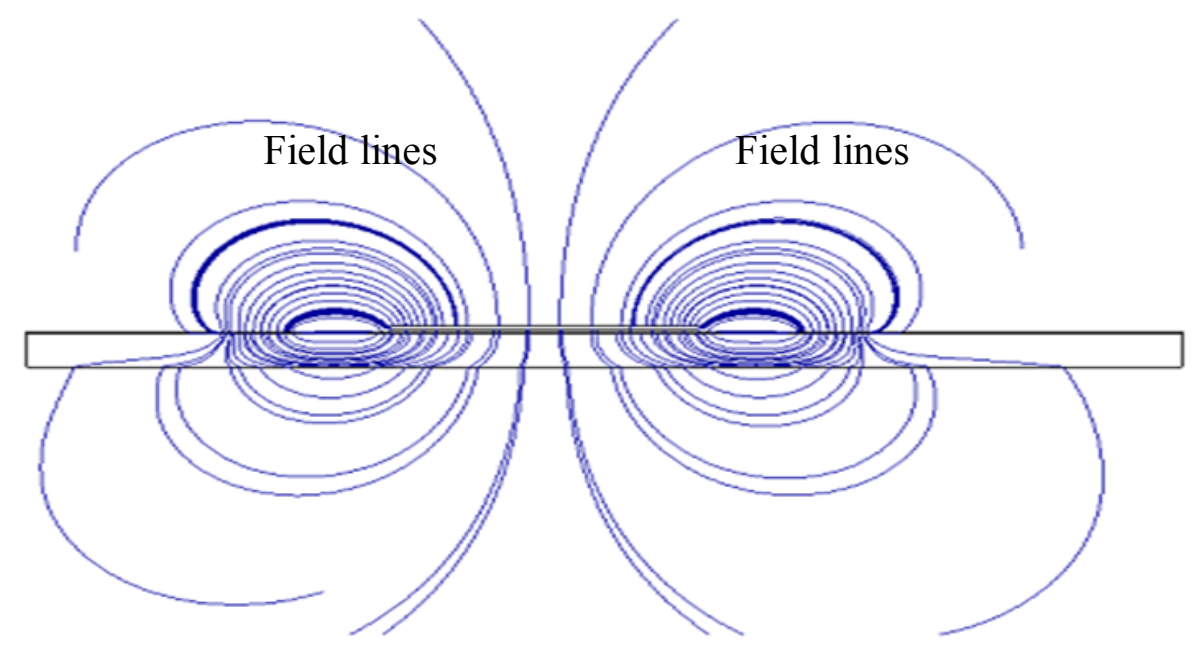

Figure 3-40 Field stream lines at 25V-wtih RF electrode floating

Attractive electrostatic forces could arise in the substrate because of the charge trapping ability of silicon nitride layer [76]. In the PolyMUMPs process charges may build near the Poly $0-\mathrm{Si}_{3} \mathrm{~N}_{4}$ interfaces. Stresses, defects, crystallography and ionizing beams such as electrons are likely to increase the charge accumulation in the Poly $0-\mathrm{Si}_{3} \mathrm{~N}_{4}$ interface.

Two methods are proposed here to reduce the induced voltage from the neighboring actuator electrodes. The method is using a loop of Poly 0 strip that is connected to the ground (0V) terminal of the capacitors. Simulation results using electro software show that the voltage can be reduced to $\sim 2 \mathrm{~V}$.

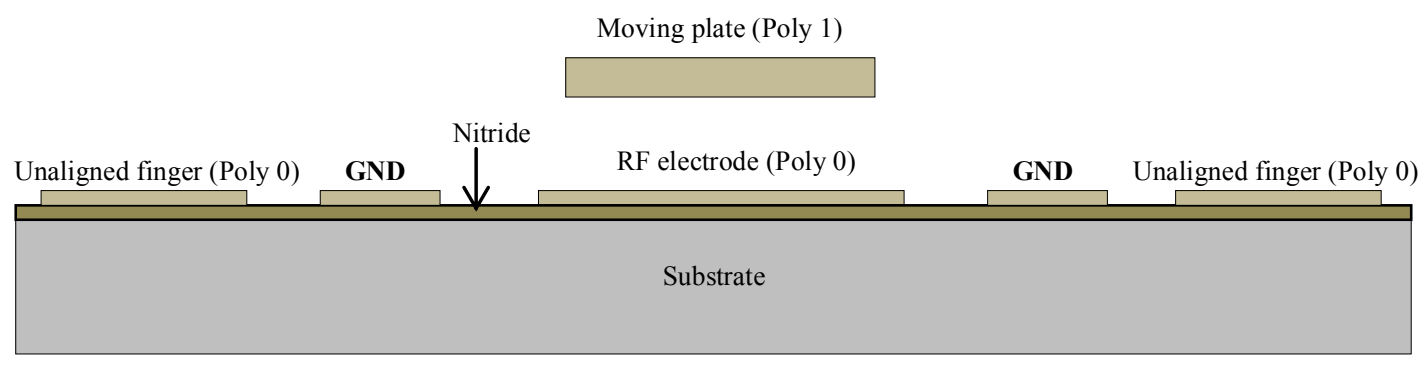

Figure 3-41 Proposed techniques with ground loop around the RF electrode

The Poly 0 that serves as loop is labeled, as shown in Figure 3-41, serves to suppress the electric fields coming from the nearby actuator electrodes. The simulated induced voltages are listed in Table 3-14. The width of the GND strip used is $40 \mu \mathrm{m}$. When the GND strip is placed farther away from the RF electrode the induced voltage becomes low. 
Table 3-14 Simulated induced voltages and electrostatic forces on the RF electrodes

\begin{tabular}{l|l|llllllll}
\hline \multicolumn{2}{l|}{ Actuation voltage (V) } & 25 & 50 & 75 & 100 & 125 & 150 & 175 & 200 \\
\hline Induced voltage (V) & $10 \mu \mathrm{m}$ & 0.252 & 0.501 & 0.757 & 1.004 & 1.265 & 1.500 & 1.765 & 2.005 \\
when the GND Poly 0 & $20 \mu \mathrm{m}$ & 0.236 & 0.471 & 0.71 & 0.945 & 1.178 & 1.418 & 1.655 & 1.89 \\
strip is located at & $40 \mu \mathrm{m}$ & 0.204 & 0.409 & 0.613 & 0.817 & 1.03 & 1.227 & 1.413 & 1.634 \\
\hline
\end{tabular}

The second method is covering the silicon nitride layer by a ground electrode to completely suppress the charges that propagate from it. This technique was used to suppress the fields from the nitride layer of the electrostatic comb-drive actuator developed in [80].

\subsection{Elimination of RF short-circuiting}

The RF signal induces DC voltage that pulls the moving plate and causes short circuiting. As a result, the moving plate cannot return to the original position if the RF is signal power is high. This situation may be caused by the pull-in effect of the RF signal. In the capacitor designs presented previously, high actuation voltage must be present to ensure the plates will not contact. However, high actuation voltages could not necessarily keep the moving plate away from the fixed electrode all the time. Attractive electrostatic forces are large compared with the repulsive electrostatic forces of the actuators used to drive the capacitors. Preventive methods are required to eliminate the pull-in effects of the RF signal.

Dielectric thin films have been widely used to minimize the pull-in effect [75], [79] that occurs from RF voltage. Dimple structures have also been suggested as a safety structure to minimize electrical between the plates of a capacitive switch [79]. A technique suggested in this thesis is using holes on the RF electrode of the capacitor. The holes allow the landing of the moving plate on the nitride through the dimples. The holes are etched under each dimple as shown in Figure 3-42. The nominal depth of the dimples is $0.75 \mu \mathrm{m}$ which is larger than the thickness of the Poly $0(0.5 \mu \mathrm{m})$. Hence, the air gap between the plates of the capacitor becomes $0.25 \mu \mathrm{m}$ when the dimples touch the nitride layer. The cross-section view representation of the capacitor with dimples only is shown in Figure 3-42(a). In this case once the capacitor plates get contact the moving plate does not bounce to the original position. However, the presence of holes under the dimple, Figure 3-42(b), avoids the contact between the plates of the capacitor. Therefore, the pull-in effect of the RF voltage can be eliminated. 


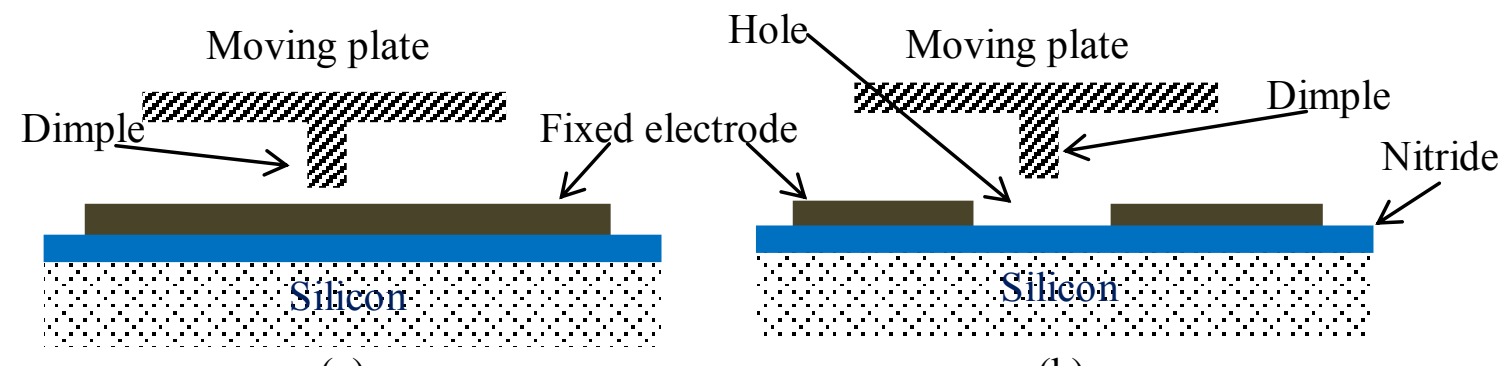

(a)

(b)

Figure 3-42 Hole and dimple on capacitor plates

\subsection{Chapter summary}

Gap increasing tunable capacitors with high tuning ratio and linearity have been designed, simulated and the results were analyzed in the chapter. Repulsive electrostatic actuation principle was used to drive the capacitors. The moving plates of the capacitors follow translational and rotational motions. The PolyMUMPs micro-fabrication process was used to fabricate the capacitors. CoventorWare finite element analysis software package was used to simulate capacitances, displacements and study capacitance-voltage relationships. The electromechanical simulation involving the effect of the RF power on the tuning ratio and the linearity was also analyzed. The RF power causes attractive electrostatic force that opposes the repulsive electrostatic force of the actuators. Electrostatic attractive force is stronger than repulsive electrostatic force for a given area. Hence, small values of change in the DC equivalent of the RF power cause significant reduction in the travel range of the moving plates of the capacitor. As a result, the tuning ratio of the capacitors is reduced considerably for high RF powers. Simulations conducted without RF power show the capacitor have maximum upward displacement of the $9.9 \mu \mathrm{m}$ (Design 1) and $14 \mu \mathrm{m}$ for $200 \mathrm{~V}$. However, when a $4 \mathrm{~V}$ RF DC equivalent is applied the maximum displacement of the capacitor reduces to $6.9 \mu \mathrm{m}$ for Design 1-transtaltion type capacitor.

Analytical expressions have been derived to calculate the capacitance at any voltage. The designs achieved high tuning ratios under RF DC equivalent. High capacitance-voltage linearity were obtained by using the electrostatic repulsive actuator as a driving unit of parallel plate tunable capacitor. Pull-in effect, which is common to electrostatic parallel plate actuators, is minimized. 


\section{A Novel Vertical Comb-Drive Actuator Using a Single Layer of MetalMUMPs Microfabrication Process}

\subsection{Introduction}

This chapter presents the design, working principle and fabrication of a novel electrostatic actuator which is applied to the design of RF MEMS tunable capacitors. MetalMUMPs microfabrication process has thick nickel layer which can be used to fabricate tunable RF MEMS components such as tunable inductors, switches and capacitors. Vertical comb drive actuators have found wide applications such as in the design of tunable capacitors, driving unit of deformable micro-mirrors for vision science, optical switch, optical attenuators, scanning mirrors and micro-accelerators. Vertical comb drive actuators require two structural layers to fabricate vertically separated fixed fingers and moving fingers. Fabrication of such actuator needs complex fabrication process, and is pricey.

In this thesis a new method is proposed to obtain vertically separated fixed fingers and moving fingers of a vertical comb drive from a single structural layer. The method exploits residual stress gradients along the thickness of a single layer to obtain two vertically separated fingers.

The MetalMUMPs micro-fabrication process provides thick nickel layer coated with a gold film. The combination of the nickel and the gold form a single layer known as metal of the MetalMUMPs process. A beam fabricated using this process is subject to residual stress gradient that cause bending during the fabrication process. A curve up beam is devised to bend upwards due to stress gradients. Comb fingers connected to the opposite sides of a plate are attached to the middle of the curve up beams through serpentine springs. When the curve up beam bends it lift up the moving comb fingers above the substrate and the fixed fingers.

On the other hand, the fixed fingers remain attached to the substrate through anchors. Therefore, two sets of structures can be formed from the metal layer, one moving and the other fixed. The actuator's pre fabrication deformations are analyzed using the CoventorWare simulations. The fabricated actuators are tested to verify the design and working principle. Finally, the results of the experiments are compared with the simulated results. 


\subsection{Residual stresses and stress gradients in micro films}

Stress gradient in micro films develop from fabrication processes [81] and change the behavior of the films. The stress gradients are internal and act along the thickness of the films to create contraction or expansion. The stress gradient deformations are typically observable on released thin film structures. According to Kuo-Shen Chen, [82] residual stress gradients depend on properties such as mechanical, thermal, chemical of materials, substrates boundary conditions and processing parameters during fabrication processes. Thin films also experience deformation as a result of residual stress gradient when sacrificial layers or substrates are etched away [92]. Residual stresses can also be governed by the deposition parameters, layer interactions with substrate, and thickness of the film [83-85]. Also stresses could develop from effects such as difference in thermal expansion coefficients, re-crystallizations, phase transformations and diffusion of impurities in and out of the films. The deformation caused by the residual stress gradient is analyzed for cantilever type beam and clamped-clamped type beams [86-101].

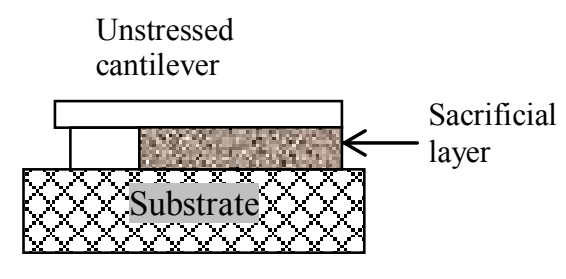

(a) Pre-stressed beam

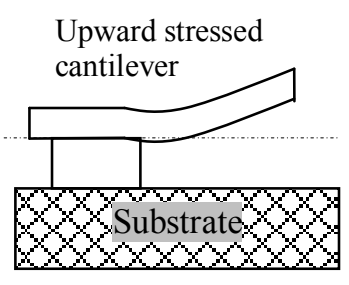

(b) Stressed beam 1

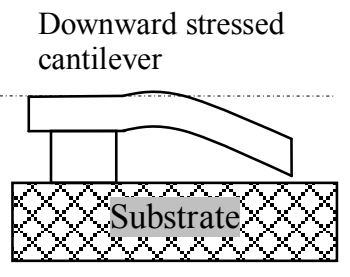

(c) Stressed beam 2

Figure 4-1 Types of stresses on a cantilever beam

Figure 4-1 shows the deformations on a MEMS cantilever beam due stress gradients. Residual stresses exist on the beams after the sacrificial layer is removed to deform the beams. The mean stresses elongate or shrink the beams and act in-plane. The stress gradients cause upward or downward bending of the beams. For example, positive stress gradients curve cantilever films upward (Figure 4-1(a)); whereas negative gradients curve the beams downward (Figure 4-1(b)) [82]. The stress gradients cause an out of plane deflection of beams.

Methods have been reported to determine the residual stress gradient of thin metal films [88-95]. The method used to determine the residual stress gradient is to measure the radius of curvature of the deformed beams [86-92]. Micro fabricated cantilever beams have been used to characterize tensile residual stress gradients [92-99]. Cantilever beams which are subject to compressive stress bend downward. However, it has been found that cantilever beams are not suitable to 
determine compressive residual stress gradients. Trenches or holes in the substrate are necessary for cantilever type beams to determine the bending caused by the gradient stresses $[99,100]$.

\subsection{Residual stresses in MetalMUMPs fabrication process}

MetalMUMPs process has an electroplated nickel $[101,103]$ which was used for fabricating micro mechanical capacitors [104], inductors [105, 106] and switches [107-109]. The residual stress deformation was observed on the plate of a tunable capacitor fabricated in [104]. S. He et al demonstrated the bending effect of the residual stress gradient [101] using beams made of MetalMUMPs metal layer. The stress gradient was found to be compressive and along the thickness of the nickel. Simulations conducted in this chapter showed that the stress gradient causes very long clamped-clamped beams to curve upward. The upper surface of the metal film expands more compared with the bottom surface. The beams fabricated thus have arch or dome shapes due to the expansion and contraction of the surfaces of the nickel. The length of the curved beams after fabrication is greater than the length of the beam before deformation. At final step of the release process, potassium hydroxide $(\mathrm{KOH})$ silicon etch is used to form a trench in the silicon substrate. It is expected that thermal gradients build up during the release of the sacrificial layer and silicon etching step to cause the buckling of the beams. Negative residual stress gradient that varies along the thickness was determined in [101]. The residual stress gradient varies along the thickness of the nickel. The patterns of deformations the beams attain after fabrication are illustrated in Figures 4-2 and 4-3 for clamped-clamped and cantilever beams, respectively. The clamped-clamped beams curve upward whereas the cantilever beams bend downward towards the substrate.

CoventorWare simulations are used in to analyze the deformation of clamped-clamped beams. The key parameters that determine the deformation are the stress gradient, the Young's modulus and the geometric parameters. It was found that as the stress gradients increase, deflection of the beams is larger. Very long beams yield higher deflection in the middle of the beam. 


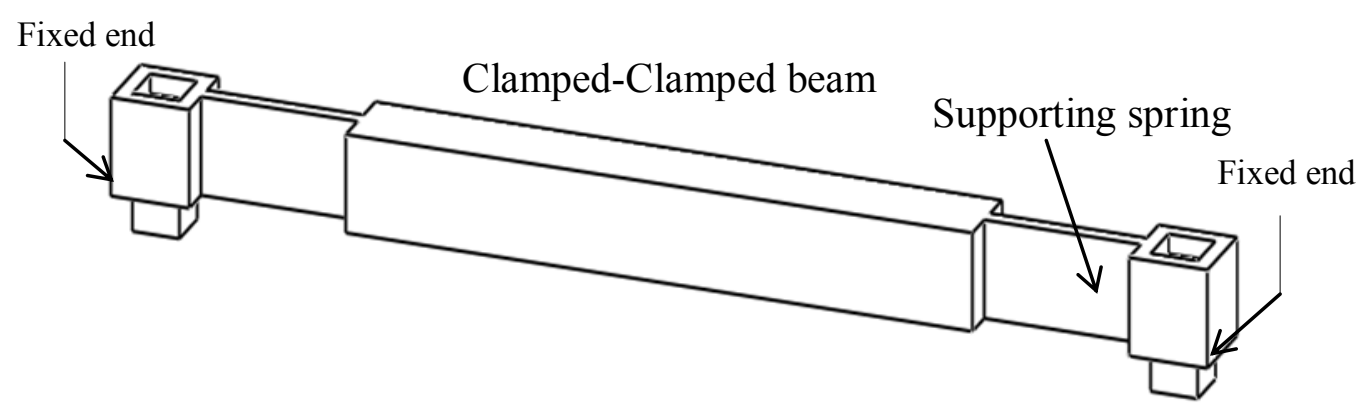

(a)

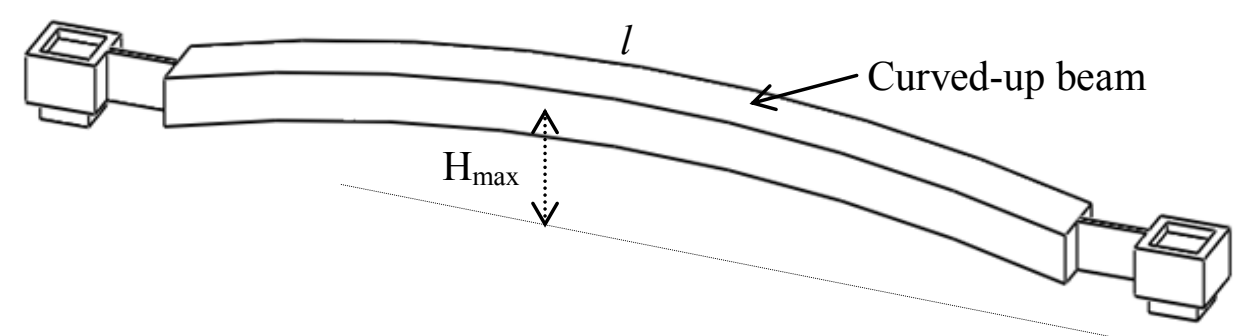

(b)

Figure 4-2 Residual stress gradient deformed clamped-clamped beam

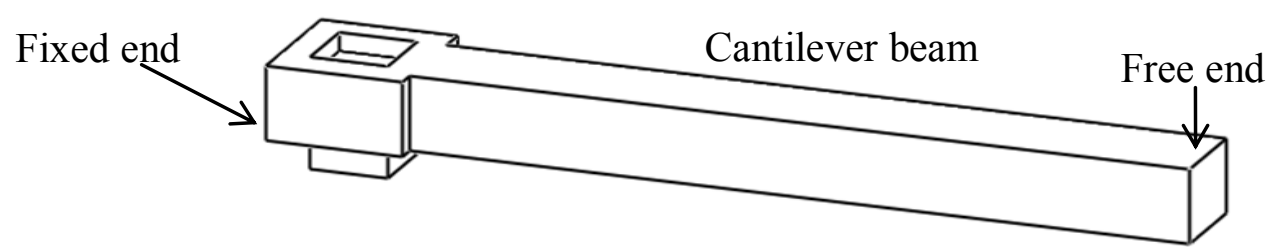

(a)

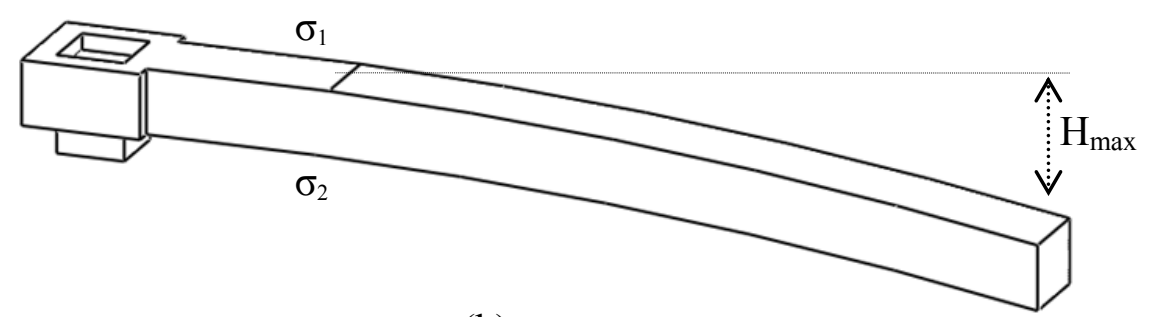

(b)

Figure 4-3 Residual stress gradient deformed cantilever beam 


\subsection{Micro-fabrication process-MetalMUMPs process}

The Metal Multi-User MEMS Process, MetalMUMPs, [81, 103] has nickel metal of nominal thickness $20 \mu \mathrm{m}$ and a coating gold of $0.5 \mu \mathrm{m}$. The MetalMUMPs nickel metal has comparatively attractive properties such as good electric conductivity and low resistivity due to its thickness. This process can be used to fabricate high quality factor RF MEMS components such as; tunable capacitors and inductors.

\subsubsection{Fabrication process steps}

The fabrication process starts with an npn-100 a silicon substrate followed by a thin oxide layer of height $2 \mu \mathrm{m}$. This oxide layer is used to define a trench hole in the silicon substrate. Then first silicon nitride (Nitride 1) of thickness $0.35 \mu \mathrm{m}$ is deposited using low-pressure chemical vapor deposition (LPCVD). A poly-silicon $(0.7 \mu \mathrm{m})$ layer is printed for signal routing over (Nitride 1). The second nitride (Nitride 2), 0.35 $\mu \mathrm{m}$ thick, is now deposited over the Polysilicon layer. A sacrificial layer of $1.1 \mu \mathrm{m}$ silicon dioxide is deposited on top of second nitride layer using LPCVD process. This layer forms the sacrificial layer which is etched away at the end of the fabrication process to release the structural layer.

The nickel film is deposited on a thin film of copper $(\mathrm{Cu})$ covered with a thin titanium (Ti) layer. This layer forms the plating base of the metal. The mask level for the metal is patterned using a thick resist which forms a stencil for the metal layer. The nickel film, with nominal thickness of $20 \mu \mathrm{m}$ is electro plated in to the patterned resist. A gold layer of $0.5 \mu \mathrm{m}$ is immediately electroplated on the top of the nickel. The gold layer may serve as coating for bonding wires and low contact resistance. The combined thickness of the metal layer becomes $20.5 \mu \mathrm{m}$. The combined layer is referred to as metal [103]. The metal layer, as described previously, is used to form the fingers and the curve-up of the actuator. Figure 4-4 illustrates the cross-sectional view of the MetalMUMPs fabrication process. 


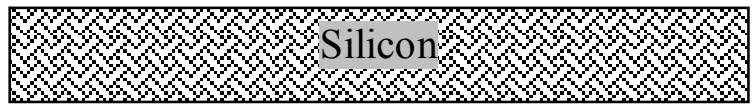

(a) Silicon substrate n-type 100

Nitride 1

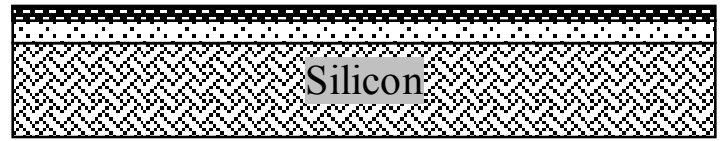

(c) Nitride1 layer

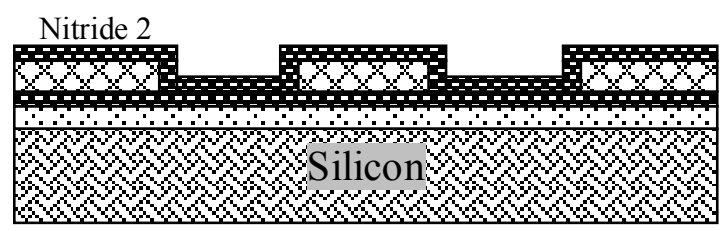

(e) Nitride2 layer

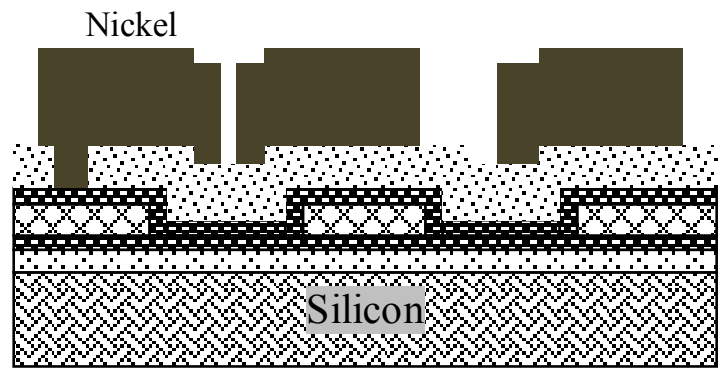

(g) Nickel metal deposited and patterned
Silicon dioxide

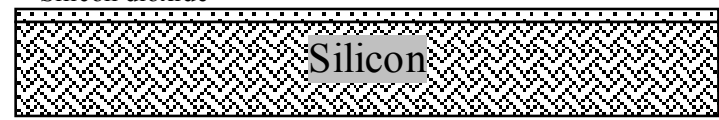

(b) Isolation oxide deposited

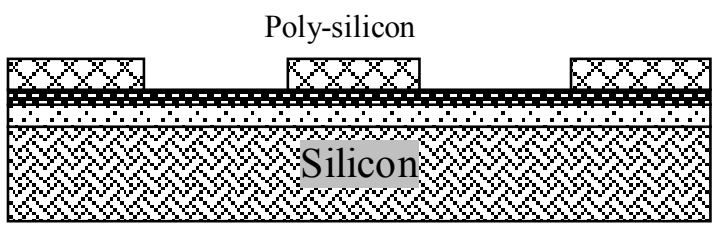

(d) Poly-silicon strips

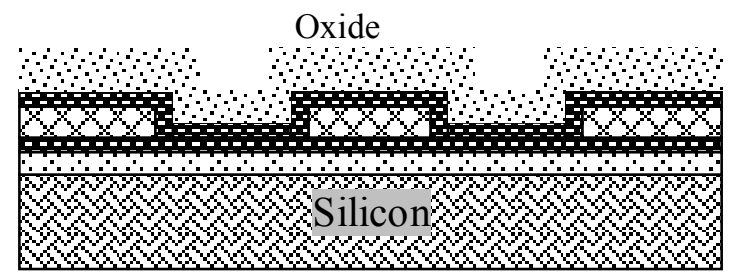

(f) $\mathrm{SiO}_{2}$ sacrificial layer

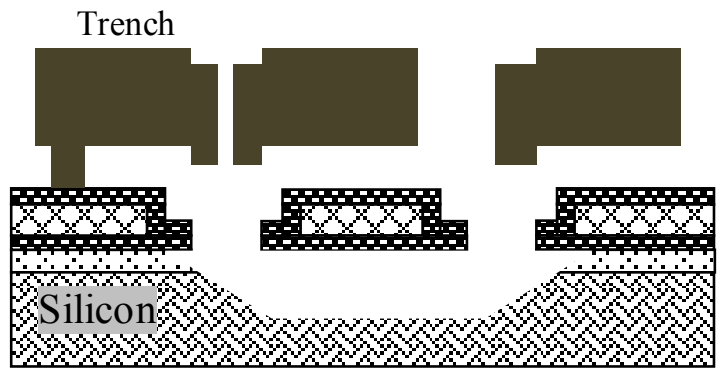

(h) Structures released over trench

Figure 4-4 MetalMUMPs fabrication process flow [103]

\subsubsection{Material properties and Design specifications}

Table 4-1 outlines mechanical and electrical properties of the MetalMUMPs process according to CoventorWare's material database [81]. Table 4-2 illustrates the thickness of specific layer in the process. 
Table 4-1 MetalMUMPs material properties [81, 101]

\begin{tabular}{cccccc}
\hline Material & $\begin{array}{c}\text { Young's modulus } \\
(\mathrm{GPa})\end{array}$ & $\begin{array}{c}\text { Poisson } \\
\text { ratio }\end{array}$ & $\begin{array}{c}\text { Residual Stress } \\
\text { gradient }(\mathrm{MPa} / \mu \mathrm{m})\end{array}$ & $\begin{array}{c}\text { Conductivity } \\
(\mathrm{pS} / \mathrm{um})\end{array}$ & $\begin{array}{c}\text { Dielectric } \\
\text { constant }\end{array}$ \\
\hline Nickel MetalMUMPs & 159 & 0.3 & -4.72 & $1.25 \mathrm{e} 13$ & 0 \\
Silicon nitride 1 \& 2 & 254 & 0.23 & --- & 0 & 8 \\
Poly silicon & 158 & 0.22 & --- & $6.5 \mathrm{e} 10$ & 0 \\
Silicon oxide & 70 & 0.17 & --- & $3.3 \mathrm{e} 13$ & 0 \\
Gold MetalMUMPs & 57 & 0.35 & --- & --- & 0 \\
Chromium films & 140 & 0.3 & --- & & \\
\hline
\end{tabular}

Table 4-2 Layers of MetalMUMPs process

\begin{tabular}{ccccccccc}
\hline Layer & Silicon & $\begin{array}{c}\text { Isolation } \\
\text { oxide }\end{array}$ & Oxide1 & Nitride1 & Poly & Nitride2 & Oxide2 & Nickel \\
\hline Thickness $(\mu \mathrm{m})$ & 675 & 2 & 0.5 & 0.35 & 0.7 & 0.35 & 1.1 & 20 \\
\hline
\end{tabular}

\subsubsection{Simulation of MetalMUMPs clamped-clamped and cantilever beams}

The residual stress gradient is used to analyze the bending of the clamped-clamped and cantilever beams. The dimension of the unstressed clamped-clamped beam is $3000 \mu \mathrm{m} \times 150 \mu \mathrm{m}$. A supporting rectangular spring $300 \mu \mathrm{m}$ long and $10 \mu \mathrm{m}$ wide are connected at the ends of the beams before the anchors. The addition of the spring gives variable overall cross section for the curve up beam. From simulations, beams which have uniform cross section do not achieve upward deflection. A stress gradient value of $-4.72 \mathrm{MPa} / \mu \mathrm{m}[101]$ is used in CoventorWare to simulate the deformation and deflections of the beams. The simulated deformation of a clampedclamped beam is shown in Figure 4-5 and 4-7. For cantilever beams the residual stress gradient deforms the tip downward as shown in Figure 4-6. The free end of the beams attains the highest deflection. In the case of clamped-clamped beams, higher deformation or curvature occurs at the middle of the beam. The maximum displacement depends on the length of the beams or springs by which the beam is fixed or anchored. The dome shaped beams in the thesis are called curved beams or curved-up beams. These names are used interchangeably throughout this thesis work. It has been found that the deflection of the beams is a function of the length of the pre-stressed beam, the type of the spring used to fix the beams, and the dimensions of the spring. 


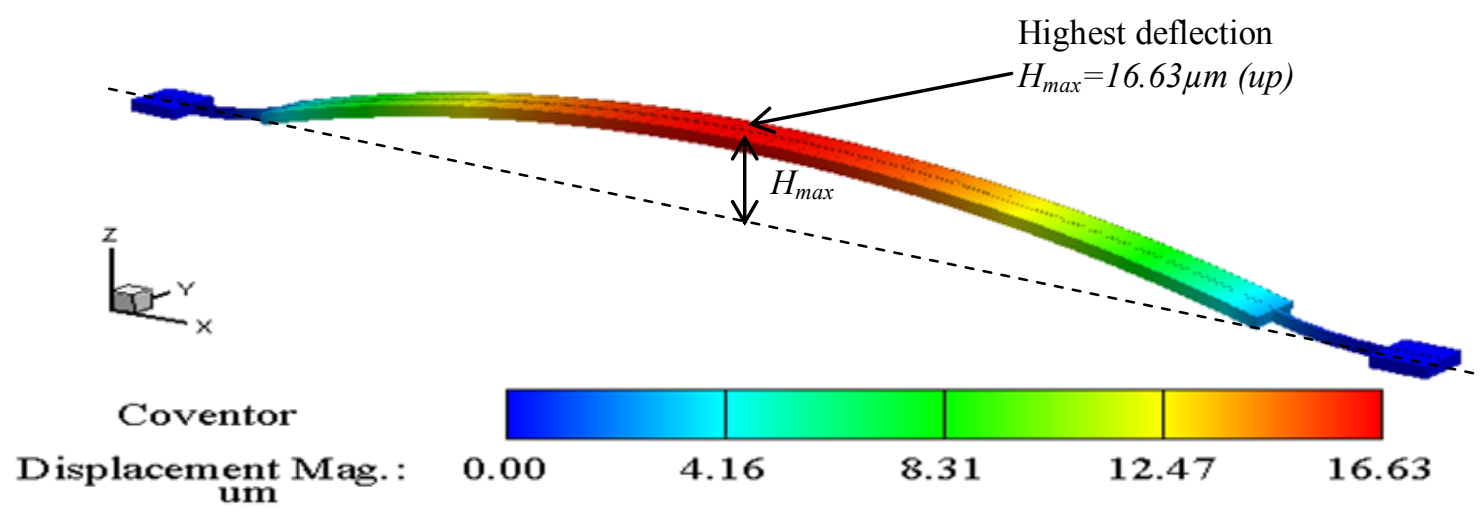

Figure 4-5 Clamped-clamped beam deformations using residual stress gradient

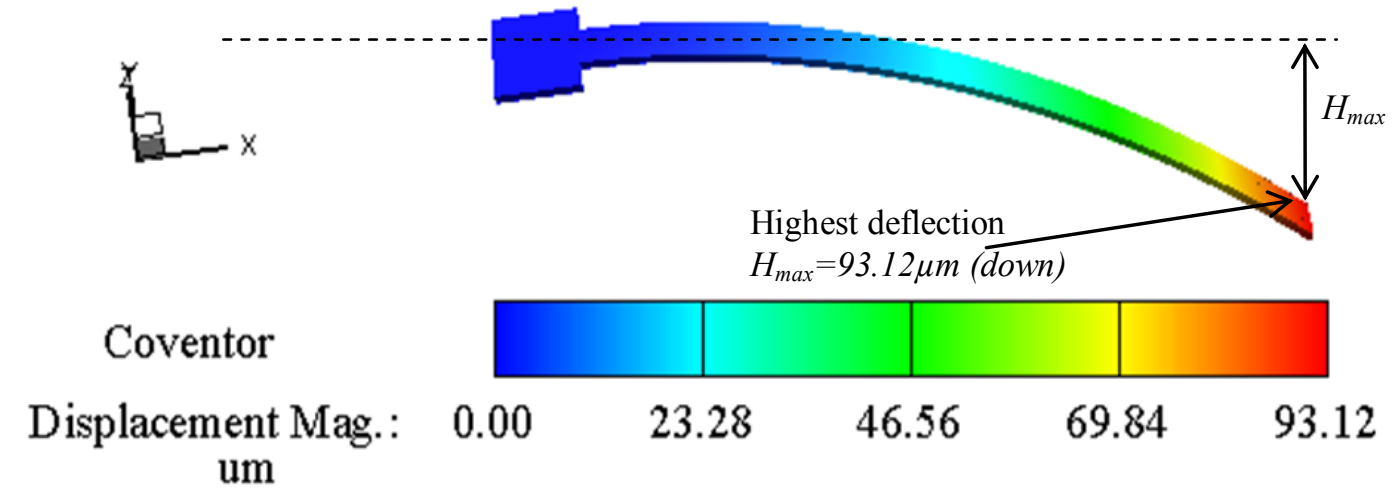

Figure 4-6 Cantilever beam deformations using residual stress gradient

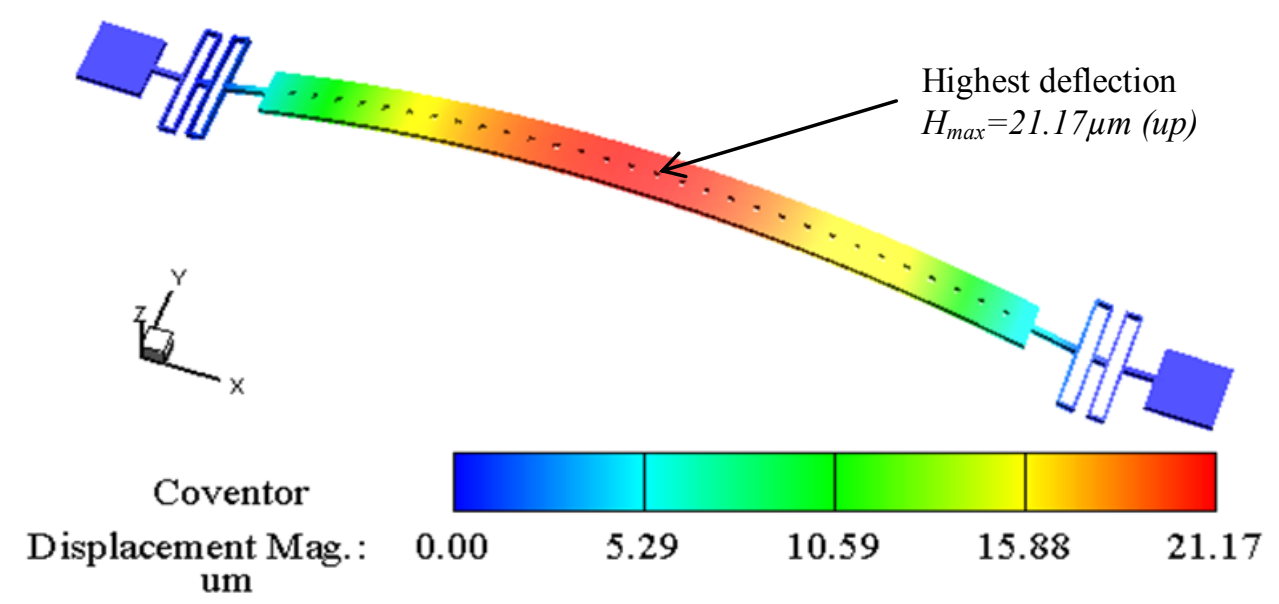

Figure 4-7 Clamped-Clamped anchored using box-type springs 


\subsection{Design principle of vertical comb-drive actuator}

This section discusses the application of the curve up beams in the design of novel vertical comb-drive actuators. As it was explained in section 4.3, clamped-clamped beams bend upward to raise or lift moving fingers of the actuator above the substrate. The 3D representation of the actuator is shown in Figure 4-8.

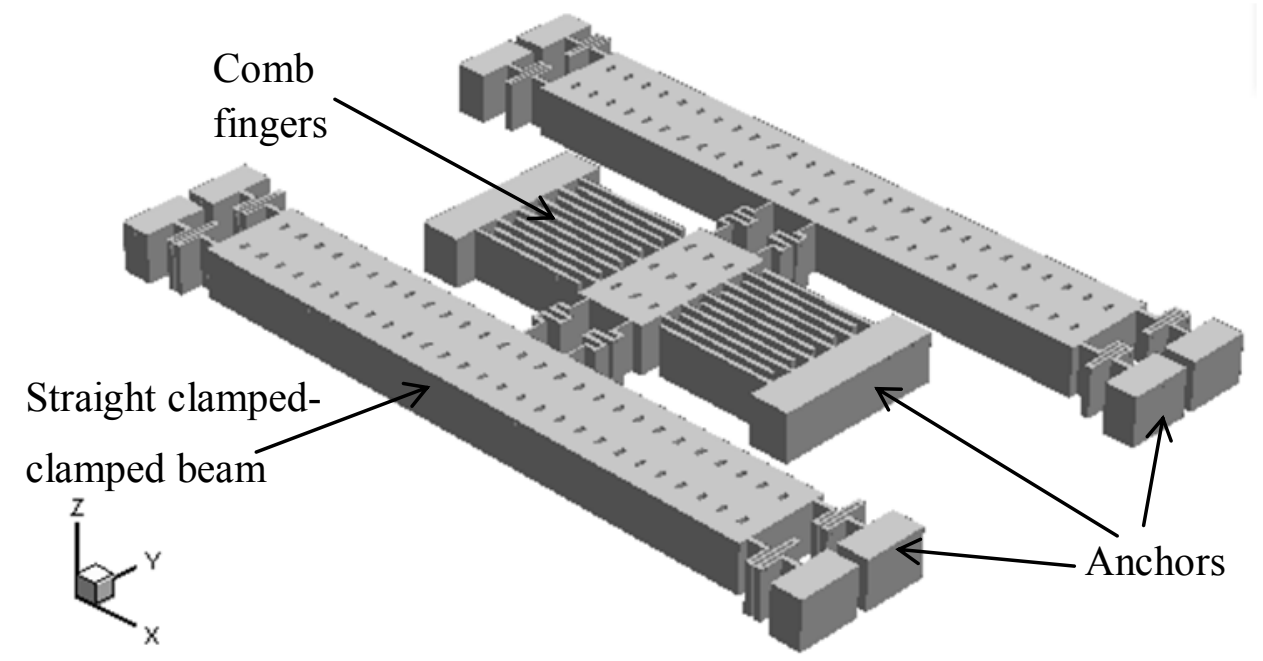

(a) Before residual stress gradient deformation

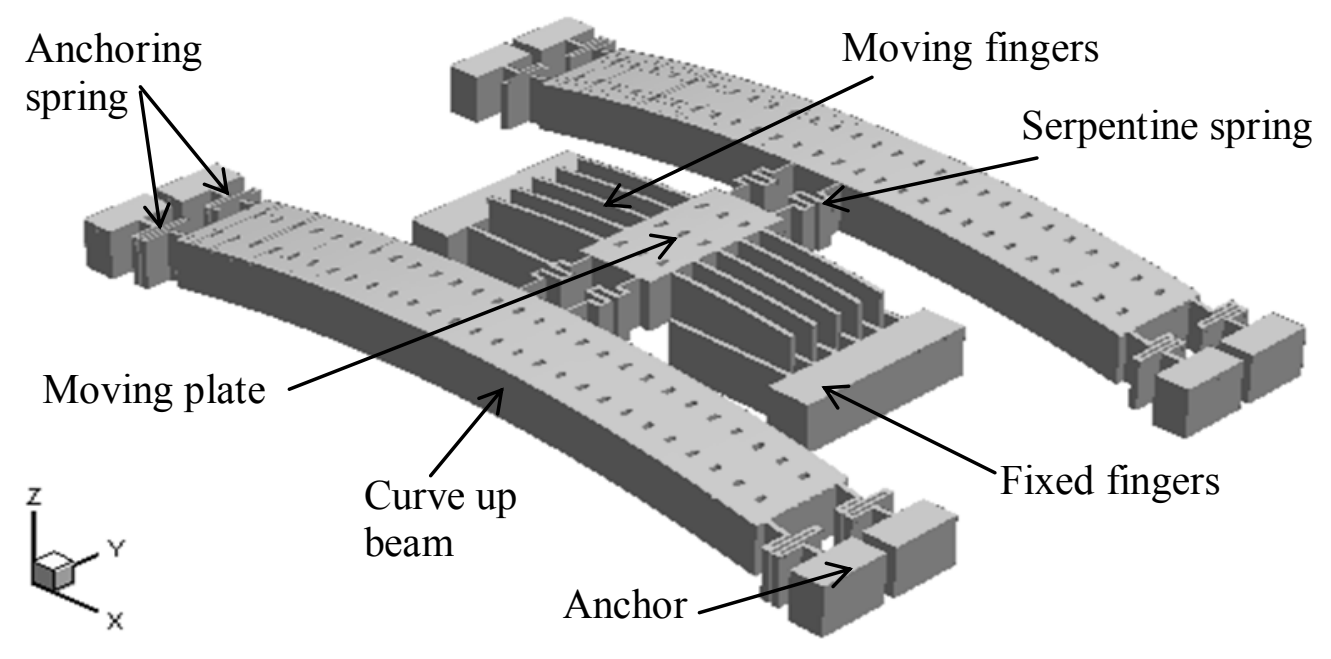

(b) After residual stress gradient deformation

Figure 4-8 Actuator 3D structure 
Two long beams, curve up beams in Figure 4-8, undergo large vertical out of plane deformations and attain a certain height above the substrate. A large plate which is attached to the curve-up beams through serpentine springs around the middle is lifted above the substrate. This plate is known as the moving plate in this thesis. Arrays of fingers connected to the opposite sides of the moving plate. These fingers form the moving fingers of the actuator. The height of the moving fingers above the substrate is determined by deformations that result from the residual stress gradient on the curve up beams. The moving plate and the moving fingers are called moving or suspended structures as a whole. The fixed fingers are attached to the substrate through anchors. Anchors are lithographically patterned non-moveable structures that enable mechanical contact of the curved beams and the fixed fingers to the substrate. Therefore, the moving fingers and the fixed fingers form the comb-drive actuator. The fixed fingers are spaced so that the side walls of the moving fingers and the fixed fingers become parallel to each other and separated by a distance $d$ (Figure 4-9). The distance between the moving fingers and the fixed fingers is chosen with reference to the minimum feature requirement of the MetalMUMPs process design rules.

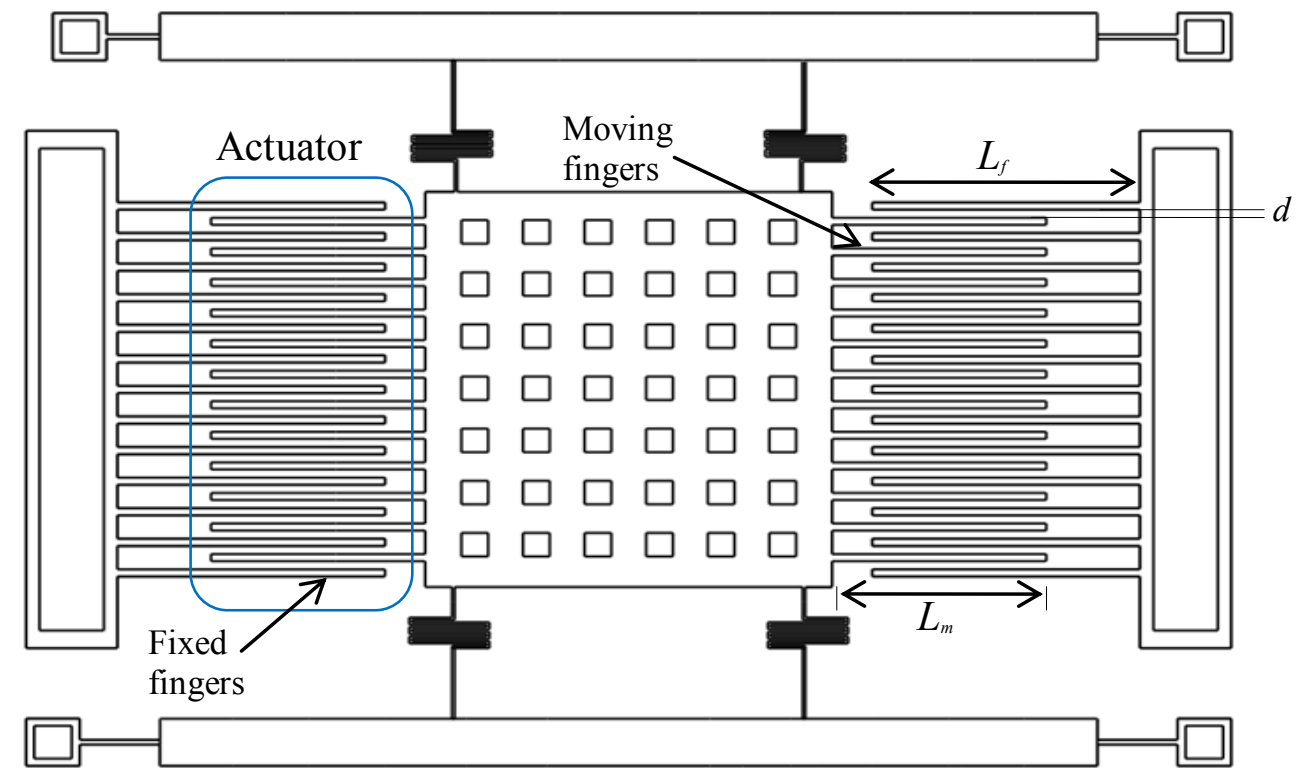

Figure 4-9 Top view of the actuator

When a voltage is applied across the fixed fingers and the moving fingers, the electrostatic force of attraction brings the moving fingers down. Thus, the capacitance between the fixed and moving fingers changes. The capacitance change increases when actuation voltage increases. 
When the voltage is cutoff, the moving fingers return to the original position. For vertical comb drive actuators, the electrostatic force at any actuation voltage, $\mathrm{V}$, is given by:

$$
F_{\text {electrostatic }}=\frac{1}{2} n V^{2} \frac{d C}{d z}
$$

where $n$ is the total number of fingers, $V$ is the voltage across the fingers, $C$ is the capacitance between the moving and the fixed fingers and $z$ is the distance the moving fingers travel when actuated.

The next section exclusively discusses the simulation of the curved beams and the actuators.

\subsection{Actuator layout, 3D models and simulations}

This section discusses the design layout, 3D model generation and simulation of the actuators. Prior to the simulations of the actuator individual curved beams are simulated and the corresponding deformations are analyzed. The 2D layouts of the beams and the actuators were sketched using Autodesk ${ }^{\circledR}$ in DXF format. The DXF formats of the layouts were imported to the CoventorWare $2010^{\circledR}$ designer environment to generate the $3 \mathrm{D}$ model of the beams and actuators.

\subsubsection{Curved beam simulations}

Seven beams with different dimensions are simulated to predetermine the deflection of the curve up beams. The dimensions of the beams are listed in Table 4-3. The actuators are labeled with names B1, B2, B3, B4, B5, B6 and B7. The dimensions listed in the table refer to the nickel and the coating gold layers. The deformations are analyzed based on the length, width and the type of anchoring spring used.

Table 4-3 Dimensions of curve-up beams

\begin{tabular}{cccccccc}
\hline Curved beams & B1 & B2 & B3 & B4 & B5 & B6 & B7 \\
\hline Length $(\mu \mathrm{m})$ & 2000 & 2100 & 2000 & 1820 & 1700 & 2500 & 2500 \\
\hline Anchoring-spring & $210 \times 10$ & $210 \times 10$ & $240 \times 10$ & $330 \times 10$ & $360 \times 10$ & $210 \times 16$ & $210 \times 10$ \\
$($ Length $\times$ Width $)$ & Rectangular & Rectangular & Serpentine & Rectangular & Rectangular & Rectangular & Serpentine \\
\hline
\end{tabular}


Two types of springs are used to anchor the curve-up beams and the deformations are compared. The anchoring springs used are rectangular and serpentine types. The shapes of the springs are illustrated in Figure 4-10.

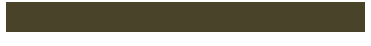

(a)

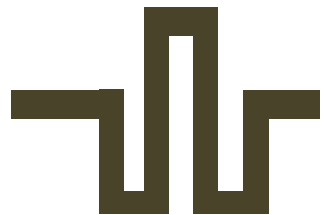

(b)

Figure 4-10 Anchoring springs (a) rectangular (b) serpentine

After the 3D models of the beams were generated, the residual stress gradient on the nickel of MetalMUMPs was inserted into the mechanical solver of Coventor. The anchors of the clampedclamped beams of the capacitor beams are fixed.

S. He et al [101] experimentally determined the residual stress gradient on cantilever beams made of the MetalMUMPs metal layer. The values of the stress gradients determined however vary for each cantilever or fixed-fixed beams used in the experiment. This variation may be due to the non-uniform thickness of the fabricated beams of the metal. The result in the paper showed that the residual stress gradient varies along the thickness of the nickel film. The average residual stress gradient of $-4.72 \mathrm{MPa} / \mu \mathrm{m}$ along z-axis is used to analyze the deformations of the beams. Figure 4-11 shows deformation a rectangular spring anchored clamped-clamped beam.

In the simulations a beams anchored with rectangular anchoring springs areas were deflected more than the beams which are not anchored with thin rectangular beam. Figure 4-11 shows the simulated curve-up beam which consists of rectangular anchoring spring and the main beam. The difference in the cross sectional area enabled the beam to achieve large out of plane deflection. Beams with uniform cross section area are stiff and do not achieve considerable deflection. Therefore, beams anchored with thin springs provide low stiffness to yield significant out of plane deflection. Clamped-clamped beams with uniform cross sectional areas have negligible deflection. Thus in order to obtain large deflection anchoring springs is added at the ends of the curve-up beams. 


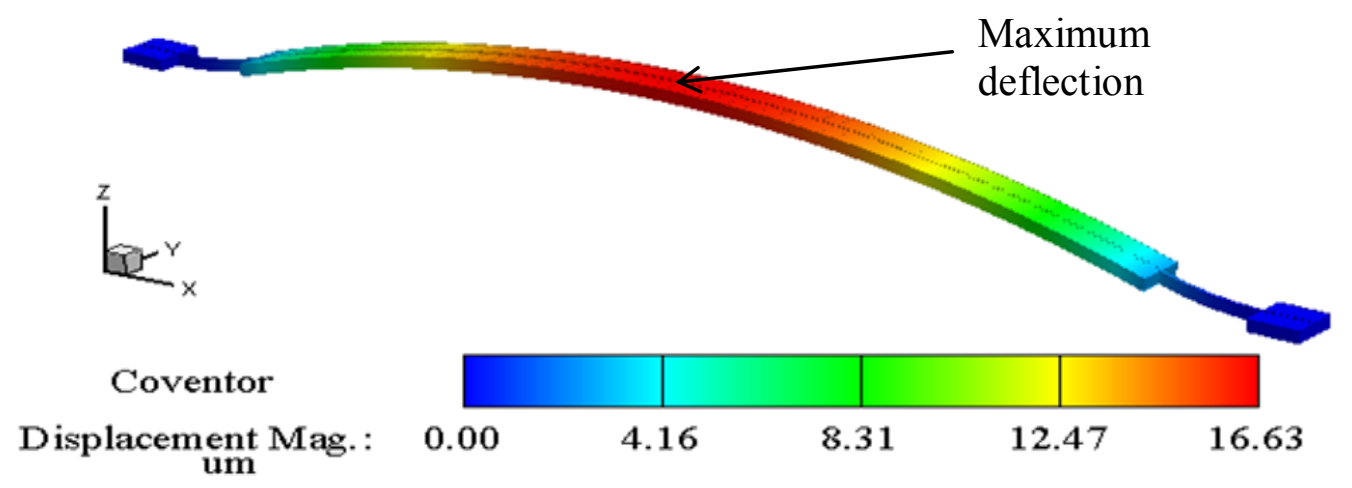

Figure 4-11 Simulated beam of clamped-clamped beam

The graph shown in Figure 4-12 shows the relationship between the length and the maximum deflection of the beams. The length of the beam includes the length of the anchoring springs. The deflection of the beams refers to the maximum displacement that occurs at the geometric center of the curve-up beams.

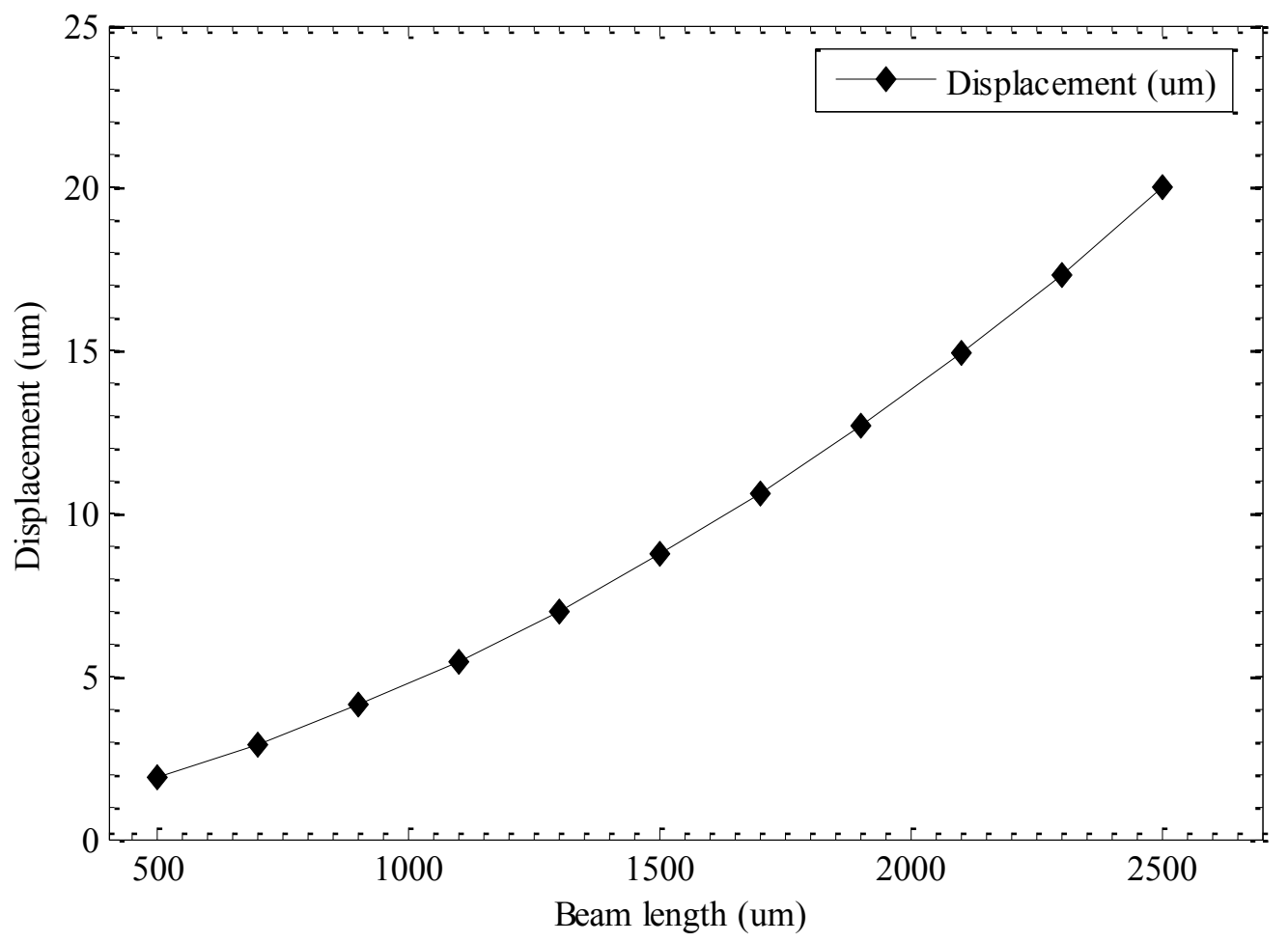

Figure 4-12 Maximum deflection of curve-up beams vs. length 
The analysis showed that longer beams produced large deflection at the middle of the beam. The width of the beam causes negligible change compared with the length. However, significant increment of the width of the beams causes observable increase to the vertical deflection of the beam. For instance, if the width of the beam is doubled while the beam and anchoring spring parameters unchanged, the deflection can be increased by $40 \%$. Table $4-4$ shows the simulated deflection of the curve-up beams.

The type of the anchoring spring significantly affects the maximum deflection of the beams. Rectangular anchoring springs result in smaller deflection compared with serpentine spring. It was found that thinner anchoring springs either serpentine or rectangular type have low springs constant and give higher deflections.

Table 4-4 Simulated and measured heights of moving finger/plate

\begin{tabular}{cccccccc}
\hline Curve-up beams & B1 & B2 & B3 & B4 & B5 & B6 & B7 \\
\hline Simulated deflection $(\mu \mathrm{m})$ & 6.70 & 7.12 & 11.40 & 7.28 & 6.50 & 6.85 & 15.77 \\
\hline
\end{tabular}

The profiles (arch shapes) of the curve up beams after simulation are plotted in Figure 4-13. The curves shown are plotted by extracting data points from the deformed beams. The curve-up beams are named using the names of the actuators. Beam B1 represents the deformation of the beam used to design Actuator 1 and so on. The curve beams B3 and B7 have serpentine anchoring springs while the beams B1, B2, B4, B5 and B6 are anchored with rectangular springs. Data points are extracted from the curve-up beam deformations of CoventorWare simulations. The data points are the coordinates on the deformed beams between the anchors of the beams including the anchoring springs. The beams deformations plotted in Figure 4-13 are curve fitted to find a function that relates the length of the beam to the achievable deflection along the length of the beams. The equations can be used to determine the arc length of the curve up beams. The curve fitting of Matlab ${ }^{\circledR} \mathrm{R} 2012 \mathrm{~b}$ [72] is used to determine the coefficients of the equation. The polynomial function of degree- 6 fits well to the extracted data over the length of the beam including the length of the anchoring springs with minimal norm of residuals of 7.7608.

$$
f(z)=p_{1} z^{6}+p_{2} z^{5}+p_{3} z^{4}+p_{4} z^{3}+p_{5} z^{2}+p_{6} z+p_{7}
$$

For curve-up beam B7, Figure 4-14, the coefficients of the polynomial are:

$$
p_{1}=0.86954, p_{2}=-0.00040367, p_{3}=-1.37, p_{4}=9.3085 e-5 \text {, }
$$


$p_{5}=-7.6902, p_{6}=0.0027963, p_{7}=15.906$

For curve-up beam B1 the fitted curve is shown in Figure 4-15 with the coefficients of 6-degree polynomial: $\quad p_{1}=0.2785, p_{2}=0.015256, p_{3}=-0.79998, p_{4}=-0.033069, p_{5}=-2.2842$, $p_{6}=-0.02796 p_{7}=6.4492$ and norm of residuals $=1.5338$. Similarly, the fitting polynomials for the beams of Actuator 2 to Actuator 6 are shown in the Appendix A.

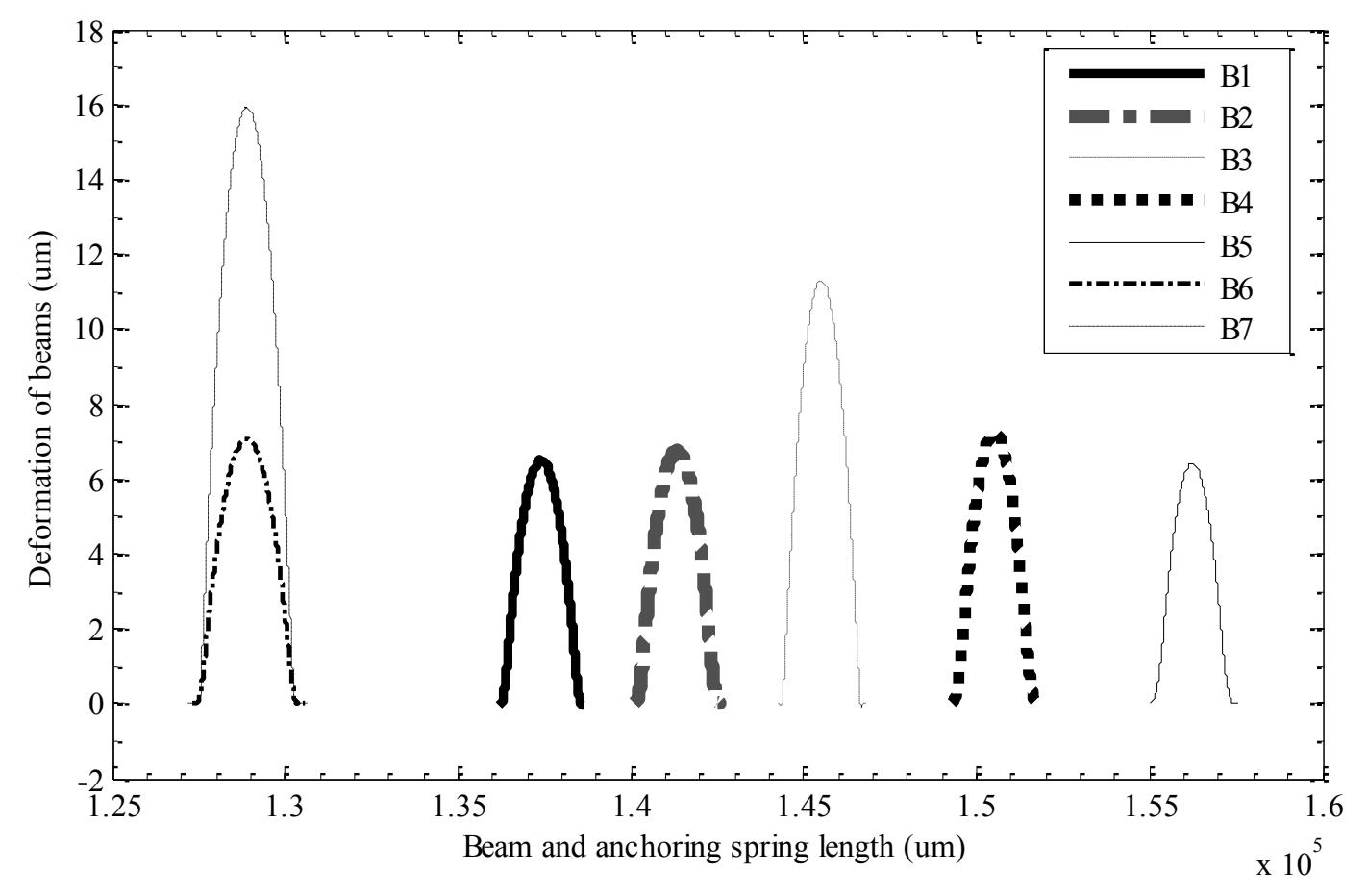

Figure 4-13 Plots of shapes of curved-up beams B1-B7 


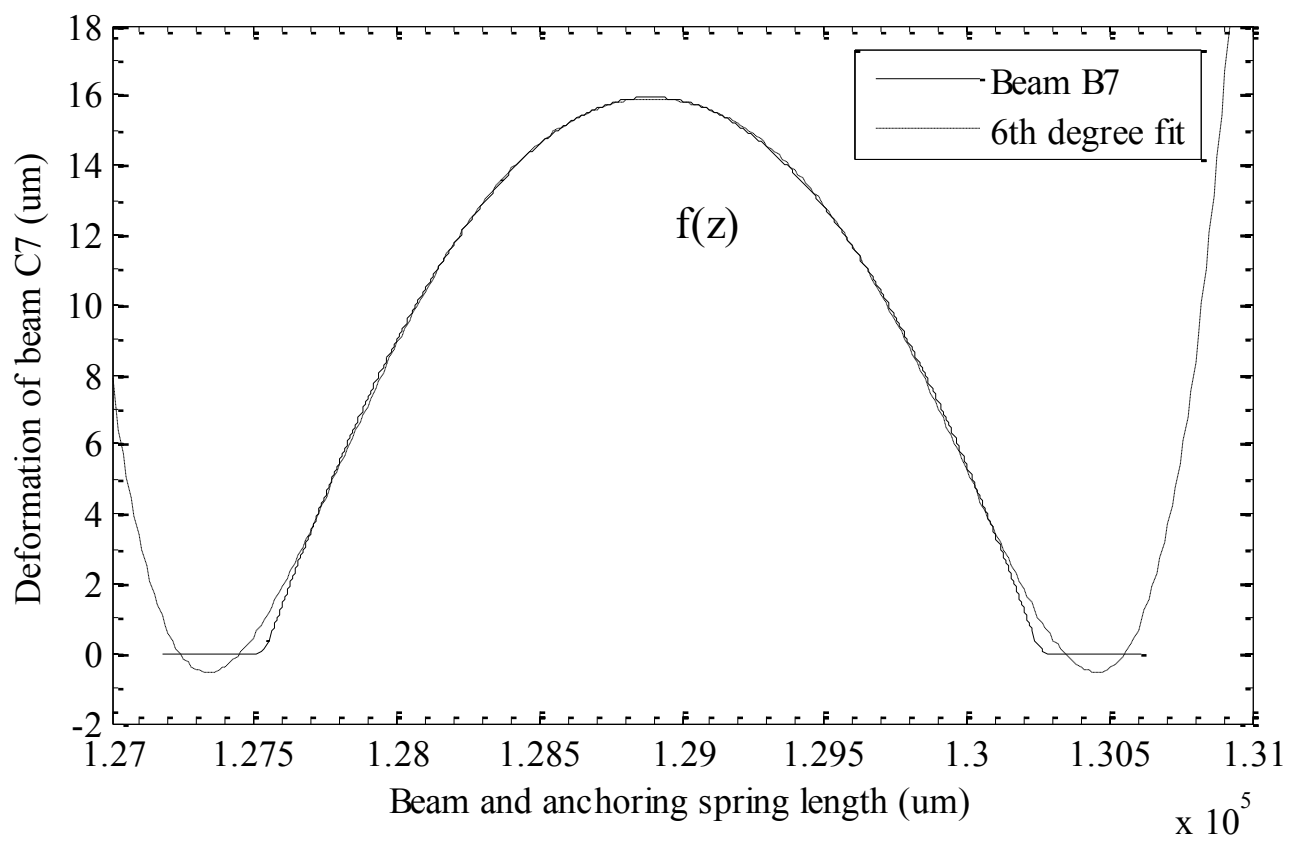

Figure 4-14 Polynomials fitting of the deformation of beam B7

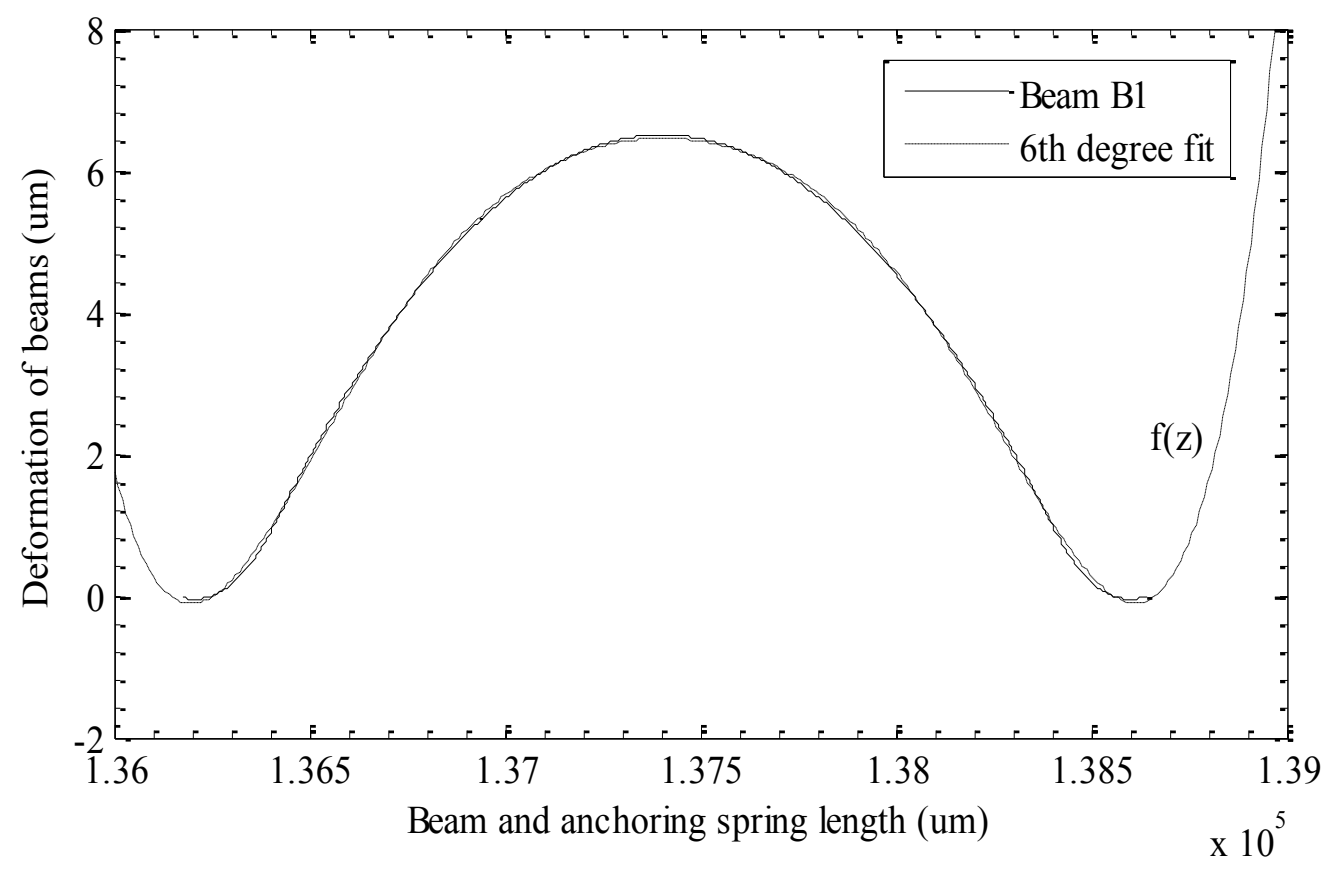

Figure 4-15 Polynomial fitting of the deformation of beam B1 The length of the curved beams can be evaluated using the arc length formula [110]; 


$$
L_{\text {curved beam }}=\int_{0}^{L} \sqrt{1+\left(\frac{d f}{d z}\right)^{2}} d z
$$

$L$ is the length of the length of the non-deformed beams including the length of the anchoring springs before deformation occurs. $d f$ is the first order derivative of the fitted polynomial with respect to the length $z$.

\subsubsection{Actuator design}

The general configurations of the comb drive actuators are illustrated in this section. In Figure 4-16, the 2D layout of actuator with rectangular anchoring spring is shown with its key parts labeled. Figure 4-17 shows the actuator's 3D view which was generated using CoventorWare's MetalMUMPs foundry. The actuator design now consists of two curve-up beams flanking the moving plate, the moving fingers, the fixed fingers and the plate supporting springs. Pads and strips are also included for DC biasing during prototype testing. The actuator designs are broadly categorized into two groups based on the type of spring used to anchor the curve up beams: rectangular anchoring springs and serpentine anchoring springs. Figure 4-17 shows the 3D view of the actuator with cantilever type anchoring springs and the trench in the substrate using CoventorWare preprocessor editor. 


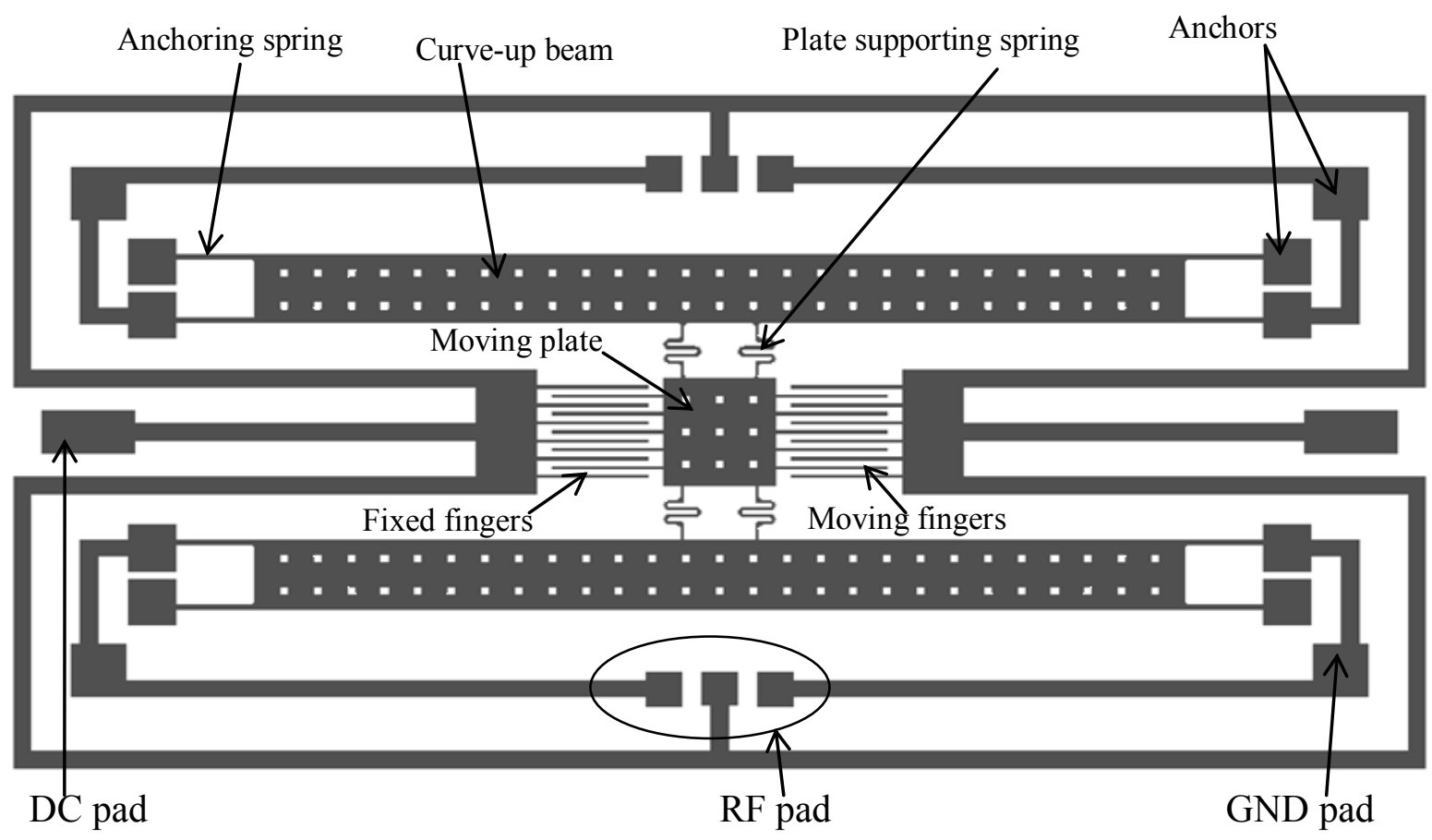

Figure 4-16 Actuator 2D layouts with rectangular anchoring spring
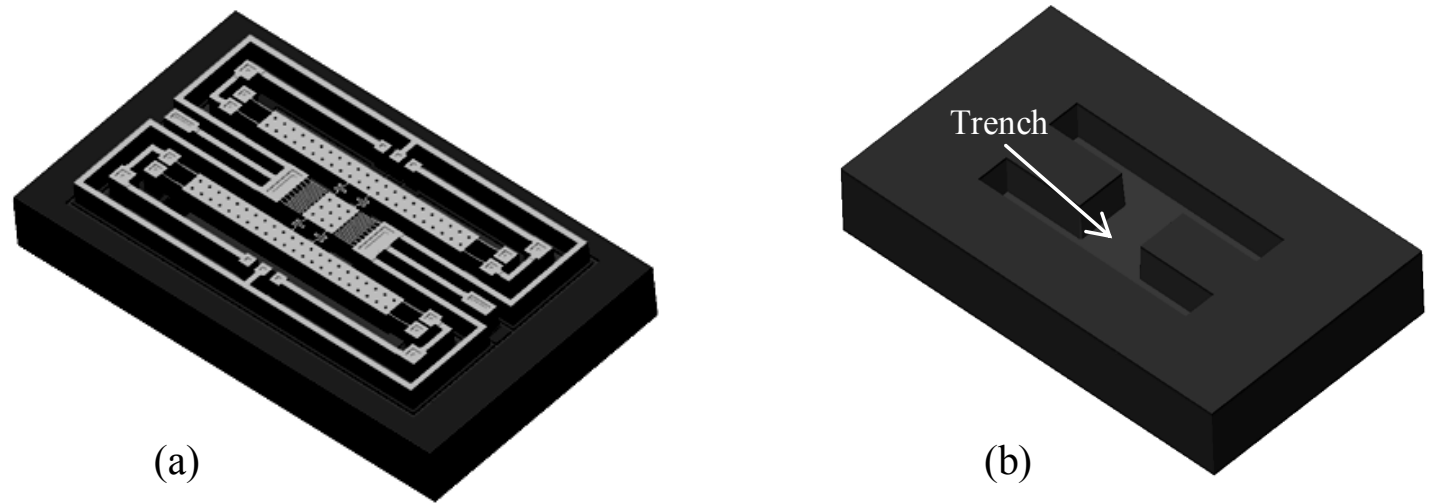

Figure 4-17 Actuator 3D models with (a) complete structure (b) trench in substrate

\subsubsection{Actuators dimensions}

Seven actuators A1 to A7 with different geometry are designed and simulated. Table 4-5 shows all parameters of each actuator. 
Table 4-5 Actuator dimensions (in micrometers)

\begin{tabular}{ccccccccc}
\hline \hline Actuator & & $\begin{array}{c}\text { Moving } \\
\text { finger }\end{array}$ & $\begin{array}{c}\text { Fixed } \\
\text { fingers }\end{array}$ & $\begin{array}{c}\text { PM } \\
\text { spring }\end{array}$ & $\begin{array}{c}\text { Curve-up } \\
\text { beam }\end{array}$ & $\begin{array}{c}\text { Moving } \\
\text { plate size }\end{array}$ & $\begin{array}{c}\text { Gap b/n } \\
\text { fingers }\end{array}$ & $\begin{array}{c}\text { Anchoring } \\
\text { springs }\end{array}$ \\
\hline \multirow{2}{*}{ A1 } & L & 170 & 200 & 150 & 2000 & $300 \times 300$ & 10 & 210 \\
& W & 10 & 10 & $100 / 10$ & 200 & & & 10 \\
& L & 400 & 400 & 150 & 2100 & $300 \times 300$ & 8 & 210 \\
A2 & W & 10 & 10 & $100 / 10$ & 200 & & & 10 \\
& L & 500 & 500 & 150 & 2000 & $300 \times 300$ & 12 & $240 \mathrm{~S}$ \\
A3 & W & 10 & 10 & $100 / 10$ & 200 & & & 10 \\
& L & 300 & 300 & 150 & 1820 & $300 \times 300$ & 10 & 330 \\
A4 & W & 10 & 10 & $100 / 10$ & 200 & & & 10 \\
& L & 370 & 400 & 170 & 1700 & $300 \times 300$ & 10 & 360 \\
A5 & W & 10 & 10 & $100 / 10$ & 160 & & & 10 \\
\hline \multirow{2}{*}{ A6 } & L & 300 & 300 & 153 & 2500 & $300 \times 300$ & 15 & 210 \\
& W & 10 & 10 & 10 & 194 & & & 16 \\
A7 & L & 500 & 500 & 153 & 2500 & $300 \times 300$ & 15 & $210 \mathrm{~S}$ \\
& W & 10 & 10 & 10 & 194 & & & 10 \\
\hline \hline
\end{tabular}

Notation: $\mathrm{L}=$ length and $\mathrm{W}=$ width, $\mathrm{PM}$ spring= moving plate spring $\mathrm{S}=$ serpentine spring

The moving plate is common for all actuator designs with an area of $300 \times 300 \mu \mathrm{m}^{2}$. The curve up beams for actuators A1, A2, and A3 have $2000 \mu \mathrm{m}$ long and $200 \mu \mathrm{m}$ wide beam with rectangular anchoring springs. The main deference among the actuators $\mathrm{A} 1, \mathrm{~A} 2$ and $\mathrm{A} 3$ are the gap between the moving and the fixed fingers, and length of the fingers. The curve up beams and the moving plates are provided with holes of area $40 \times 40 \mu \mathrm{m}^{2}$ for etching the sacrificial layer under the metal layer. The actuators A4 and A5 nearly have identical beam length with different widths while the anchoring springs are rectangular and longer compared with A1 and A2. Actuators A6 and A7 have longer curve up beams of length $\geq 2500 \mu \mathrm{m}$ and width $194 \mu \mathrm{m}$ with rectangular and serpentine anchoring springs respectively. The gap between the fixed and the moving fingers of A6 and A7 is $15 \mu \mathrm{m}$. The curve up beams A3 and A7 are also provided with serpentine anchoring springs to achieve higher deflection. 


\subsubsection{Simulations of the actuators}

This section analyzes the maximum deflection reached by the moving plate and the moving fingers of the actuators based on the geometry of the curve up beams and the anchoring springs. The substrate and essential structures such as anchors and nitrides are purposely omitted from the simulation to shorten the time taken for computation. The simulated maximum deflections, $H_{\max }$, are listed in Table 4-6. The maximum deflections are the height differences between the moving and the fixed fingers. The surface maps of the actuators A1, A2 and A7 are illustrated in Figure 4-18 (a) to (c). The moving fingers and the moving plates are raised above the substrate and the fixed fingers. The maximum deflections, $H_{\max }$, of the actuators are marked on the surface maps. It refers to the maximum deflection (or simulated height) the moving fingers and the moving plate reach due to the stress gradient deformation after fabrication.

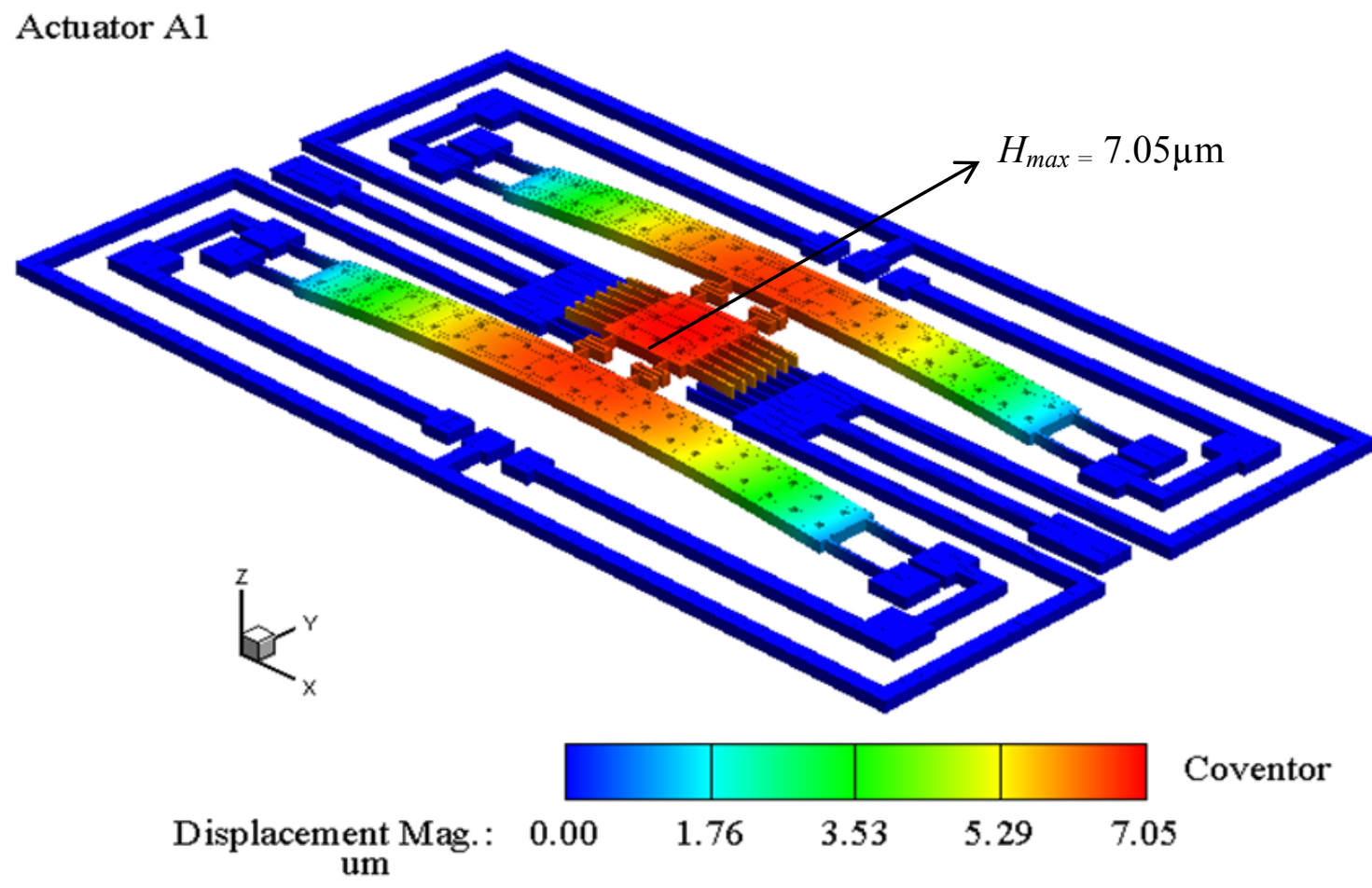

(a) 

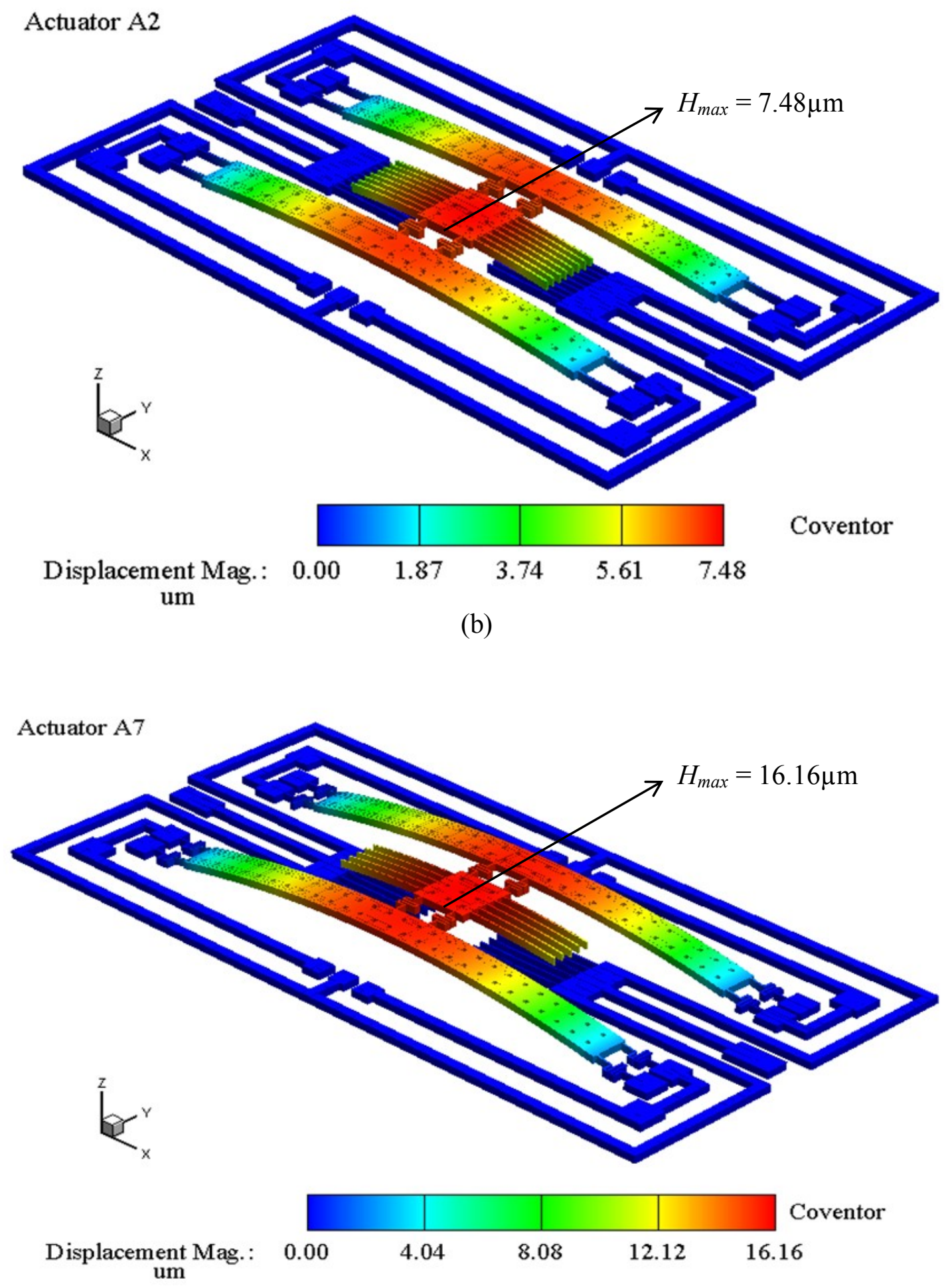

(c)

Figure 4-18 3D maps of analysis results of actuator after stress gradient deformation 
Table 4-6 Simulated heights $\left(H_{\max }\right)$ of moving finger/plate

\begin{tabular}{cccccccc}
\hline Actuator & A1 & A2 & A3 & A4 & A5 & A6 & A7 \\
Simulated deflection $(\mu \mathrm{m})$ & 7.05 & 7.48 & 11.75 & 7.63 & 6.85 & 7.20 & 16.16 \\
\hline
\end{tabular}

\subsection{Prototypes and measurements}

Prototypes of the actuator were fabricated to experimentally verify the design and working principle. In the measurements of the prototypes vertical misalignment between the actuators fingers and the height by which the curve up beams and the moving structures deflected above the substrate are discussed. Inspections of the actuators were conducted using optical profilemeter to measure the surface maps of the actuator and generate the profile plots so that the heights of the moving fingers, the moving plates, the fixed fingers and the substrates. The results of these measurements provide information whether the curve up beams are deflected above the substrate to lift up the moving structures above the substrate. These measurements are called novoltage or voltage-free measurements in this thesis. In other words these measurements are taken without actuation voltage.

The measurements without actuation provide data of the heights of the moving structures above the substrate and the deformations of the curve up beams over various areas and the deflections after fabrication. The data collected is compared with the simulated results $\left(H_{\max }\right)$ obtained using the residual stress gradient parameter. The height difference between the fixed fingers and the moving fingers are also measured. The no-voltage measurement validates the design principles.

On the other hand the measurements with actuation voltage are performed to measure the downward displacement of the moving fingers when a voltage is applied across the moving fingers and the fixed fingers of the actuators. This measurement verifies the working principle of the actuator. The experimental setup described in Section 3.6.1 is used for measuring and testing the actuators.

\subsubsection{Measurement without DC voltage}

The actuators were fabricated on two loose dies with $5 \times 10 \mathrm{~mm}^{2}$ (Chip 1) and $5 \times 5 \mathrm{~mm}^{2}$ (Chip 2) area. Chip 1 has five actuators (A1-A5) and Chip 2 has two actuators (A6 and A7). The actuators are labeled from A1 to A7 and the loose die images are illustrated in Figure 4-19 (a) and (b). 
Figure 4-20 shows the SEM image of the Actuator A7 showing the names of parts of the moving and the fixed structures.
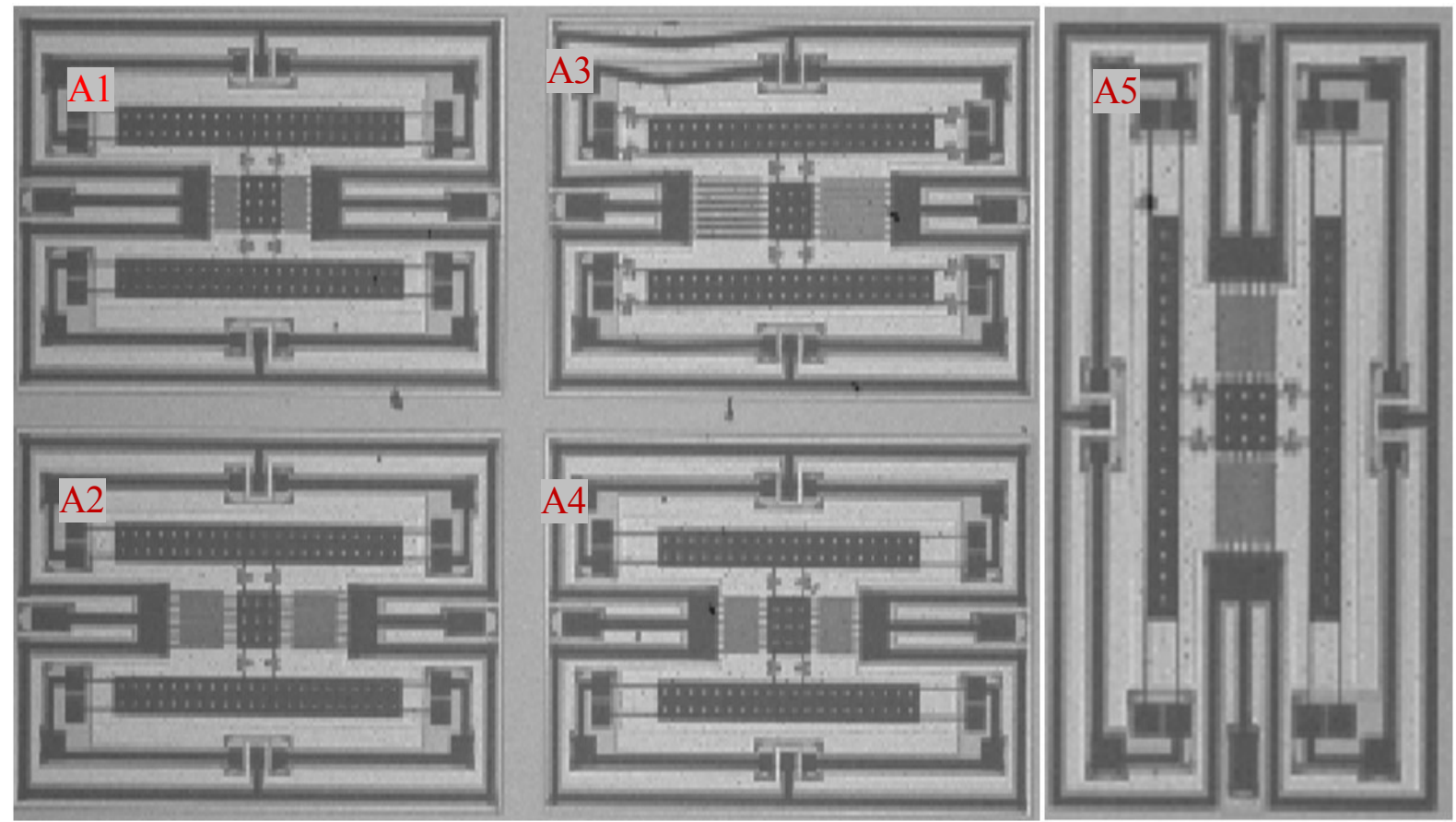

(a)

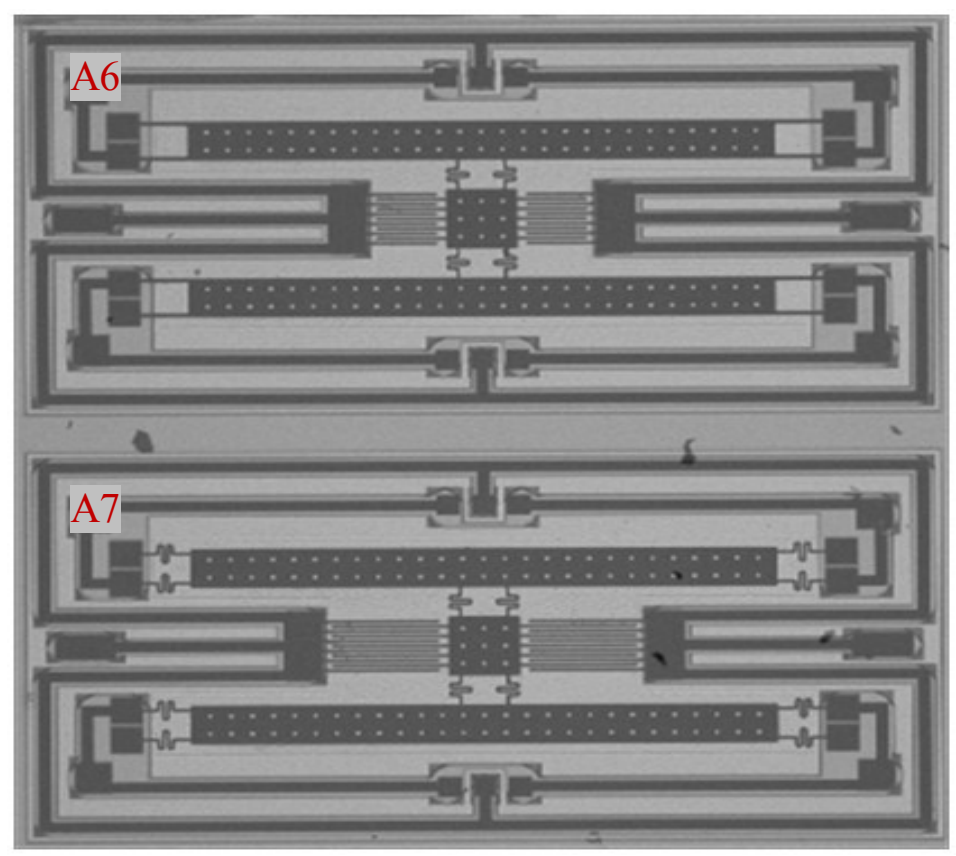

(b)

Figure 4-19 (a) Chip 1(A1, A2, A3, A4, A5) and (b) Chip 2 (A6, A7) 


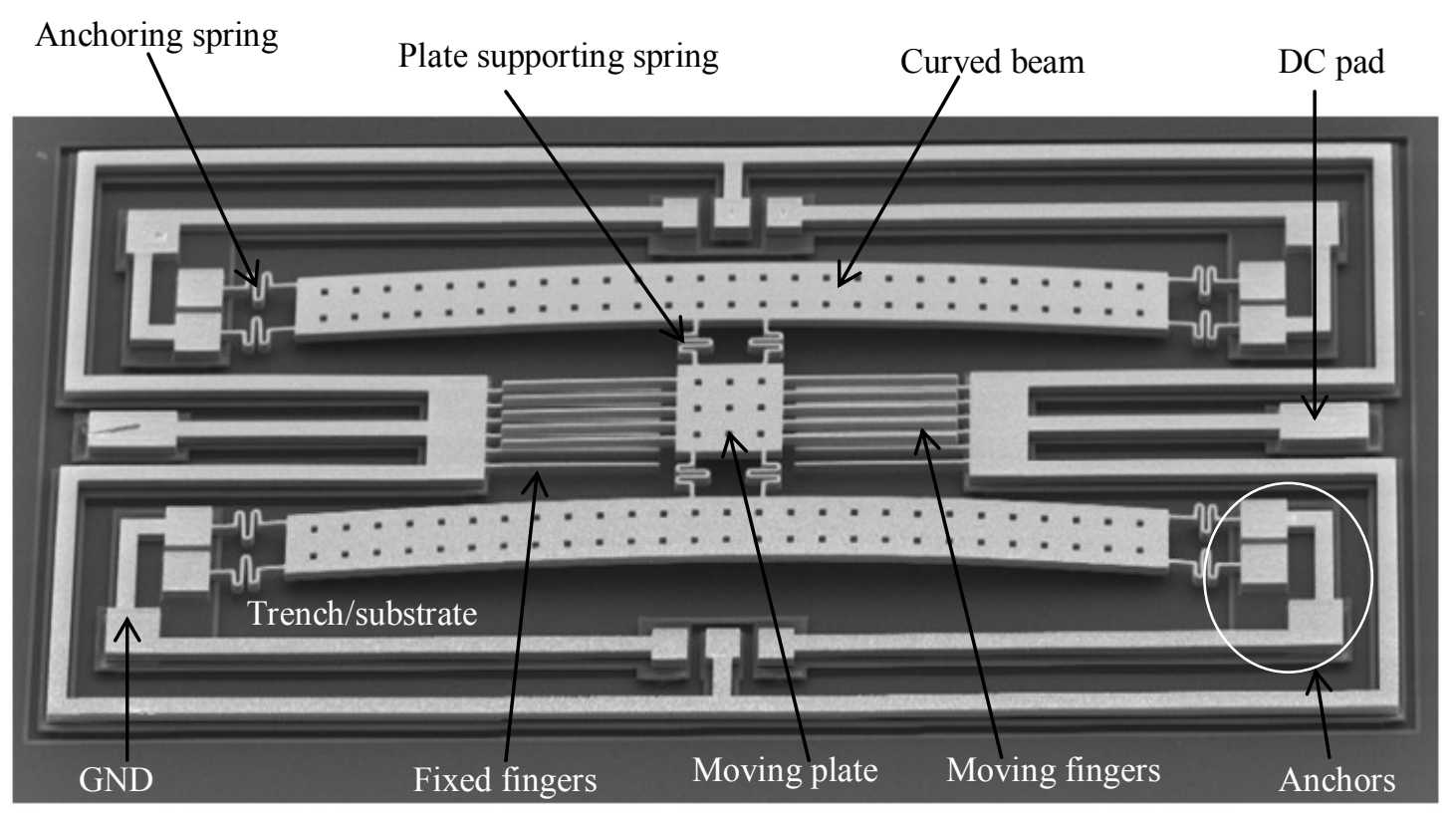

(a)

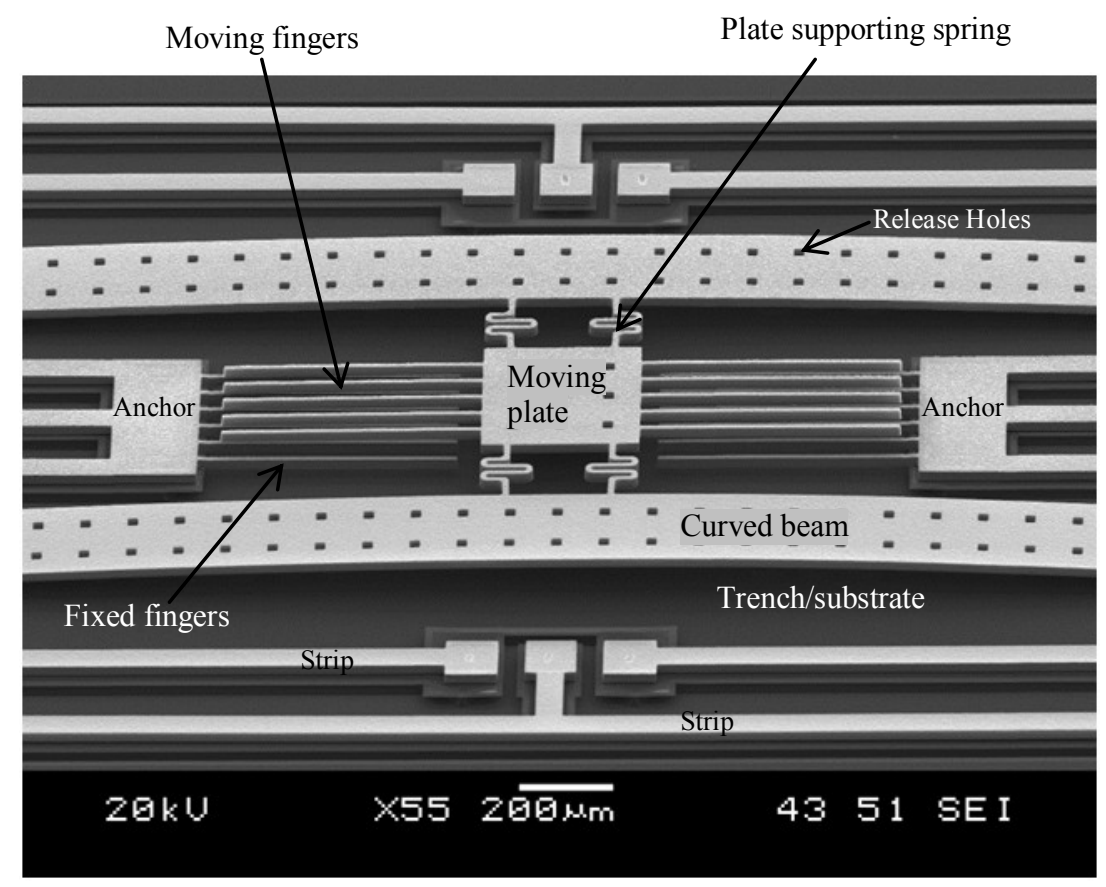

(b)

Figure 4-20 SEM micrograph of Actuator, A7, (a) complete view and (b) magnified view 


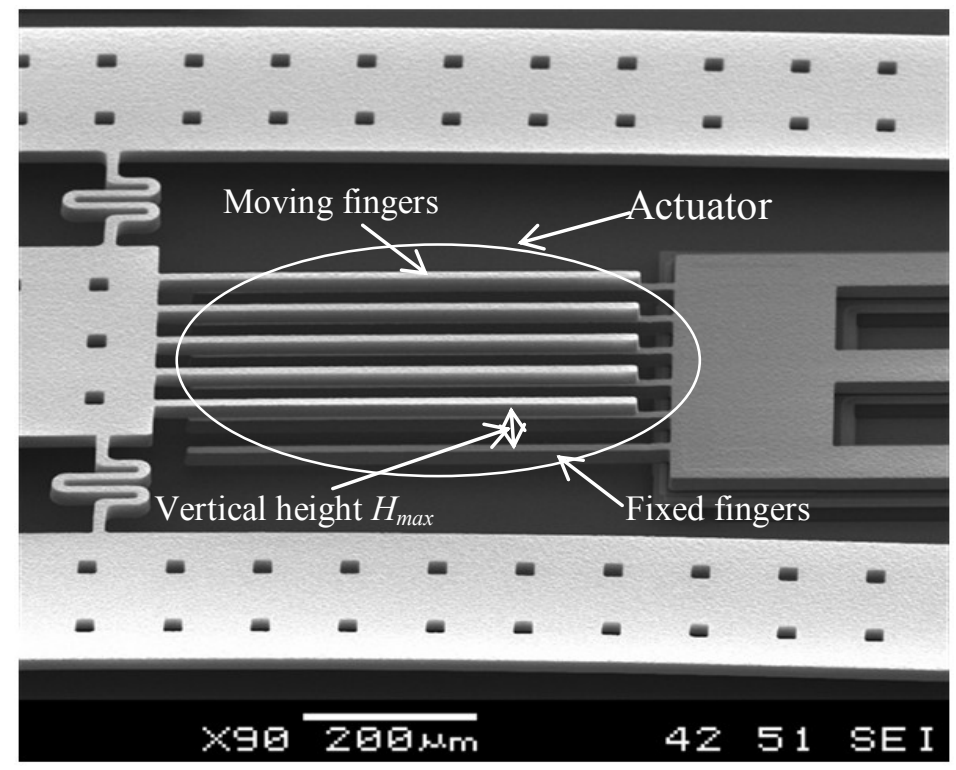

Figure 4-21 Vertical height $H_{\max }$ between moving fingers and fixed fingers

The maximum height, $H_{\max }$, obtained between the fixed and the moving fingers is shown in Figure 4-21.

The measurement readings from the optical profiler are taken using the surface of the trench as a reference plane. However, in this experiment, partial images of the actuators were analyzed on the Metro-Pro image analyzer control windows. This is because the lens field of view (FOV) does not allow full image capture on the Metro-Pro analyzer.

After the analysis of all the surface maps and profiles of the actuators, the measured height $\left(H_{\max }\right)$ between the moving and the fixed fingers are listed in Table 4-7. The measured results are compared with the simulated results and the deviations of the measured results from the simulated results are calculated as percentage errors.

Table 4-7 Error percentage between measured and simulated deflections

\begin{tabular}{llllllll}
\hline Actuator & A1 & A2 & A3 & A4 & A5 & A6 & A7 \\
\hline Simulated $\left(H_{\max }\right)$ & 7.05 & 7.47 & 11.75 & 7.63 & 6.85 & 7.20 & 16.16 \\
Measured $\left(H_{\max }\right)$ & 3.9 & 3.4 & 10 & 4.5 & 4.1 & 3.5 & 15.2 \\
Error (\%) & 44.7 & 54.5 & 14.9 & 41 & 35 & 51.4 & 5.94 \\
\hline
\end{tabular}


Actuators A7 has reached at the highest deflection compared with the other six actuators. Actuator A3 has better relatively deflection among the remaining actuators. The actuators A1, A2, A4, A5 and A6 have shown over 35\% error. Figure 4-22 shows actuator A6 whose comb fingers are not lifted up above the substrate.

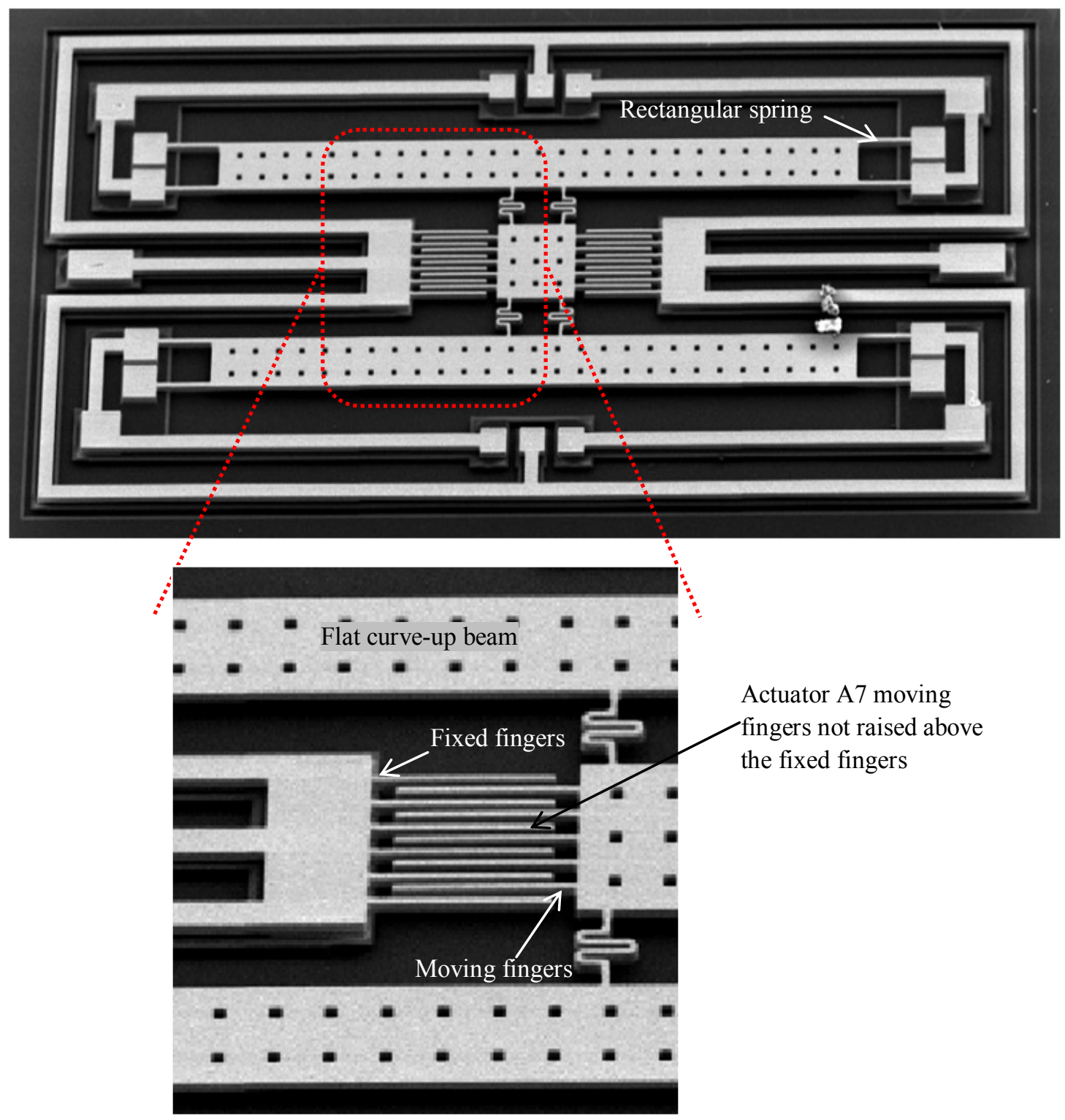

Figure 4-22 Actuator A6 moving fingers not raised above the substrate

In Table 4-7 the simulated results and the measurement results show large error. The factors that caused such errors depend on the fabrication process. The fabrication process parameters at the time of fabrication could affect and change the properties such as the Young's modulus of the 
nickel. The fabricated actuator structures could achieve increased stiffness as a result. Because of the non-uniform deposition of the MetalMUMPs process, the thickness of the nickel films are variable. The thickness of the deposited nickel varies from one loose die to another [101]. The density of the nickel film also varies among the actuator designs on the same loose die and depends on the area covered by the metal layer [103]. The non-uniform deposition and other process parameters (e.g. temperature) affect the thickness and the elasticity of the nickel. The thicknesses of the fabricated actuators differ from the nominal thickness values used in the simulation. The metal thickness ranges from $17 \mu \mathrm{m}-23 \mu \mathrm{m}$ [103]. However, the nominal thickness used for the simulated $H_{\max }$ values is $20.5 \mu \mathrm{m}$ including the coating gold. The thicker films offer larger deflection compared with the thinner films under the same value of stress gradient as studied in simulations. As the thickness varies, the residual stress gradients are expected to change. For thicker nickel films, the residual stress gradients decreases [101] and the achievable maximum deflection is low compared to the nominal thickness. As a result of the above factors, the measured results show larger deviation from the simulated deflections. Sagging effect could also lower the achievable maximum deflection of the curve up beams. There is a possibility that the curve up beams could collapse on the substrate or the trench after fabrication which is regarded as mechanical failure of the micro films.

The surface map analysis of actuator A7 is discussed using the profile plots of the surface maps. Figure 4-23 shows the surface map (a) and the profile plot (b) of the curve up beam of A7. A slice line is drawn to extract the profiles of the curve-up beam. The trench surface is chosen as reference plane ( $\mathrm{y}=0$ ) to measurement the deflection of the beam. The height difference $H_{\max }$ is measured between the horizontal inspector lines of the plot analyzer. 


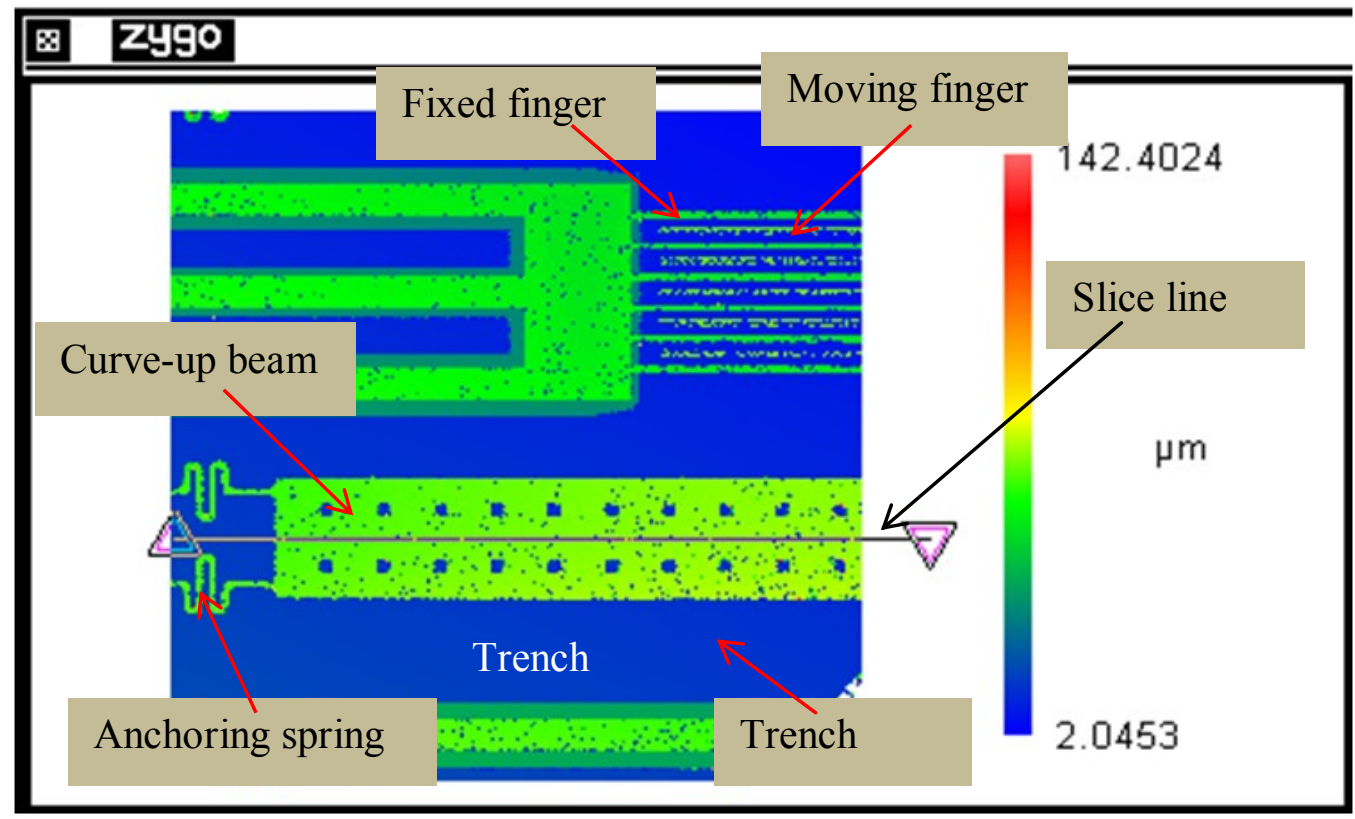

(a)

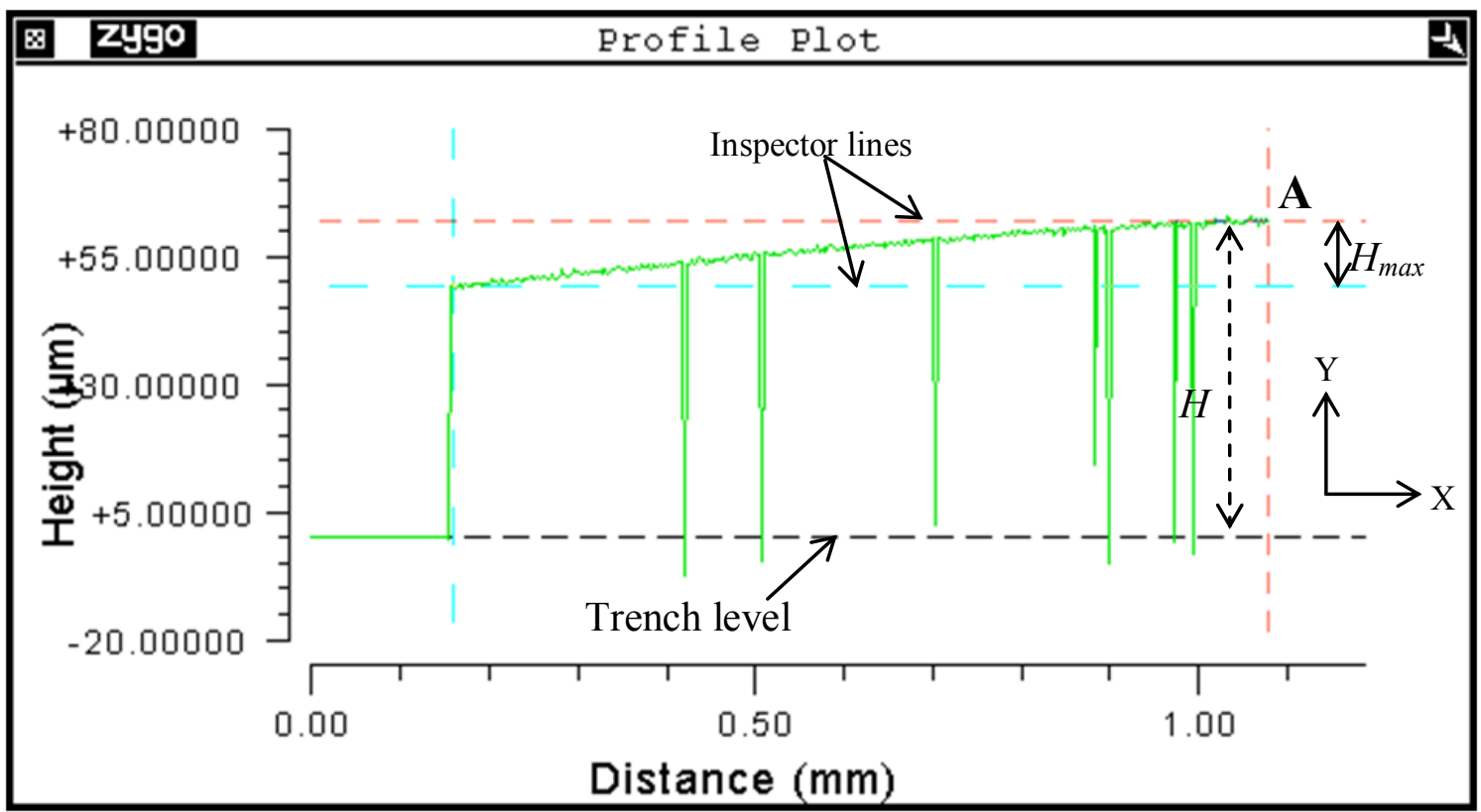

(b)

Figure 4-23 Curve-up beam measurement (a) Surface map (b) profile plot The height of the beam above the trench is $H=63.6 \mu \mathrm{m}$ at point A. The deflection of the curve up beam is calculated by subtracting the thickness of the silicon dioxide, the two silicon nitrides, the 
poly, the sacrificial layer and the thickness of the fixed fingers. Then the deflection of the curve at point $\mathrm{A}$ is $63.6-(25+2+0.35 * 2+0.7+1.1)=13.6 \mu \mathrm{m}$. From the profile plot of Figure $4-23(\mathrm{~b})$ the curve up beam is deflected by $13.6 \mu \mathrm{m}$. Similarly, the height difference between the fixed fingers and the moving fingers were measured. Figure 4-24 (a) shows the magnified section of fingers of the actuator.

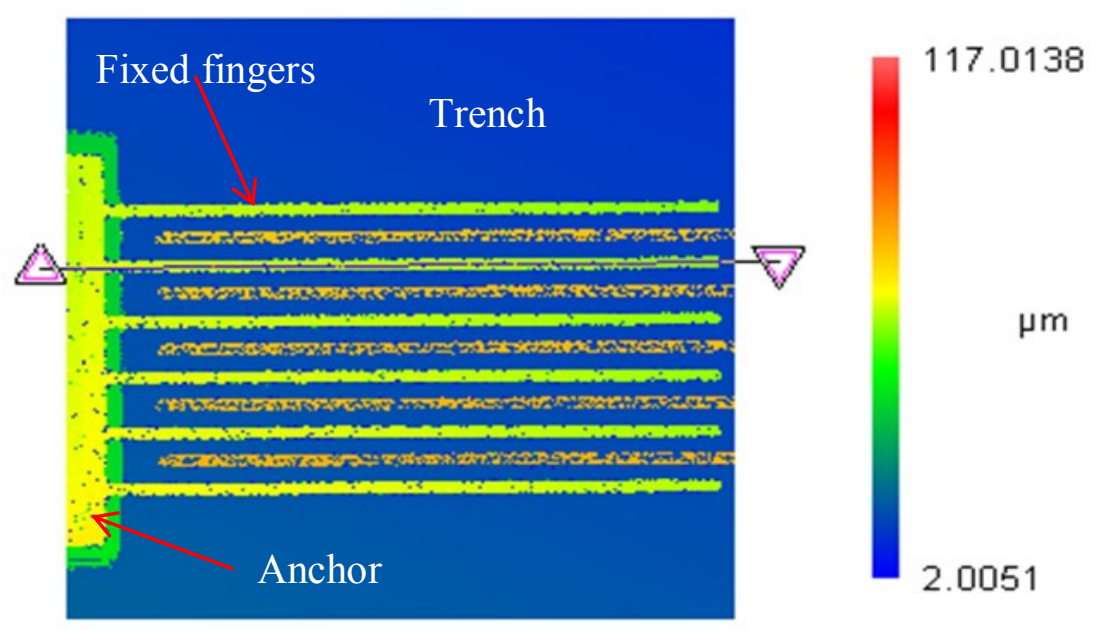

(a)

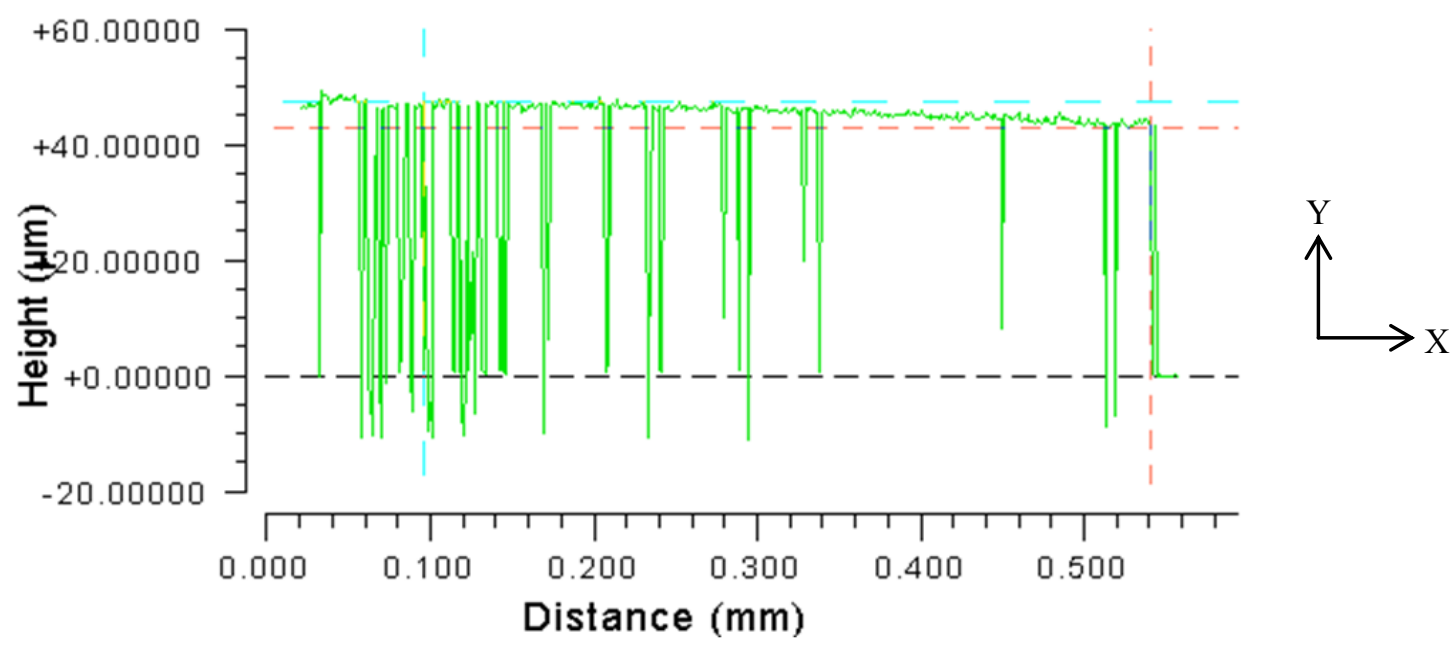

(b)

Figure 4-24 Fixed fingers (a) Surface map and (b) profile plot

The residual stress gradient bent the moving and the fixed fingers. The tips of the fixed fingers bent down by $4.672 \mu \mathrm{m}$ towards the trench as shown in Figure 4-24 (b). 


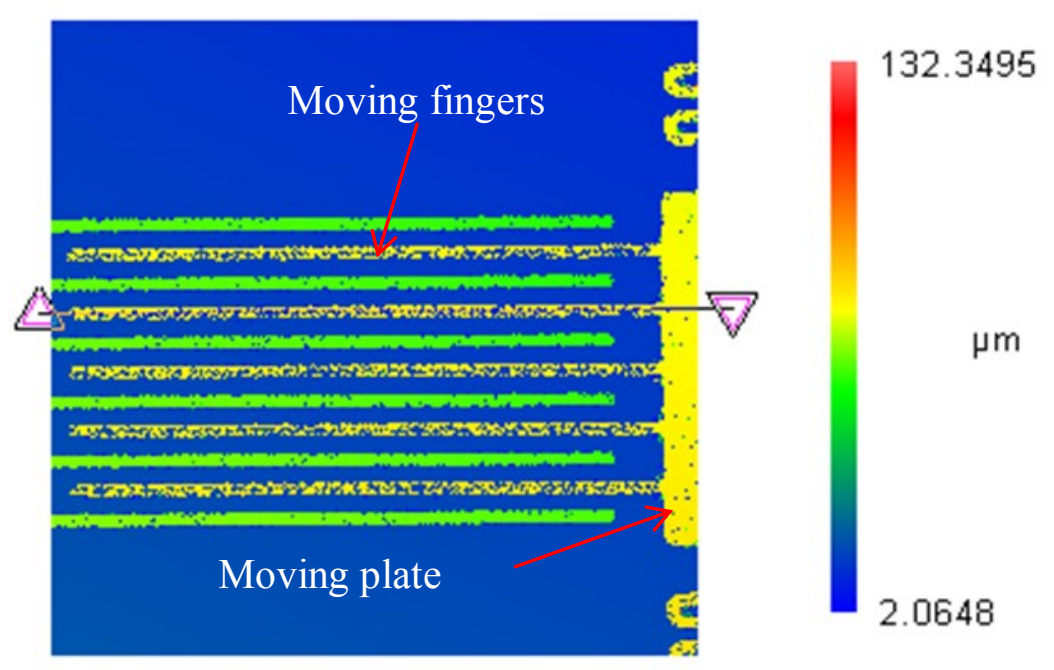

(a)

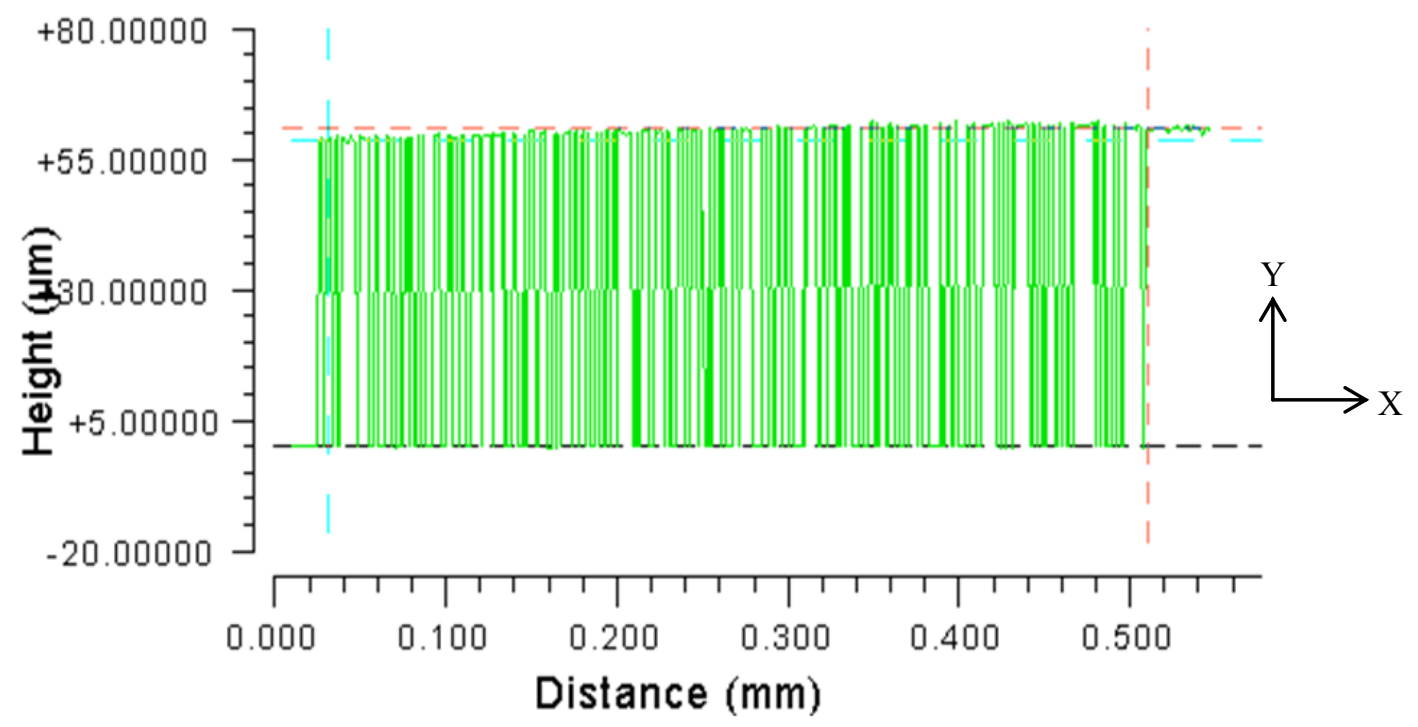

(b

Figure 4-25 Moving fingers (a) Surface map and (b) profile plot

Plots that show height differences between the moving and the fixed fingers are illustrated in Figure 4-26 and Figure 4-27. The free ends of the fingers have large deflection than any point on the fingers. The height difference between the fingers varies along the length of the fingers. The minimum height difference was measured around the middle of the fingers, Figure 4-27 (b), is found to be $15.34 \mu \mathrm{m}$. However, the height differences at the fixed end and the free end of the 
fingers are found to be greater. Based on this investigation the surfaces of the fingers are not planar due to the stress gradient distortion.

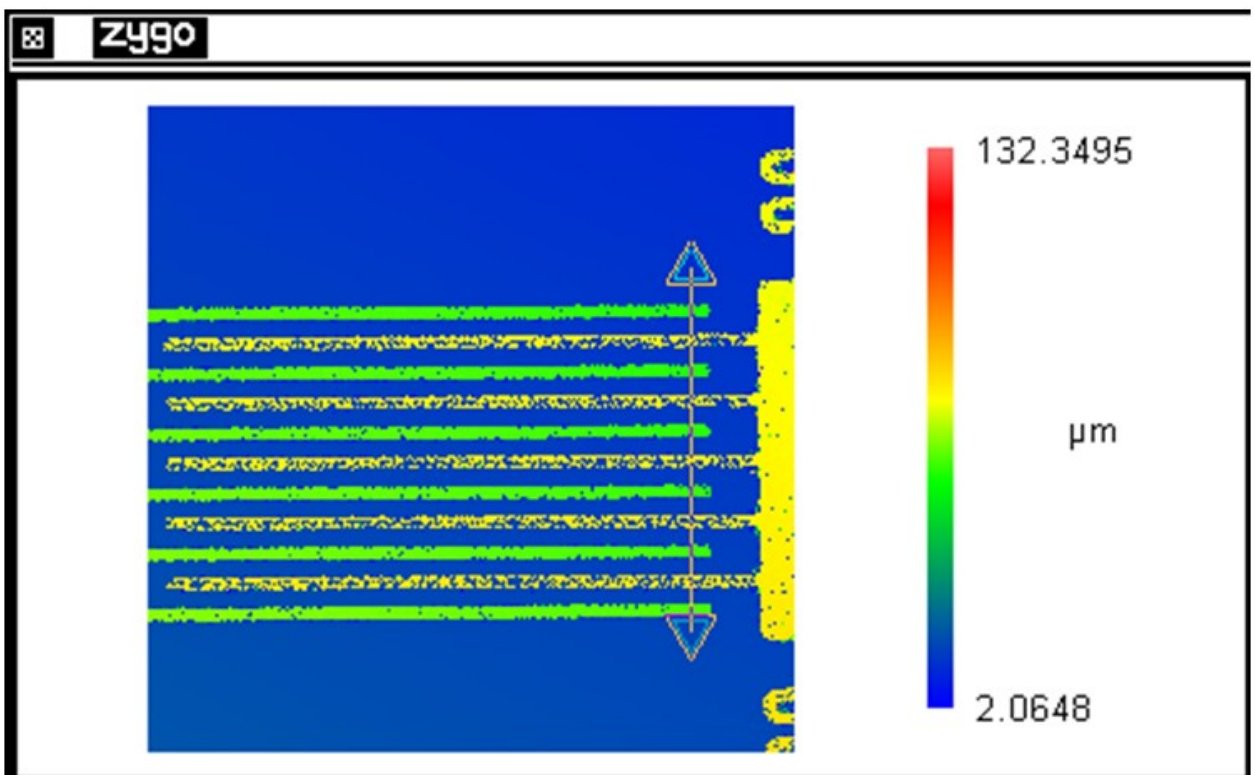

(a)

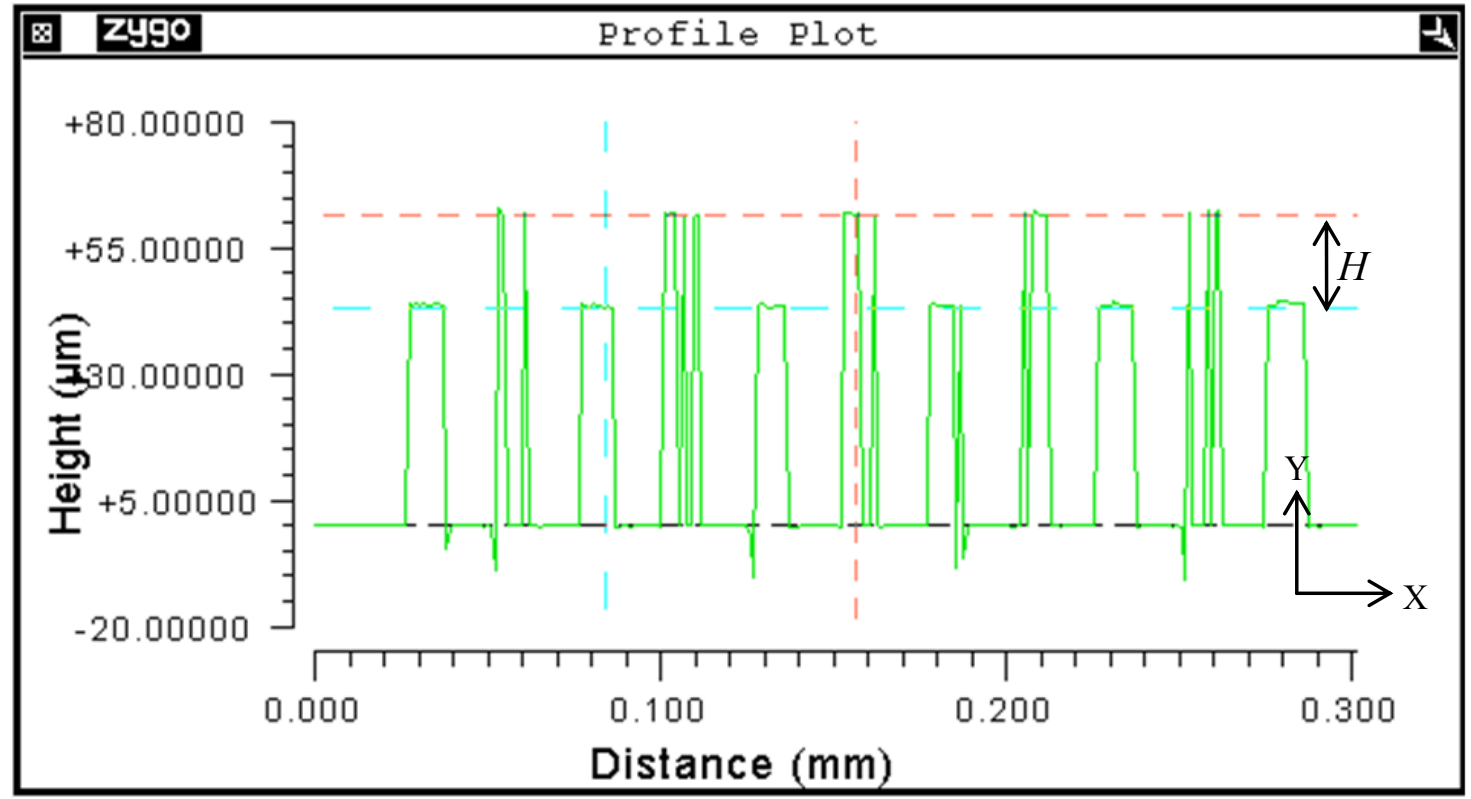

(b)

Figure 4-26 Heights of fingers above trench (a) Moving and fixed fingers (b) profile plot Further measurements on the bending of the moving fingers and fixed fingers are examined. The surface map and profile plots of the moving fingers and the fixed fingers of the Actautor A7 is 
shwon in Figure 4-28 respectively. The measured tip deflection is found to be $2.5 \mu \mathrm{m}$ down towards the substrate trench.
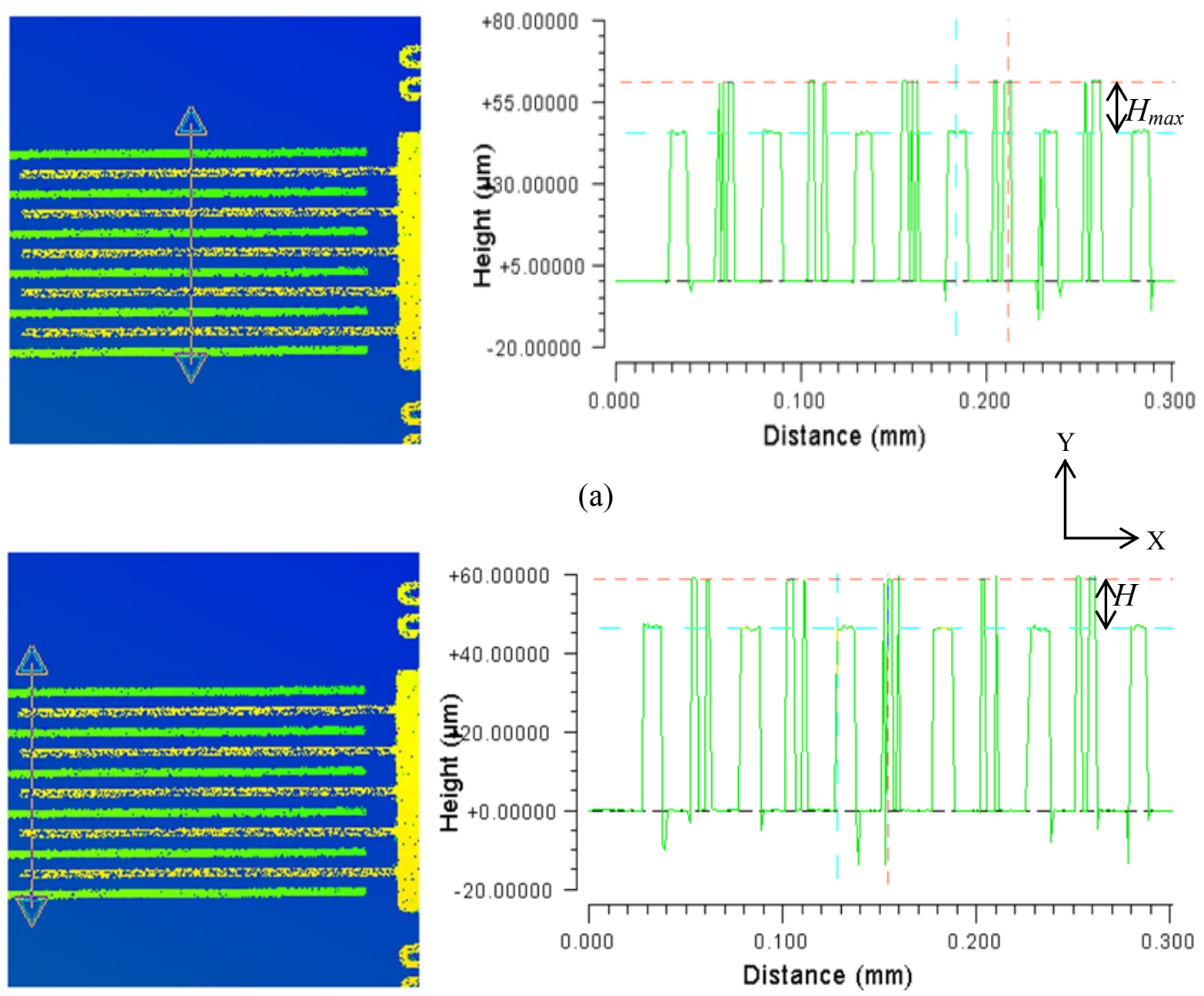

(b)

Figure 4-27 Heights of fingers above the trench (a) Middle section and (b) tip of moving fingers 

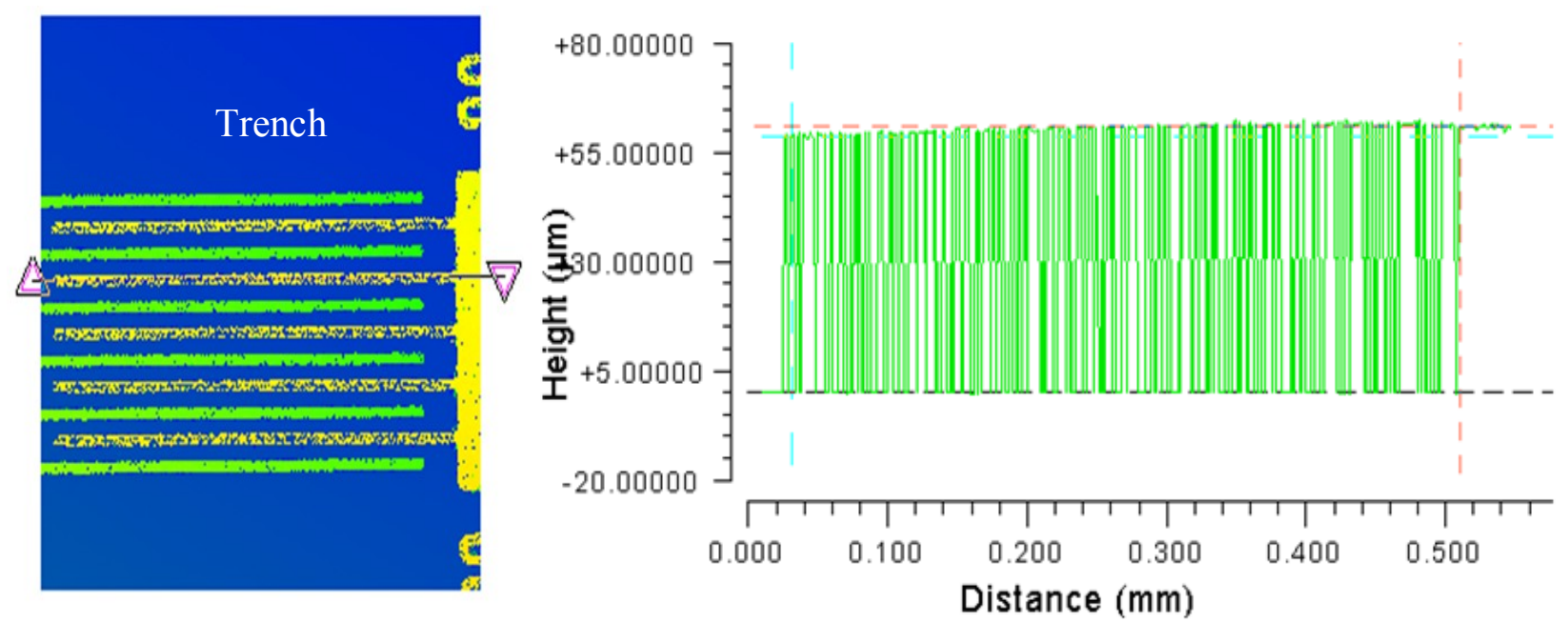

(a)
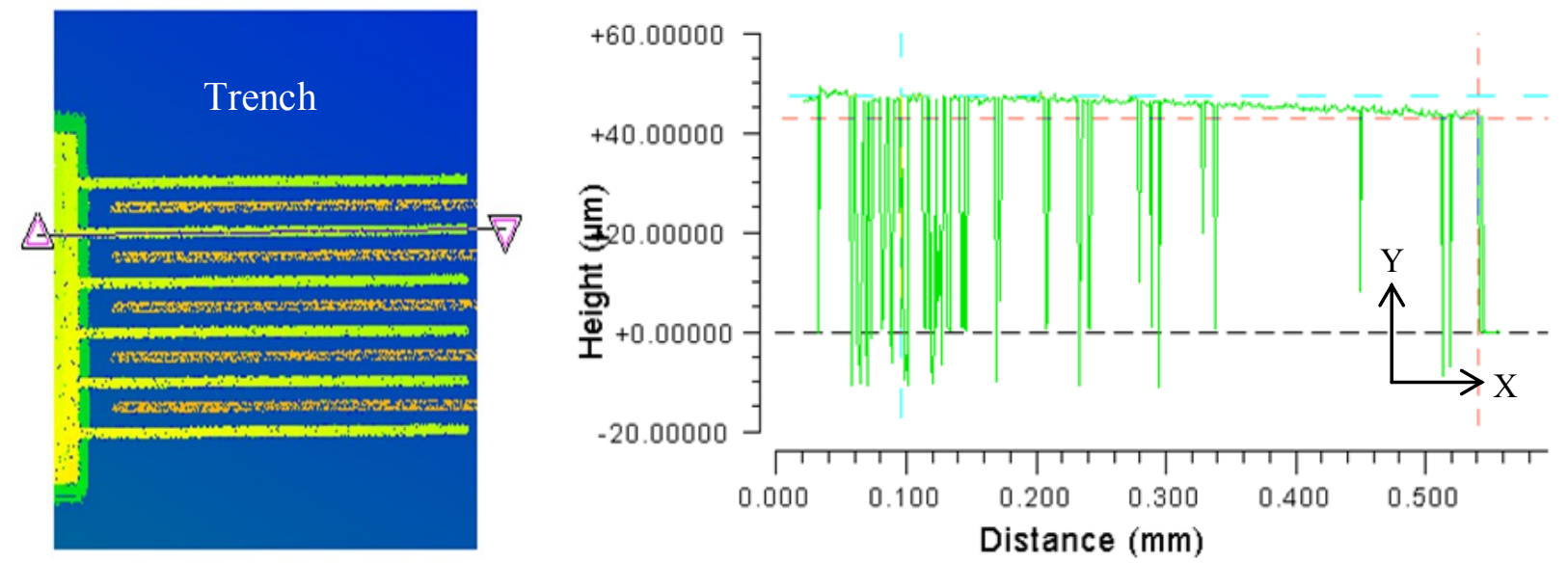

(b)

Figure 4-28 Profile showing the bending of the tips of the (a) moving fingers (b) fixed fingers

All the above statistical analyses are conducted to verify the fabricated prototypes are properly released and the residual stress gradient caused the beams to bend so that the moving fingers are raised above the substrate and the fixed fingers. Therefore, the fabricated prototypes successfully verified the concepts developed and the design principles on which the vertical comb drive actuators rely. 
In conclusion, the no-voltage measurement analysis of the prototypes showed that the residual stress deformed the curve up beams above the substrate. As a result of the deformation of the beams, the moving plates and the moving fingers were raised above the substrate. The novel vertical comb drive actuator now consists of two structures: moving fingers and fixed fingers made of one layer fabrication process.

\subsubsection{Measurement with DC voltage applied}

In this section, the actuators are tested with DC voltages. DC voltage (0-200V) is applied across the bias pads of the actuator to measure the downward displacement of the moving fingers.

Since the actuators were fabricated using a process that has $20 \mu \mathrm{m}$ thick structure with high Young's modulus they require high voltages for driving. The range the DC voltage applied is from $25 \mathrm{~V}$ to $200 \mathrm{~V}$ with $25 \mathrm{~V}$ step.

Two DC probes were used to apply the voltage to the DC pads of the actuators. The fixed fingers are biased while the moving fingers are potentially grounded. When the voltage source is turned on, attractive electrostatic forces pull the moving fingers down and towards the fixed fingers. Therefore, change in the displacements of the moving fingers is obtained by measuring the resulting surface map. When the moving fingers of the actuators move down, the optical profiler is activated to scan and capture the new surface map of the actuator. By drawing the slice line at the desired location on the surface map the profile is plotted. Finally with the inspector lines the distance travelled by the moving plates and the moving fingers are measured. The measured displacements are then compared with the no voltage measurement results to find out the change of the displacement. Mathematically;

$$
\Delta H=H_{\max }-H_{v}
$$

where $H_{\max }$ is the maximum deflection measure at no voltage test, $H_{v}$ the height measured at any actuation voltage, $\mathrm{V}$, and $\Delta H$ is the displacement the moving plate travels.

Actuators A1, A2 and A4-A6 have shown little displacement as the voltages are increased from $25 \mathrm{~V}$ to $200 \mathrm{~V}$. As it was explained in the no voltage tests, these actuators did not achieve sufficient deflection because of the rigidity of the moving structures. At the maximum actuation voltage the generated electrostatic force was not strong enough to pull the moving fingers down. 
Actuator A7 has achieved displacement of $1.5 \mu \mathrm{m}$ displacement at $200 \mathrm{~V}$. The profile plots at $0 \mathrm{~V}$ and 200V are depicted in Figure 4-29 and Figure 4-30, respectively.

\section{Zygo}
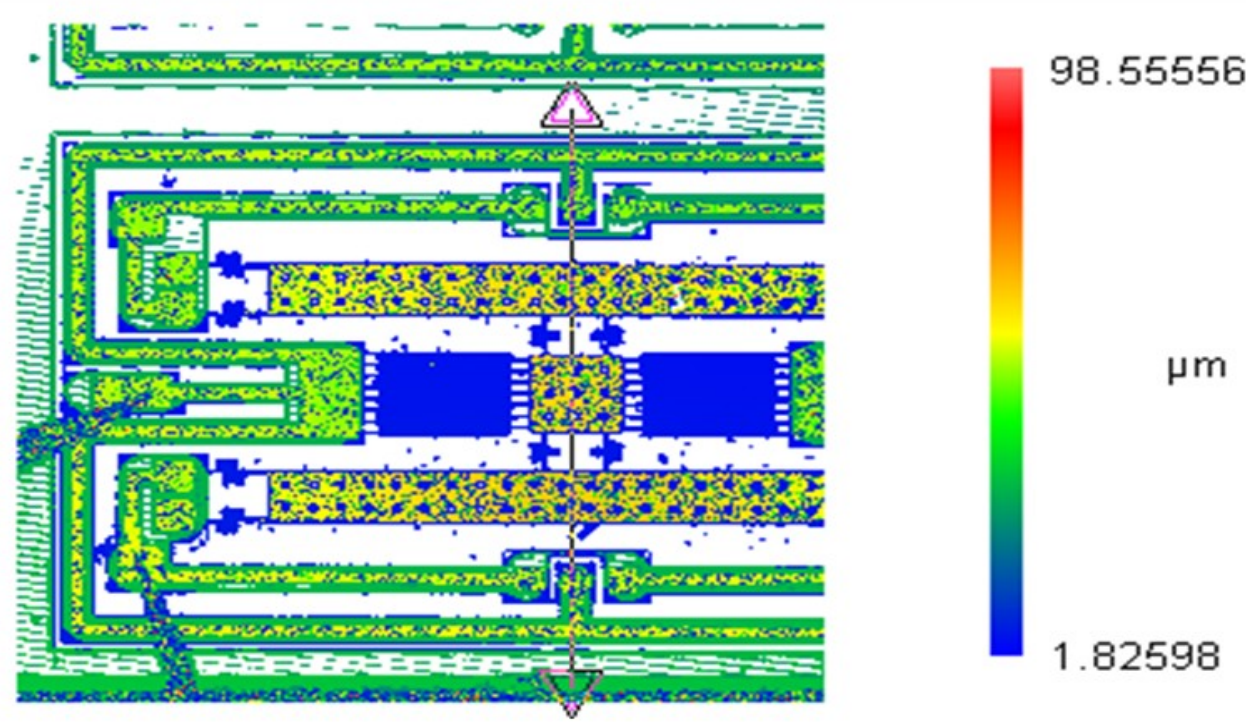

(a)

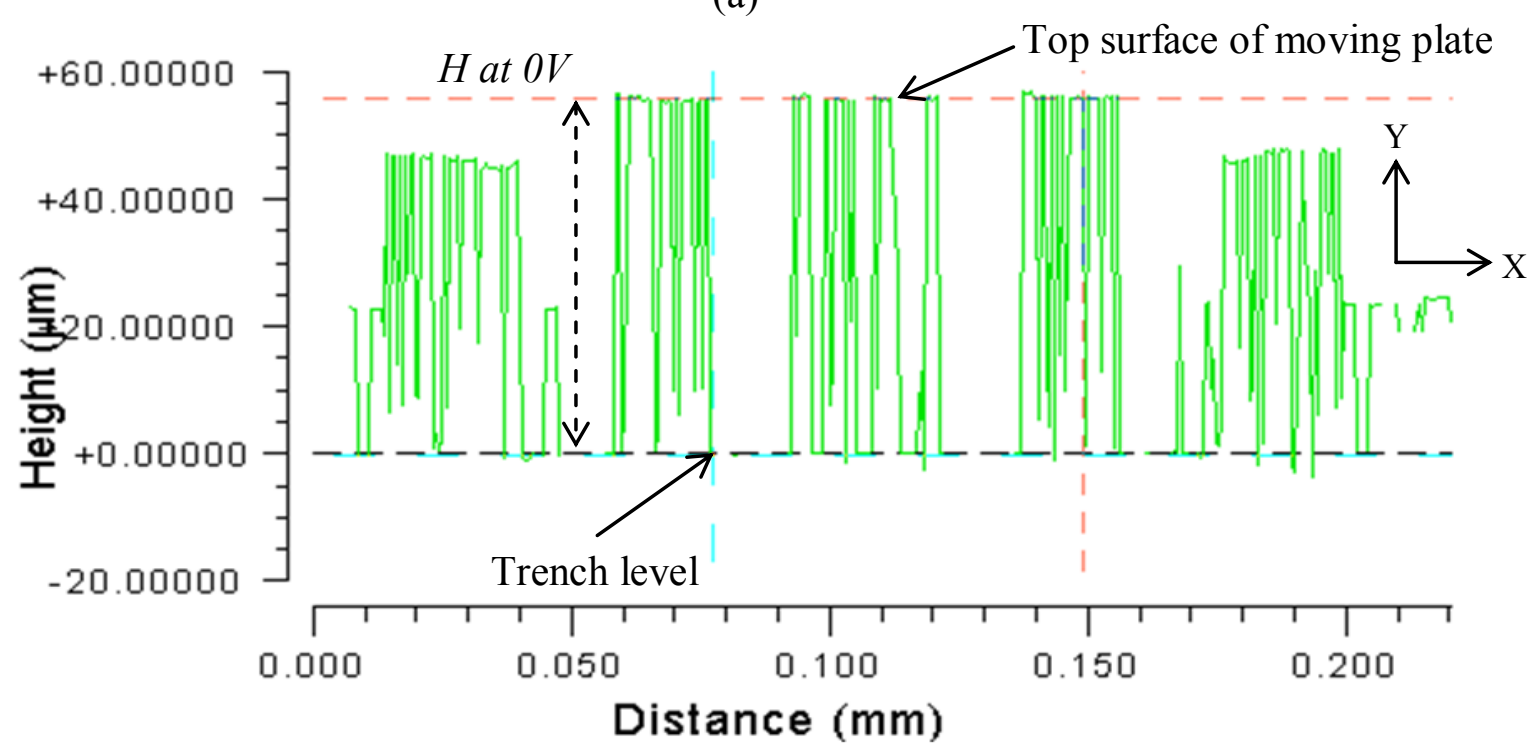

(b)

Figure 4-29 Measurement at 0V (a) Surface map and (b) profile plot 


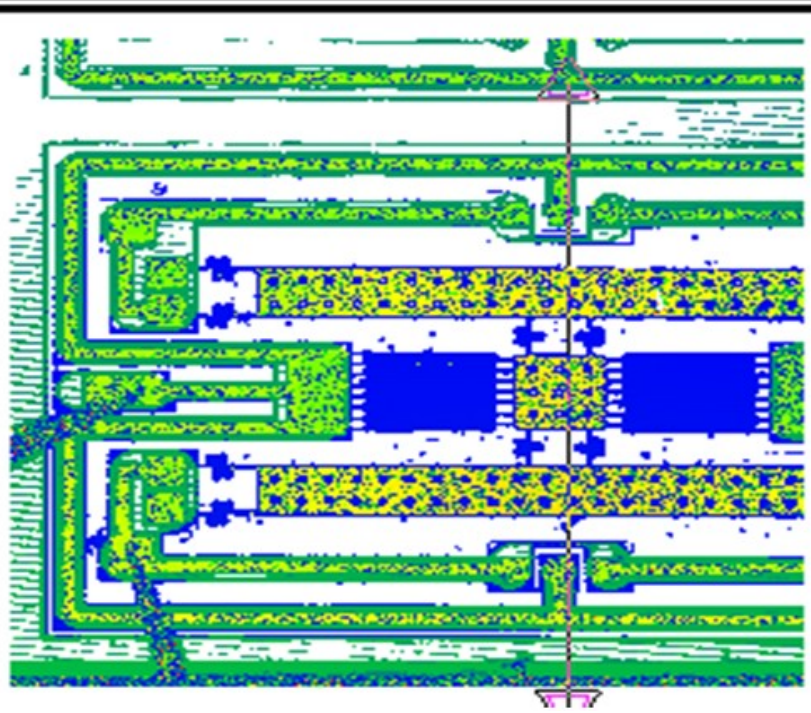

(a)
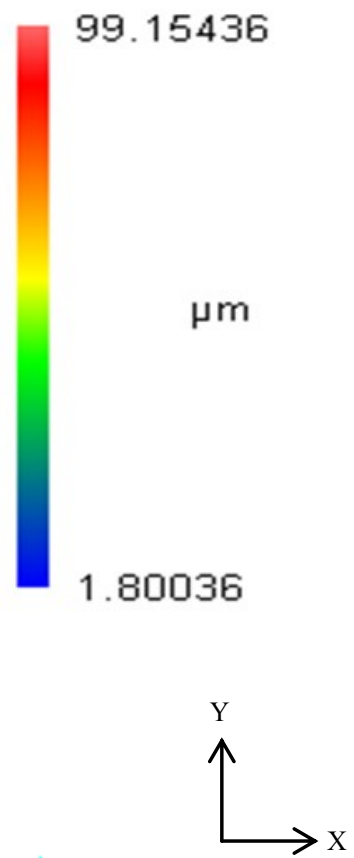

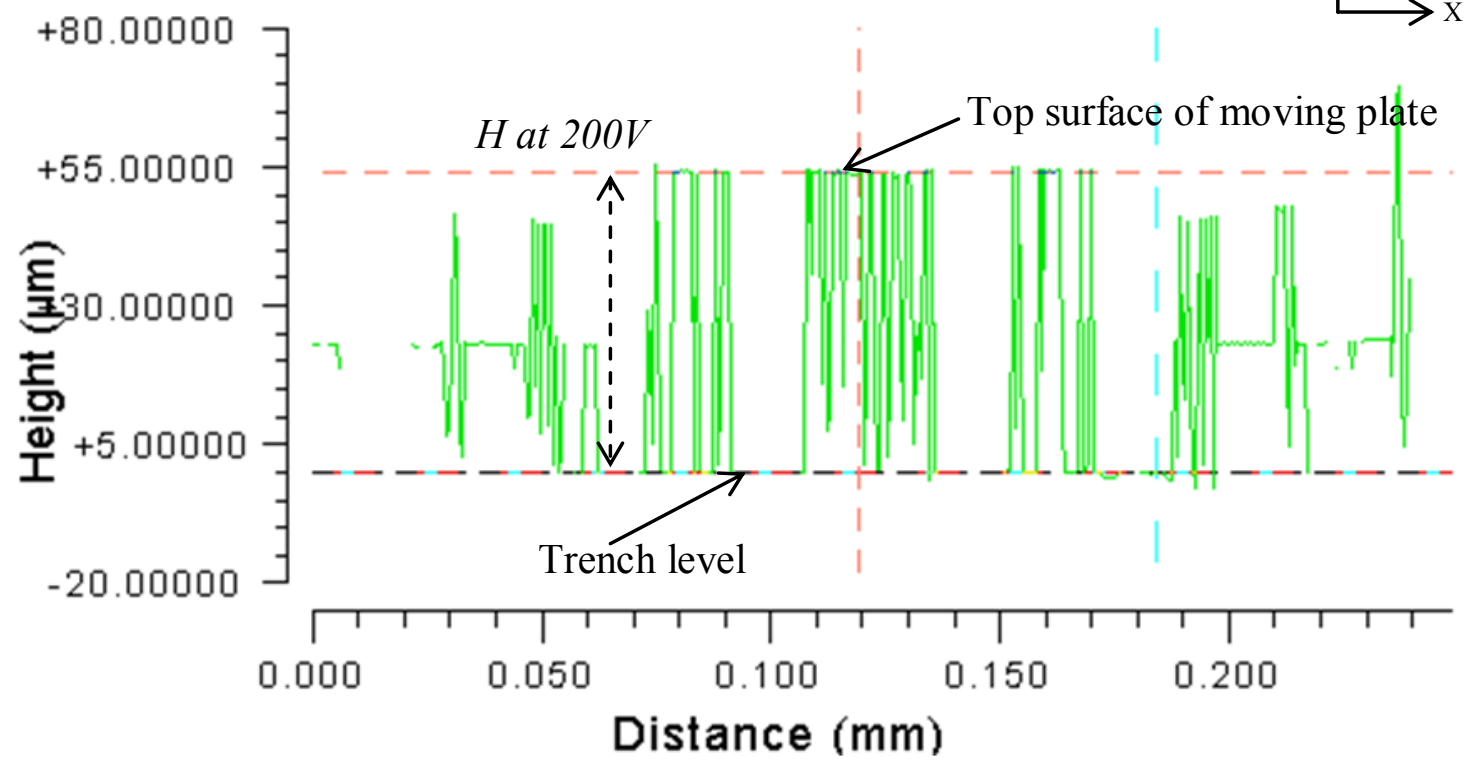

(b)

Figure 4-30 (a) Surface map at 200V and (b) Profile plot at 200V 


\subsection{Result analysis}

The design, fabrication and test of seven actuators have been presented in this chapter. The design and working principle of the actuators was verified by experiments. From the examination and measurements on the prototypes, the curve-up beams are created using the residual stress gradients. The bending of the curve-up beams lifted the moving plate and the moving fingers of the actuators above the substrate. The measured heights difference between the moving fingers and the fixed fingers are shown in Table 4-4. The measured heights were compared with the simulated heights and the deviations were calculated as an error value in percent form. The errors calculated for actuators A1, A2 and A4-A6 exceeded 35\% whereas the A3 and A7 have 15\% and 5\% error, respectively. Figure 4-22 illustrates the Actuator A6 with curve-up beam flat from the SEM micrograph. Based on the experimental analysis, the following conclusions are drawn:

1) The current designs verify the principle of operation of the novel actuator which includes two aspects: the moving fingers are raised after fabrication and the moving fingers move down and toward the fixed fingers when applying a voltage.

2) The problems associated with the actuators developed are:

a) the moving fingers of actuators A1, A2 and A4-A6 are not raised enough;

b) the moving fingers did not move down enough because of small electrostatic attraction force

c) the stiffness of the springs supporting the moving plate are very strong

In general the DC voltage test reveals there is no change in the displacement of A1, A2 and A4A6 (71\% of the actuator designs). The actuators A3 and A5 (29\% of the actuator designs) did not achieve higher displacement under voltage test. Hence, the actuators fabricated do not produce high displacement.

The solutions to overcome the problems of the current designs are:

- reducing the stiffness of the anchoring springs of the curve-up beam

- increasing the number of fingers to increase the attraction force

- lifting the moving finger to a large height above the substrate

- including a separate DC actuation electrode to drive the moving plate

- using flexible moving plate supporting springs 
- reducing the gaps between the moving fingers and the fixed fingers

\subsection{Conclusion}

A vertical comb-drive MEMS actuator was fabricated using single metal layer by forming two structures: moving and fixed. CoventorWare based simulation and analysis have been conducted to characterize the necessary deformations of the clamped-clamped curve-up beams that lift the moving plates/fingers of the actuator using residual stress gradient on the nickel of the MetalMUMPs fabrication process. Two kinds of springs: rectangular and serpentine were used to determine the out of plane deformation of the curved beams. The dependence of the deflections of the curved beams on the length and the type of the spring used is studied. The curve-up beams anchored with serpentine springs achieved large deflection compared with the beams with rectangular spring. A curve fitting tool of Matlab was used to fit the simulated deformations of the beams to find equation that relates length of the unstressed beam to the deflection of the beams. The curved beams were used to raise the moving fingers and the moving plate of the actuator above the substrate.

The prototypes of the actuators were fabricated using the MetalMUMPs process. The fabricated prototypes were inspected using Zygo new view $6 \mathrm{~K}$ microscope. The surface map and profile plots were analyzed using measurements. The design principle of the actuators was verified with measurements of the moving fingers and the curve-up beams which were lifted above the substrate due to the residual stress gradient. However, the moving fingers and the curve-up beams of actuators A1, A2 and A4-A6 were not sufficiently raised above the substrate. Hence, these designs required high voltage to move the moving fingers down towards the fixed fingers.

On the other hand, actuators A3 and A7, curve-up beams and moving fingers are bent out of plane and upward by $10 \mu \mathrm{m}$ and $15 \mu \mathrm{m}$, respectively, above the substrates were lifted above the substrate due to the residual stress gradient's bending moment.

DC voltage tests were also conducted to verify the working principle. For actuator A7 at 200V the moving fingers achieved a displacement of $1.5 \mu \mathrm{m}$ was obtained.

Analyses of the experiments indicate that further design improvements are necessary for large displacements. The possible solution is to reduce the stiffness of the curved beams so that large 
out of plane deformation can be obtained. Increasing the number of fingers increases the electrostatic force of attraction so that the travel range of the moving fingers becomes large. A driving electrode under the moving plate of the actuator can also enhance the travel range of the fingers by increasing the force at a given voltage. The improved design of the actuator is discussed together with application to RF MEMS tunable capacitors in Chapter 5. 


\section{Application of the Vertical Comb Actuator to MEMS Tunable Capacitor}

\subsection{Introduction}

This chapter discusses the design, fabrication and performance testing of tunable capacitors developed using an improved design vertical comb drive actuators developed in Chapter 4. The first objective is focused at redesigning of the actuator to get large deflection. High voltages were required to drive the actuators and the electrostatic forces generated were not strong enough to pull the moving fingers. Most of the curve-up beams of the actuators did not reach the predicted deflections. Another reason for improving the designs is that the actuators were stiff because of non-uniform deposition in the MetalMUMPs fabrication process.

The major improvements include modifying the geometry of both the curve up beams and the actuator parts. The modification on the curve up beam targets reduced stiffness so that higher deflection will be achieved. These are done by reducing stiffness of the anchoring springs and using thinner long curve up beams.

On the other hand, the modification of the actuator increases the travel range of the moving fingers and reduces the voltages required. This is done by increasing the number of moving fingers and the fixed fingers, narrowing the gap between adjacent fingers, and lowering stiffness of serpentine springs by which the moving plate is suspended.

Simulations are conducted to determine the deflections of the curve-up beams and predict the maximum height difference between the moving and the fixed fingers after fabrication.

Prototypes of the tunable capacitors are fabricated and the electromechanical performance measurements over voltages of 0-200V are presented. RF performance test results are discussed using S-parameter measurements. The S-parameters are used to determine substrate effects, such as parasitic capacitance and impedances at microwave frequency which are difficult to determine quantitatively by simulations or analytical methods. Quality factors of the tunable capacitors are extracted from the S-parameters. Power losses are also computed using the S-parameter results to account of the RF power dissipated in the substrate and the metal. 


\subsection{Design of vertical comb drive tunable capacitor}

The section discusses the redesigning of the curve up beams to achieve high deflection. The design of tunable capacitor using the new curve-up beam and the vertical comb-drive actuators is presented.

\subsubsection{Redesign of curve up beam}

The new clamped-clamped curve up beams used for the design of the tunable capacitors is shown in Figure 5-1. The shapes of the non-deformed and deformed beams are shown. A box spring (Figure 5-2) is used for anchoring the beams to the substrate. The improvements made are intended to achieve higher deflections of the curve-up beams and to raise the moving fingers to a larger deflection above the substrate. The first improvement to get larger deflection is to use longer beams with narrow width compared to the designs in Chapter 4.
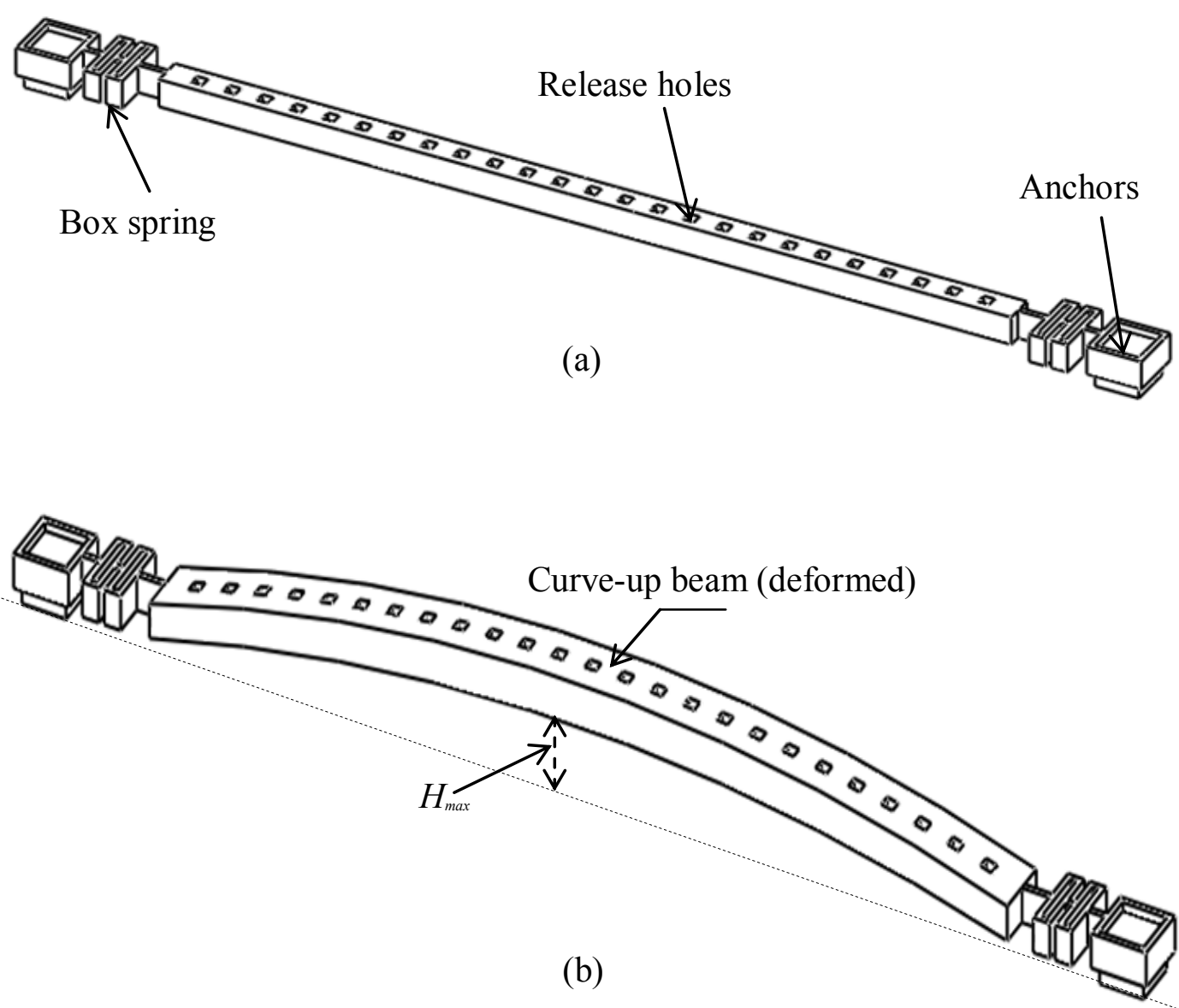

Figure 5-1 Clamped-clamped (a) non-deformed and (b) deformed gradient (curve-up beam) 


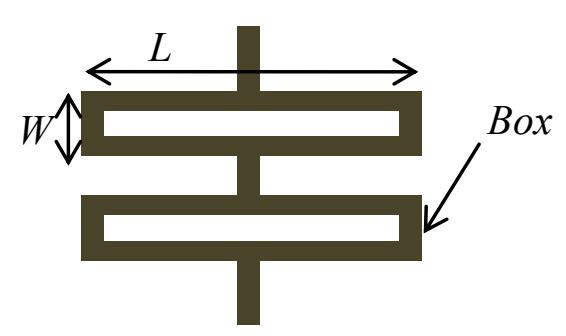

Figure 5-2 Box spring

\subsubsection{Capacitor layout}

The complete layout of the tunable capacitor is shown in Figure 5-3 together with label on its parts. The important changes to the actuator besides the curve up beam, include large number of fingers and flexible serpentine springs (Figure 5-4) to suspend the moving plate. The electrostatic force increases with the number of fingers while the serpentine springs provide low stiffness. The dimensions of the capacitors including the curve-up beams are listed in Table 5-2.

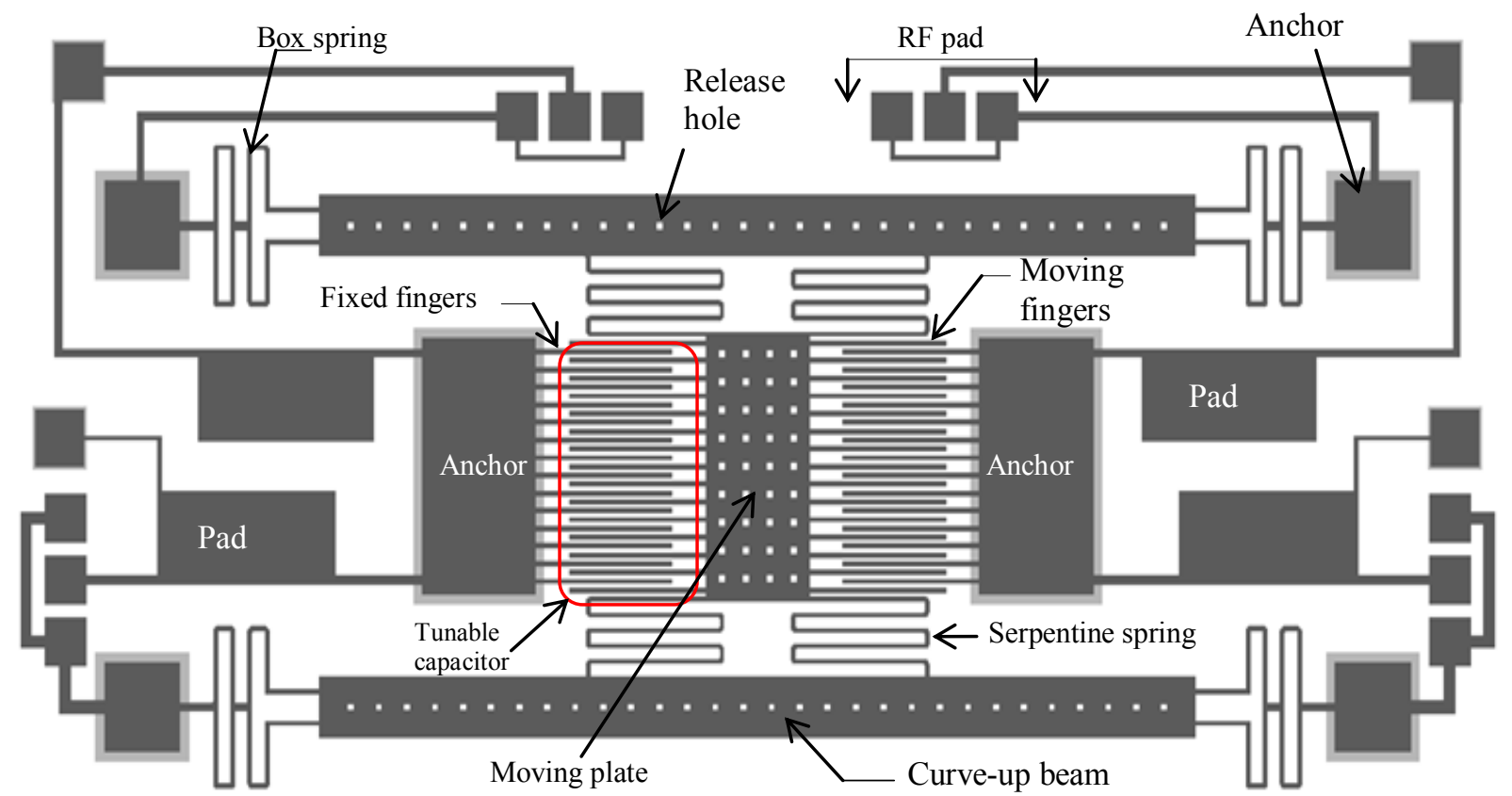

Figure 5-3 Tunable capacitor layout

After release step of the fabrication process, the fixed and the moving fingers are vertically separated due to the residual stress gradient on the nickel layer, as explained in Section 4.5. 
Serpentine springs are added to support the moving plate of the capacitor. The end-to-end lengths ( $\mathrm{L}$ and $\mathrm{W}$ ) and the number of turns of the serpentine springs are increased to lower the spring constant. The schematic of serpentine springs used in the chapter are shown in Figure 5-4.
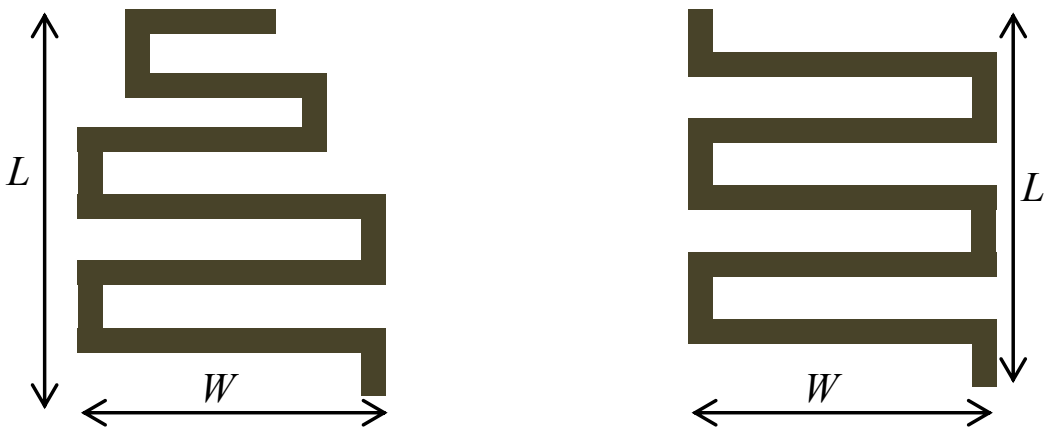

Figure 5-4 Two serpentine springs

Poly-silicon electrodes are added under the moving plates of the actuator as a second actuator to reduce the voltage required to bring the moving fingers down towards the fixed fingers. The dimensions of the moving plate are selected in such a way as to avoid the deformation caused by residual stress gradient. CoventorWare simulations were used to analyze the effect of the residual stress gradient on plate. The dimensions of the plate are finally selected based on the possible minimum deformation. The bending of the moving plate lowers the maximum height difference between the moving and the fixed fingers.

The number of fingers that could be put on the moving plate is thus dependent on the length of the moving plate. The minimum feature size requirements the MetalMUMPs [103] was used to select the appropriate gap between the moving and the fixed fingers.

\subsection{Simulation of curve up beams}

The residual stress gradient is applied to simulate the deformation of the new curve up beams. In the simulation the material properties database in the Coventor process foundry is used. The Young's modulus of nickel in the database is $159 \mathrm{GPa}$ and the stress gradient is $-4.72 \mathrm{MPa} / \mu \mathrm{m}$ [101].

Even though the metal layer consists of a $0.5 \mu \mathrm{m}$ thin coating gold film, its effect is negligible. The nickel's residual stress gradient is the only dominant parameter that cause significant deformation compared with the gold. The deformations of ten curved-up beams anchored with 
box springs are analyzed. The dimension of the curve up beams and the box springs are listed in Table 5-1. The maximum out of plane deflections reached by the beams are also shown in the same table.

Table 5-1 Dimensions of curve-up beams and deflections

\begin{tabular}{cccc}
\hline $\begin{array}{c}\text { Curve-up } \\
\text { beam }\end{array}$ & $\begin{array}{c}\text { Initial dimensions } \\
(\mathrm{L} / \mathrm{W} \text { in } \mu \mathrm{m})\end{array}$ & $\begin{array}{c}\text { Box-spring } \\
(\mathrm{L} / \mathrm{W} \text { in } \mu \mathrm{m})\end{array}$ & Deflection $(\mu \mathrm{m})$ \\
\hline C1 & $2000 / 50$ & $408 / 400$ & 12.89 \\
C2 & $2500 / 50$ & $408 / 400$ & 18.36 \\
C3 & $2000 / 50$ & $408 / 400$ & 12.89 \\
C4 & $2500 / 100$ & $408 / 400$ & 19.73 \\
C5 & $2500 / 100$ & $408 / 400$ & 19.73 \\
C6 & $2558 / 150$ & $410 / 402$ & 20.82 \\
C7 & $2558 / 150$ & $408 / 410$ & 21.17 \\
C8 & $2500 / 50$ & $408 / 400$ & 18.36 \\
C9 & $2100 / 100$ & $408 / 400$ & 14.84 \\
C10 & $2000 / 100$ & $408 / 400$ & 13.71 \\
\hline
\end{tabular}

Notation: $\mathrm{L}=$ length and $\mathrm{W}=$ width

The deformations of the curve-up beams are shown in Figure 5-5 (a) to (f). It is found that thinner and longer curve up beams attain higher deflection for the same size of anchoring spring. Beams $\mathrm{C} 6$ and $\mathrm{C} 7$ have attained larger deflection. 


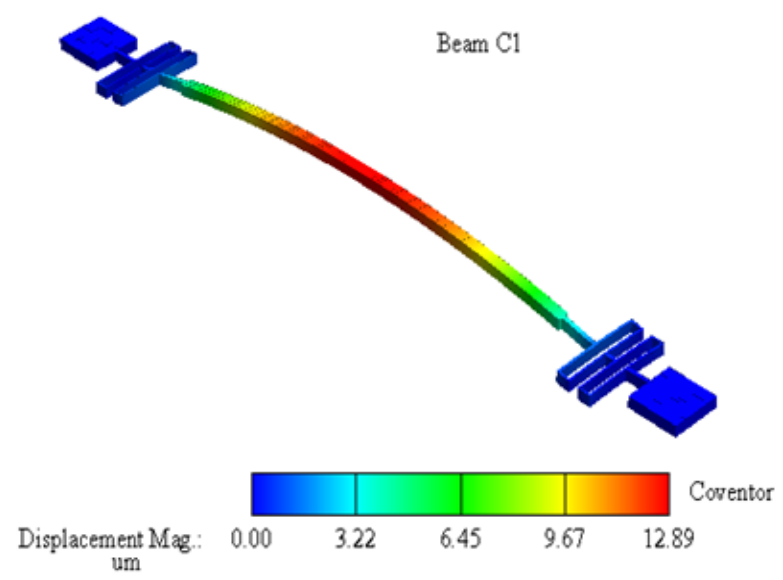

(a)

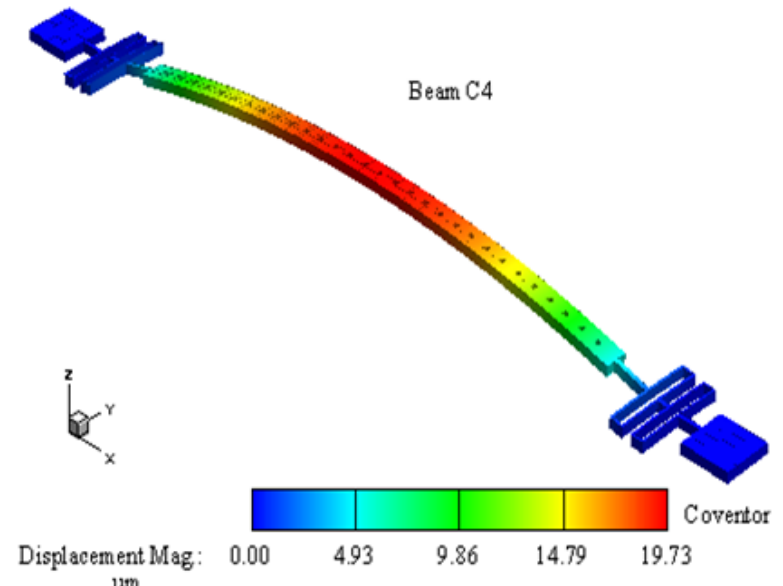

(c)

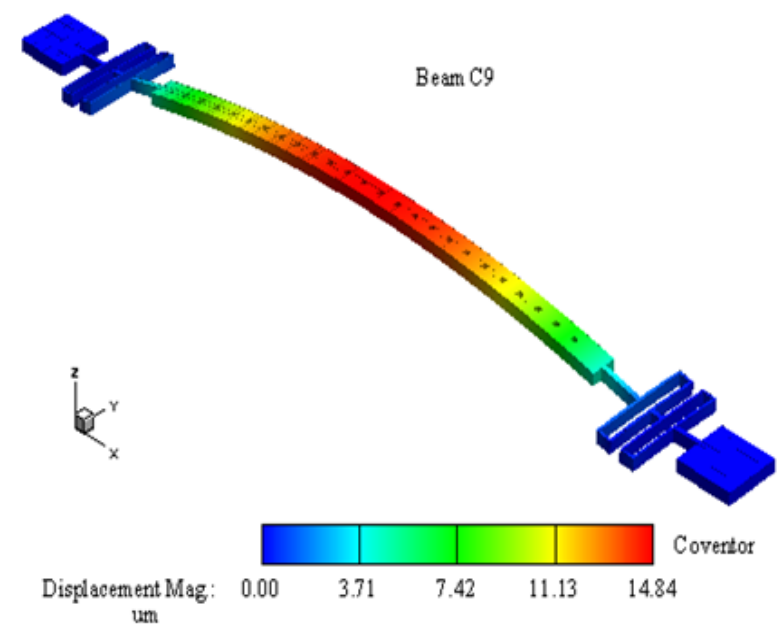

(e)

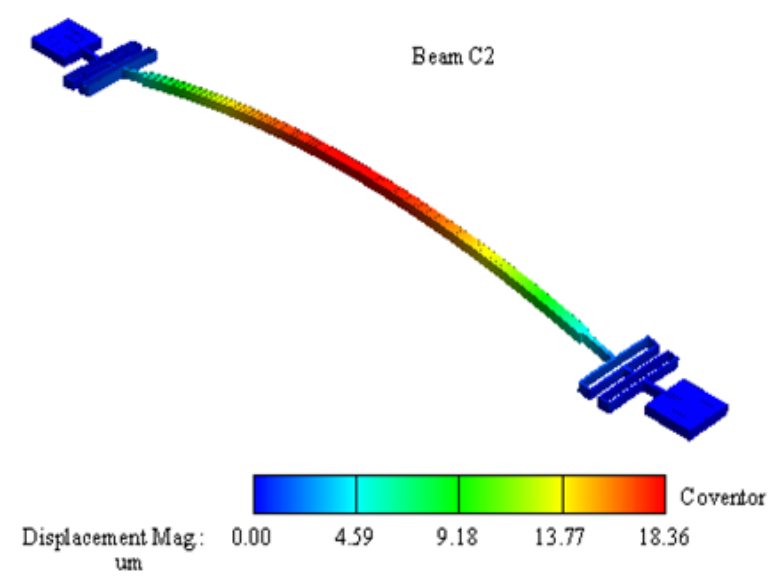

(b)

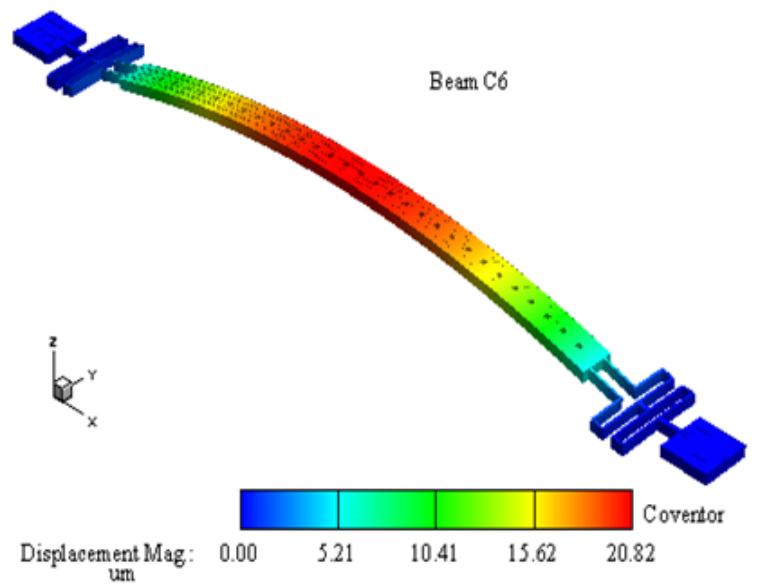

(d)

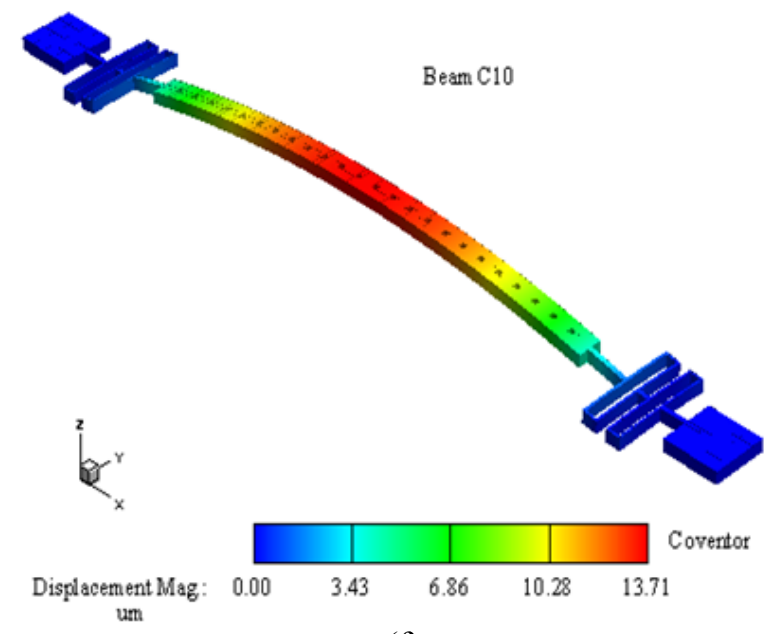

(f)

Figure 5-5 Simulated deformation (displacements) of Curve-up beam C1-C10 


\subsection{Capacitor 3D models and simulations}

In this section, the $3 \mathrm{D}$ model of the capacitors is built from the $2 \mathrm{D}$ layouts. The dimensions of the capacitors are listed in Table 5-2. The symbols L and W stand for the length and the width of structures of the capacitors. The notation $C i$ is used for the name of the capacitor design with $i=1,2,3, \ldots, 10$. The 3D models of the capacitors and the substrate are shown in Figure 5-6.

Table 5-2 Geometric parameters of tunable capacitor

\begin{tabular}{|c|c|c|c|c|c|c|c|c|}
\hline \multicolumn{2}{|c|}{$\begin{array}{l}\text { Capacitor } \\
\qquad C i\end{array}$} & $\begin{array}{l}\text { Moving } \\
\text { fingers }\end{array}$ & $\begin{array}{l}\text { Fixed } \\
\text { fingers }\end{array}$ & $\begin{array}{c}\text { Curve-up } \\
\text { Beam }\end{array}$ & Box spring & $\begin{array}{c}\text { Serpentine } \\
\text { spring }\end{array}$ & $\begin{array}{c}\text { Moving } \\
\text { plate }\end{array}$ & $\begin{array}{l}\text { Gap b/n } \\
\text { fingers }\end{array}$ \\
\hline \multirow[b]{2}{*}{$\mathrm{C} 1$} & $\mathrm{~L}$ & 250 & 250 & 2000 & 408 & 250 & 480 & 10 \\
\hline & $\mathrm{W}$ & 9 & 9 & 50 & 400 & 400 & 210 & \\
\hline \multirow[b]{2}{*}{$\mathrm{C} 2$} & $\mathrm{~L}$ & 350 & 400 & 2500 & 408 & 250 & 494 & 12 \\
\hline & $\mathrm{W}$ & 10 & 10 & 50 & 400 & 400 & 200 & \\
\hline \multirow[b]{2}{*}{$\mathrm{C} 3$} & $\mathrm{~L}$ & 350 & 350 & 2000 & 408 & 240 & 683 & 15 \\
\hline & W & 9 & 9 & 50 & 400 & 400 & 200 & \\
\hline \multirow[b]{2}{*}{ C4 } & L & 400 & 440 & 2500 & 408 & 250 & 667 & 9 \\
\hline & $\bar{W}$ & 9 & 9 & 100 & 400 & 400 & 200 & \\
\hline \multirow[b]{2}{*}{ C5 } & $\mathrm{L}$ & 350 & 410 & 2500 & 408 & 249 & 451 & 9 \\
\hline & W & 9 & 9 & 100 & 400 & 400 & 200 & \\
\hline \multirow{2}{*}{$\begin{array}{c}* \\
\text { C6 }\end{array}$} & $\mathrm{L}$ & 400 & 400 & 2558 & 410 & 199 & 670 & 12 \\
\hline & $\mathrm{W}$ & 10 & 10 & 150 & 402 & 400 & 300 & \\
\hline \multirow{2}{*}{$\begin{array}{l}* * \\
\mathrm{C} 7\end{array}$} & $\mathrm{~L}$ & 400 & 400 & 2558 & 408 & 199 & 670 & 12 \\
\hline & $\bar{W}$ & 10 & 10 & 150 & 410 & 400 & 300 & \\
\hline \multirow[b]{2}{*}{ C8 } & $\mathrm{L}$ & 250 & 250 & 2500 & 408 & 250 & 494 & 12 \\
\hline & W & 10 & 10 & 50 & 400 & 400 & 200 & \\
\hline \multirow[b]{2}{*}{ C9 } & $\mathrm{L}$ & 350 & 350 & 2100 & 408 & 249 & 451 & 9 \\
\hline & $\mathrm{W}$ & 9 & 9 & 100 & 400 & 400 & 200 & \\
\hline \multirow[b]{2}{*}{ C10 } & $\mathrm{L}$ & 350 & 400 & 2000 & 408 & 250 & 670 & 12 \\
\hline & $\mathrm{W}$ & 10 & 10 & 100 & 400 & 400 & 200 & \\
\hline
\end{tabular}




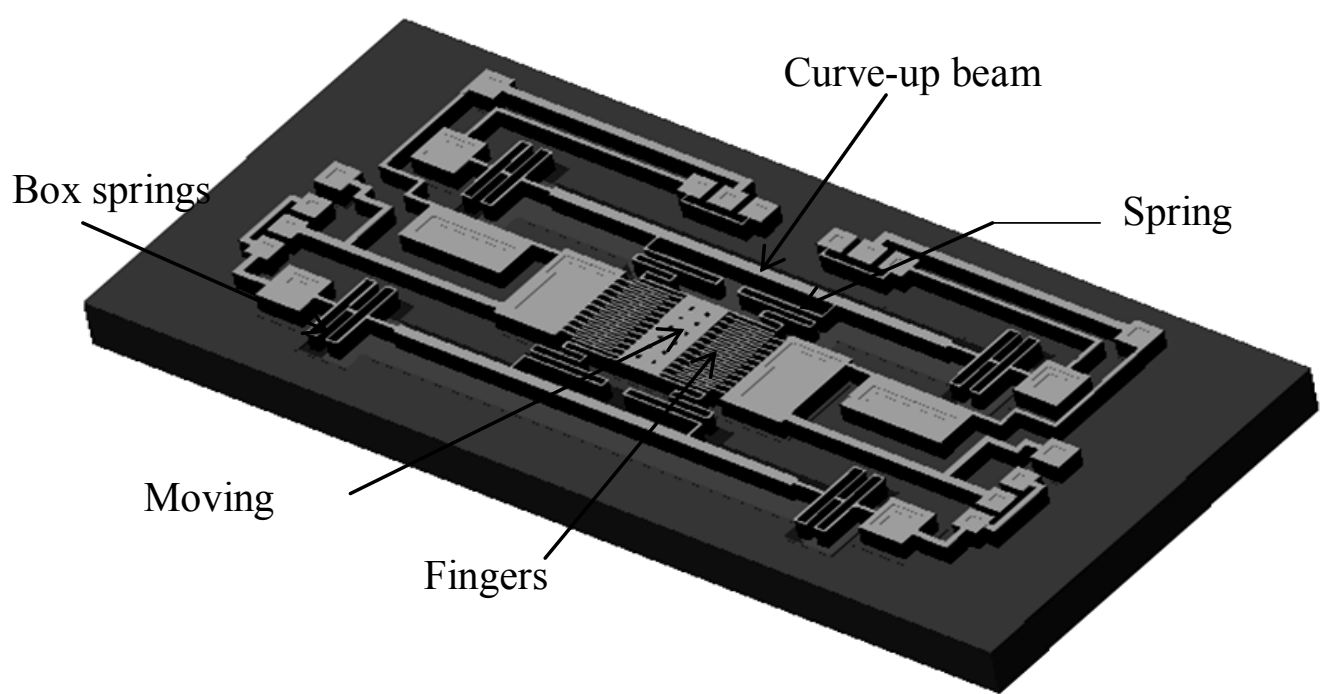

(a)

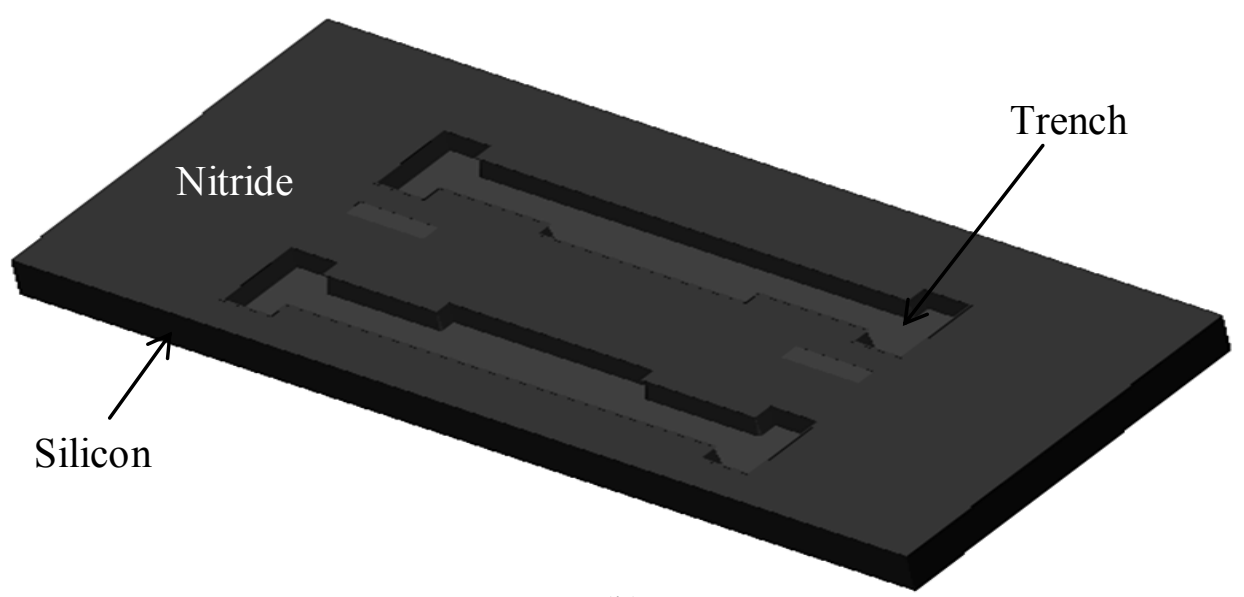

(b)

Figure 5-6 3D view of tunable capacitor C1

Simulations of the capacitors are performed using CoventorWare solver. The vertical height difference between the moving and the fixed fingers, denoted by $H_{\max }$, represents the maximum height from the top surface of the moving fingers to the top surface of the fixed fingers. It is the expected height the moving fingers and the moving plate of the capacitor achieve after fabrication.

The deformations of the capacitors are shown in Figure 5-7 (a), (b) and (c) for capacitors C1, C5 and $\mathrm{C} 7$ respectively. The deformations of $\mathrm{C} 2-\mathrm{C} 4, \mathrm{C} 6$ and $\mathrm{C} 7-\mathrm{C} 10$ are shown in Appendix B. From the simulated deformation, the moving plates have relatively larger deflection compared to 
the curve-up beams. This is because the stress gradient induced high bending moment on the moving fingers, moving plates and the plate supporting springs. When longer fingers are used the bending moment is further high on the fingers and tends to create large vertical separation between the moving plates and the fixed fingers. However, in order to prevent the downward bending of the moving fingers, the lengths of the moving fingers are selected to keep the top surface of the moving plates and the moving fingers planar. Table 5-3 shows the maximum heights obtained after the moving fingers and the moving plate are added to the curve-up beams.

Table 5-3 Simulated maximum height $\left(H_{\max }\right)$ of capacitors

\begin{tabular}{lllllllllll}
\hline Capacitor & $\mathrm{C} 1$ & $\mathrm{C} 2$ & $\mathrm{C} 3$ & $\mathrm{C} 4$ & $\mathrm{C} 5$ & $\mathrm{C} 6$ & $\mathrm{C} 7$ & $\mathrm{C} 8$ & $\mathrm{C} 9$ & $\mathrm{C} 10$ \\
\hline$H_{\max }(\mu \mathrm{m})$ & 14.02 & 19.79 & 14.65 & 21.73 & 20.83 & 22.34 & 22.66 & 19.79 & 15.93 & 15.72 \\
\hline
\end{tabular}

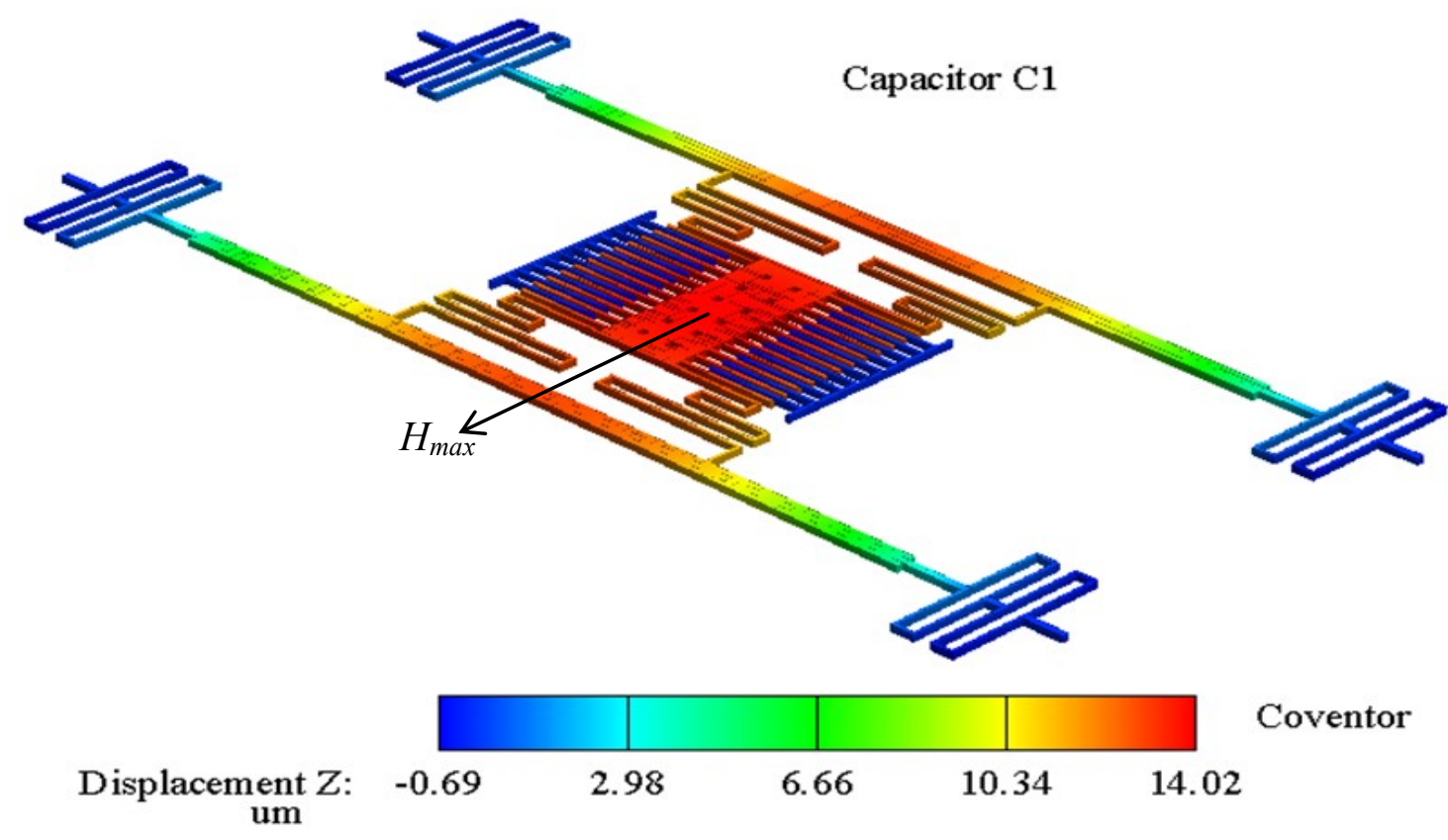

(a) 

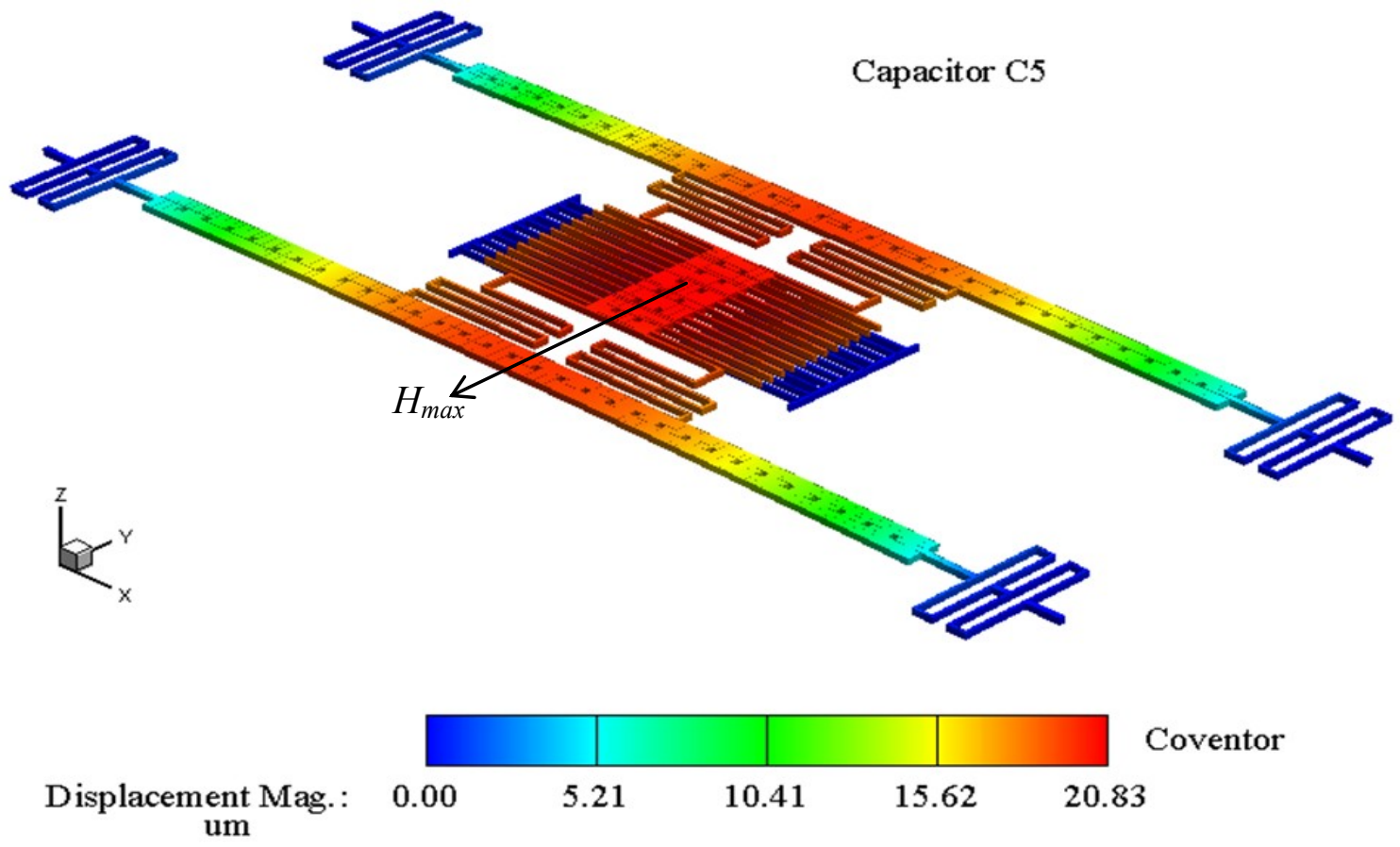

(b)
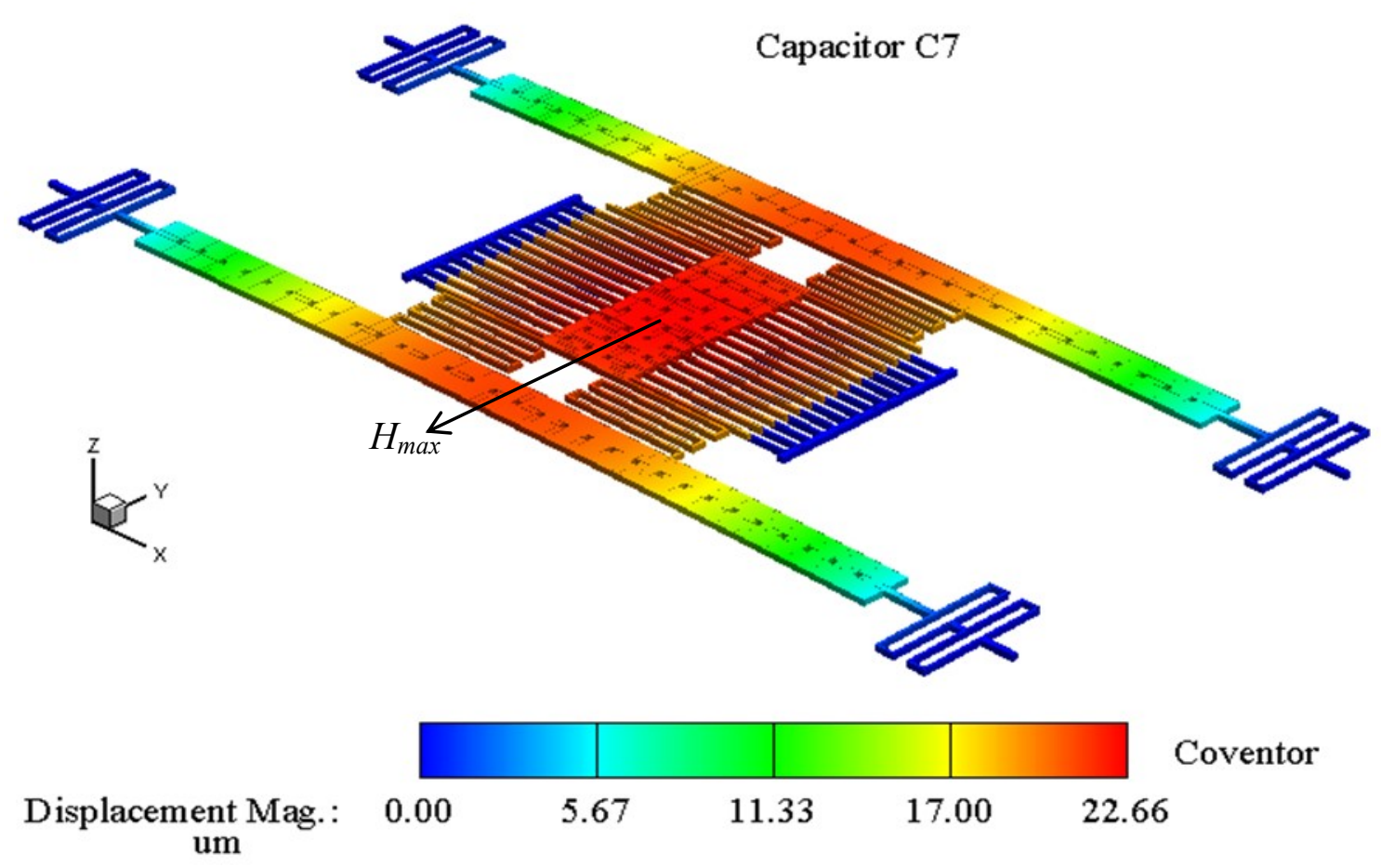

(c)

Figure 5-7 Deformations of capacitors (a) C, (b) C2 and (c) C7 


\subsection{Effect of geometric parameters on the maximum heights}

In this section, the effects of the variation of the geometric parameters such as length and width of the moving fingers and the fixed fingers of the capacitors are discussed based on the simulation results presented in section 5-4. The effect of the moving fingers and the moving plate on the maximum deflection, $H_{\max }$, of the curve up beams is discussed.

The simulations of capacitors $\mathrm{C} 1$ and $\mathrm{C} 3$ showed heights of $14.07 \mu \mathrm{m}$ and the $14.7 \mu \mathrm{m}$, respectively. These capacitors have identical geometric parameters except the length of the fingers and the gap between the fingers (Table 5-2). A $100 \mu \mathrm{m}$ change in length increased the vertical heights by $0.7 \mu \mathrm{m}$. The gap between the fingers has no effect on the vertical heights of the capacitors. However, large gaps decrease the capacitance between the moving fingers and the fixed fingers.

In the designs of capacitors $\mathrm{C} 2, \mathrm{C} 4$, and $\mathrm{C} 5$ the length of the curved beam is set to $2500 \mu \mathrm{m}$. The widths of the curve up beams for $\mathrm{C} 2, \mathrm{C} 4$ and $\mathrm{C} 5$ are $50 \mu \mathrm{m}, 100 \mu \mathrm{m}$ and $100 \mu \mathrm{m}$, respectively. Capacitor $\mathrm{C} 4$ has $400 \mu \mathrm{m}$ long moving fingers whereas $\mathrm{C} 2$ and $\mathrm{C} 5$ have $350 \mu \mathrm{m}$ long fingers. This difference increased the vertical heights by $1.96 \mu \mathrm{m}$ between $\mathrm{C} 4$ and $\mathrm{C} 2$. Similarly the vertical height difference between $\mathrm{C} 4$ and $\mathrm{C} 5$ is $0.91 \mu \mathrm{m}$ which resulted from different finger lengths. As the width of the curve-up beam further increased to $150 \mu \mathrm{m}$ the vertical heights increased as in the case for $\mathrm{C} 6$ and $\mathrm{C} 7$. The anchoring spring for $\mathrm{C} 7$ is slightly different from the rest of design in which the upper box spring is split into two. As a result, the stiffness its anchoring spring is increased and the heights $H_{\max }$ is reduced. The difference between the deflections of C6 and C7 is $0.33 \mu \mathrm{m}$. Capacitor C8 is similar to the design of capacitor C2. The capacitor C9 has curve-up beam of $100 \mu \mathrm{m}$ longer compared to $\mathrm{C} 10$ and $0.21 \mu \mathrm{m}$ change in the heights is obtained.

The dimensions of the moving plate, is another factor that influence the vertical height difference between the fixed fingers and the moving fingers. This is indirectly dependent on the number of moving fingers on the moving plate. The moving plates of capacitors $\mathrm{C} 3, \mathrm{C} 4, \mathrm{C} 6, \mathrm{C} 7$ and $\mathrm{C} 10$ have equal length. The widths of the moving plates of the capacitors C3, C4 and C10 are $200 \mu \mathrm{m}$ while the widths of the $\mathrm{C} 6$ and $\mathrm{C} 7$ are $300 \mu \mathrm{m}$. The increase in width of the moving plate from $200 \mu \mathrm{m}$ to $300 \mu \mathrm{m}$ only caused a $0.02 \mu \mathrm{m}$ on the height difference between the moving and the fixed fingers. It is possible to conclude that the change in the width of the plate does have little 
influence on the deformation of the moving fingers and the curve-up beams. Therefore, the addition of the moving plates, moving fingers and the plate supporting springs increase the maximum deflection, $H_{\max }$.

\subsection{Prototypes measurement and verification}

Capacitor s were fabricated using MetalMUMPs process run MM1201. The test set up presented in Section 3.6.1 is used for measuring and testing these capacitors. The experimental tests: novoltage and measurements with voltage are presented.

\subsubsection{No-voltage measurements}

The purpose of no-voltage measurements are helpful to verify the design principles whether the moving fingers are above the fixed fingers are vertically. The loose die image of the capacitors is shown in Figure 5-8. Measurements on the curve-up beams, fingers, moving plate, anchoring springs and serpentine springs are all illustrated. The measured deflections, $H_{\max }$, between the moving fingers and the fixed fingers are listed in Table 5-4 for randomly selected five loose dies. The SEM image of capacitor C7 is illustrated in Figure 5-9 (a) with its parts labeled. The vertical height difference, $H_{\max }$, caused by the residual stress gradient is marked in Figure 5-9 (b). The trench surface is taken as a reference plane for all measurements.

The surface maps of the capacitors are inspected using microscope and by measuring the surface maps on the profile analyzer. The profile plots on selected areas of the maps are illustrated in Figure 5-10 to Figure 5-12 for C1, C5 and C7, respectively. Profile plots of C2-C4, C6, and C8$\mathrm{C} 10$ are included in Appendix C. 


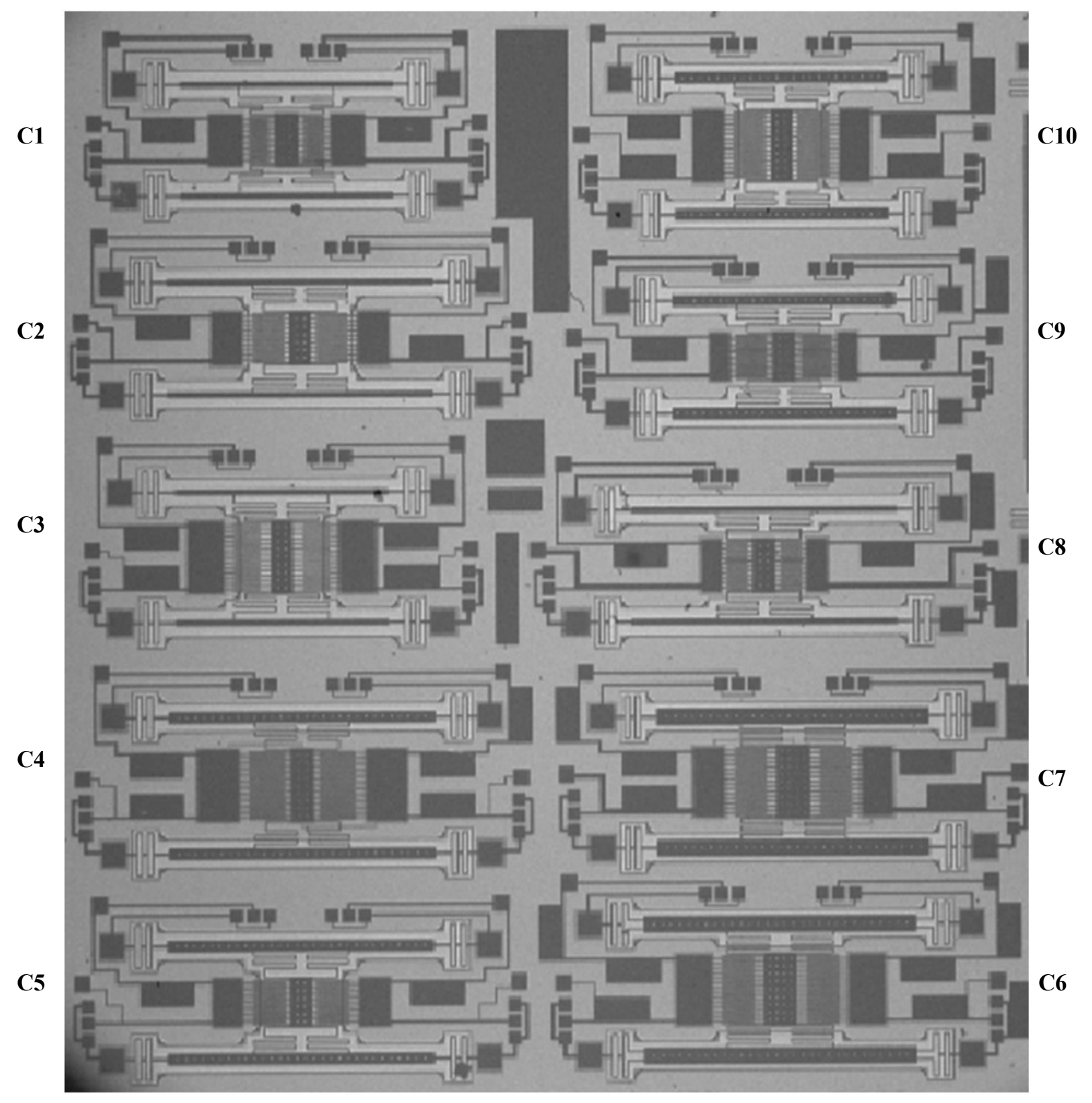

Figure 5-8 Loose die image C1-C10 


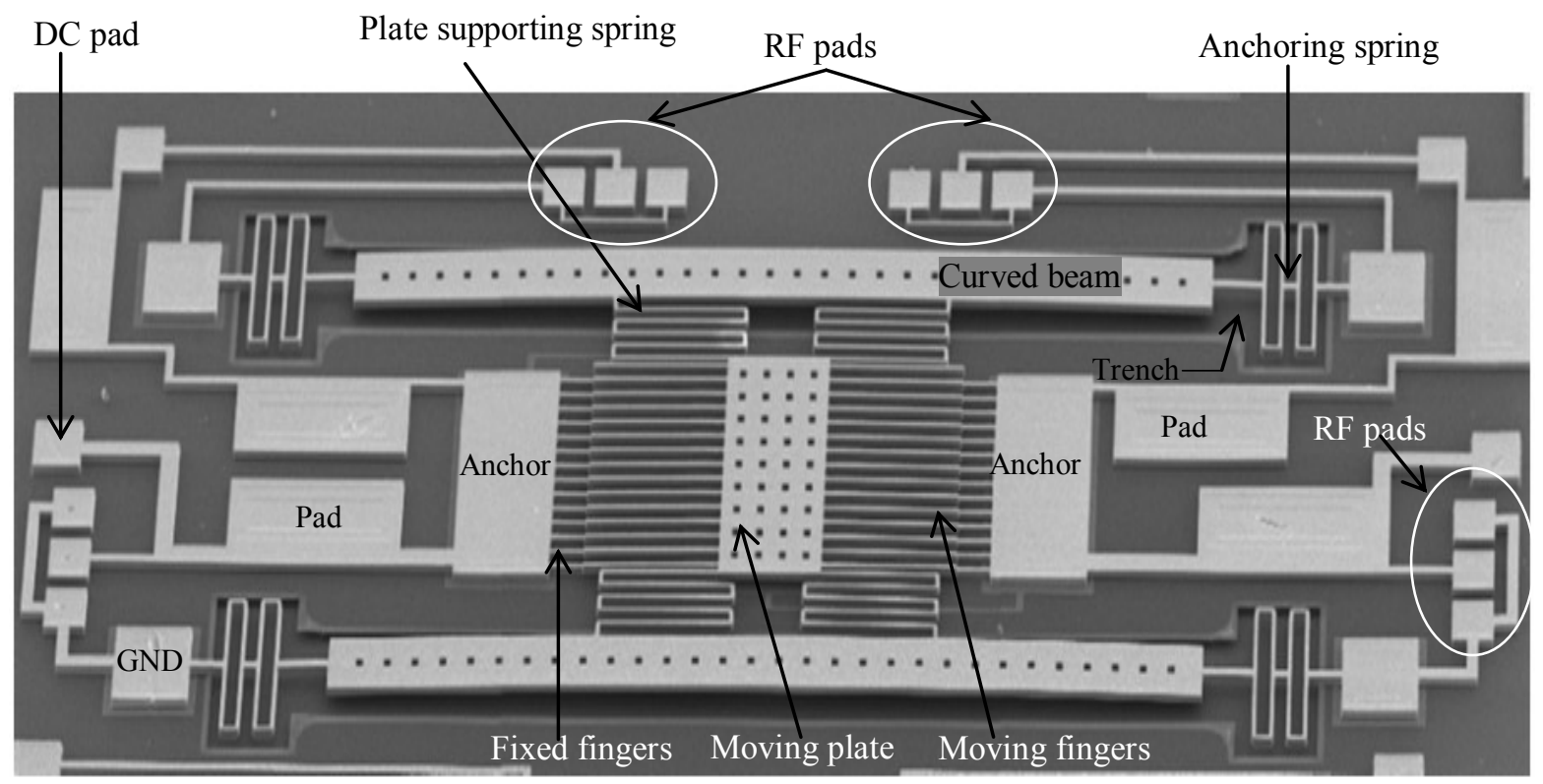

(a)

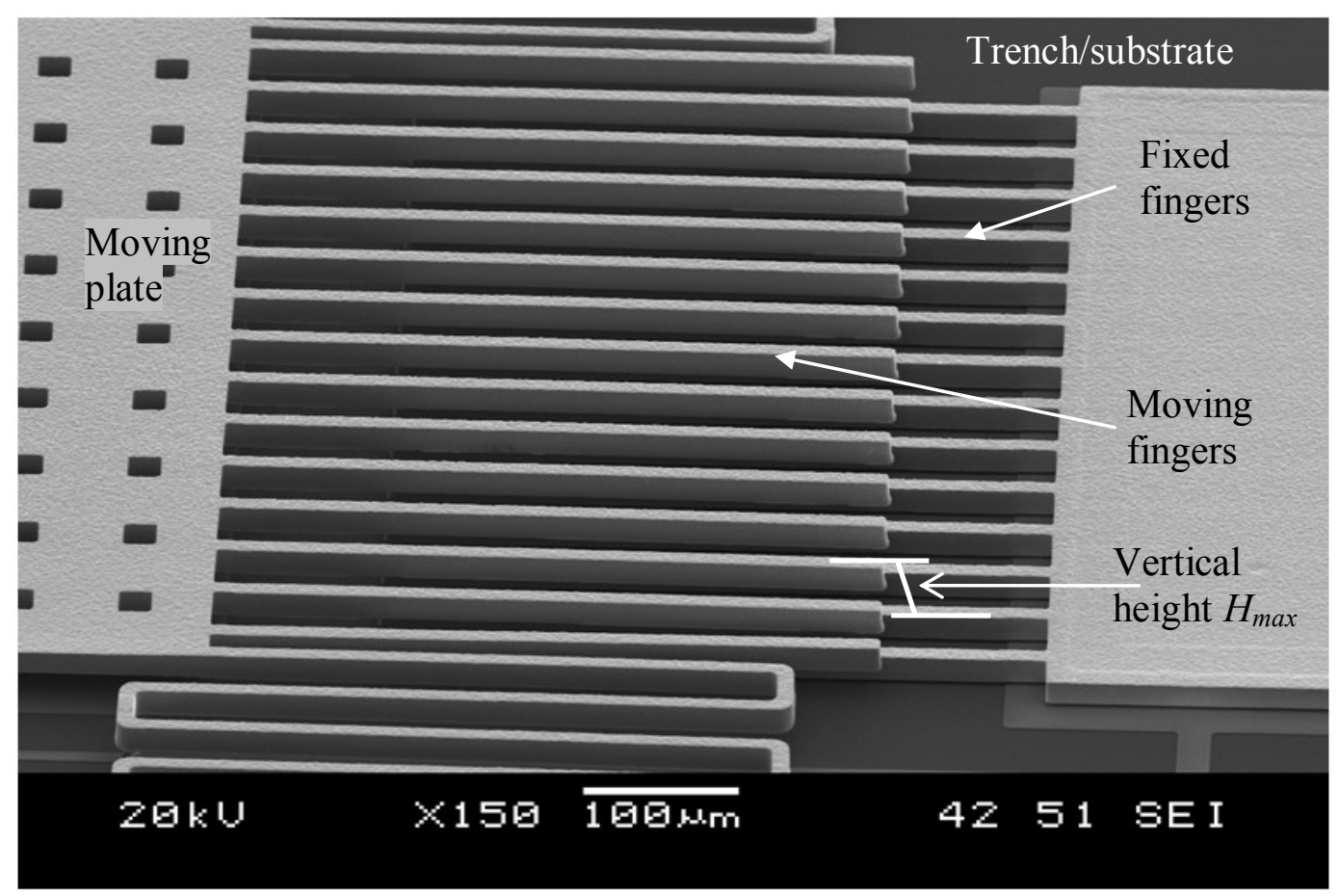

(b)

Figure 5-9 SEM micrograph of tunable capacitor C7 


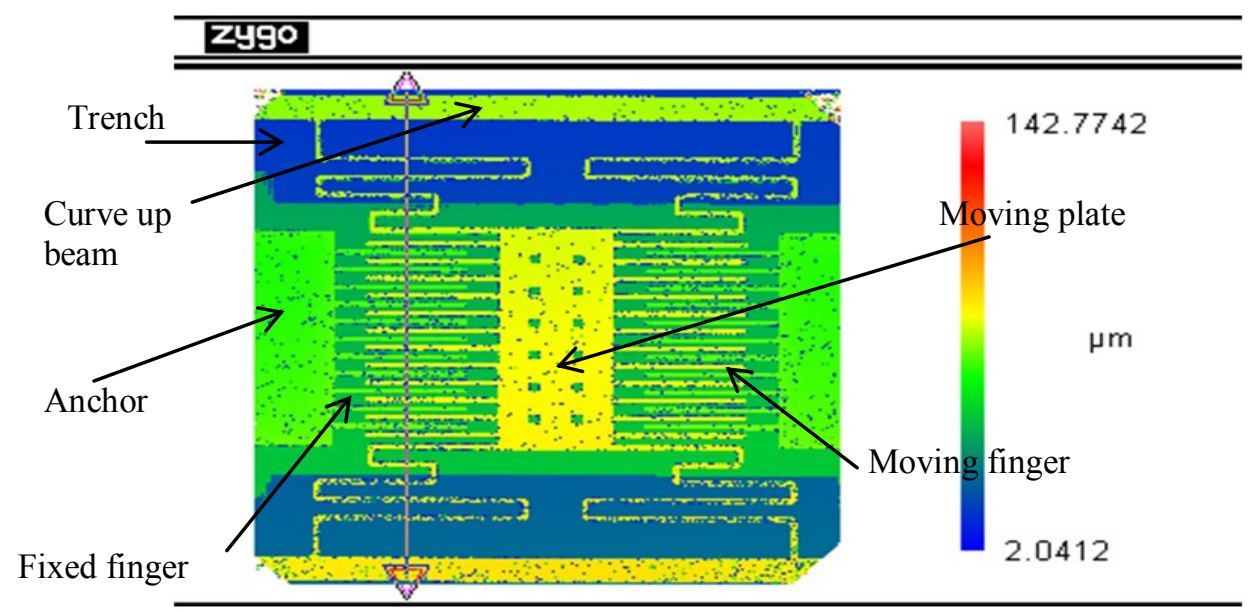

(a)

贫 Zygo $\quad$ Profile Plot
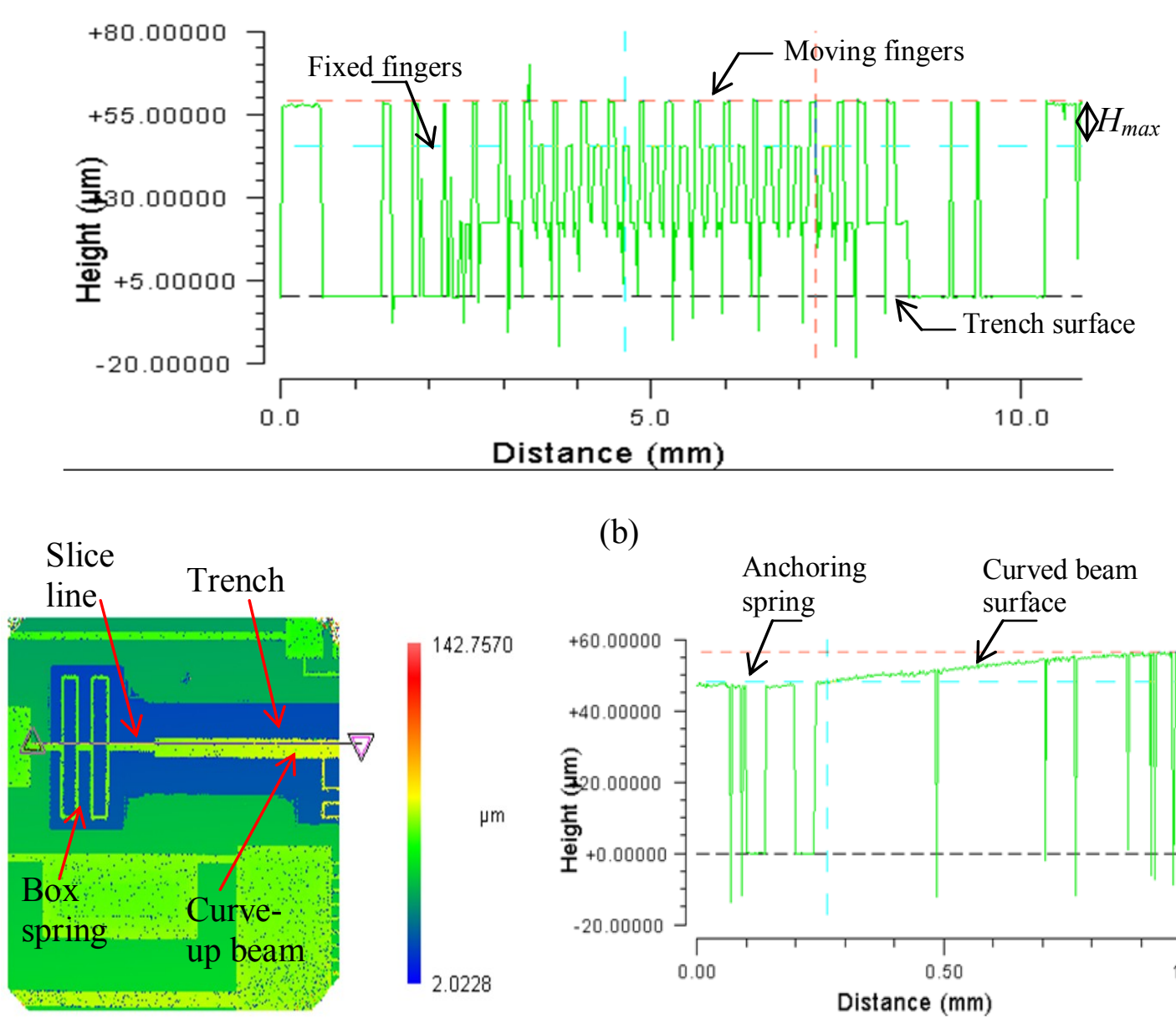

(b)

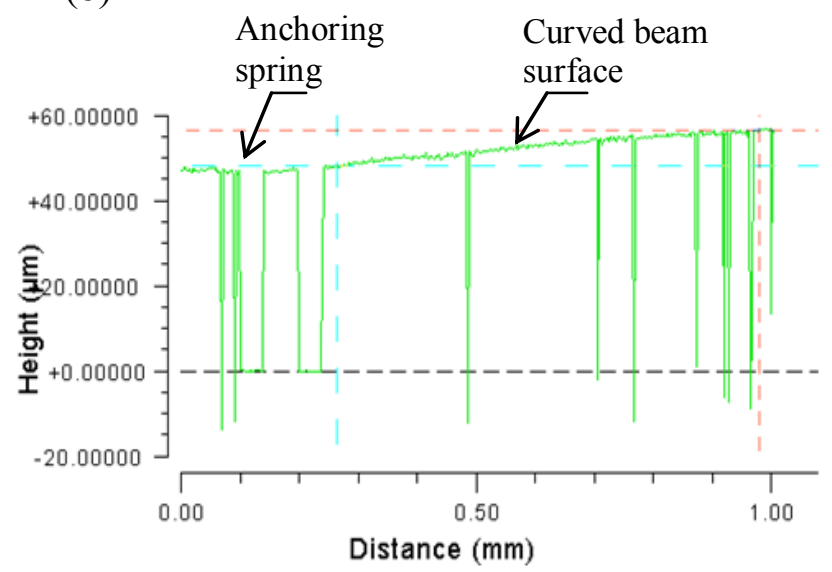

(c)

Figure 5-10 Capacitor C1 (a) surface map and (b) profile plot (c) curve-up beam plot 


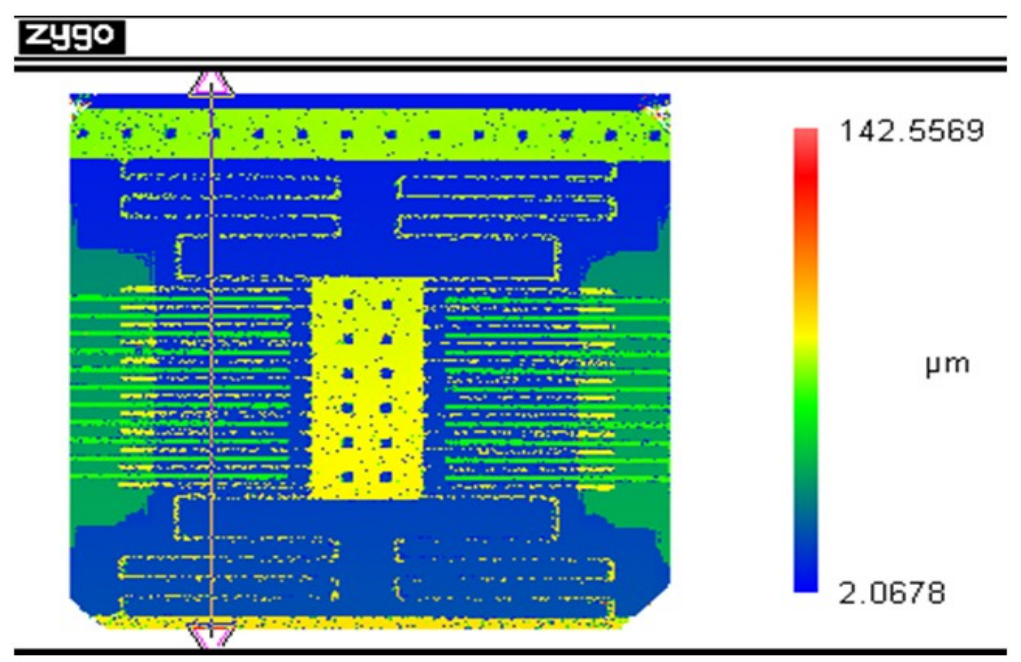

(a)

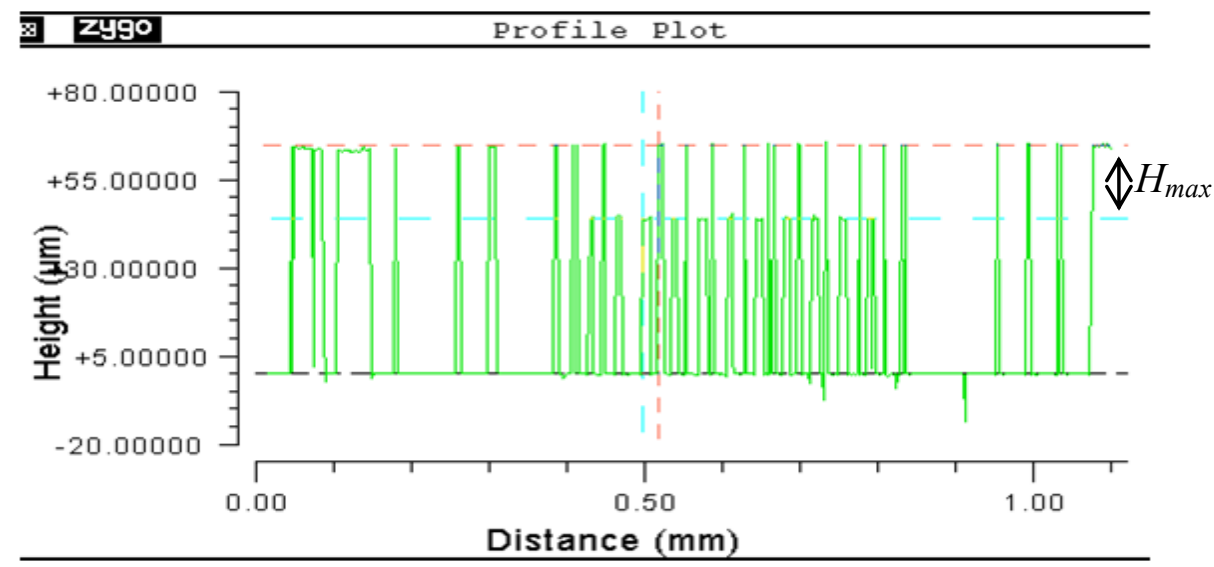

(b)
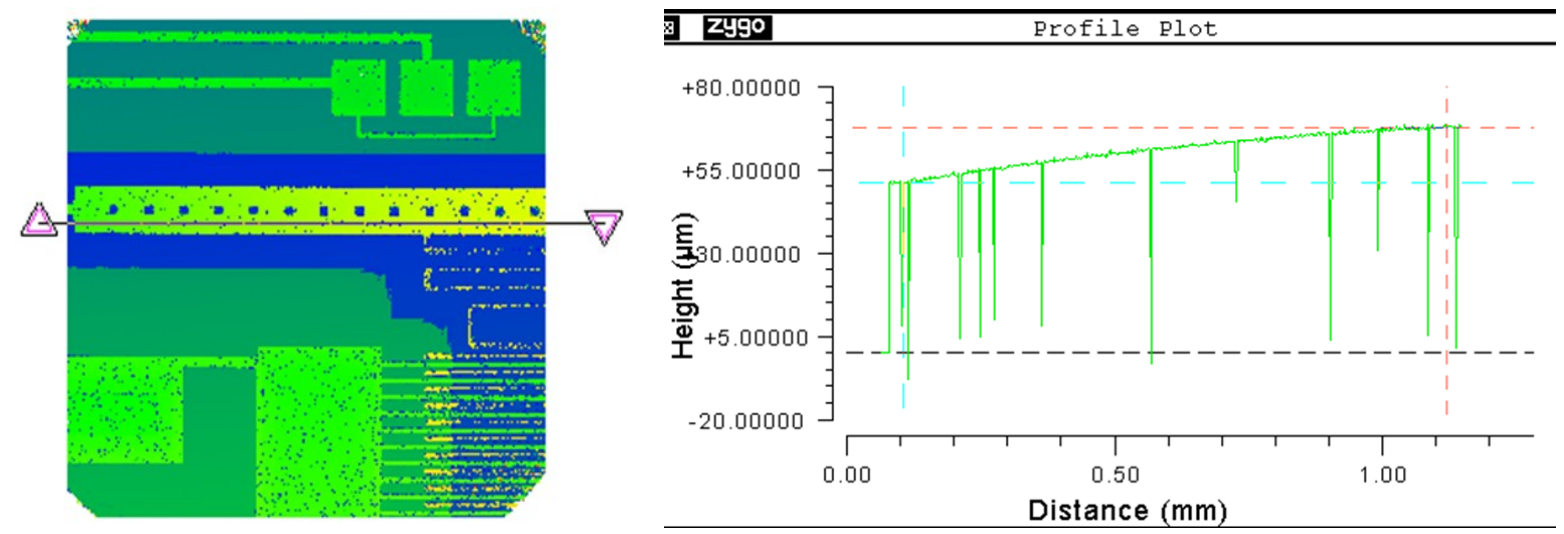

(c)

Figure 5-11 Capacitor C5 (a) surface map and (b) profile plot (c) curve-up beam plot 


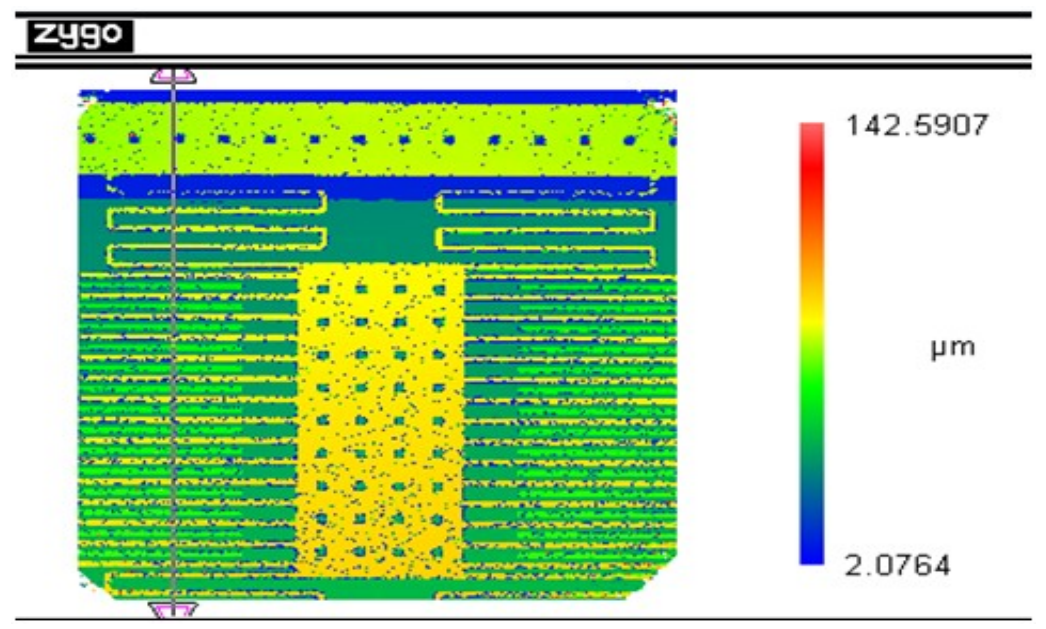

(a)

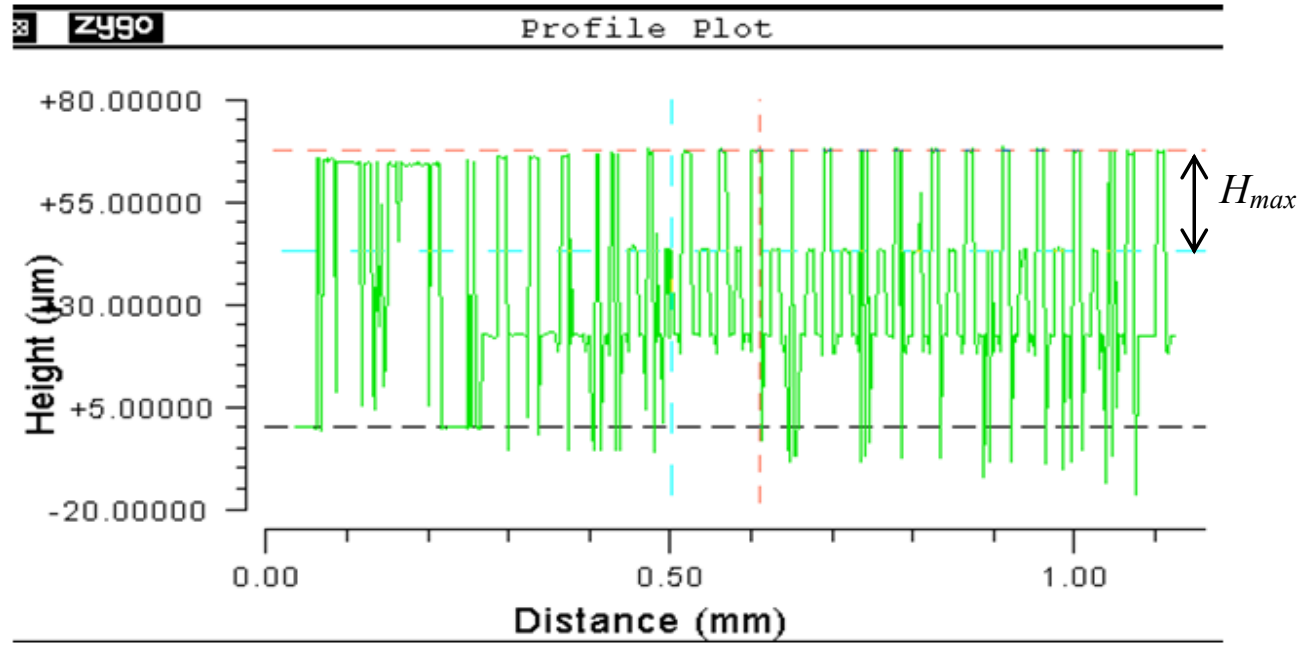

(b)
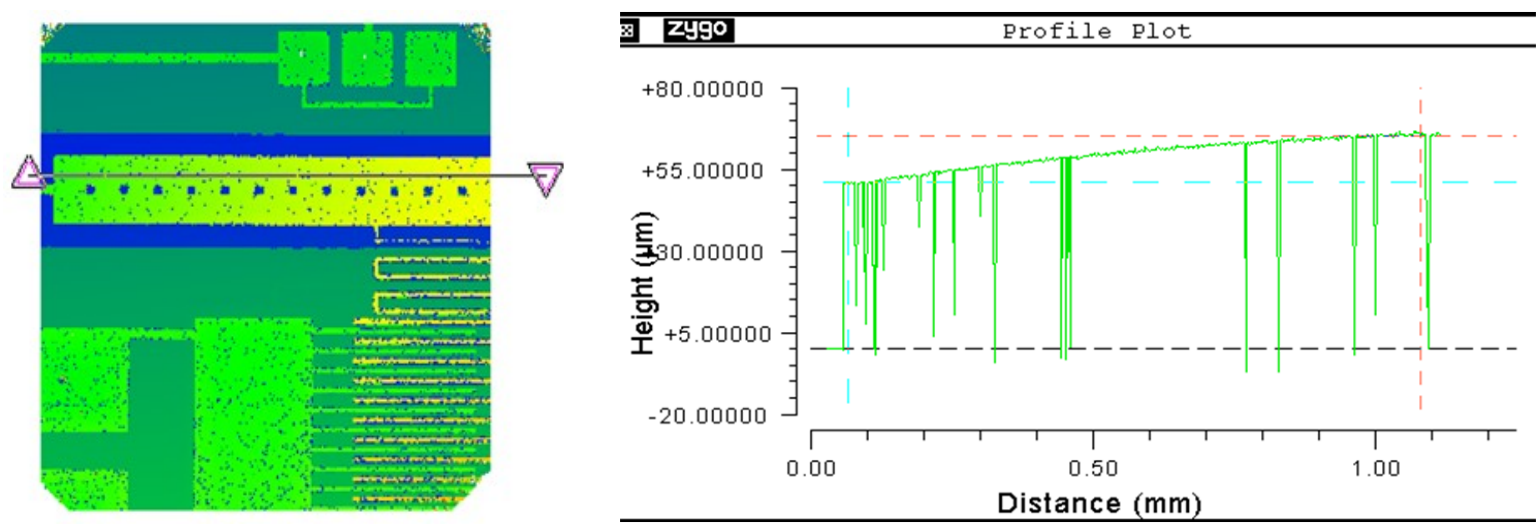

(c)

Figure 5-12 Capacitor C7 (a) surface map and (b) profile plot (c) curve-up beam plot 
Table 5-4 Measured maximum height $\left(H_{\max }\right)$

\begin{tabular}{ccccccccccc}
\hline Capacitor & C1 & C2 & C3 & C4 & C5 & C6 & C7 & C8 & C9 & C10 \\
\hline Die1 & 12.8 & NA & 12.76 & 18.88 & 17.56 & 19.8 & 21.2 & 16.83 & 13.82 & 13.21 \\
Die2 & NA & 17.1 & 13.3 & 21.65 & 20.17 & NA & 23.64 & 18.51 & 16.78 & 15.71 \\
Die3 & 12.7 & 13.76 & 13.24 & 20.03 & 19.79 & 23.62 & 25.06 & 15.62 & 18.80 & 15.1 \\
Die4 & 13.7 & 18.9 & 14.15 & 21.73 & 20.64 & 23.74 & 24.38 & 19.02 & 17.05 & 18.04 \\
Die5 & 13.44 & 18.69 & 14.67 & 21.69 & 21.00 & 23.14 & 22.82 & 19.35 & NA & 15.91 \\
\hline
\end{tabular}

$\mathrm{NA}=$ failed components

\subsubsection{Simulated Vs. measured height}

Comparison among the simulated and the measured $H_{\max }$ values show that the deflections after fabrication are different from the simulated values. The first possible factor could be the stress gradients experienced by the beams may differ in magnitude [101]. The deflections of the beams highly depend on the bending moment in the metal films [112]. The second factor for the discrepancy between the difference in the measured and the simulated results is the variation in the thickness of the metal films [113] during deposition. The thickness of the metal ranges from $17 \mu \mathrm{m}-23 \mu \mathrm{m}$ [103]. However, the nominal thickness used for the simulated $H_{\max }$ values is $20.5 \mu \mathrm{m}$ including the coating gold. The randomness of the thickness has significant effect on the stress gradient parameter so that it randomly changes the measured $H_{\max }$. Because of such variable thickness of the deposited metal over the chip area, the value of the measured $H_{\max }$ could differ from simulated values. Higher residual stress gradients on thinner films cause large deflection compared to thicker films. Figure 5-13 shows graphical comparison of simulated heights and measured heights. 


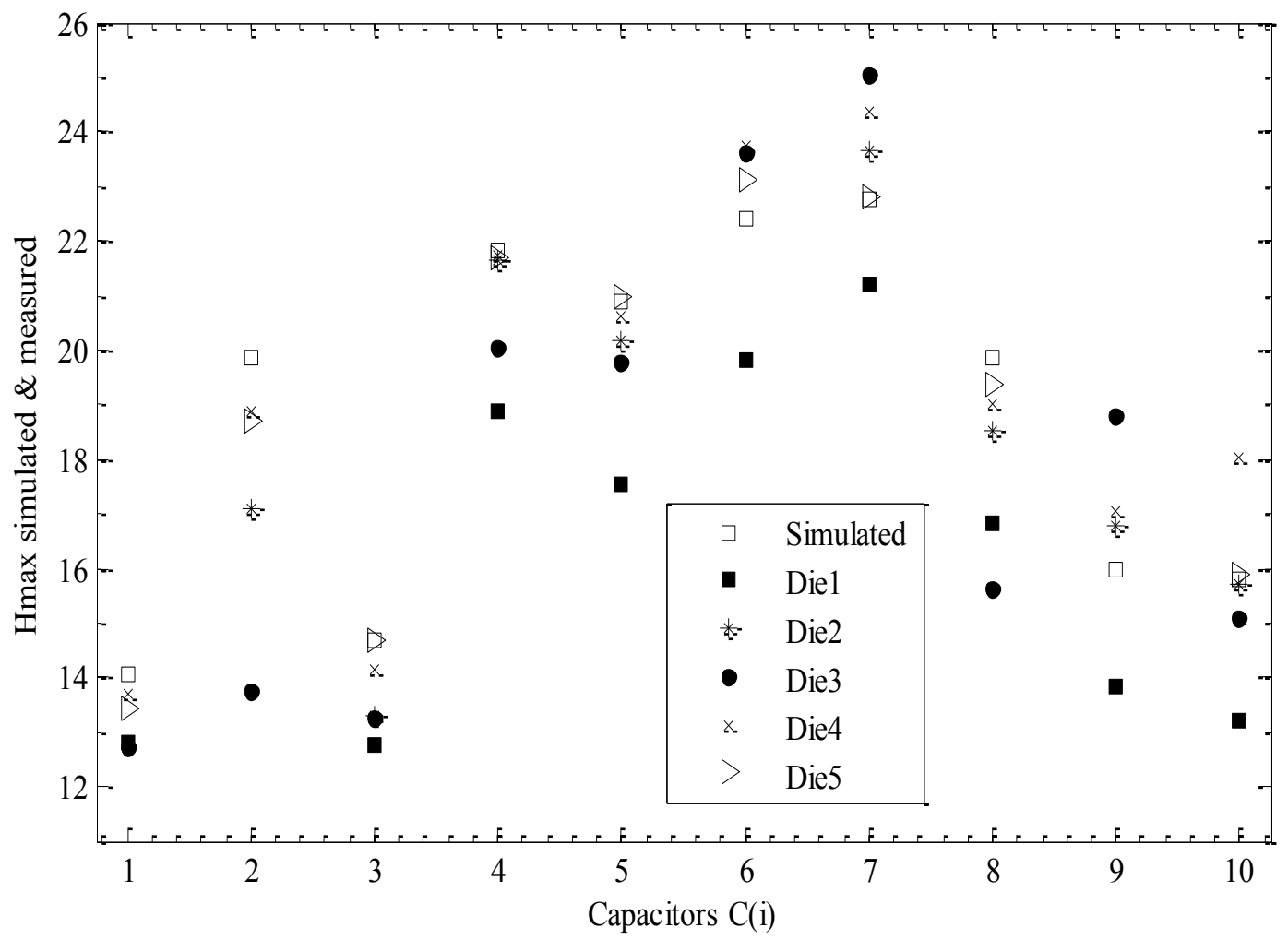

Figure 5-13 Simulated and measured $H_{\max }$ values

\subsubsection{Electromechanical measurements-voltage test}

In this section, the displacement travelled by the moving fingers of the capacitors is measured in the presence of DC voltages. These voltage tests are performed on capacitors of Die \#4. A similar procedure described in Section 4.7.2 is followed to measure the downward displacement of the moving plates and the moving fingers of the capacitors. The surface of the trench is used as a reference to measure the changes in the displacements. The value of the displacements measured at each actuation voltage is compared with no-voltage measurements. The distance travelled by the moving fingers of the capacitor is obtained by subtracting the displacements measured at any actuation voltage from the no-voltage measurements. The measured displacements for actuation voltages from 0V-200V are shown in Table 5-5. The broken lines in the table show the failure of the capacitors at that voltage because of the short-circuiting of the moving and the fixed fingers. 
Figure 5-14 and Figure 5-15 show the surface maps and profile plots of capacitor $\mathrm{C} 4$ and $\mathrm{C} 7$ respectively. The measured heights of the moving plates above the trench are shown in the graphs.

Table 5-5 Electromechanical measurements-change in displacements

\begin{tabular}{|c|c|c|c|c|c|c|c|c|c|}
\hline C(i) & OV & $25 \mathrm{~V}$ & $50 \mathrm{~V}$ & $75 \mathrm{~V}$ & $100 \mathrm{~V}$ & $125 \mathrm{~V}$ & $150 \mathrm{~V}$ & $175 \mathrm{~V}$ & $200 \mathrm{~V}$ \\
\hline $\mathrm{C} 1$ & 13.7 & 0.0424 & 0.5061 & 0.9067 & 1.6579 & 2.1458 & 4.4715 & $\begin{array}{l}----@ \\
162.5 \mathrm{~V}\end{array}$ & \\
\hline $\mathrm{C} 2$ & 18.9 & 0.2422 & 1.036 & 1.1974 & 1.6612 & 2.58 & 3.2156 & 5.0052 & ----- \\
\hline $\mathrm{C} 3$ & 14.15 & 0.4886 & 0.7217 & 0.837 & 1.5619 & 1.9875 & 2.8981 & 3.3794 & $\begin{array}{c}3.9894-- \\
-\end{array}$ \\
\hline $\mathrm{C} 4$ & 21.73 & 0.4961 & 0.9156 & 2.1838 & 3.9931 & ------- & & & \\
\hline $\mathrm{C} 5$ & 20.64 & 0.0675 & 0.8531 & 1.4034 & 2.0217 & ----- & & & \\
\hline C6 & 23.74 & 0.2840 & 0.7205 & 1.1415 & 1.6089 & 3.0038 & 4.8086 & ------- & \\
\hline $\mathrm{C} 7$ & 24.38 & 0.1035 & 0.8763 & 1.6667 & 2.1396 & 3.6564 & 6.4825 & $\begin{array}{c}7.7423 \\
@ 162.5 \mathrm{~V} \\
\end{array}$ & ----- @ \\
\hline C8 & 19.02 & 0.2055 & 0.7172 & 1.3508 & 2.0392 & 3.3947 & 4.0301 & 5.6189 & $\begin{array}{c}6.9995 \\
@ 187.5 \mathrm{~V}\end{array}$ \\
\hline C9 & 17.05 & 0.5322 & 0.863 & 1.6525 & 2.6782 & $\begin{array}{c}2.9252 \\
@ 112.5 \mathrm{~V} \\
\end{array}$ & @ $125 \mathrm{~V}$ & & \\
\hline $\mathrm{C} 10$ & 18.04 & 0.4389 & 0.7169 & 1.024 & 1.9527 & 2.4546 & 3.4054 & -------- & \\
\hline
\end{tabular}



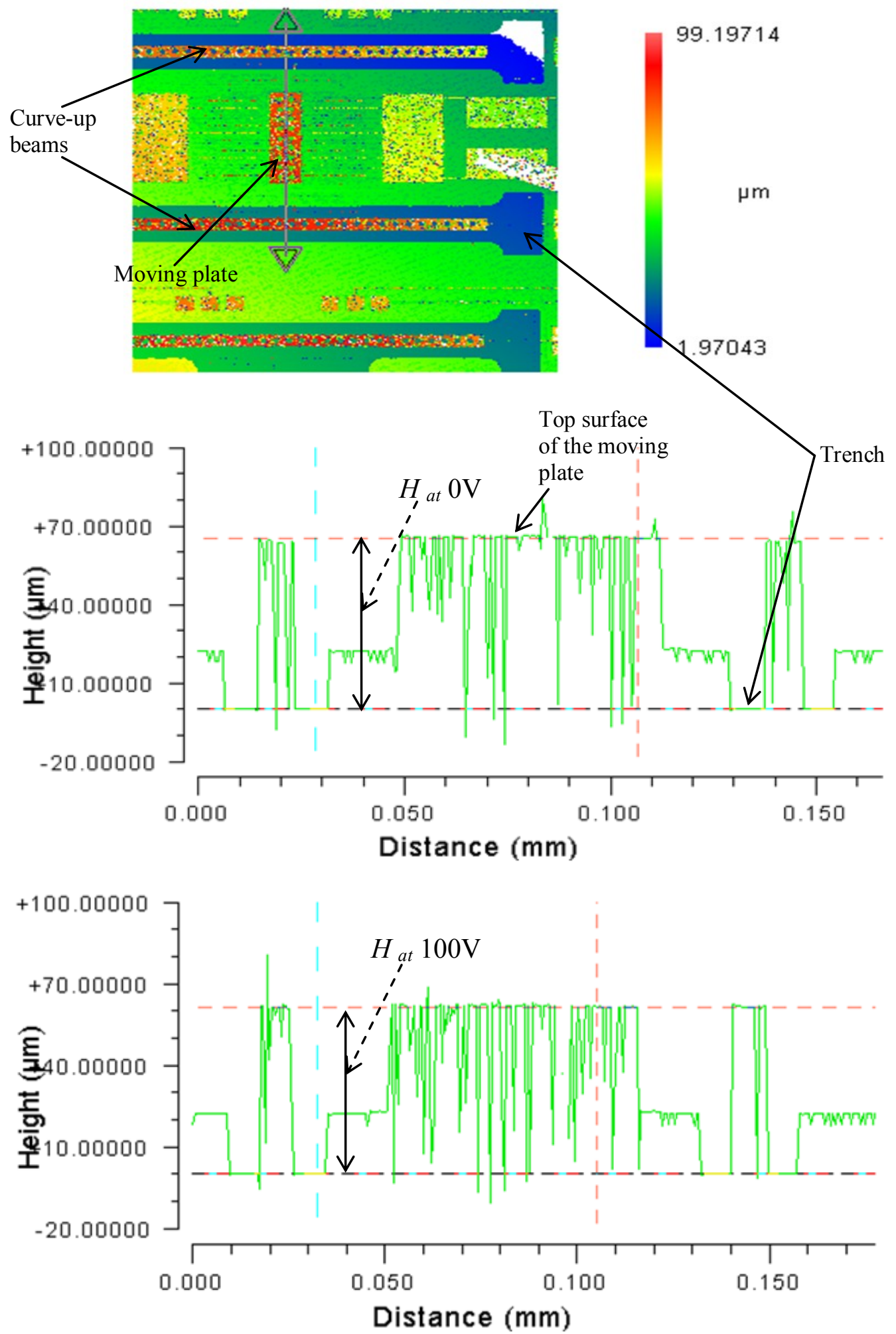

Figure 5-14 Measured profile plot of C4 at $0 \mathrm{~V}$ and $100 \mathrm{~V}$ 
From the profile plots depicted in Figure 5-14, the height differences are compared for actuation voltages of $0 \mathrm{~V}$ and $100 \mathrm{~V}$. At $0 \mathrm{~V}$ the moving plate is at a height of $H_{\max }=65.34 \mu \mathrm{m}$ above the trench. When the voltage is increased to $100 \mathrm{~V}$ the height $H_{\max }=61.43 \mu \mathrm{m}$. The height difference resulting from $0 \mathrm{~V}$ to $100 \mathrm{~V}$ is $3.91 \mu \mathrm{m}$ which shows that the moving plate and the moving fingers moved approximately $4 \mu \mathrm{m}$ at $100 \mathrm{~V}$. In Figure 5-15 the measured displacements of capacitor C7 are illustrated for $0 \mathrm{~V}, 100 \mathrm{~V}, 150 \mathrm{~V}$ and $162.5 \mathrm{~V}$.
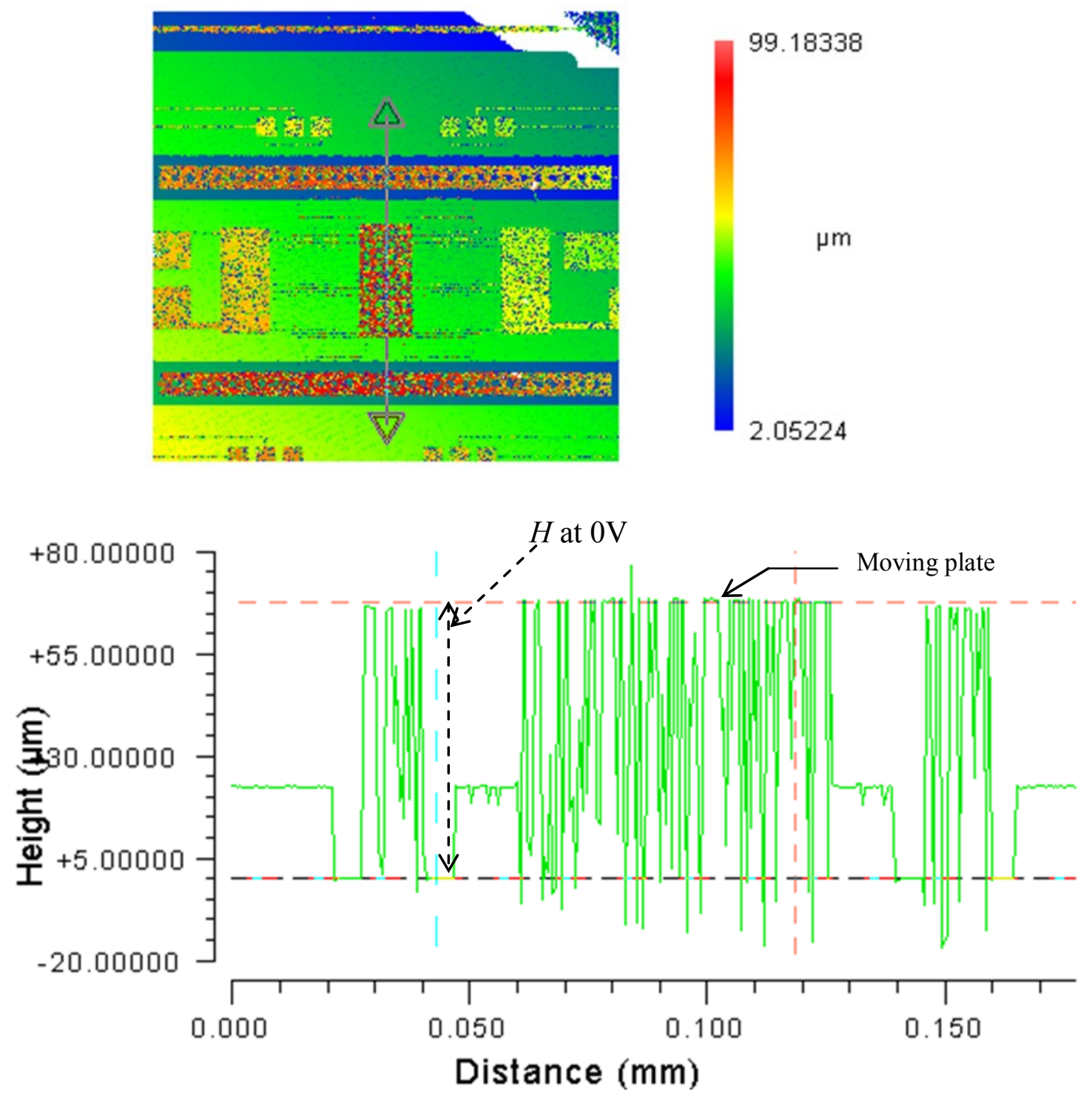

(a) 


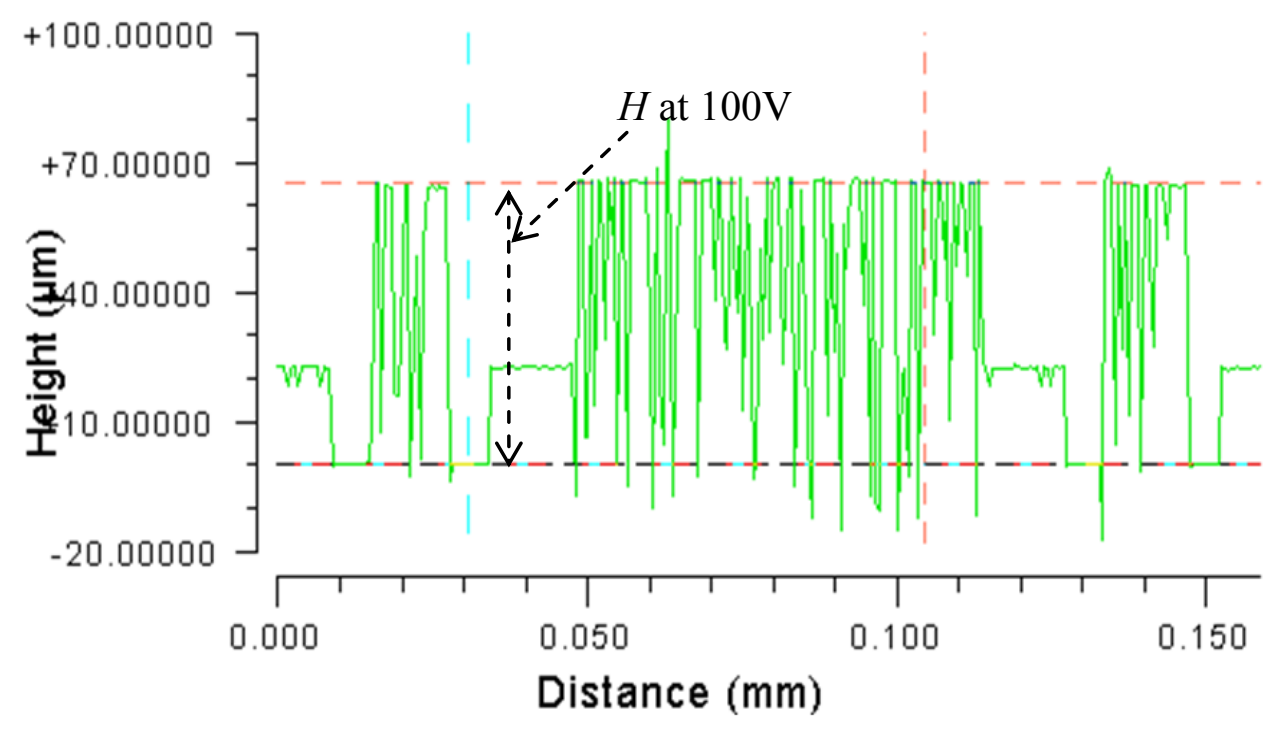

(b)

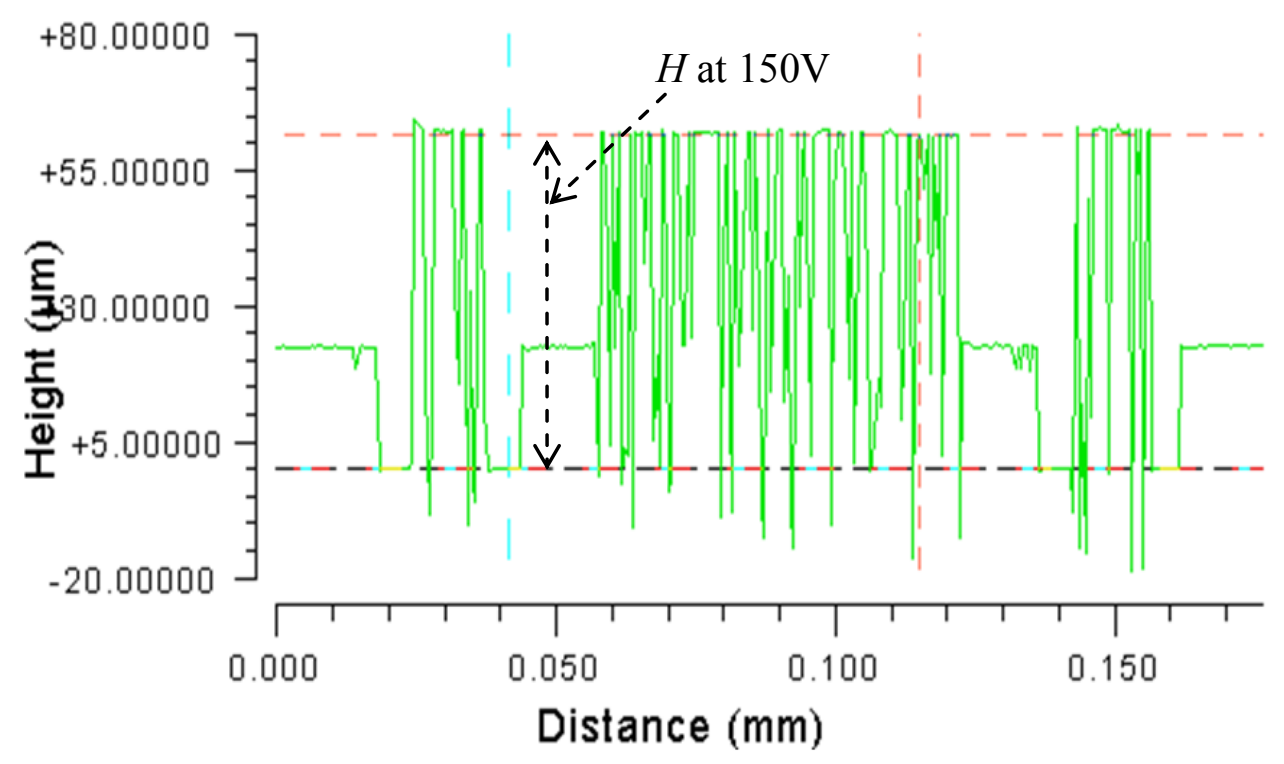

(c) 


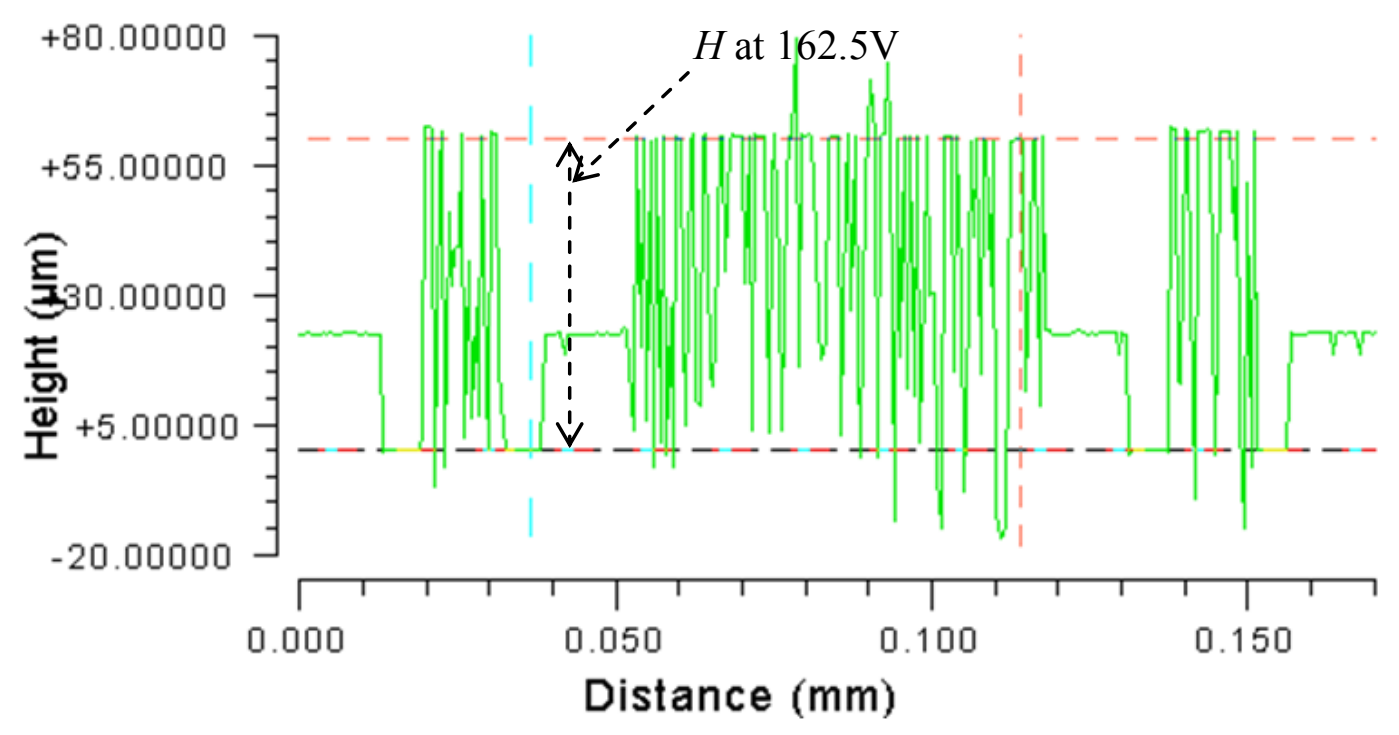

(d)

Figure 5-15 Measures profiles of C7 (a) 0V, (b) $100 \mathrm{~V}$, (c) $150 \mathrm{~V}$ and (d) $162.5 \mathrm{~V}$ From the profile measurements of Figure 5-15, at $0 \mathrm{~V}$ the moving plate is at a height of $67.69 \mu \mathrm{m}$ above the trench. When the voltage is increased to $100 \mathrm{~V}$ the height $H=65.21 \mu \mathrm{m}$ is measured. The height difference when the voltage is $2.48 \mu \mathrm{m}$ which shows that the moving plate and the moving fingers moved nearly $2.5 \mu \mathrm{m}$ at $100 \mathrm{~V}$. At $150 \mathrm{~V}$ and $162.5 \mathrm{~V}$, the measured heights are $H=61.26 \mu \mathrm{m}$ and $59.94 \mu \mathrm{m}$ respectively. Thus the corresponding measured displacement of moving plate at $150 \mathrm{~V}$ and $162.5 \mathrm{~V}$ are $6.43 \mu \mathrm{m}$ and $7.75 \mu \mathrm{m}$, respectively.

The DC voltage tests performed above showed that the moving plate and the moving fingers travelled down towards the fixed fingers. As the moving fingers move down, the capacitance between the moving fingers and the fixed fingers increase. Therefore, capacitance change is obtained by driving the actuators of the capacitors. In the following section the radio frequency performance test are presented to extract the capacitance via scattering (S) parameter measurements. The S-parameters are mainly used to extract the substrate capacitances and the quality factors of the tunable capacitors. 


\subsection{RF performance tests and analysis}

The measurements of scattering $(\mathrm{S})$ parameters of the tunable capacitors are presented here. The S-parameters are measured using a vector network analyzer over $0.8 \mathrm{GHz}$ to $65 \mathrm{GHz}$ frequency for actuation voltages of $0-100 \mathrm{~V}$ in $25 \mathrm{~V}$ step. The measured scattering parameters were used to extract the capacitance and calculate the quality factors of each capacitor. From the extracted capacitance substrate effects in terms of parasitic capacitance and power loss are investigated. The substrate effect on the tuning ratio of the capacitors is difficult to determine accurately with simulation or calculation at microwave frequencies since capacitance and resistances are dependent on the frequency of the RF signal.

\subsubsection{Scattering parameters}

Scattering parameters are elements that describe the behavior of circuits when a radio frequency signal is applied across the input ports of radio frequency circuits [111]. These parameters are usually measured at microwave and millimeter wave frequencies and are complex numbers with phase and magnitude. The S-parameters are normally measured with respect to the frequency and plotted in magnitude or $\mathrm{dB}$ form. For a two-port network the S-parameters are represented in matrix form as:

$$
[S]=\left[\begin{array}{ll}
S_{11} & S_{12} \\
S_{21} & S_{22}
\end{array}\right]
$$

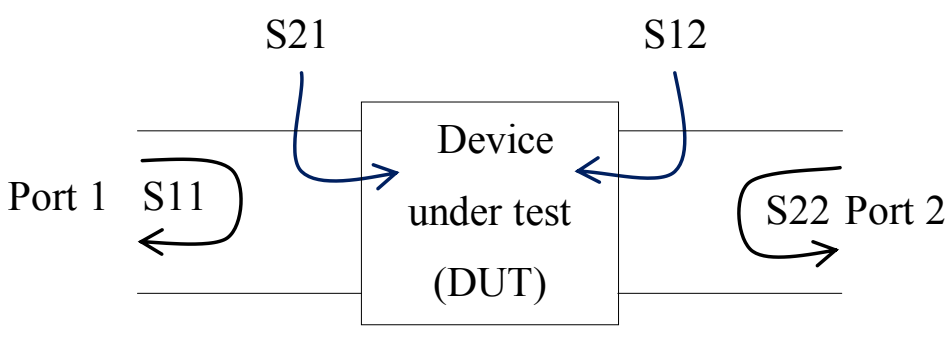

Figure 5-16 Two-port ntework

\subsubsection{S-parameter conversion equations}

The measured S-parameters were used to extract the capacitance and determine the quality factor of the capacitors. In order to extract the capacitance at the frequencies of the RF signal, the entire capacitor is modeled as a two port network of Figure 5-17. 


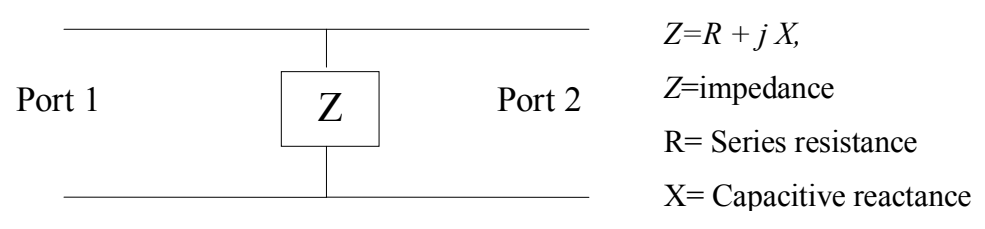

Figure 5-17 Microwave lumped model representation of capacitor

The S-parameters are converted to the equivalent ABCD parameters using the equation [111];

S-parameters $\quad \mathrm{ABCD}$ parameters in terms of S-parameters

$$
\left[\begin{array}{ll}
S_{11} & S_{12} \\
S_{21} & S_{22}
\end{array}\right]=\left[\begin{array}{lr}
\frac{\left(1+S_{11}\right)\left(1-S_{22}\right)+S_{12} S_{21}}{2 S_{21}} & Z_{0} \frac{\left(1+S_{11}\right)\left(1+S_{22}\right)-S_{12} S_{21}}{2 S_{21}} \\
\frac{1}{Z_{0}} \frac{\left(1-S_{11}\right)\left(1-S_{22}\right)-S_{12} S_{21}}{2 S_{21}} & \frac{\left(1-S_{11}\right)\left(1+S_{22}\right)+S_{12} S_{21}}{2 S_{21}}
\end{array}\right] \ldots \ldots(5
$$

After the conversion of the S-parameters to $\mathrm{ABCD}$ the tunable capacitor reduces to a single impedance network similar to Figure 5-17. $Z$ stands for the total impedance of the capacitors after conversion. From the impedance the imaginary and the real part are extracted. $R$ is the real part in the circuit (i.e. tunable capacitor) and $\mathrm{X}$ is the imaginary part that constitutes of the capacitance $\mathrm{C}$ of the capacitor and the parasitic capacitances from the substrate, pads and the strips. The quality factor, $\mathrm{Q}$, is given by

$$
Q=\frac{\text { imaginary part }}{\text { real part }}
$$

\subsubsection{S-parameter measurement setup}

The S-parameters were measured using a vector network analyzer (VNA) at University of Manitoba (UoM) in Advanced Radio Frequency Systems Laboratory (ARFSL). Figure 5-18 shows partial setup of the instruments used for S-parameter measurement. The equipments include a VNA with maximum output power of $-7 \mathrm{dBm}$ with a swing RF frequency of $300 \mathrm{MHz}-$ $65 \mathrm{GHz}$, DC voltage source, RF probes (on the probe station), camera and display screens. 


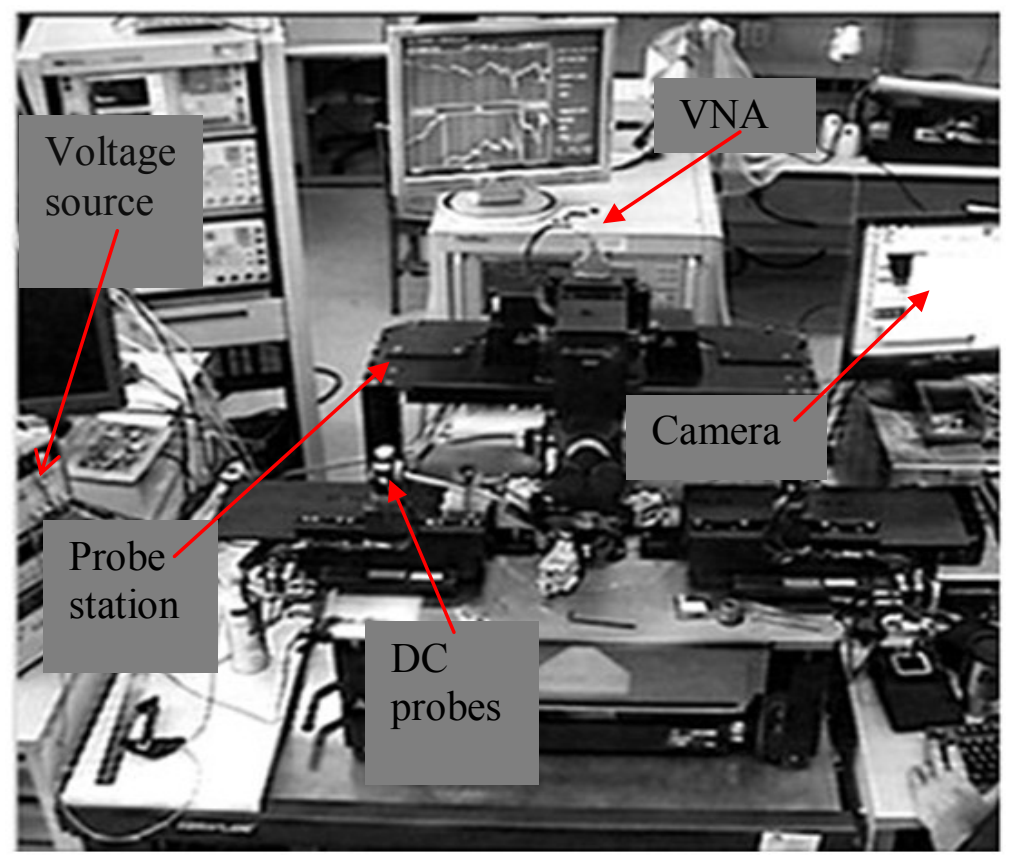

Figure 5-18 RF test setup at ARFSL (courtesy of CMC and UoM)

The S11 (dB) parameters that are measured at $0 \mathrm{~V}$ and 100Vare plotted in Figure 5-19 for capacitors $\mathrm{C} 1-\mathrm{C} 10$. The S11 parameters represent the reflection coefficient [111] of the RF signal at the input port. When the actuation voltages change from $0 \mathrm{~V}$ to $100 \mathrm{~V}$ the $\mathrm{S} 11$ parameters indicate the increase of the impedance. Based on the analysis of the S11 parameter graphs it is possible to conclude that (1) the capacitance change is very small over actuation voltages of $0 \mathrm{~V}$ to $100 \mathrm{~V}$, and (2) the distance travelled by the moving plates of the capacitor are small. In the electromechanical performance test higher displacement of the capacitors occur at higher voltages $>100 \mathrm{~V}$. S-parameter for higher voltages $>100 \mathrm{~V}$ were not measured since the biastee used cannot handle voltages above $100 \mathrm{~V}$. However, by extracting the capacitance from measured S-parameters over $0 \mathrm{~V}-100 \mathrm{~V}$ parasitic capacitances in the substrate, pads and metallic strips, quality factor and RF power losses were analyzed for design optimization. 

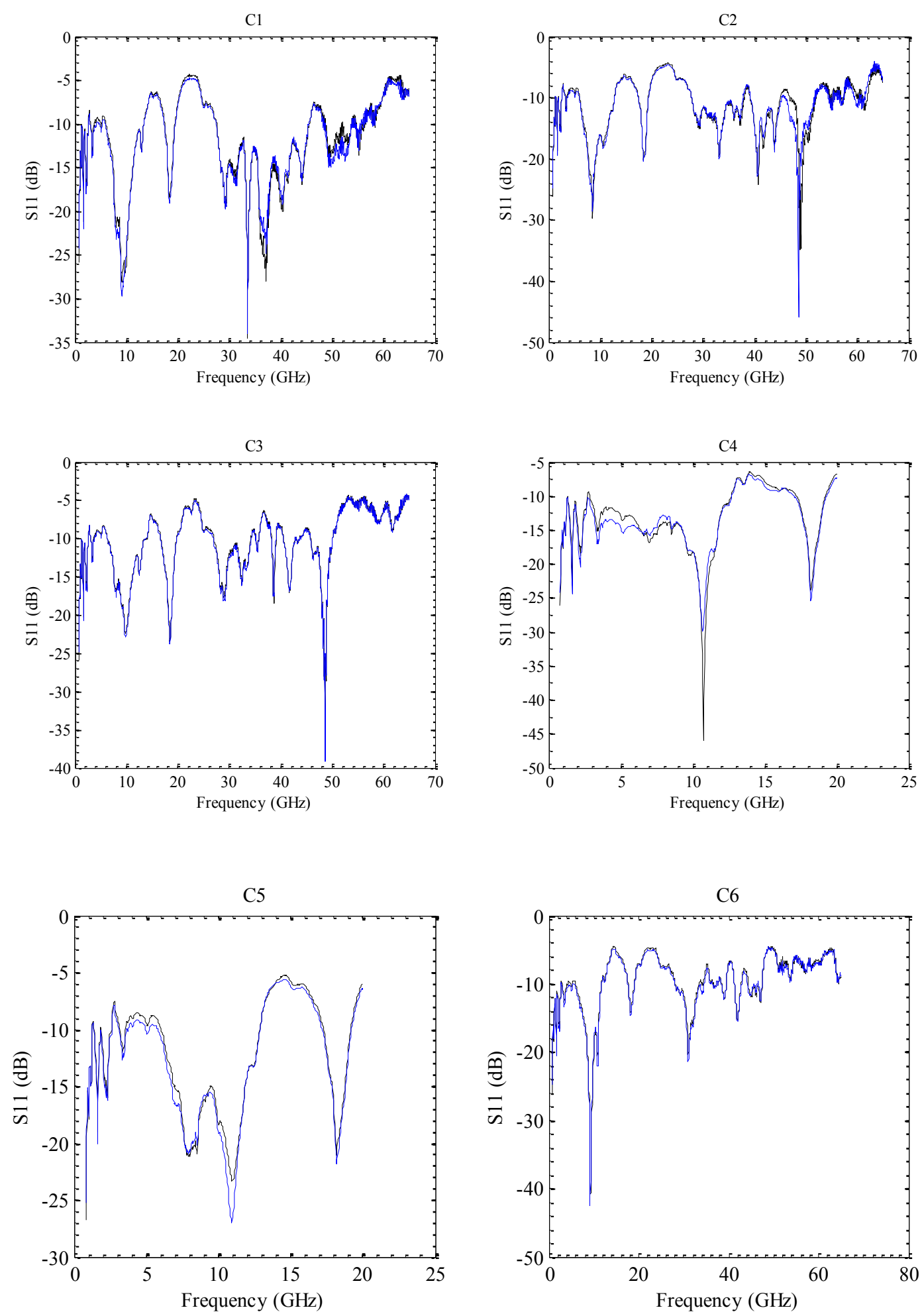

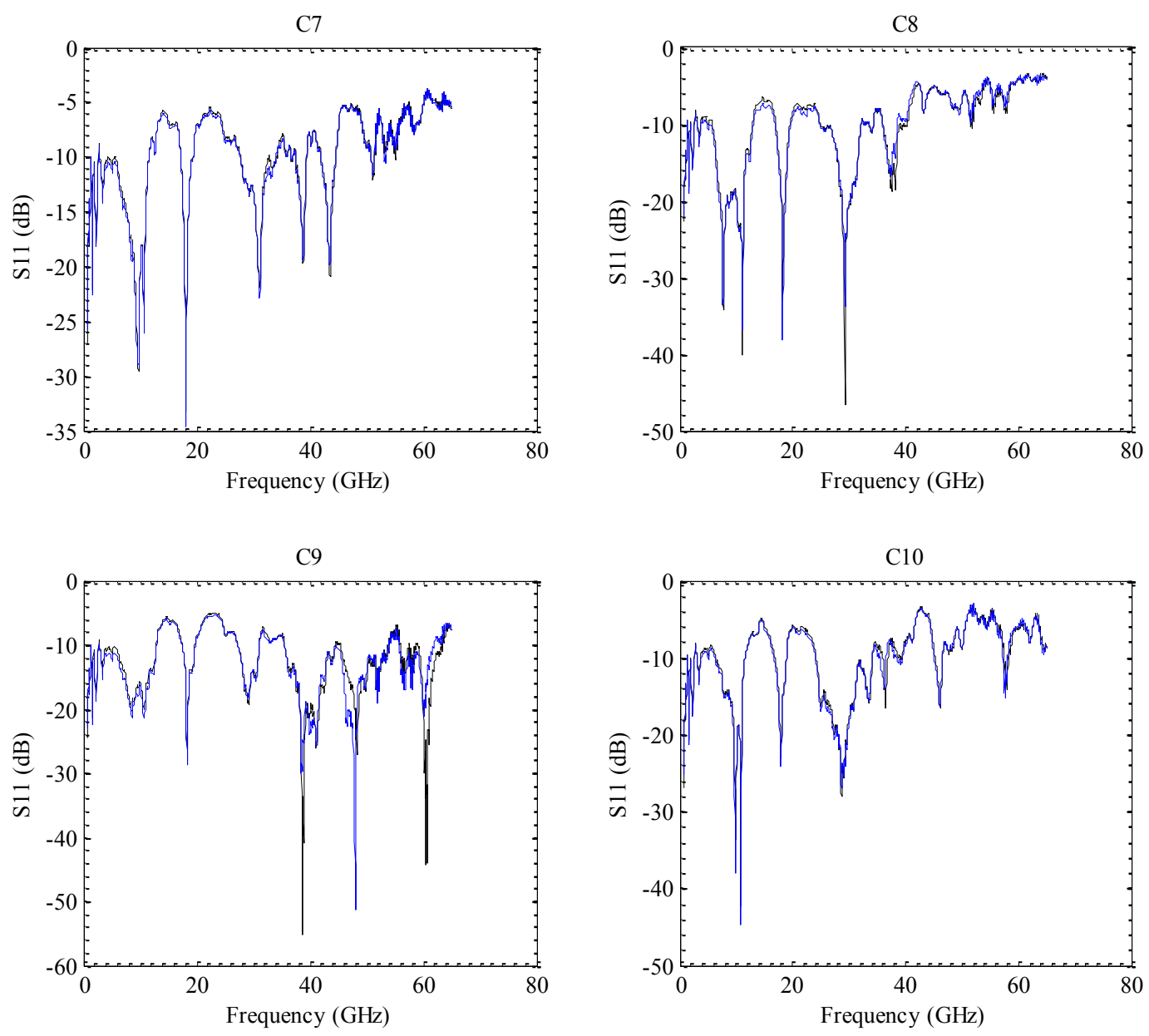

Figure 5-19 Measured S11 parameters of C1-C10 at $0 \mathrm{~V}$ and $100 \mathrm{~V}$

\subsubsection{Capacitance extraction and quality factor calculation}

The measured s-parameters of the capacitor were converted to equivalent transmission line parameters [111] which were modeled using lumped circuit equivalent to determine the Q factor and the tuning ratio over $0 \mathrm{~V}$ to $100 \mathrm{~V}$ voltage. This model considers the entire capacitor as a single element, $\mathrm{Z}$, with the RF signal input pads as the input and the output ports. Hence, the equivalent model includes the parasitic capacitance between the RF paths/strips, the RF pads, the MEMS anchors and the substrate. The limitation with this analysis is that the converted transmission line (sometimes known as ABCD parameters) consists of all the parasitic capacitances together with the tunable capacitance. 
The S-parameters were imported to a spreadsheet (.xls format) that is input to a Matlab program to be converted to $\mathrm{ABCD}$ parameters. From the impedance of the $\mathrm{ABCD}$ parameters, the capacitance and the quality factors were calculated.

The parasitic capacitance is high compared to the capacitance between the fingers. This is because the area possessed by the tunable capacitor is small in comparison with the support beam areas, DC pad areas, RF pad areas and RF strips. The RF and the DC pads produce high parasitic capacitance with the substrate which is because of the area and large side wall of the pads, strips and anchors. Since the metal has a thickness of $20.5 \mu \mathrm{m}$ it causes high lateral fringing capacitance. Another structure that causes high parasitic capacitance is the nitride dielectric layer which has dielectric constant of 8 . The silicon dioxide, sandwiched between the silicon substrate and the nitride layer, also causes high parasitic capacitance and RF power surge. Table 5-6 presents the extracted capacitance and the tuning ratios calculated.

The quality factors at $0.8 \mathrm{GHz}$ and $1.24 \mathrm{GHz}$ are shown in Table $5-7$ and 5-8 for each capacitor, respectively. The $\mathrm{Q}$ factor plots of C5, C7 and C10 are shown in Figure 5-20 for $0 \mathrm{~V}$ and 100V, respectively over $0.8 \mathrm{GHz}$ to $1.5 \mathrm{GHz}$. The graphical illustrations and the data in the tables show that the quality factors are low at high frequencies.

Table 5-6 Extracted capacitances

\begin{tabular}{|c|c|c|c|}
\hline Capacitor & Capacitance at 100V & Capacitance at 0V & Tuning ratio \\
\hline $\mathrm{C} 1$ & 1.4757 & 1.4556 & 1.013809 \\
\hline $\mathrm{C} 2$ & 1.4826 & 1.455 & 1.018969 \\
\hline $\mathrm{C} 3$ & 1.5125 & 1.4842 & 1.019068 \\
\hline $\mathrm{C} 4$ & 1.7712 & 1.7219 & 1.028631 \\
\hline $\mathrm{C} 5$ & 1.5455 & 1.5368 & 1.005661 \\
\hline $\mathrm{C} 6$ & 1.4562 & 1.4364 & 1.013784 \\
\hline $\mathrm{C} 7$ & 1.651 & 1.6422 & 1.005359 \\
\hline $\mathrm{C} 8$ & 1.5456 & 1.5345 & 1.007234 \\
\hline $\mathrm{C} 9$ & 1.5799 & 1.5654 & 1.009263 \\
\hline $\mathrm{C} 10$ & 1.5596 & 1.5288 & 1.020147 \\
\hline
\end{tabular}


Table 5-7 Quality factors calculated at $0.8 \mathrm{GHz}$

\begin{tabular}{|c|c|c|c|c|c|c|c|c|c|c|}
\hline Capacitor & C1 & C2 & C3 & C4 & C5 & C6 & C7 & C8 & C9 & C10 \\
\hline Q (0V) & 145.3 & 144.1 & 143.6 & 150.2 & 160.9 & 146.8 & 149.8 & 113.6 & 162.7 & 154.4 \\
\hline Q (100V) & 134.2 & 134.5 & 136.5 & 143.8 & 148 & 136.3 & 141.9 & 106.9 & 118.5 & 143.1 \\
\hline
\end{tabular}

Table 5-8 Quality factors calculated at $1.24 \mathrm{GHz}$

\begin{tabular}{|c|c|c|c|c|c|c|c|c|c|c|}
\hline Capacitor & C1 & C2 & C3 & C4 & C5 & C6 & C7 & C8 & C9 & C10 \\
\hline Q (0V) & 42.7 & 39.1 & 46.3 & 50.4 & 39.8 & 58.3 & 44.7 & 43.7 & 51.9 & 47.1 \\
\hline Q (100V) & 41.2 & 37.7 & 44.6 & 52 & 38.4 & 56.9 & 44.2 & 42.4 & 50.8 & 45.6 \\
\hline
\end{tabular}

*** The $\mathrm{Q}$ factors for the $\mathrm{C} 7$ are at $0 \mathrm{~V}$ and $75 \mathrm{~V}$.

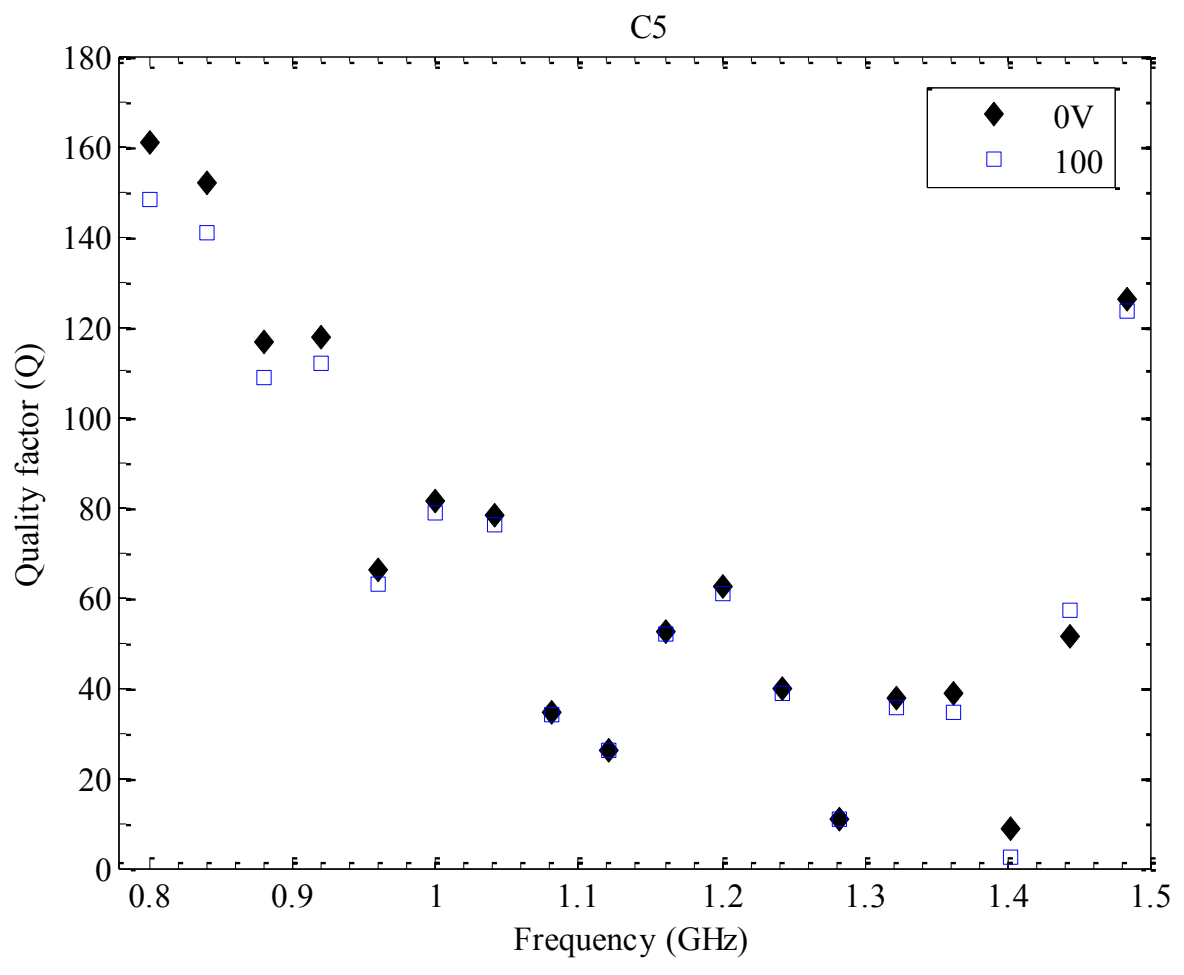

(a) 


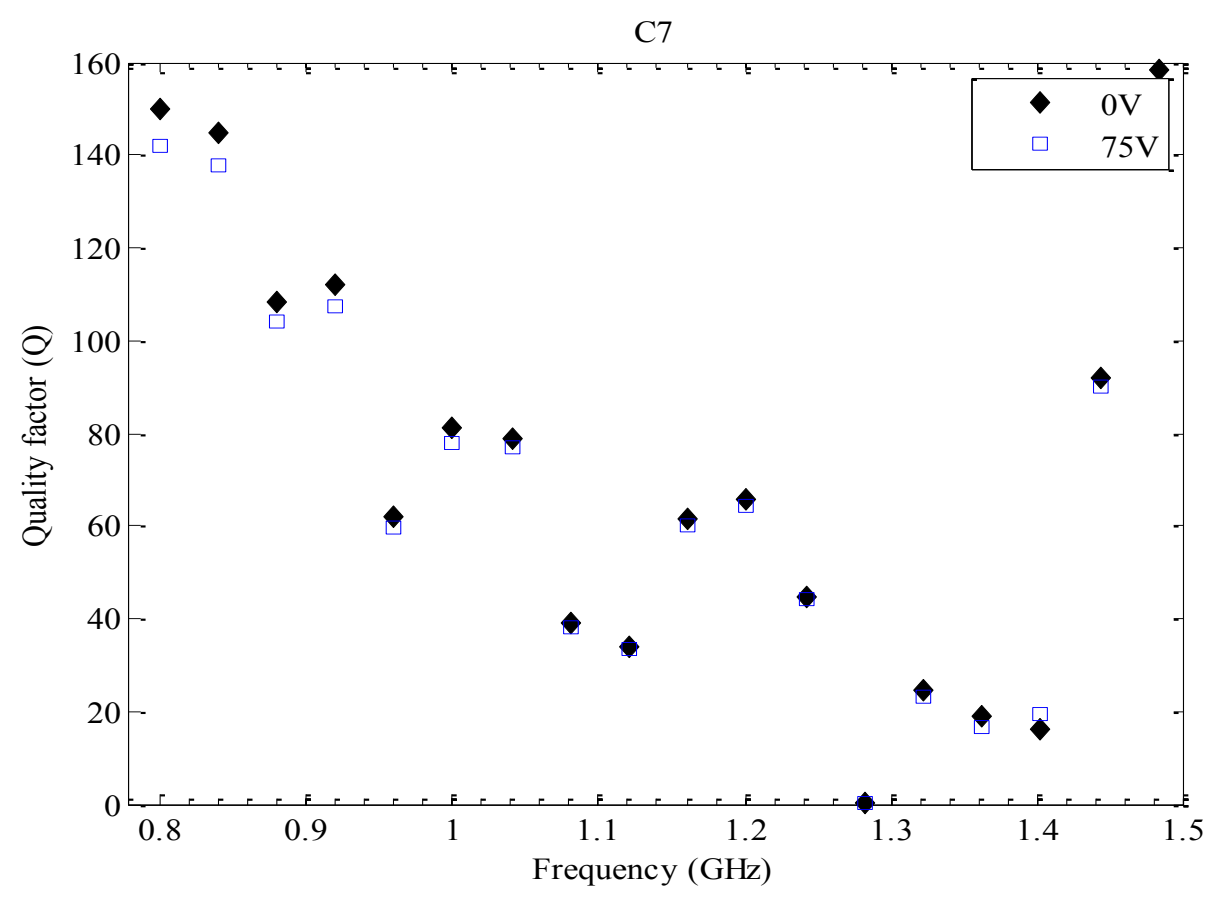

(b)

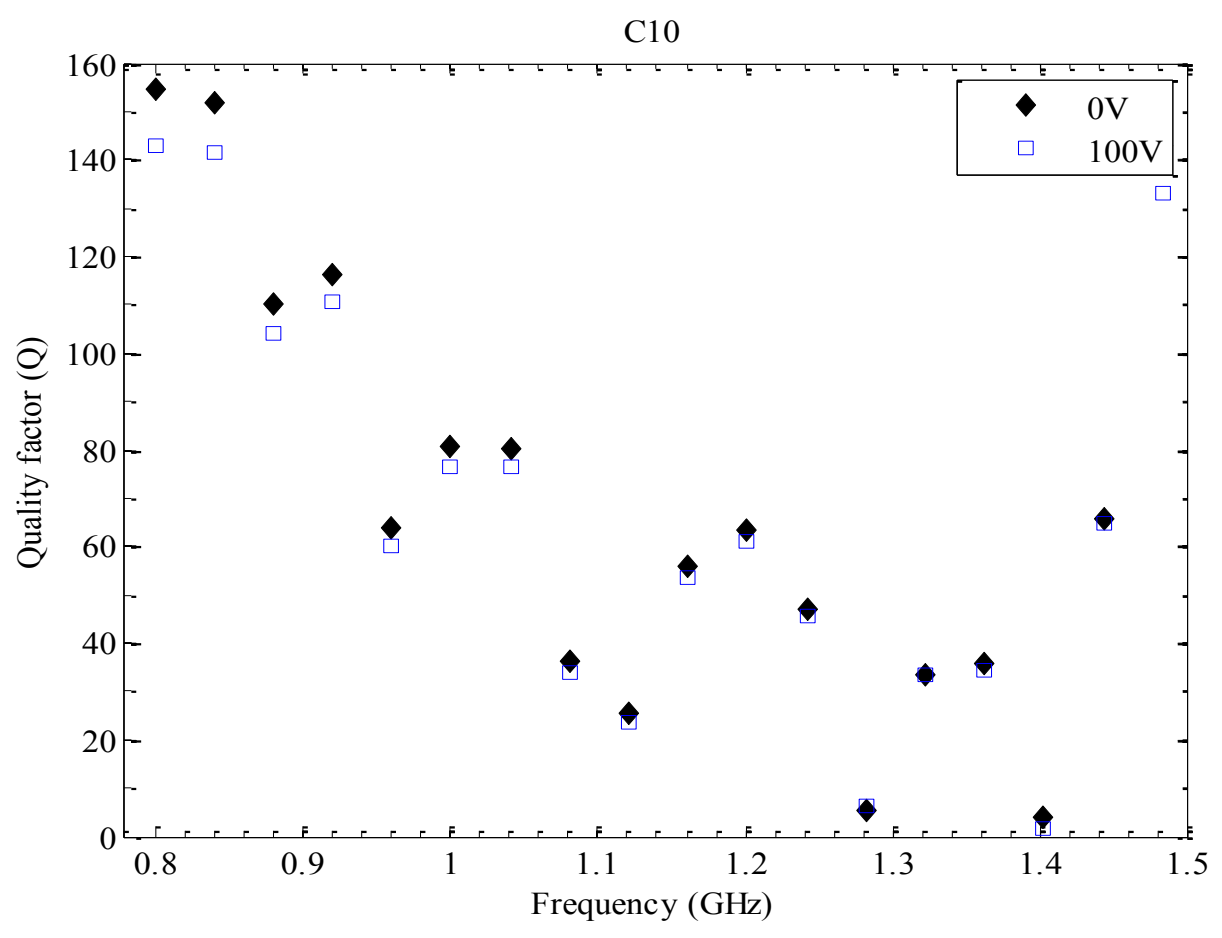

(c)

Figure 5-20 Quality factor plots of (a) C5, (b) C7 and (c) C10 
In conclusion, from the RF test results or S-parameter analyses the initial capacitances i.e. capacitances at $0 \mathrm{~V}$ which include the parasitic capacitances from substrates, dielectrics, pads, anchors, strips and the capacitance from the fingers is the most important parameter which are necessary to optimize the designs for high tuning ratio. By knowing the initial capacitance, the tuning ratio of optimized designs can be obtained accurately.

\subsubsection{Parasitic capacitance estimation}

The S-parameter measurement experiment helps to estimate the parasitic capacitance, losses in the substrate and losses in metallic strips. Substrate losses obtained through simulation and calculation result in inaccurate results because at RF frequencies capacitive and inductive losses are better characterized through measurements. However, based on the s-parameters measurement the parasitic capacitance and the losses in the substrate can be estimated. Using simulations the capacitance between the fingers, including the fringing capacitance, can be calculated. By comparing this result with the extracted capacitances, the parasitic capacitance can be accurately calculated. The results obtained in such a way can be used to get optimum performances of the capacitors such as the tuning ratio and the quality factor. The change of the number fingers and the increase in the length of the fingers would not significantly change the parasitic capacitance with other structures such as the curved beams, springs, pad sizes and strips unchanged.

\subsection{RF performance at higher actuation voltages $(\mathrm{V}>100 \mathrm{~V})$}

In section 5.7.4, the S-parameters were measured at a maximum actuation voltage of $100 \mathrm{~V}$. This actuation voltage $(100 \mathrm{~V})$ corresponds to the maximum voltage that the bias-tee can withstand to protect the vector network analyzer from high voltage. As a result, lower tuning ratios were obtained in the analysis of the capacitance (Table 5-6). The quality factors at high voltages $>100 \mathrm{~V}$ thus were not determined from the corresponding S-parameters. High quality factors are usually obtainable at lower actuation voltages and low frequency. The quality factor analysis for C1-C10 showed that the Q factors are high at 0V (Figure 5-20) and low at higher actuation voltage. However, the tuning ratios are increase at high actuation voltages. Hence, higher capacitance is achievable at only the highest displacement. 
As the electromechanical performance measurements i.e. displacements measured at actuation voltages from $0 \mathrm{~V}-187.5 \mathrm{~V}$ (Table 5-5) show the moving fingers of the capacitors achieve large displacements for voltages greater than $100 \mathrm{~V}$. However, the S-parameters were not measured because of device scarcity (bias-tee which handles voltages $>100 \mathrm{~V}$ ). The average displacement at $100 \mathrm{~V}$ is $2.1314 \mu \mathrm{m}$. Hence, the capacitance (tuning) ratios are small at $100 \mathrm{~V}$. Maximum tuning ratio is expected when the moving fingers completely move down and completely overlap with the fixed fingers.

\subsection{RF power loss}

The power dissipated in the capacitors is given by the equation:

$$
\text { Power loss }=1-\left|S_{11}\right|^{2}-\left|S_{21}\right|^{2}
$$

$S_{11}$ is the reflection coefficient at the input port and $S_{21}$ is the transmitted power from input port to the output port. The power loss includes losses in the micro-strip lines, pads, dielectrics, capacitor fingers and the substrate. It can be calculated using the measured S-parameters [111].

The power loss (in decibel) calculated from the S-parameter data are presented in graphs of Figure 5-21. The graphs show the power loss at $0 \mathrm{~V}$ and $100 \mathrm{~V}$ driving voltages. From the graphs the power increases rapidly for frequencies above $6 \mathrm{GHz}$ and attains a loss greater than $-2 \mathrm{~dB}$. 

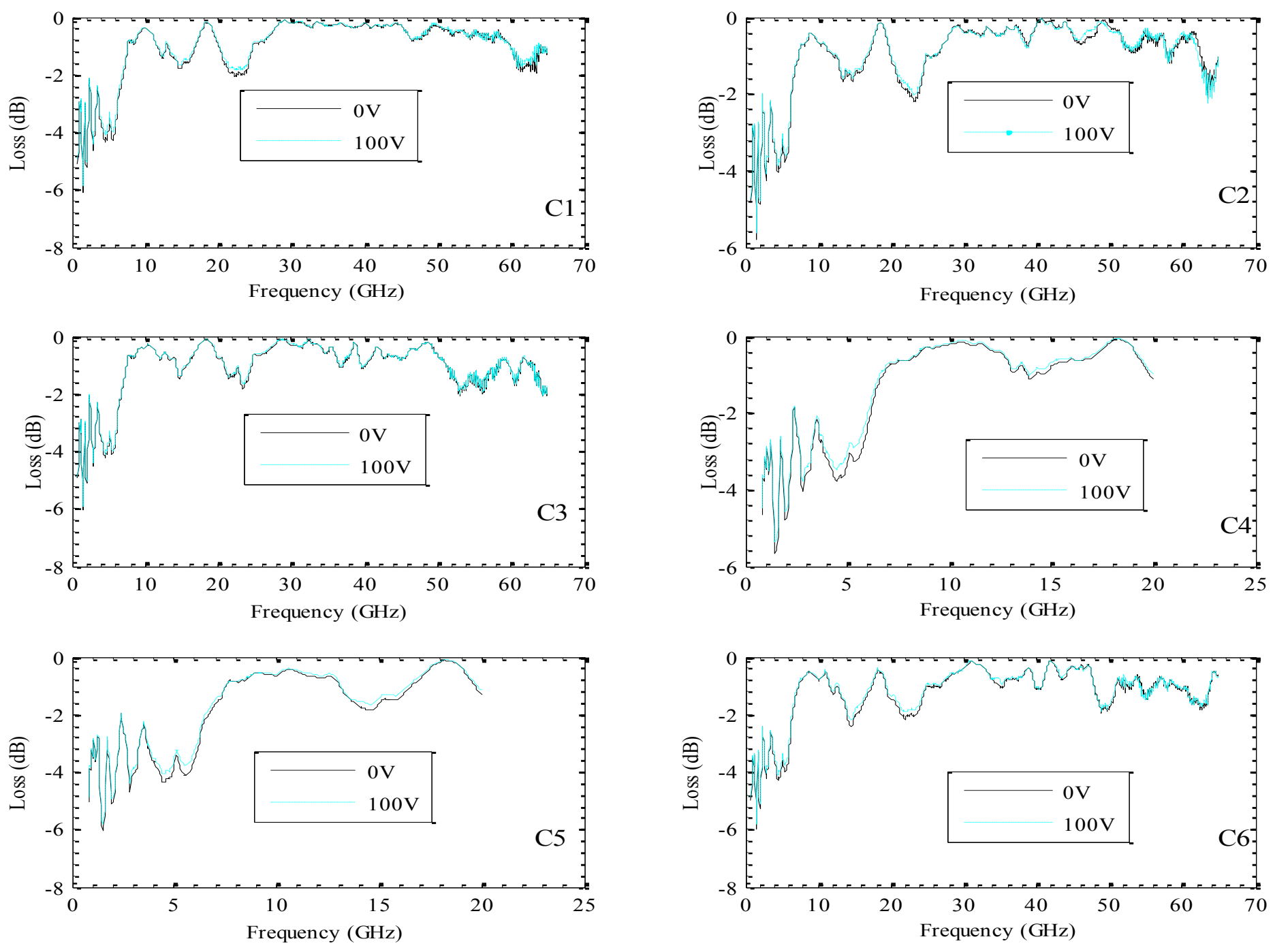

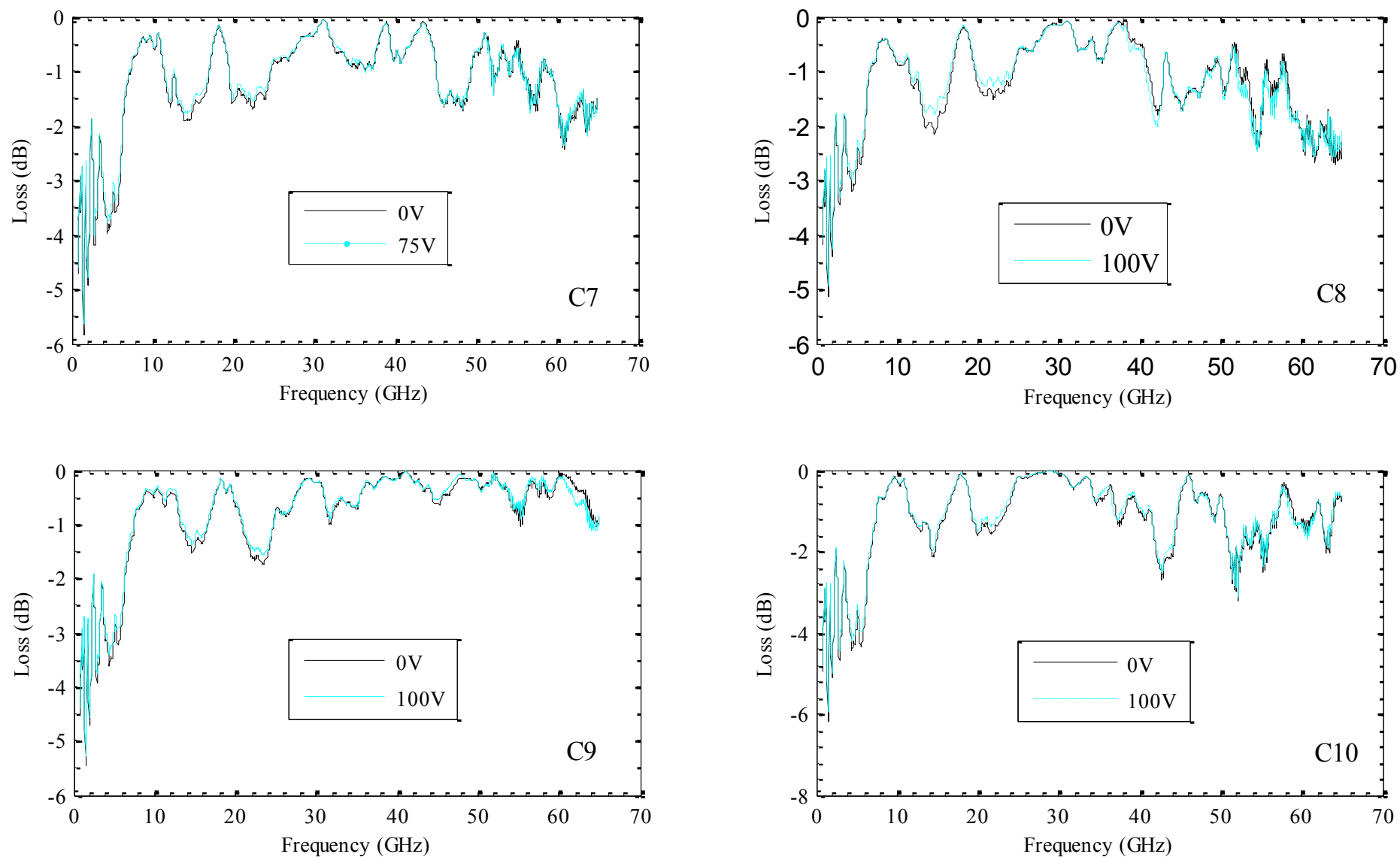

Figure 5-21 Power loss in capacitors 


\subsection{RF power handling properties}

The RF signal power induces an equivalent DC voltage on the capacitor fingers. This power adds extra electrostatic force on the capacitors. The instantaneous RF voltage is given as

$$
v_{R F}(t)=v_{\max } \sin (\omega t)
$$

where $v(t)$ is the instantaneous RF voltage at time $t, v_{\max }$ is the amplitude of the RF signal, and $\omega$ is the frequency of the signal. The RF signal induces a voltage (root mean square (RMS)) on the RF electrode of the tunable capacitor.

$$
V_{R M S}=v_{\max } / \sqrt{2}
$$

For an $\mathrm{RF}$ power $\mathrm{P}$ and characteristic impedance of the strips $\mathrm{Z}_{0}$, the equivalent root mean square voltage is given by [22];

$$
\begin{gathered}
V_{r m s}=\sqrt{P Z_{0}} \ldots \ldots \ldots . . . \\
v_{\max }=\sqrt{2 P Z_{0}} \ldots \ldots \ldots . \\
v_{R F}(t)=\sqrt{2 P Z_{0}} \sin (\omega t)
\end{gathered}
$$

Then the electrostatic force on the fingers of the capacitance can be expressed as;

$$
F_{R F}=\frac{n v_{R F}^{2}(t)}{2} \frac{d C}{d z}
$$

where, $F_{R F}$ electrostatic force due to the RF signal, $n$ the number of fingers, $v_{R F}$ instantaneous RF voltage, $P$ the RF power, $C$ capacitance, $Z_{0}$ characteristic impedance, and $z$ is the displacement.

$$
F_{R F}=\frac{n\left(2 P Z_{0} \sin ^{2} \omega t\right)}{2} \frac{d C}{d z}
$$




$$
\begin{aligned}
& F_{R F}=\frac{n}{2}\left(2 P Z_{0}\left[\frac{1}{2}-\frac{1}{2} \cos 2 \omega t\right]\right) \frac{d C}{d z} \\
& F_{R F}=\frac{n}{2}\left(\frac{1}{2} v_{\text {max }}^{2}(1-\cos 2 \omega t)\right) \frac{d C}{d z}
\end{aligned}
$$

The total electrostatic force including the DC voltage is;

$$
F=\frac{n}{2}\left(\frac{V^{2}}{2}+\frac{v_{\max }^{2}}{2}(1-\cos 2 \omega t)\right) \frac{d C}{d z}
$$

From eqn. (5.13) the DC components of the RF signal is superimposed on the DC actuation voltage and thus the net voltage across the fingers of the capacitor is $\mathrm{V}(\mathrm{DC})+\mathrm{V}(\mathrm{RF})$. When the DC voltage is zero, the only source of the electrostatic force is the RF signal power. The capacitor structure does not respond to component $1-\cos 2 \omega t$ since its mechanical resonant

frequency is less than the RF signal frequency. The RMS voltage $\geq 50 \mathrm{~V}$ can pull the moving fingers by $0.88 \mu \mathrm{m}$. The RMS voltage of $50 \mathrm{~V}$ is equivalent to $47 \mathrm{dBm}(50 \mathrm{~W}) \mathrm{RF}$ signal power.

In the experimental test of the capacitors the actuation voltage ranges were 0-200V. For RMS voltages comparable to the actuation voltages the RF signal actuates the capacitors. Therefore, the capacitors have high RF power handling capability.

\subsection{Substrate trench effects and power loss}

Thin substrates induce higher RF power loss compared with thick substrates. The addition of trenches in substrates can lower the power losses and the parasitic capacitances. Trends such as using low dielectric constant substrates are employed to improve the power loss and minimize the parasitic capacitances. In case of MetalMUMPs process, the trench in the substrate can be used to reduce the substrate power losses and minimize the parasitic capacitance. After the capacitors are fabricated two dielectric layers; a trench of $25 \mu \mathrm{m}$ depth and the silicon substrate remain. The air gap between the moving fingers and the surface of the trench is the sum of the depth of the trench and the value of the measured $H_{\max }$. The trench effect on the parameters mentioned above is compared for capacitors with and without trenches. Table 5-9 shows the capacitors with trench under the moving fingers, fixed fingers and the moving plates of the capacitors $\mathrm{C} 2, \mathrm{C} 8, \mathrm{C} 9$ and $\mathrm{C} 10$. The first three capacitors have same substrate area under the 
moving plates, the fixed fingers and the moving fingers. $\mathrm{C} 2$ has trench in the substrate where as C8 and C9 have no trench in the substrate. The graph in Figure 5-22 shows that the loss in C2 is lower compared with $\mathrm{C} 8$ and $\mathrm{C} 9$ over $0.8-40 \mathrm{GHz}$ frequency. The power loss increases for $\mathrm{C} 2$ for higher frequencies above $40 \mathrm{GHz}$. The power losses are higher for smaller trench areas (e.g. curve $\mathrm{C} 10)$ and decrease with large trench area.

Table 5-9 Areas of trenches under the capacitor fingers and plates

\begin{tabular}{cccc}
\hline Capacitor & Area with trench $\left(\mu \mathrm{m}^{2}\right)$ & Area without trench $\left(\mu \mathrm{m}^{2}\right)$ & Trench \\
\hline C2 & $1050 \times 774$ & ------ & Yes \\
C8 & ----- & $1050 \times 774$ & No \\
C9 & ------ & $1050 \times 779$ & No \\
C10 & $770 \times 788$ & ------ & Yes \\
\hline
\end{tabular}

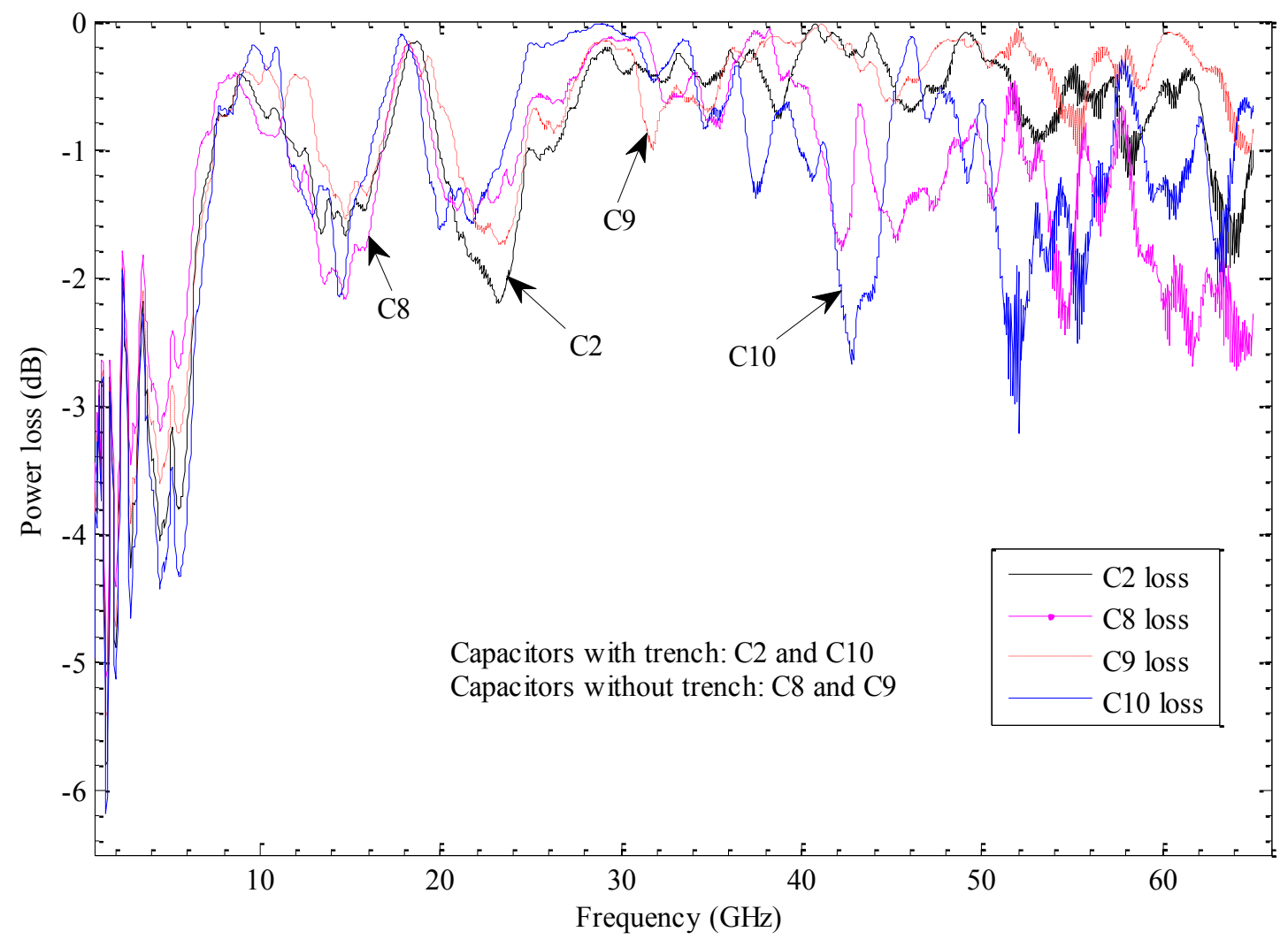

Figure 5-22 Power loss with and without trench 


\subsection{Chapter summary}

Tunable capacitors with high quality factors were designed using stress gradient based vertical comb-drive actuators. The curved beams and the anchoring springs of the actuators developed in Chapter 4 were modified to increase the deflection by lowering the stiffness. Larger numbers of fingers were added to increase the electrostatic force of attraction. Simulations using the residual stress gradient were studied to determine the deflection of the modified actuator. Capacitors using the new curve-up beams and actuators were fabricated and tested to verify the simulated results. Ten capacitors were tested and the displacements measured under DC actuation voltages were presented. Higher displacements have been achieved by the modified design of the actuator over $0-200 \mathrm{~V}$. Higher displacements were obtained at comparatively low actuation voltages.

The RF performances of the tunable capacitors were analyzed from the scattering (S) parameter measurements. The S-parameters were used to extract the capacitance to calculate the tuning ratios and the quality factors. A Matlab program was written to extract the capacitance and the resistance from the S-parameters. High quality factors ranging from 37.7-162.7 for 0V-100V were calculated at $1.4 \mathrm{GHz}$ and $0.8 \mathrm{GHz}$ using the extracted capacitances. However, the calculated capacitance changes were very small due to high parasitic capacitance between the RF pads, anchors and strips on the substrate. The parasitic capacitances are proportional to the area of the pads, strips and the thickness of the substrate. The RF power losses in the capacitors were also analyzed from the S-parameters.

In conclusion the tuning ratios of the capacitors were small and the power losses were very high. Chapter 6 presents the optimization of the capacitors to obtain high tuning ratios. The variable optimized is the change of the capacitance $(\Delta \mathrm{C})$ which depends on the number and the length of the fingers. 


\section{Tunable capacitor optimization}

In Chapter 5 the design of residual stress gradient based comb-driven tunable capacitor had been discussed. Tuning ratios and quality factors had been extracted from the measured S-parameters. However, the capacitors needed high actuation voltages for operation and the tuning ratios obtained were low. It was also observed that the substrate parasitic capacitances are much higher than the actual tunable capacitance. In this Chapter the tunable capacitor is optimized to obtain better tuning ratio and quality factor. Due to small capacitance change and parasitic capacitances high tuning ratios were not obtained. Two optimization techniques are pursued in this chapter. The parameter that is optimized is the change of capacitance at any actuation voltage when the moving fingers move towards the fixed finger. In the first optimization technique high tuning ratios are obtained by increasing the number and the length of fingers with minimum gap between fingers. Using large number of fingers increases the total capacitance between the moving and the fixed fingers. At the time of actuation, when the moving fingers move towards the fixed fingers the change in the capacitance also increases. Minimizing the horizontal gap between the moving fingers and the fixed finger shortens the fringing fields. Thus, the capacitance density will be increased. Minimum gap in conjunction with long fingers improves the tuning ratio significantly. Simulations are used to accurately determine the capacitances between the fingers without the substrate.

Parasitic capacitance reduction and minimization of the power losses from RF strips, pad sizes and anchor area to the substrate are found to be the second techniques to improve the tuning ratios and the quality factors. Even though it is difficult to determine the substrate losses or parasitic capacitance, the experimental results of the tunable capacitor $\mathrm{C} 7$ are used as a level of comparison for the optimization. The measured capacitance of $\mathrm{C} 7$ at $0 \mathrm{~V}$ includes the capacitance of the fingers and the parasitic from the substrate. By subtracting the simulated fingers capacitance from the measured capacitance of $\mathrm{C} 7$ at $0 \mathrm{~V}$ the parasitic capacitance is obtained. The next sections discuss the optimization techniques in detail. The methods used to optimize the tuning ratio combine accurate simulation results and experimental results of $\mathrm{C} 7$. 


\subsection{Increasing tuning ratio}

Tuning ratio of a tunable capacitor is the ratio of maximum capacitance to minimum capacitance. The maximum capacitance occurs when the moving fingers travel down towards the fixed fingers for non-zero actuation voltages. The minimum capacitance refers to the initial capacitance, i.e. the capacitance value at zero volts.

\subsubsection{Idea of increasing tuning ratio}

The capacitance between a comb structure increases with the number of fingers and the length of fingers. At a given voltage the electrostatic force between the moving and the fixed fingers of the capacitors can be increased.

The tuning ratio of a tunable capacitor is given by the equation:

$$
T R=\frac{C_{0}+\Delta C}{C_{0}}
$$

$C_{0}$ represents the initial capacitance or the minimum capacitance measured at $0 \mathrm{~V}$ while $\Delta C$ stands for the change of capacitance when actuation voltage $(\mathrm{V} \neq 0)$ is applied. $\Delta C$ is the change of capacitance that occurs when the moving fingers travel a certain displacement due to the applied actuation voltage. High $\Delta C$ values result in high tuning ratios. The value $C_{0}$ is a constant value which exists when the actuation voltages change. The change in capacitance, $\Delta C$, can be increased by using long fingers, large number of fingers (n), narrowing the gap $(d)$ between the fixed and the moving fingers. Symbolically,

$$
\Delta C=f\left(L_{f}, n, d\right)
$$

Increasing the number of fingers raises the electrostatic attraction force between the fingers of the capacitor. The force is proportional to the square of the actuation voltage $(\mathrm{V})$, the number $(n)$ and the length $\left(L_{f}\right)$ of capacitor fingers.

$$
F_{e} \propto V^{2}, n, L_{f}
$$

As the electrostatic force increases with increased length of the fingers, the vertical displacement the moving fingers travel increases significantly. As a result the change of the capacitance for large electrostatic force is high. 


\subsubsection{Optimization of finger size, number and gap}

Optimization of the dimensions of fingers and the number of fingers are the basic strategies to obtain high tuning ratio. It was found out that long fingers provide high electrostatic forces and high capacitance. However, due to the stress gradient of the MetalMUMPs fabrication the length of the fingers could not be arbitrarily selected. The bending of the fingers is more prominent for very long fingers. For extremely long fingers the stress gradient curves the fingers and thus the fingers could touch the substrate. For instance, from the experimental measurement of $\mathrm{C} 7$ in Chapter 5, a $500 \mu \mathrm{m}$ long finger achieved $2.8 \mu \mathrm{m}$ bending at its tip. The parts of the moving fingers lie below the top surface of the fixed fingers. The bending of the fingers slightly reduces the electrostatic forces. Ideally planar surfaced fingers produce the highest electrostatic forces. The finger length selection for the optimized design is based on the simulation of the curving induced by the stress gradient. Finger lengths that give moderate bending are chosen.

The moving fingers of the capacitors are mainly affected by the curving because these fingers are suspended above the substrate. Similarly the fixed fingers are subject to bending due to the stress gradient. However, it is possible to prevent the curving of the fixed fingers by the substrate underneath. The bending of fingers with lengths from $100 \mu \mathrm{m}$ to $1000 \mu \mathrm{m}$ is simulated using the residual stress gradient and the plot of tip bending vs. finger length is illustrated in Figure 6-1.

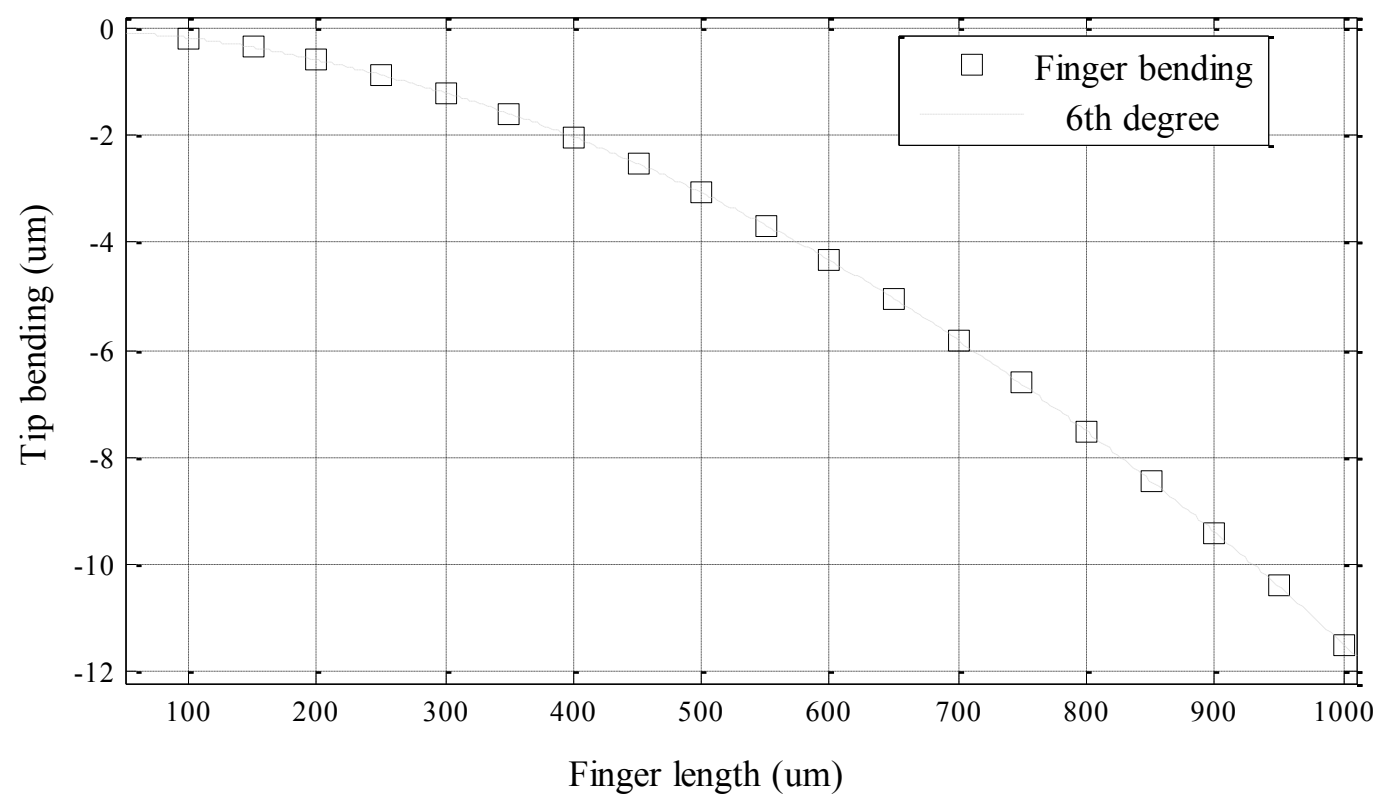

Figure 6-1 Finger tip bending vs. length of fingers 
Figure 6-2 shows the curving of the moving fingers and the fixed fingers. The curving of the fingers causes misaligned surfaces between the fingers i.e. the bottom or top surfaces of the fingers will not remain in the same plane. The misalignment is more sever for longer fingers.

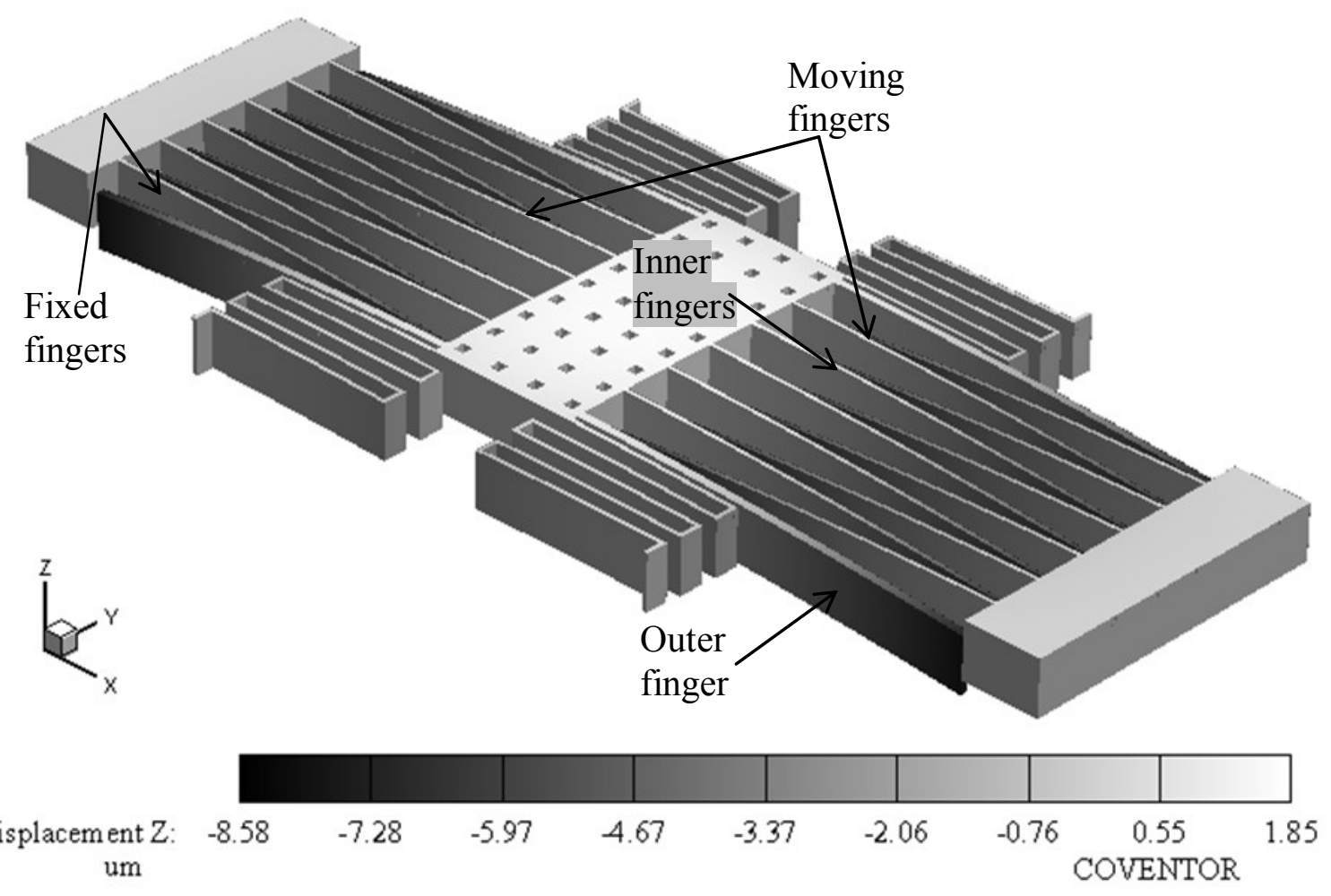

Figure 6-2 Residual stress gradient bent fingers

On the other hand the numbers of fingers are selected using the available space on the length of the moving plate of $\mathrm{C} 7$. Based on the fabrication process limits, i.e. the minimum feature sizes allowed, the number fingers can be fixed. According to the MetalMUMPs process, the minimum finger width is $8 \mu \mathrm{m}$ and the gap between two fingers is $8 \mu \mathrm{m}$. The numbers of fingers that can be put on the moving plate of $\mathrm{C} 7$ are 20 .

\subsubsection{Influence of electrostatic forces on the outer fingers}

Experimental analysis of the electrostatic force on the outer fingers of the capacitors was visually inspected using the microscope used for testing. It was observed that the outer moving fingers of the capacitor stick to the neighboring fixed fingers when the actuation voltages increased. The inner fingers and the outer fingers are labeled as shown in Figure 6-3. 


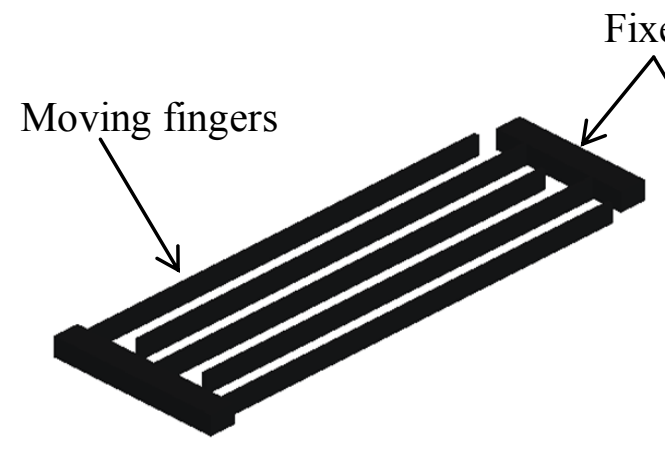

(a)

Fixed fingers

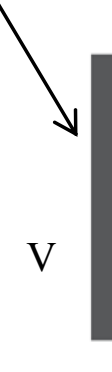

)

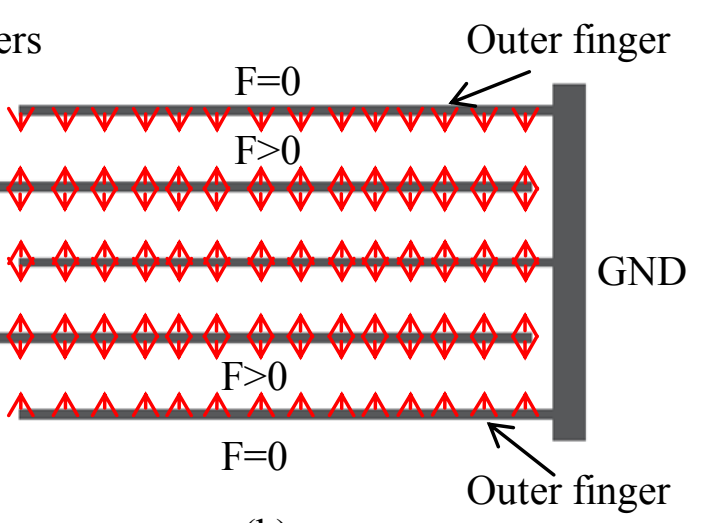

(b)

Figure 6-3 Moving fingers and fixed fingers of the capacitor (a) and distribution of electrostatic force on the side walls of the fingers (b)

The electrostatic force on the side walls of the moving and the fixed fingers are balanced for inner fingers under ideal conditions. However, a close look on the most outer moving fingers shows that only one-sided force exists on these fingers. This situation causes the outer moving fingers to easily stick onto the fixed fingers for long fingers $\geq 400 \mu \mathrm{m}$ and narrow gap. Experimental measurement showed that fingers of length $>400 \mu \mathrm{m}$ could be easily snap to the fixed fingers. To prevent the stiction the outer fingers need to be stiff enough to resist the pulling force from the sidewall of the fixed fingers. This can be done by reducing the length of the fingers or increasing the width of the fingers. The former method however lowers the electrostatic force on the fixed finger, capacitance will be reduced and unbalanced forces occur on the fixed finger. The second method increasing the width of the fingers does not affect the electrostatic force and it maintains the capacitance. Figure 6-3 (a) shows the arrangement of the moving fingers and the fixed fingers. In this illustration the fixed fingers are bounded by the moving fingers. Figure 6-3 (b) shows the electrostatic force distribution when a voltage $\mathrm{V}$ is applied to the fixed fingers. A single inner finger experiences two opposite forces that act on its side walls. The arrows show the directions of the electrostatic forces on the side walls of the inner fingers. The forces on the inner fingers have magnitudes of $F>0$ for $V \neq 0$. Since the forces on the side walls of the inner fingers are opposite and equal for identical voltages (V), the fingers remain in equilibrium state. On the other hand, the force on the side wall of the outer moving 
finger is zero $(\mathrm{F}=0)$. Therefore, the forces acting on the inner sides pull these fingers and stick on to the walls of the fixed fingers.

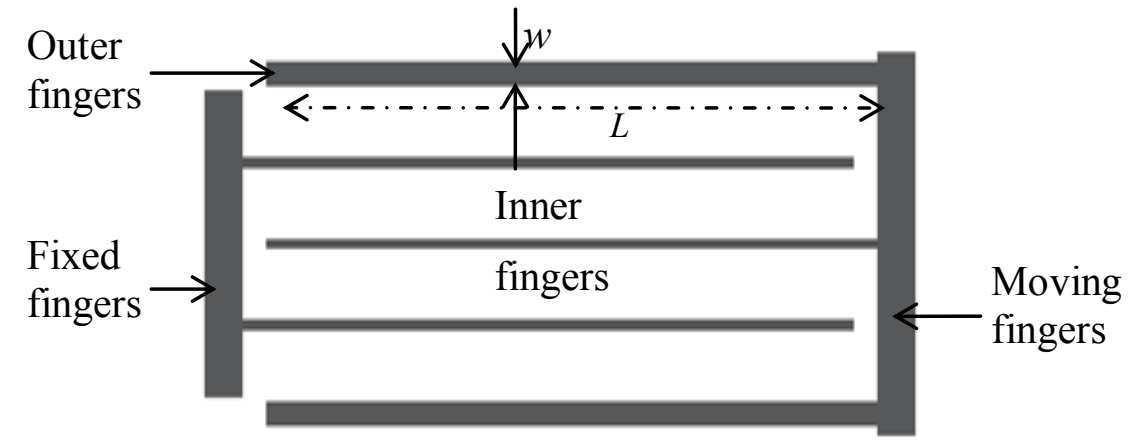

Figure 6-4 Wider outer moving fingers

The method proposed at the beginning of this section is applied to reduce the stiction of the outer moving fingers from the fixed fingers. Figure 6-4 illustrates the method used to eliminate the stiction problem by using wider outer fingers. The width of the outer moving fingers is larger than the width of the inner fingers. The stiffness of the fingers increases with the width according the equation [115];

$$
K=E t\left(\frac{w}{L}\right)^{3}
$$

where $K=$ the spring constant, $E=$ the Young's modulus, $t=$ the thickness, $w=$ the width and $L=$ the length of the fingers. The electrostatic force between the moving and the fixed fingers is given by;

$$
F_{e}=n \varepsilon V^{2} \frac{w}{d}
$$

$F_{e}$ the electrostatic force of attraction, $n=$ the number of fingers, $V$, the applied voltage, $\varepsilon$ is the dielectric constant and $d$ is the gap between moving and fixed fingers. The maximum displacement, $x$, of the outer moving finger is given by;

$$
x=\frac{n \varepsilon w}{K d} V^{2}
$$


However, the Equation (6.5) does not properly include the fringing effects from the top surface of the moving fingers and the fixed fingers. As a result the calculated values of the electrostatic forces and the displacement largely deviate from the simulated results. In this section electrostatic simulation results are preferred. In the analysis one moving finger and one fixed finger are sufficient for the analysis side pulling effect. Based on the lateral displacement of the moving finger, the width of the finger is adjusted and the value that ensures no side pull-in is selected. The two assumptions on which the analysis of the lateral displacement based are (1) the side walls of the moving and the fixed fingers form boundaries of a parallel plate capacitor and (2) the side wall of the fixed finger is in equilibrium (i.e. static), the moving finger can be modeled as simple cantilever beam. Therefore, the lateral pull-in effect on the tip of the moving finger is the variable to analyze the stiction problem.

CoventorWare simulations are run to determine the conditions of the pull-in effect. For a gap of $9 \mu \mathrm{m}$, the pull-in state occurs after $3 \mu \mathrm{m}$, which is one-third of the original gap. Table 6-1 shows the summary of the simulated lateral pull-in voltages and the displacement of the tips of the moving fingers. The widths of the outer moving fingers are varied from $9 \mu \mathrm{m}$ to $12 \mu \mathrm{m}$ for finger length of $800 \mu \mathrm{m}$. The minimum voltage applied to avoid the stiction problem for a $9 \mu \mathrm{m}$ wide finger is $97.5 \mathrm{~V}$. When the width further increases to $15 \mu \mathrm{m}$ the pull-in voltage increases which ensures that the fingers reach sufficient stiffness that maintains stability at the maximum actuation voltage. In conclusion, $800 \mu \mathrm{m}$ long and $16 \mu \mathrm{m}$ wide outer fingers provide sufficient stiffness to prevent the stiction.

Table 6-1 Optimal capacitor dimensions

\begin{tabular}{|c|c|c|c|c|c|c|c|c|}
\hline \multicolumn{2}{|c|}{ Finger width } & \multicolumn{2}{|c|}{ Finger length } & \multicolumn{2}{|c|}{ Number of fingers } & \multirow[t]{2}{*}{ Gap } & \multirow{2}{*}{$\begin{array}{l}\text { Thickness } \\
\text { of fingers }\end{array}$} & \multirow{2}{*}{$\begin{array}{l}\text { Width of outer } \\
\text { moving fingers }\end{array}$} \\
\hline Moving & Fixed & Moving & Fixed & Moving & Fixed & & & \\
\hline 8 & 8 & 800 & 800 & 20 & 19 & 8 & 20.5 & 16 \\
\hline
\end{tabular}

Table 6-2 summarizes the range of the length and width of the moving fingers. The corresponding pull in voltages to each of the fingers are listed in the table. Outer moving fingers with thickness greater than $13 \mu \mathrm{m}$ and length $700 \mu \mathrm{m}$ provide good stability. However, outer fingers with $800 \mu \mathrm{m}$ length and $16 \mu \mathrm{m}$ width lead to better stability and higher tuning ratio. 
Table 6-2 Summary of optimal width of the outer moving fingers

\begin{tabular}{cccc}
\hline Finger length $(\mu \mathrm{m})$ & Width $(\mu \mathrm{m})$ & Range of pull-in voltage $(\mathrm{V})$ & Displacement $(\mu \mathrm{m})$ \\
\hline 700 & 13 & $160-168.75$ & $2.86-4.04$ \\
800 & 16 & $170-176.5$ & $3.08-4.07$ \\
900 & 18 & $170-174.3$ & $3.32-4.06$ \\
\hline
\end{tabular}

\subsubsection{Tuning ratio of the optimized capacitor}

Schematic representation of the optimized tunable capacitor is shown in Figure 6-5. Using the experimental testing results of the S-parameters of the capacitor C7 the parasitic capacitance can are known. The capacitance between the moving fingers and the fixed fingers can be accurately determined using simulations. Assuming that the change of the parasitic capacitance due to the increased number of fixed and moving fingers is negligible the tuning ratio of the optimized capacitor can be calculated. The parasitic capacitance of the $\mathrm{C} 7$ can be obtained by subtracting the simulated capacitance of the fingers at $0 \mathrm{~V}$ from the extracted capacitance in Table 5-6.

$$
C_{\text {parasitic }}(C 7)=C_{0}-C_{\text {fingers }}(0 \mathrm{~V})
$$

The tuning ratio (TR) of the capacitance at any voltage is given by the equation:

$$
T R=\frac{C_{0}+\Delta C_{V}}{C_{0}} .
$$

where $C_{0}$ is the total extracted capacitance at $0 \mathrm{~V}$ and $\Delta C_{v}$ is the change in capacitance obtained at any actuation voltage $\mathrm{V}$.

The capacitance of the optimized design at $0 \mathrm{~V}$ can be calculated using the equation:

$$
\begin{aligned}
& C_{\text {optimized }}(0 \mathrm{~V})=C_{\text {parasitic }}(C 7)+C_{\text {optimized_fingers }}(0 \mathrm{~V}) \\
& C_{\text {complete_overlap_optimized }}=C_{\text {parastic }}(C 7)+\Delta C_{\text {optimized_fingers_complete_overlap }}
\end{aligned}
$$

The tuning ratio of the optimized design can be calculated using the equation:

$$
T R_{\text {optimized }}=\frac{C_{\text {optimized_complete_overlap }}}{C_{\text {optimized }}(0 \mathrm{~V})}
$$




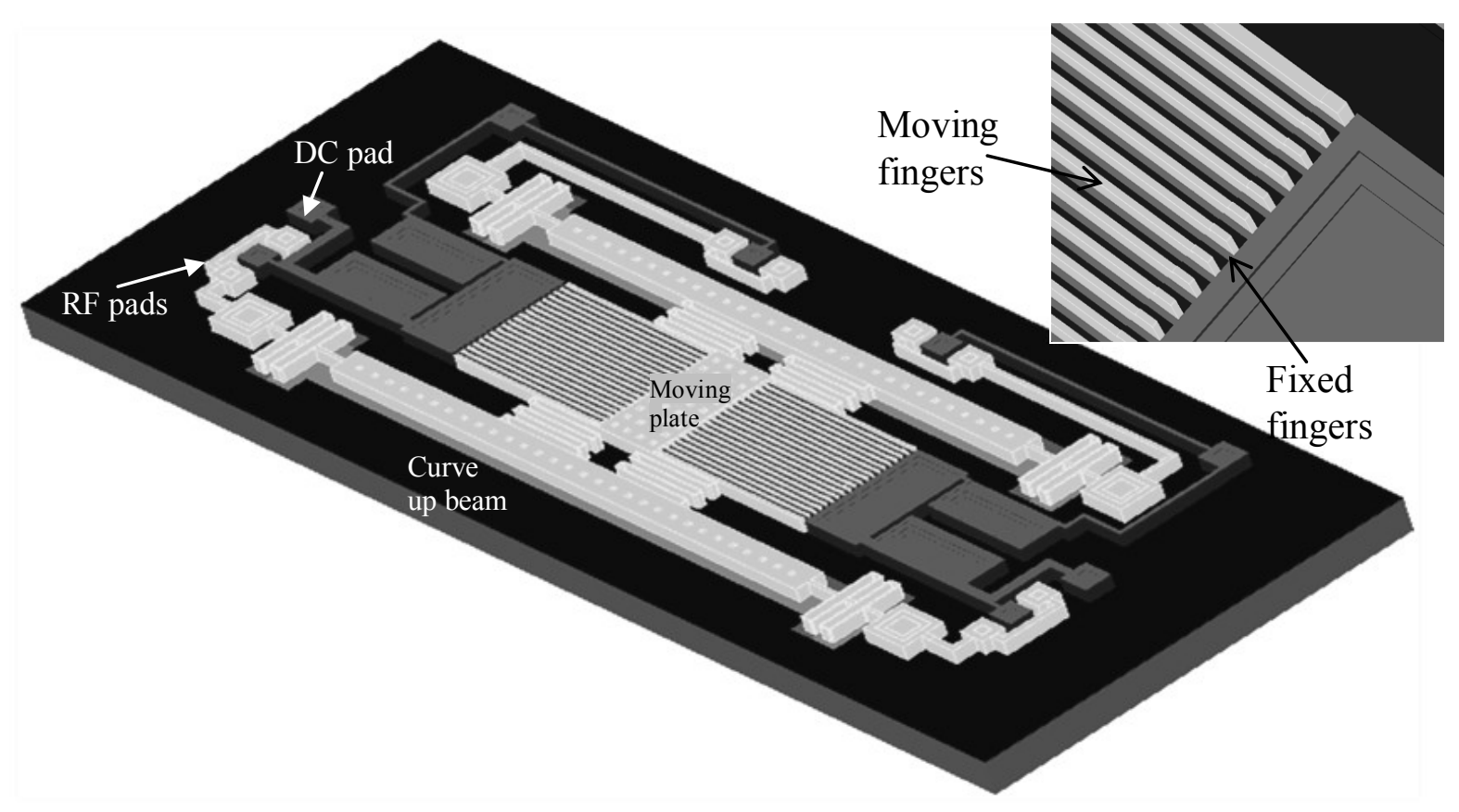

(a)

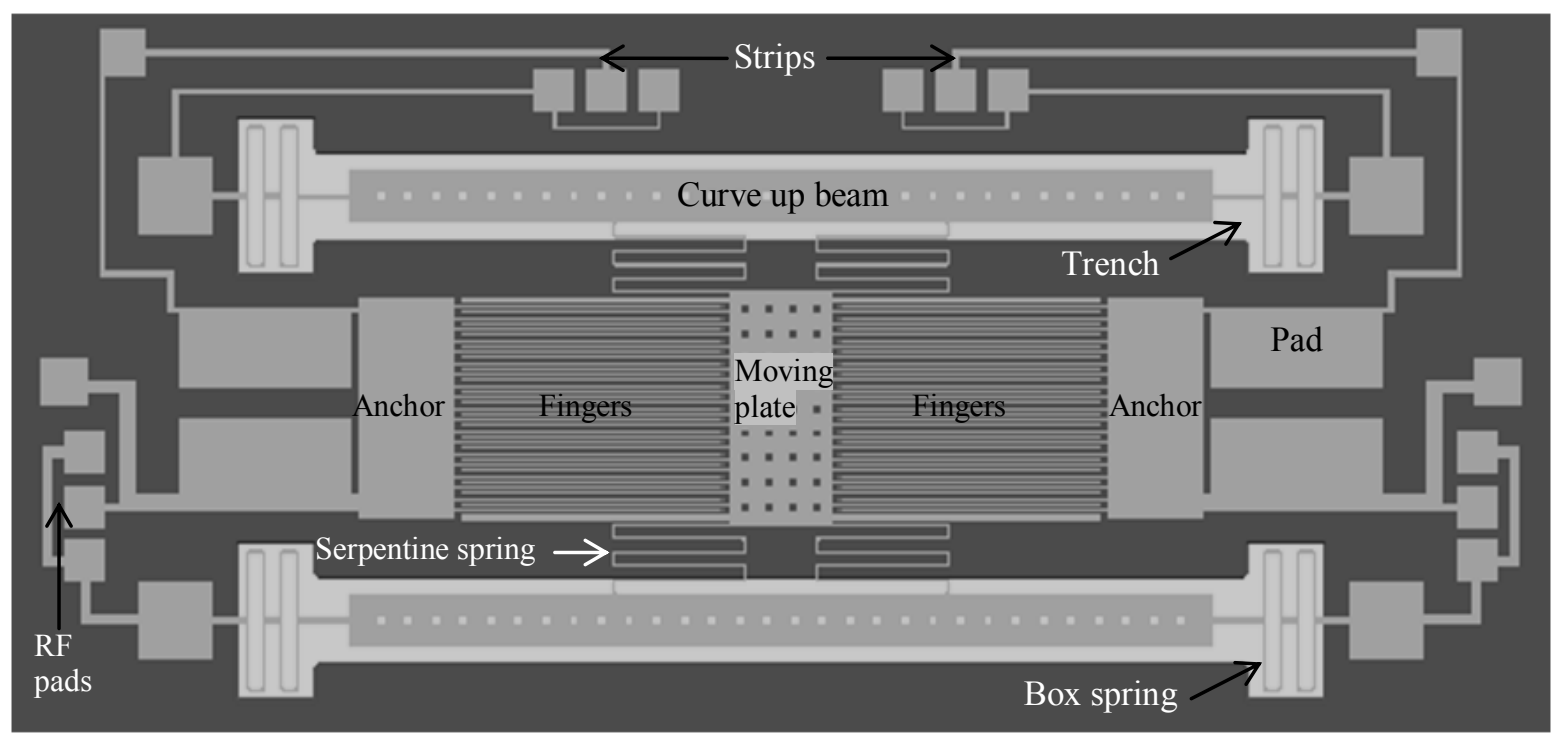

(b)

Figure 6-5 Schematic representation of optimized tunable capacitor (a) 3D view and (b) top view 
From the experimental analysis results of C7 the parasitic capacitances from the substrate can be accurately calculated. However, the actual capacitance between the fingers can be determined via simulation. The simulated capacitance at $0 \mathrm{~V}$, i.e. when the fingers are at $24.38 \mu \mathrm{m}$ above the substrate, is $264.4 \mathrm{fF}$. This capacitance is subtracted from the capacitance extracted from the Sparameters to obtain the parasitic capacitance. Using eqn. (6.7) the parasitic capacitance is found to be;

$$
C_{\text {parasitic }}(C 7)=1.6422-0.2644=1.3778 p F
$$

In Table 6-3 the simulated and the calculated capacitances are shown. The values listed in the table are calculated using equations (6.7) to (6.10). The simulated capacitances refer to the position of the moving plate and the moving fingers of the optimized capacitor. Capacitance at $24.38 \mu \mathrm{m}$ is the capacitance at $0 \mathrm{~V}$ and the capacitance at $0 \mu \mathrm{m}$ refers to the complete overlap of the moving fingers with the fixed fingers. Complete overlap is achieved at $162.5 \mathrm{~V}$. Finally the tuning ratio is calculated using equation (6.11). The tuning ratio obtained by increasing the fingers length and narrowing the gap between the fingers is $153.5 \%$.

Table 6-3 Capacitances of optimized tunable capacitor

\begin{tabular}{|c|c|c|c|c|c|}
\hline $\begin{array}{c}\text { Parasitic } \\
\text { capacitance } \\
(0 \mathrm{~V})\end{array}$ & $\begin{array}{c}\text { Simulated } \\
\text { capacitance at } \\
24.38 \mu \mathrm{m}(0 \mathrm{~V})\end{array}$ & $\begin{array}{c}\text { Simulated } \\
\text { capacitance at } \\
0 \mu \mathrm{m}(162.5 \mathrm{~V})\end{array}$ & $\begin{array}{c}\text { Capacitance } \\
\text { optimized at } \\
24.38 \mu \mathrm{m}(\text { at } 0 \mathrm{~V})\end{array}$ & $\begin{array}{c}\text { Capacitance } \\
\text { optimized at } \\
0 \mu \mathrm{m}(162.5 \mathrm{~V})\end{array}$ & $\begin{array}{c}\text { Tuning } \\
\text { ratio }\end{array}$ \\
\hline $1.3778 \mathrm{pF}$ & $0.852 \mathrm{pF}$ & $2.045 \mathrm{pF}$ & $2.23 \mathrm{pF}$ & $3.423 \mathrm{pF}$ & $153.5 \%$ \\
\hline
\end{tabular}

Optimization of the tuning ratio of the capacitance changes the quality factors of the optimized capacitor. Increasing the length of the fingers induces high resistance which reduces the quality factor. Narrowing the gap between the fingers of the capacitor also reduces the quality factor. This can be verified with using the relationship of the quality factor with the resistance and the capacitance from Equation. (6.13). R represents the resistance and $\mathrm{C}$ the capacitance

$$
Q \propto \frac{1}{R C}
$$


Due to these two increased parameters the quality factor will be lowered. At higher frequencies the quality factor further decreases. Since at microwave frequencies the surface resistivity increases due to the skin effect the resistance could not be accurately represented with lumped model equivalent circuit.

\subsection{Optimization of pads and strips}

Parasitic capacitance significantly dominates the capacitance between the moving and the fixed fingers. The metal pads, anchors and the strips produce significant capacitance through the dielectric substrates. The parasitic capacitances are bigger for large areas of the pads, anchors, the strips, thicknesses of the dielectric layers and the thickness of the substrates. The thickness $(20.5 \mu \mathrm{m})$ of the metal strips also contributes to the parasitic capacitance through fringing fields from the side walls. The fringing fields increase the mean area of covered by the aforementioned structures and worsen the parasitic capacitance. The idea of minimizing pads and anchors reduces the mean area so that the parasitic capacitance can be lowered. The pad, strip and anchor structures are illustrated in Figure 6-6 for design C7. The complete design of the optimized tunable capacitor is shown in Figure 6-7.

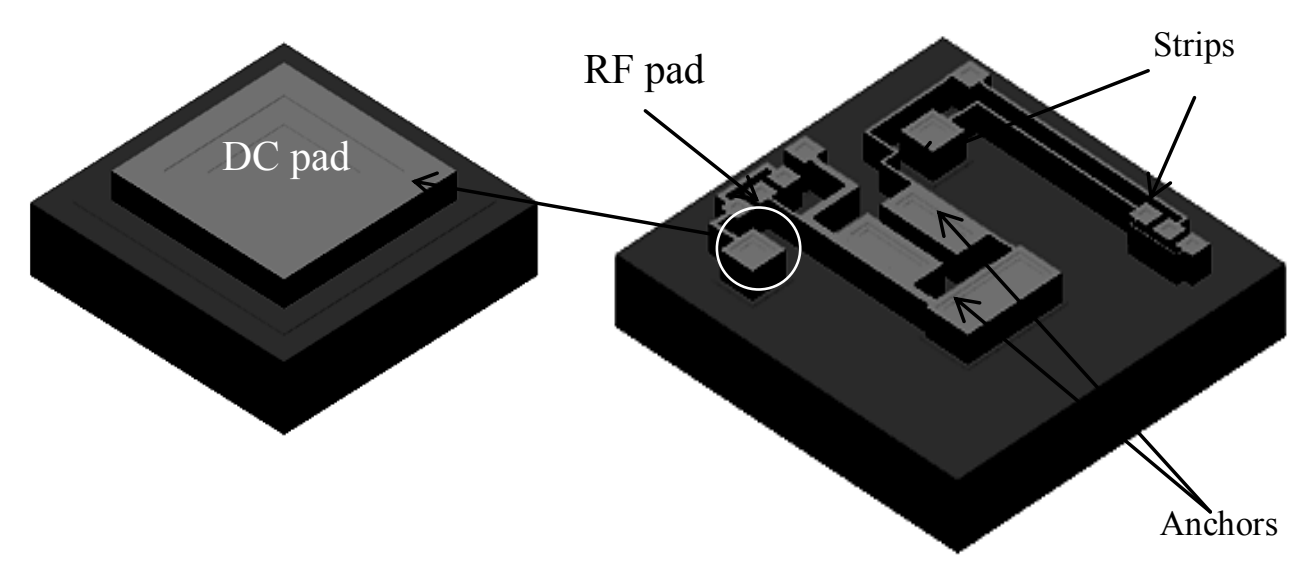

Figure 6-6 C7 pads and strips 


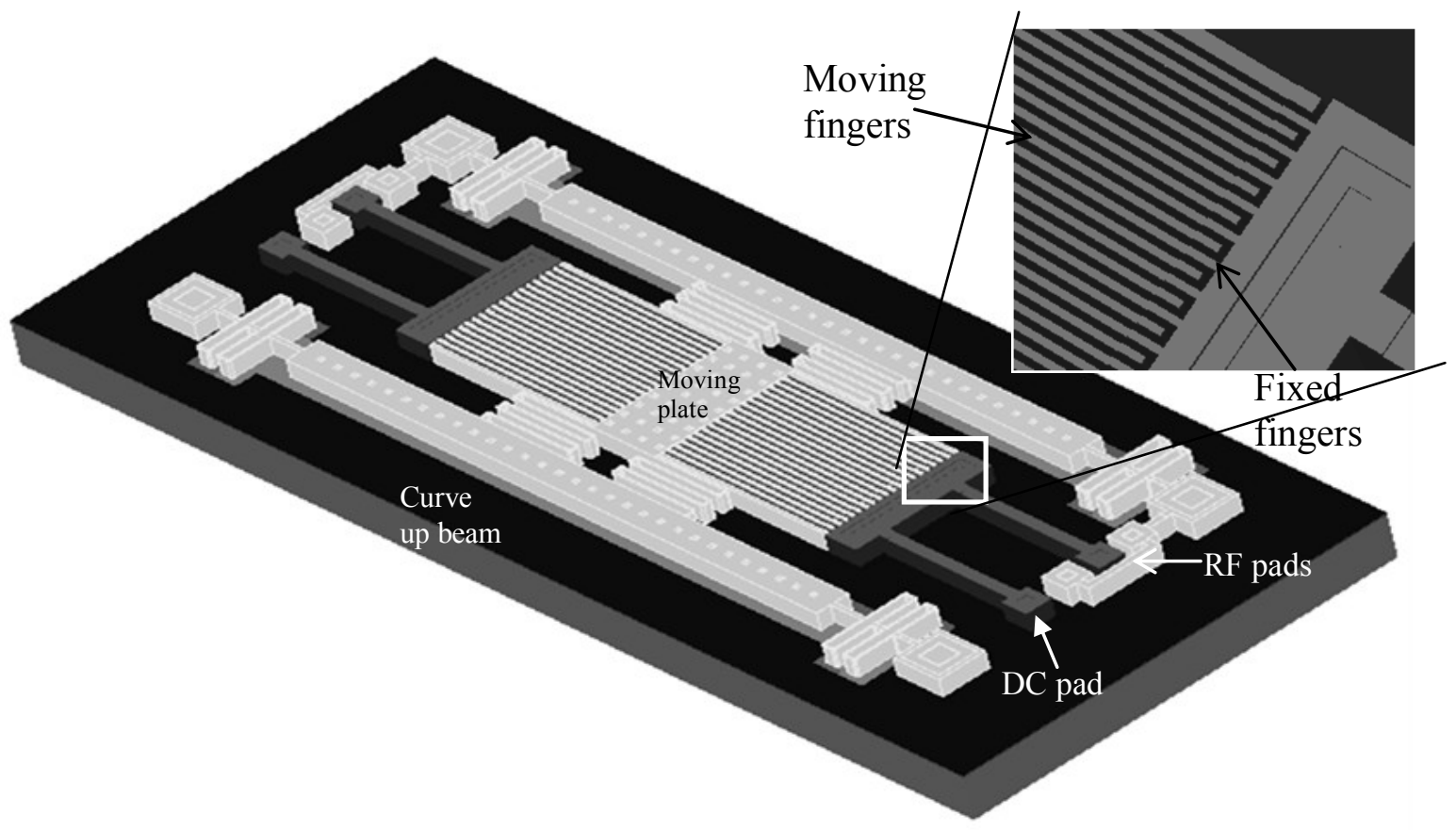

(a)

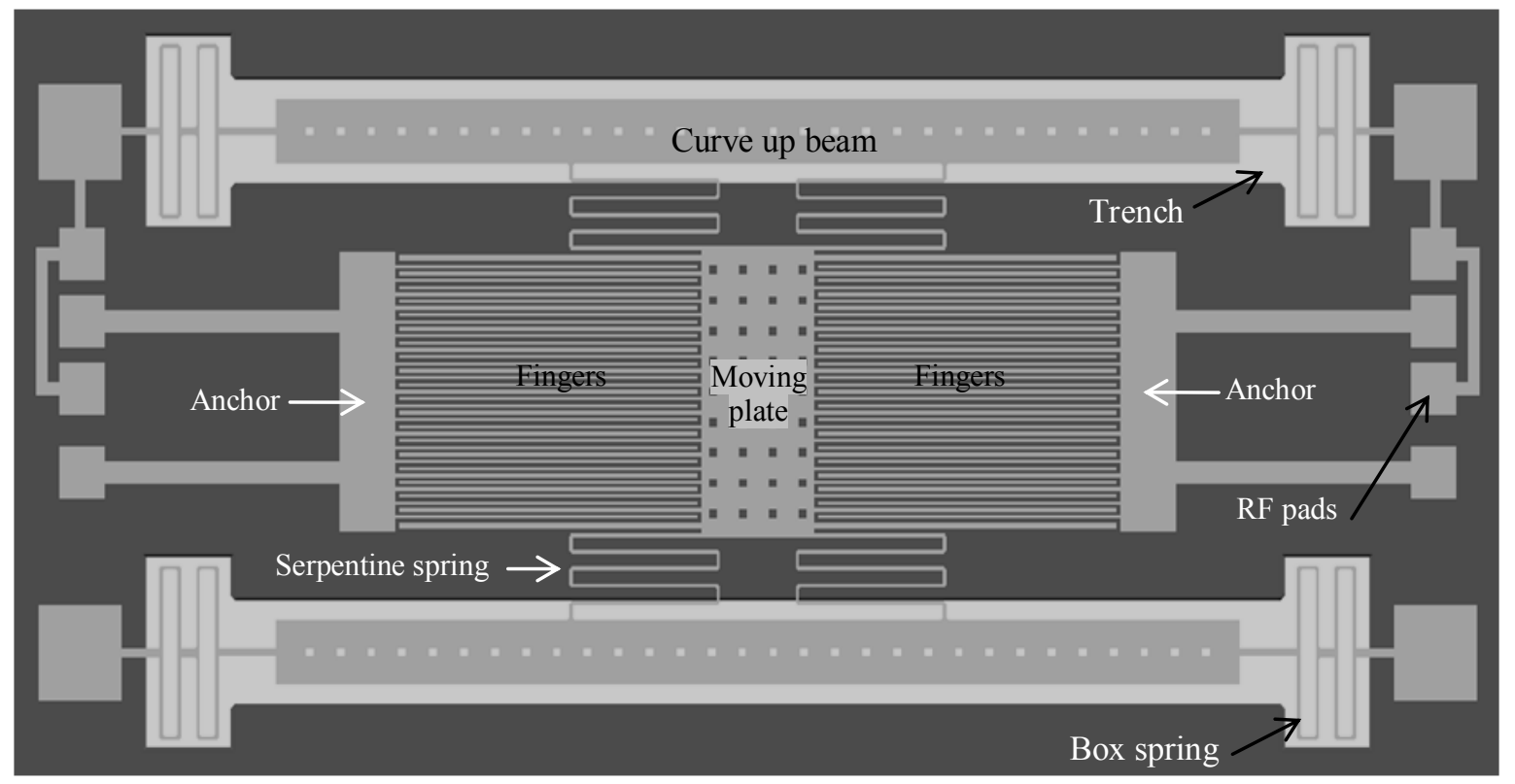

(b)

Figure 6-7 Capacitor with reduces pad, strips and anchor sizes (a) 3D view and (b) top view 
The parasitic capacitance calculated is assumed it be constant when the actuation voltages are varied. This capacitance now represents the parasitic capacitance due to pads, strips and anchors of the capacitor towards the substrate. The total pads strip and anchor areas in C7 design is $1.305 \mathrm{~mm}^{2}$ and produces $1.3778 \mathrm{pF}$ parasitic capacitance.

The final optimal design of the capacitor consists of 20 moving fingers on each side and minimum pads, strip and, anchors sizes of area $\sim 0.4855 \mathrm{~mm}^{2}$. The parasitic capacitance is proportional to the areas of these structures. Thus, the parasitic capacitance of optimized design with pad and strips is found to be $0.513 \mathrm{pF}$.

\subsection{Optimization of tuning ratio and quality factors by trench}

High dielectric constant substrates incorporate losses which tend to reduce the quality factors which are resulted from the high permittivity of the material. The dielectric constant of the silicon substrate in the MetalMUMPs process is 11.9. Large dielectric constant substrates produce high parasitic capacitance. Figure 6-8 shows the 3D view of the optimized capacitor with trenches.

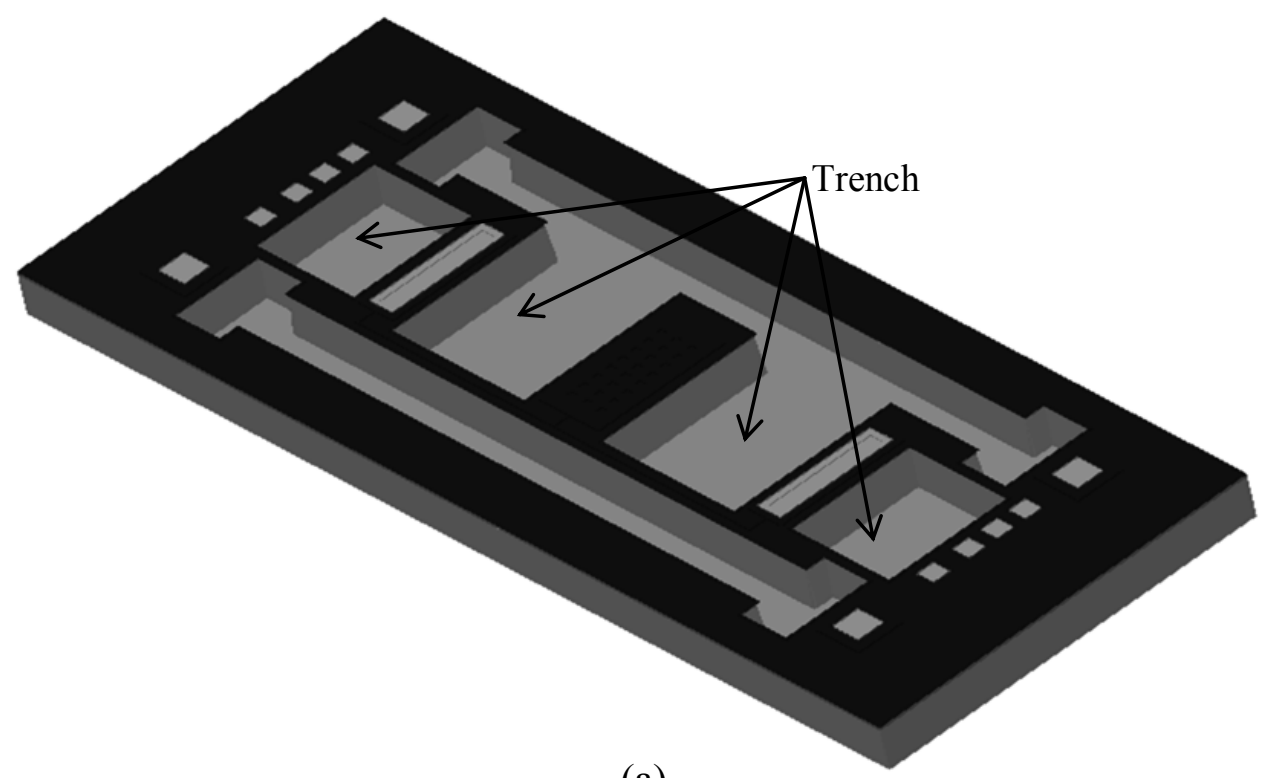

(a) 


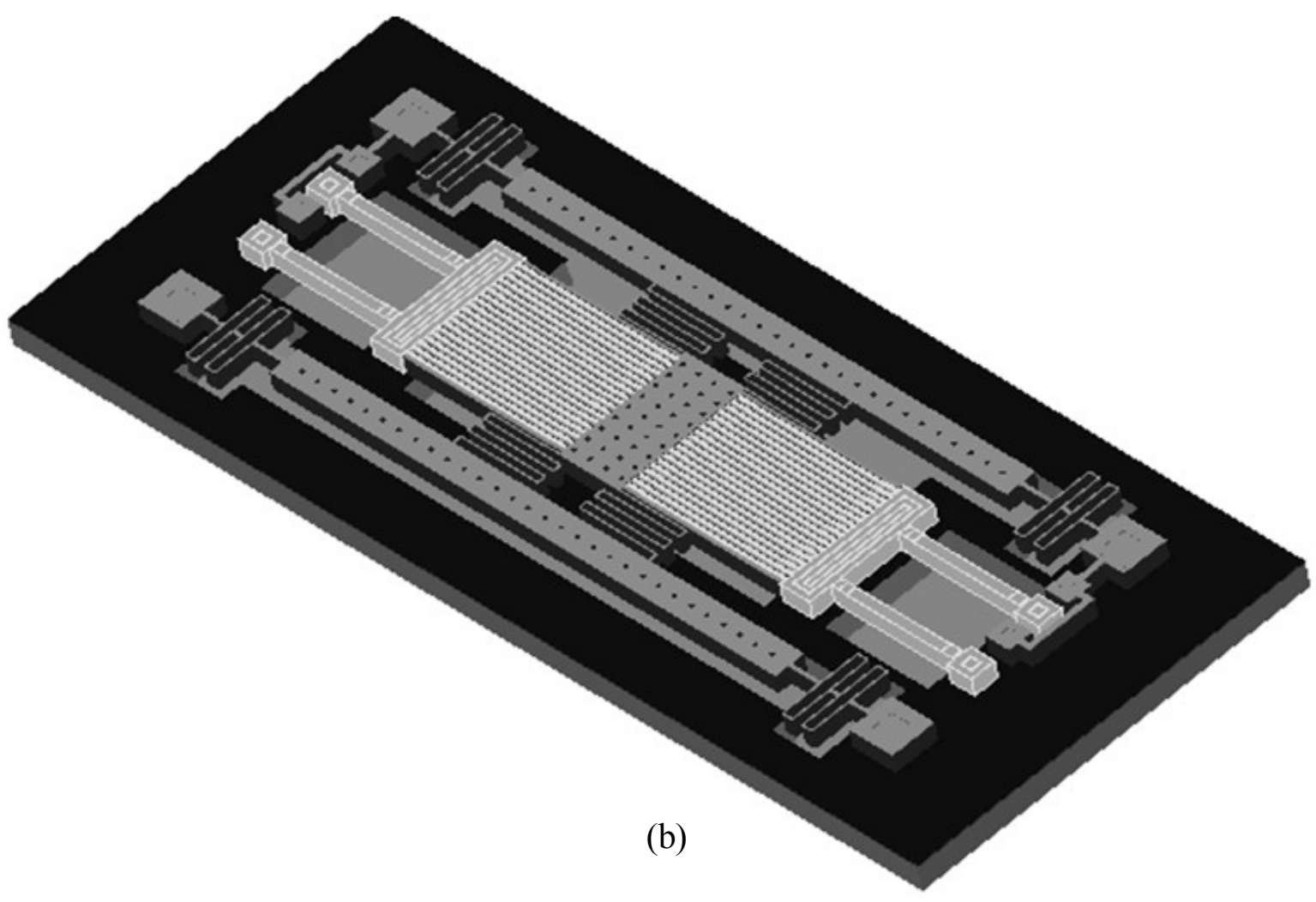

Figure 6-8 Optimized capacitor (a) trench and (b) 3D view with trench One important technique to reduce the parasitic capacitances is by lowering the dielectric constant. However, since the fabrication process used to develop the tunable capacitors is standard process it costly to replace the silicon with low dielectric substrate. However, the MetalMUMPs process allows a $25 \mu \mathrm{m}$ deep trench which can be created in the substrate by deep reactive ion $(\mathrm{KOH})$ etching. Using the trench an air dielectric of $25 \mu \mathrm{m}$ thickness can be formed between the fingers of the capacitor structures and the substrate. Two dielectric layer stacks are formed: the air $\left(\varepsilon_{r}=1.0\right)$ and the silicon $\left(\varepsilon_{r}=11.9\right)$ layers. It is known that the dielectric constant of a stack of can be computed by the equation:

$$
\varepsilon_{e q}=\left(\sum_{n=1}^{N} \frac{t_{n}}{\varepsilon_{n}}\right)^{-1}\left(\sum_{n=1}^{N} t_{n}\right)
$$

The total thickness of the layers is given by 


$$
t=\left(\sum_{n=1}^{N} t_{n}\right)
$$

From equation (6.14) the effective dielectric constant of the substrate with trench is lowered by cutting out the substrate. The effective dielectric constant after the trench is etched is 8.48 . The technique can reduce the parasitic capacitance.

Table 6-4 shows the tuning ratios and the quality factors of the optimized capacitor including the optimized finger dimensions and the reduced parasitic capacitance.

Table 6-4 Summary of tuning ratio based on optimized finger, strips, pads and anchor sizes

\begin{tabular}{cccc}
\hline Finger length $(\mu \mathrm{m})$ & $\begin{array}{c}\text { Capacitnace }(\mathrm{pF}) \text { at } 0 \mathrm{~V} \\
/ 162.5 \mathrm{~V}\end{array}$ & $\begin{array}{c}\text { Tuning ratio (\%) } \\
(1.26 / 2.45\end{array}$ & $\begin{array}{c}\text { Quality factor } \\
(0.8 \mathrm{GHz})\end{array}$ \\
\hline 800 & 194.4 & $131 / 54$ \\
\hline & Parasitic capacitance $=0.404 \mathrm{fF}$ \\
& Number of fingers $=20$ \\
\hline
\end{tabular}

\subsection{Advantages of novel tunable capacitor}

Optimization techniques have been investigated to obtain high tuning ration and quality factors using parameters such as large number of fingers, longer fingers, reduced anchor, strip length and pad sizes. It proved that these factors extremely influence the tuning ratio and the quality factors of the capacitor. Based on the combined experimental analysis and simulations, the tunable capacitor has the following advantages:

\subsubsection{High tuning ratio}

Tuning ratio of $153.5 \%$ can be achieved by the optimization of the fingers. The addition fingers of $800 \mu \mathrm{m}$ increased the total capacitance at $0 \mathrm{~V}$. When fingers of the capacitor move down the change in the capacitors are the capacitance change $\Delta \mathrm{C} / \mu \mathrm{m}$ is high for the optimized design compared with the original C7. As a result higher tuning ratio in terms finger dimensions were obtained. The reduced gap between the fixed fingers and the moving fingers is narrows the gap of the fringing fields and results high capacitance density. 
Pad and strip sizes minimization further enhanced the tuning ratio by reducing the parasitic capacitance that propagates from the substrate. The final tuning ratio which optimized in terms of finger dimensions, pads and strip sizes reached 194.4\%.

\subsubsection{High quality factors}

The second advantages of the tunable capacitors developed in this thesis work have high quality factors. The quality factors obtained in this thesis work range from 38-163 for C7 design. However, for the optimized design due to the increased number and length of the capacitor fingers the value of the quality factor obtained is low compared with the experimentally obtained $\mathrm{Q}$ values. For the optimized design quality factor of 59 was determined when the moving fingers are at the down state at $0.8 \mathrm{GHz}$. The $\mathrm{Q}$ value of 131 was calculated at $0.8 \mathrm{GHz}$ when the fingers are at the up state.

\subsubsection{High linearity}

The capacitance-voltage relations in the novel comb drive actuator show high linearity. Therefore, the capacitors here provide high linearity with the actuation voltage.

\subsubsection{High power handling}

High actuation voltages up to $200 \mathrm{~V}$ were required to drive the tunable capacitors. This indicates that the tunable capacitors are capable of handling high RF power signal. The capacitors developed here can support over $50 \mathrm{~V}$ equivalent RF voltage.

\subsubsection{Other advantages}

The fourth advantage of the tunable capacitor in this thesis work is no dielectric charging which is the result of the air gap between the fingers of the capacitor. Parallel plate switchable tunable capacitors have thin dielectric films [116] which are commonly used to prevent the contact between the moving plate and the fixed plates. The dielectric films are likely store charges and break due to the heating effect of RF power. This effect causes arcs and short circuits between the capacitor plates. All the capacitors presented in this thesis work are free from dielectric charging effects.

Pull-in instability is completely eliminated in the capacitors presented in this work. The electrostatic force between the sidewalls of the moving fingers and the fixed fingers are balanced 
by the neighboring fingers. As a result when actuation voltages are applied across the fingers, the moving fingers move linearly down and the gap between the fingers does not change.

\subsubsection{Comb drive actuators}

This section makes comparison of the novel vertical comb-drive capacitor with the existing comb actuators. Comb drive based capacitors have been developed in [117-132]. The arrangement of the comb drives are either lateral or vertical [117, 119]. Tang et al used [119] used levitation force perpendicular to the substrate to drive the vertical comb of a resonator. Additional ground plane was used to protect the moving from sticking to the substrate fabricated using Polysilicon surface micromachining process. The displacement measured at $30 \mathrm{~V}$ was found to be $2 \mu \mathrm{m}$ with 18 moving fingers and 19 fixed fingers. Lateral comb drives [118-120] have side pull-in instability which determined by the design of the spring combs [120-123]. Thick structural layer fabrication processes were used to fabricate lateral combs as in [122] and required deep reactive silicon etching (Bosch) process that makes the fabrication costly [123]. Patterson et al [128] developed angular vertical comb drive for which achieved large angular deflection $\geq 10$ degree at $100 \mathrm{~V}$. The same design principle used in [128] has been applied to develop tunable capacitor in [129] fabricated on glass substrate followed by flipping an SOI layer of $25 \mu \mathrm{m}$ silicon device layer bonding. A Benzocyclobuten (BCB), polymer, hinges were used to connect two sets of comb fingers and to create angular misalignment after the glass substrate is etched away. High quality factor of 273 at $1 \mathrm{GHz}$ was obtained with tuning ratio of 33:1 and 40V driving voltage. The materials used in the fabrication have low dielectric constants and dielectric losses. However, this design requires high fabrication cost. The BCB used has also limited thermal stability and the fabrication process used bulk micromachining of substrate. The fabrication cost of vertical comb drive structures is very expensive [130] and need complicated process steps. The first known method of fabricating staggered vertical combs is using surface and bulk micromachining processes. Trench cutouts and trench refilling of Polysilicon were needed to develop vertical micro-actuators in [131] which made design complexity. The second method involves bonding process on two SOI layer and repeated deep reactive ion etch (DRIE) [132]. In such designing process of vertical comb drives misalignment is a big challenge [130]. This necessitates self aligning methods for accurate alignment.

The novel comb-drive actuators developed in this thesis work has the following key advantages: 
(1) Only single layer was used to create two layers of the comb drive actuator

(2) Better lateral finger stability because of the balanced electrostatic force on the side walls of the moving fingers and the fixed fingers

(3) Better thermal stability since the nickel metal has lower thermal conductivity and lower thermal expansion coefficient

(4) There is no mask misalignment in the fabrication process, as a result the gap between the moving fingers and the fixed fingers remains constant after fabrication

(5) High quality factor tunable capacitors were designed due to the thickness of the metal

(6) Fabricated using commercially available and relatively cheap surface micro fabrication process

\subsubsection{Drawbacks}

The tunable capacitors however have the following drawbacks:

(1) High driving voltage is required $(<200 \mathrm{~V})$

(2) High RF signal loss due to the thickness of the silicon $(675 \mu \mathrm{m})$ and high dielectric constant (11.9). Low dielectric constant substrates provide low loss (e.g. glass)

(3) High parasitic capacitance

\subsection{Chapter summary}

This chapter entirely focused on increasing the tuning ratio of the comb drive tunable capacitor developed in Chapter 5. Two techniques were proposed to increase the tuning ratio of the capacitors. The first technique used finger dimensions and gap between the moving and the fixed fingers. The width of the outer moving fingers is taken an important parameter to avoid the side instability caused by the one-sided electrostatic force as the actuation voltage increases. It was found that thicker outer fingers resist the growing electrostatic force at higher voltages. Reduction of the area occupied by the pads, anchors and strips was the second technique used to minimize the parasitic capacitance and as a result to increase the tuning ratio. The combination of the above techniques raised the tuning ratio to $194.4 \%$ with a quality factor of 131 at $0.8 \mathrm{GHz}$. Cutting a trench in the silicon substrate is suggested as an additional method to reduce the parasitic capacitances and RF power losses. 


\section{Conclusions and future work}

This thesis work presented the first attempt of implementation of MEMS tunable capacitors driven by repulsive-force electrostatic actuators. The thesis developed a novel residual stress gradient based vertical comb-drive actuator using a single nickel layer process (MetalMUMPs) and the tunable capacitors made of it.

\subsection{Contributions}

This thesis has come across the design of one actuator and two novel tunable capacitors. The thesis work has added two major results summarized below.

\subsubsection{Electrostatic repulsive- force actuated tunable capacitor}

The tunable capacitor used repulsive-force actuators to drive the moving plate out of plane and away from the fixed plate. The gap between the plates of the capacitor increases with the driving voltage. The upward motion of the moving plate results in high linearity between the actuation voltage and the capacitance. Therefore high linearity is one advantage of the repulsive-force actuator driven tunable capacitors. Another advantage of the tunable capacitor is the high tuning ratio. A tuning ratio of 2.62 has been obtained at $160 \mathrm{~V}$ actuation voltage. This design does not suffer from the pull-in instability caused by the RF signal and the actuator driving voltage in comparison to other MEMS tunable capacitors. Dimples have been used on the moving plates to prevent short circuiting between the fixed and the moving plates of the capacitor by enabling the dimples of the moving plate to land on the substrate via holes in the fixed plate in case of excessive RF power.

\subsubsection{Novel residual stress gradient based vertical comb-drive actuator and its application to tunable capacitor}

MetalMUMPs is a matured and commercially available microfabrication process which can lead to low cost and high yield mass production of MEMS devices. The MetalMUMPs provides one thick metal layer (20 $\mu \mathrm{m}$ nickel) which can result in low RF power loss to fabricate high quality factor tunable RF MEMS components.. However conventional vertical comb actuators required two layers to construct the fixed and moving fingers. This thesis developed a novel method to use the one nickel layer process (MetalMUMPs) to fabricate two-layer vertical comb-drive actuators to make tunable RF components by utilizing the residual stress gradient associated with 
the process. This novel actuator residual stress gradient based vertical comb actuator uses two curve-up beams to raise the moving fingers vertically above the fixed fingers. When the voltage is applied the moving fingers move down and towards the fixed fingers relative to the curve-up beams which does not move after raising the moving fingers during releasing stage of the fabrication. The novel residual stress gradient based vertical comb actuator has been theoretically and experimentally verified. Tunable capacitors based on the novel actuator have been successfully developed, which are able to tune the capacitance between fixed and moving fingers and achieved high quality factor, high linearity, high tuning ratio and high RF power handling capability. In addition, the tunable capacitor does not suffer from the misalignment between two layers since it is fabricated from the single nickel layer. The thesis provided an effective solution to solve the side pull-in effect of the tunable capacitor by changing the thickness of most outside fingers.

The significance the residual stress gradient actuators is that there is no requirement of bonding and no misalignment error between the moving fingers and the fixed fingers. Because of the absence of the misalignment error, there will be less possibility for the formation unbalanced electrostatic forces on the fingers of the actuators. Therefore, the fingers of the capacitor are stable at any actuation voltage. This implies to the conclusion that the stiffness of the springs remains constant or linear.

\subsection{Future works and recommendations}

Further work can be continued on the work presented in this thesis. The future possible improvements and additional research works are summarized in this section.

\subsubsection{Tunable capacitors driven by the repulsive-force actuator}

The work on the tunable capacitor can be extended further to develop devices which have high performance. It can be fabricated on a low resistive dielectric substrate such as glass. Silicon substrate and silicon nitride usually store charges and cause induce electric field that pulls suspended layers and results in stiction problem.

The stiction problem found in this thesis work could be eliminated minimized by using grounding loops to suppress the coupling electric fields from the neighboring electrodes. The total field inside the loop remains only the field of the RF electrode. Therefore, there will not be 
induced field from the outside fingers. Another method to suppress the fields coming from the DC fingers is to use shielding strip that encloses the unaligned fixed fingers of the repulsive actuator

The electrostatic fields that propagate through the nitride can be suppressed by covering the nitride itself with a large ground electrode.

\subsubsection{Tunable capacitors driven by the novel vertical comb actuator}

The parasitic capacitance of this tunable capacitor arises mainly from the substrate and the nitride layers. The parasitic capacitance loss is extremely high for silicon substrates and nitride layers. Low dielectric constant substrates such as glass and sapphire have low dielectric constants. They can be used to replace the silicon substrate so that capacitance losses are reduced.

Electromagnetic actuation is suggested to replace the electrostatic actuation that demands high voltage. Even though electromagnetic actuators require high power and complex design, the force generated to drive the fingers is high compared with electrostatic forces.

In Chapter 5 experimental investigation of the power handling was analyzed using equations based on the actuation voltages. Low power RF signal was used in the experiment. In the future high RF power levels need to be applied to test the behavior of the tunable capacitor when the high RF power is superimposed on the DC actuation voltage.

The RF power generates heating due to resistance of the nickel. This effect needs to be investigated in the future.

Substrate trenches were used in the thesis to reduce the parasitic capacitances. Designs with trenches are compared and the significance of trench to reduce the parasitic capacitance is mentioned. Experimental tests on this are recommended in the future. 


\section{Appendices}

\section{Appendix A Equations of curvature of fitted curve-up beams-Matlab}

\section{Beam B1}

$\mathrm{y}=\mathrm{p} 1 * \mathrm{z}^{\wedge} 6+\mathrm{p} 2 * \mathrm{z}^{\wedge} 5+\mathrm{p} 3 * \mathrm{z}^{\wedge} 4+\mathrm{p} 4 * \mathrm{z}^{\wedge} 3+$ $\mathrm{p} 5^{*} \mathrm{z}^{\wedge} 2+\mathrm{p} 6^{*} \mathrm{z}+\mathrm{p} 7$

where $\mathrm{z}$ is centered and scaled:

$\mathrm{z}=(\mathrm{x}-\mathrm{mu}) / \operatorname{sigma} \mathrm{mu}=1.3741 \mathrm{e}+05$

sigma $=714.17$

Coefficients:

$\mathrm{p} 1=0.27875 \mathrm{p} 2=0.015256 \mathrm{p} 3=-0.79998$

$\mathrm{p} 4=-0.033069 \mathrm{p} 5=-2.2842 \mathrm{p} 6=-0.02796$

p7 $=6.4492$

Norm of residuals $=1.5338$

\section{Beam B2}

$\mathrm{y}=\mathrm{p} 1 * \mathrm{z}^{\wedge} 6+\mathrm{p} 2 * \mathrm{z}^{\wedge} 5+\mathrm{p} 3 * \mathrm{z}^{\wedge} 4+\mathrm{p} 4 * \mathrm{z}^{\wedge} 3+$ $\mathrm{p} 5 * \mathrm{z}^{\wedge} 2+\mathrm{p} 66^{*} \mathrm{z}+\mathrm{p} 7$

where $\mathrm{z}$ is centered and scaled:

$\mathrm{z}=(\mathrm{x}-\mathrm{mu}) / \operatorname{sigma} \mathrm{mu}=1.4134 \mathrm{e}+05$

sigma $=721.72$

Coefficients:

$\mathrm{p} 1=0.28773 \mathrm{p} 2=0.023832 \mathrm{p} 3=-0.82175$

$\mathrm{p} 4=-0.046093 \mathrm{p} 5=-2.3646 \mathrm{p} 6=-0.060894$

p7 $=6.6945$

Norm of residuals $=1.4855$

\section{Beam B3}

$\mathrm{y}=\mathrm{p} 1^{*} \mathrm{z}^{\wedge} 6+\mathrm{p} 2 * \mathrm{z}^{\wedge} 5+\mathrm{p} 3 * \mathrm{z}^{\wedge} 4+\mathrm{p} 4{ }^{*} \mathrm{z}^{\wedge} 3+$ $\mathrm{p} 5^{*} \mathrm{z}^{\wedge} 2+\mathrm{p} 6^{*} \mathrm{z}+\mathrm{p} 7$

where $\mathrm{z}$ is centered and scaled:

$\mathrm{z}=(\mathrm{x}-\mathrm{mu}) / \operatorname{sigma} \mathrm{mu}=1.4553 \mathrm{e}+05$

sigma $=725.39$

Coefficients:

$\mathrm{p} 1=0.45732 \mathrm{p} 2=0.089417 \mathrm{p} 3=-1.3466$

$\mathrm{p} 4=-0.17816 \mathrm{p} 5=-3.8517 \mathrm{p} 6=-0.24943$

p7 $=11.207$

Norm of residuals $=3.3448$

\section{Beam B4}

$\mathrm{y}=\mathrm{p} 1 * \mathrm{z}^{\wedge} 6+\mathrm{p} 2 * \mathrm{z}^{\wedge} 5+\mathrm{p} 3 * \mathrm{z}^{\wedge} 4+\mathrm{p} 4 * \mathrm{z}^{\wedge} 3+$ $\mathrm{p} 5^{*} \mathrm{z}^{\wedge} 2+\mathrm{p} 6^{*} \mathrm{z}+\mathrm{p} 7$

where $\mathrm{z}$ is centered and scaled:

$\mathrm{z}=(\mathrm{x}-\mathrm{mu}) / \operatorname{sigma} \mathrm{mu}=1.5054 \mathrm{e}+05$

sigma $=745.73$
Coefficients:

$\mathrm{p} 1=0.31922 \mathrm{p} 2=0.04668 \mathrm{p} 3=-0.59915$

$\mathrm{p} 4=-0.01509 \mathrm{p} 5=-3.4045 \mathrm{p} 6=-0.31075$

p7 $=7.2312$

Norm of residuals $=1.7121$

\section{Beam B5}

$\mathrm{y}=\mathrm{p} 1^{*} \mathrm{z}^{\wedge} 6+\mathrm{p} 2 * \mathrm{z}^{\wedge} 5+\mathrm{p} 3 * \mathrm{z}^{\wedge} 4+\mathrm{p} 4 * \mathrm{z}^{\wedge} 3+$ $\mathrm{p} 5 * \mathrm{z}^{\wedge} 2+\mathrm{p} 6 * \mathrm{z}+\mathrm{p} 7$

where $\mathrm{z}$ is centered and scaled:

$\mathrm{z}=(\mathrm{x}-\mathrm{mu}) /$ sigma $\mathrm{mu}=1.5628 \mathrm{e}+05$

sigma $=739.5$

Coefficients:

$\mathrm{p} 1=0.23431 \mathrm{p} 2=0.031025 \mathrm{p} 3=-0.17143$

$\mathrm{p} 4=0.098183 \mathrm{p} 5=-3.6478 \mathrm{p} 6=-0.49255$

p7 $=6.4485$

Norm of residuals $=2.322$

\section{Beam B6}

$\mathrm{y}=\mathrm{p} 1{ }^{*} \mathrm{z}^{\wedge} 6+\mathrm{p} 2 * \mathrm{z}^{\wedge} 5+\mathrm{p} 3 * \mathrm{z}^{\wedge} 4+\mathrm{p} 4 * \mathrm{z}^{\wedge} 3+$ $\mathrm{p} 5{ }^{*} \mathrm{z}^{\wedge} 2+\mathrm{p} 6^{*} \mathrm{z}+\mathrm{p} 7$

where $\mathrm{z}$ is centered and scaled:

$\mathrm{z}=(\mathrm{x}-\mathrm{mu}) / \operatorname{sigma} \quad \mathrm{mu}=1.2897 \mathrm{e}+05$

sigma $=939.26$

Coefficients:

$\mathrm{p} 1=0.35738 \mathrm{p} 2=0.11978 \mathrm{p} 3=-0.83924$

$\mathrm{p} 4=-0.13989 \mathrm{p} 5=-2.9755 \mathrm{p} 6=-0.50366$

p7 $=7.0366$

Norm of residuals $=2.4639$

\section{Beam B7}

$\mathrm{y}=\mathrm{p} 1 * \mathrm{z}^{\wedge} 6+\mathrm{p} 2 * \mathrm{z}^{\wedge} 5+\mathrm{p} 3 * \mathrm{z}^{\wedge} 4+\mathrm{p} 4 * \mathrm{z}^{\wedge} 3$

$+\mathrm{p} 5 * \mathrm{z}^{\wedge} 2+\mathrm{p} 6^{*} \mathrm{z}+\mathrm{p} 7$

where $\mathrm{z}$ is centered and scaled:

$\mathrm{z}=(\mathrm{x}-\mathrm{mu}) /$ sigma $\mathrm{mu}=1.289 \mathrm{e}+05$

sigma $=984.92$

Coefficients:

$\mathrm{p} 1=0.86954 \mathrm{p} 2=-0.00040367 \mathrm{p} 3=-$

$1.73 \mathrm{p} 4=9.3085 \mathrm{e}-05 \mathrm{p} 5=-7.6902$

p6 $=0.0027963$ p $7=15.906$

Norm of residuals $=7.7608$ 
Appendix B Deformations of curve up beams C2, C3, C4, C6, C8, C9, C10
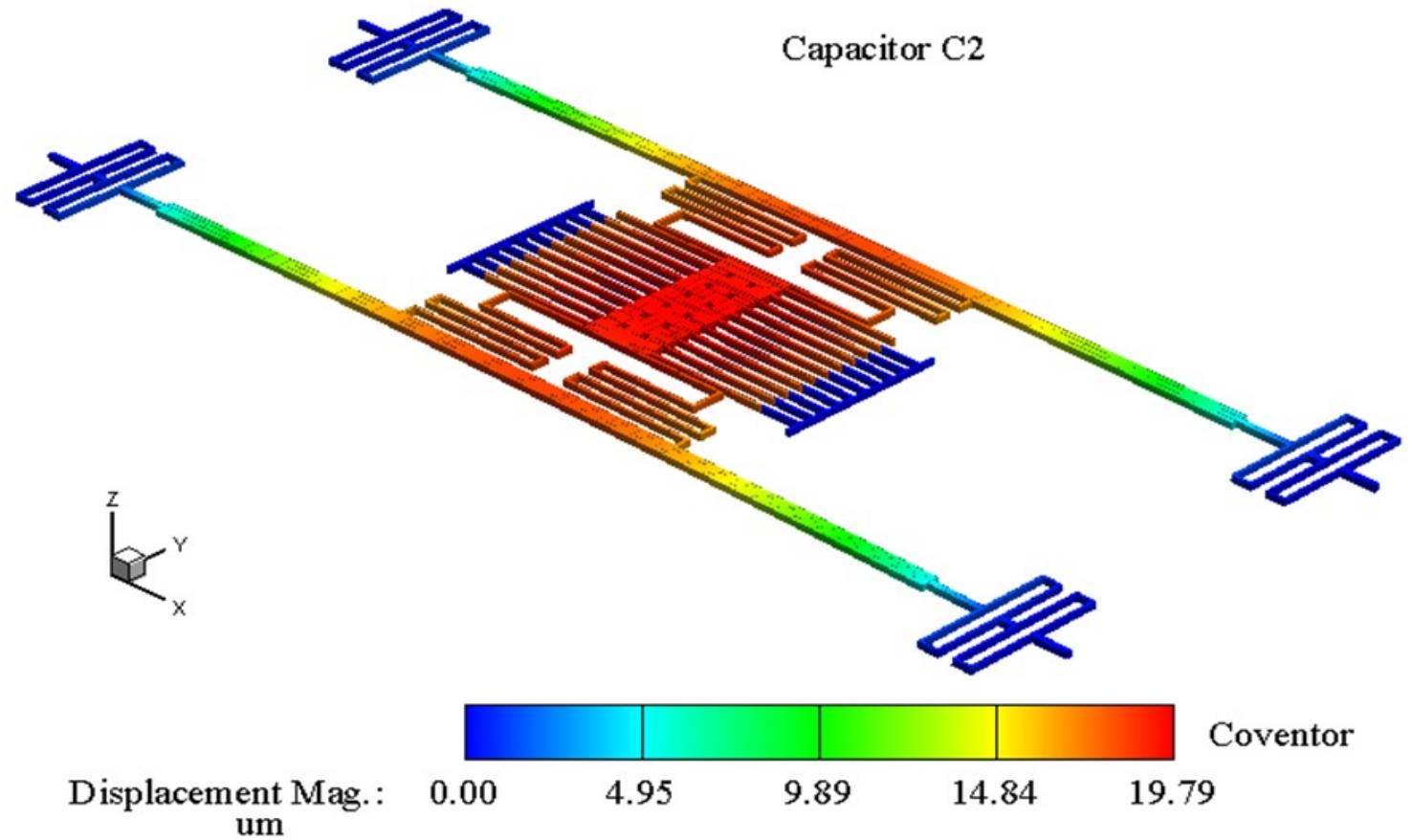

(a)
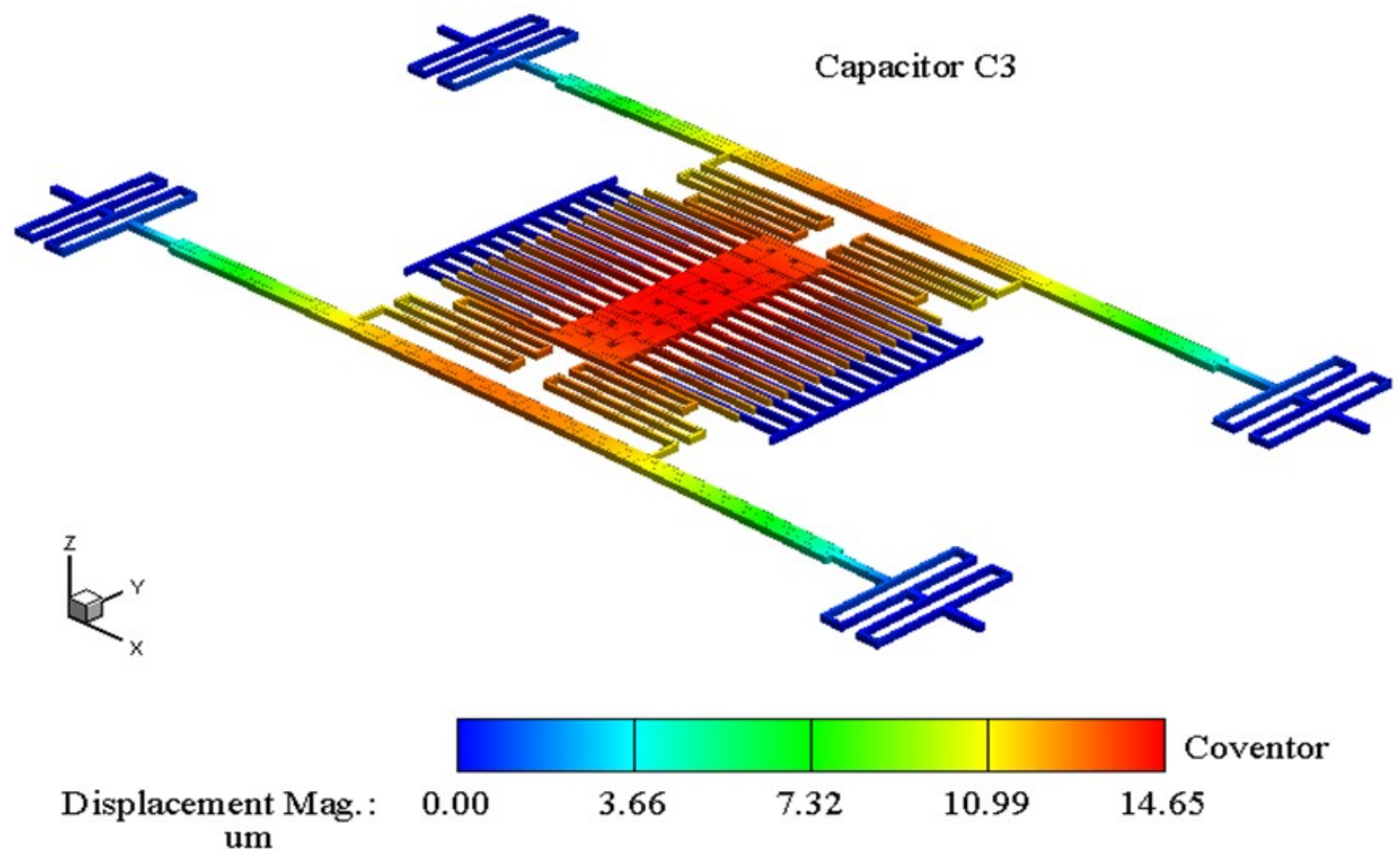

(b) 

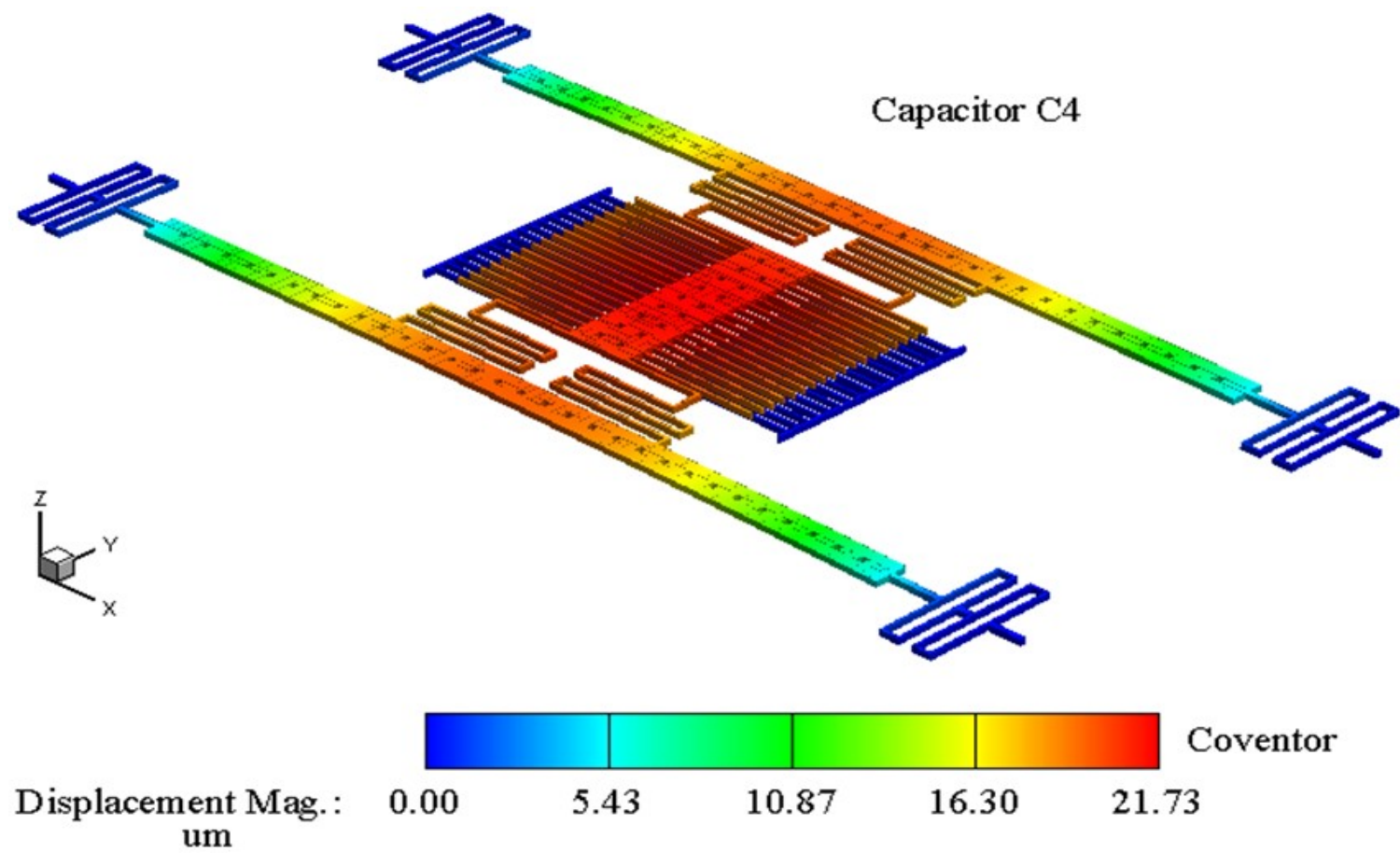

(c)
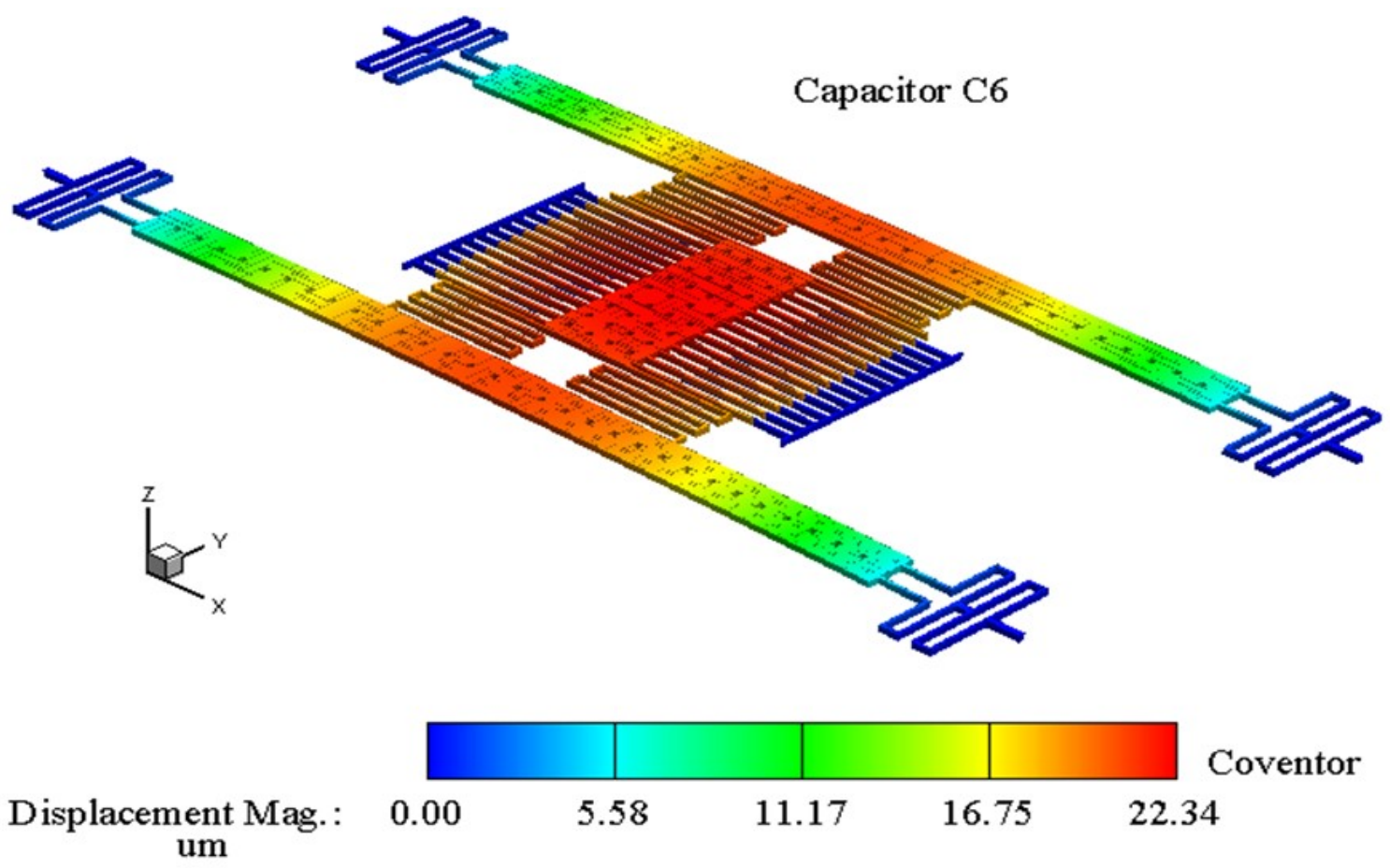

(d) 

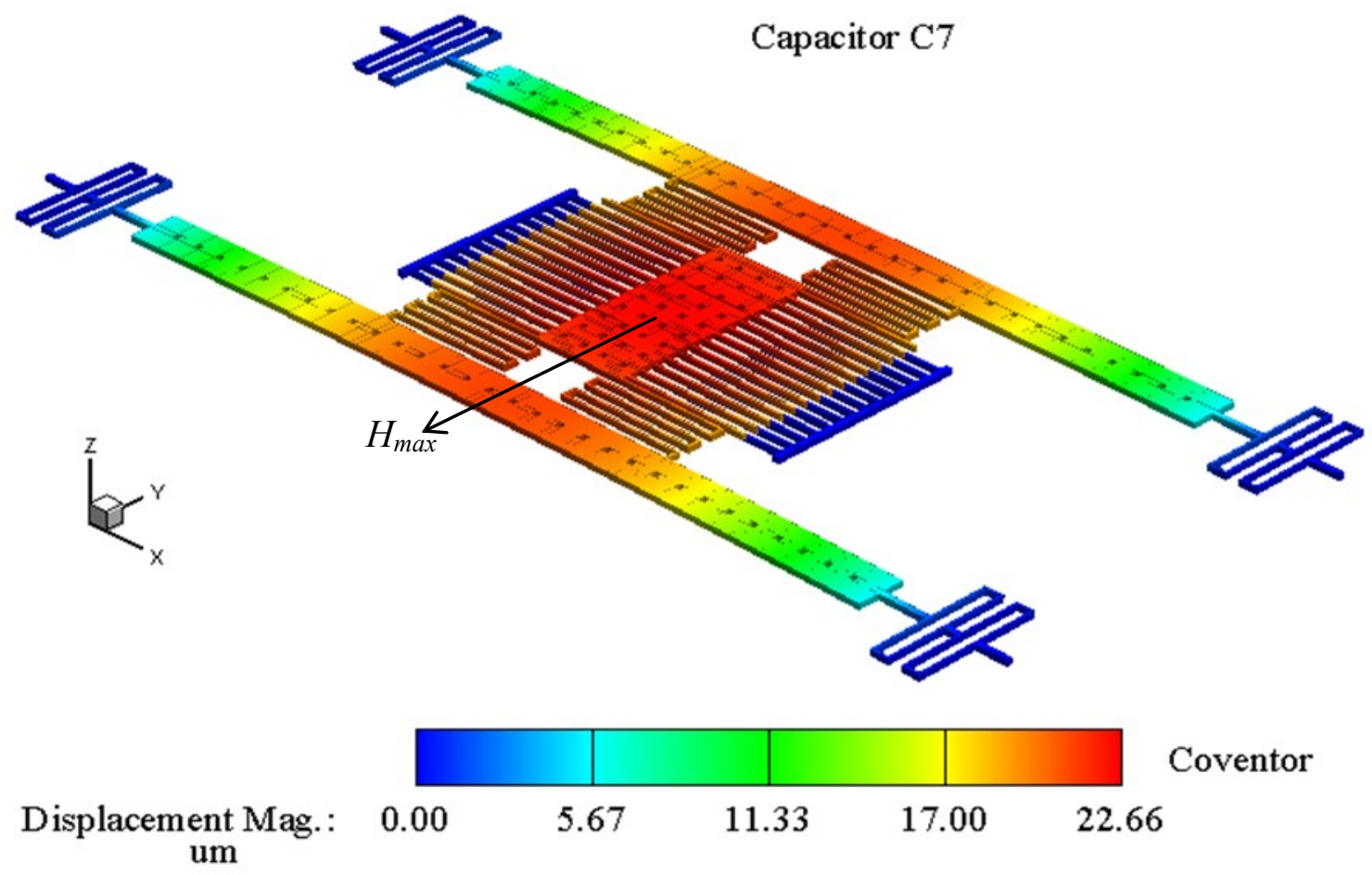

(e)
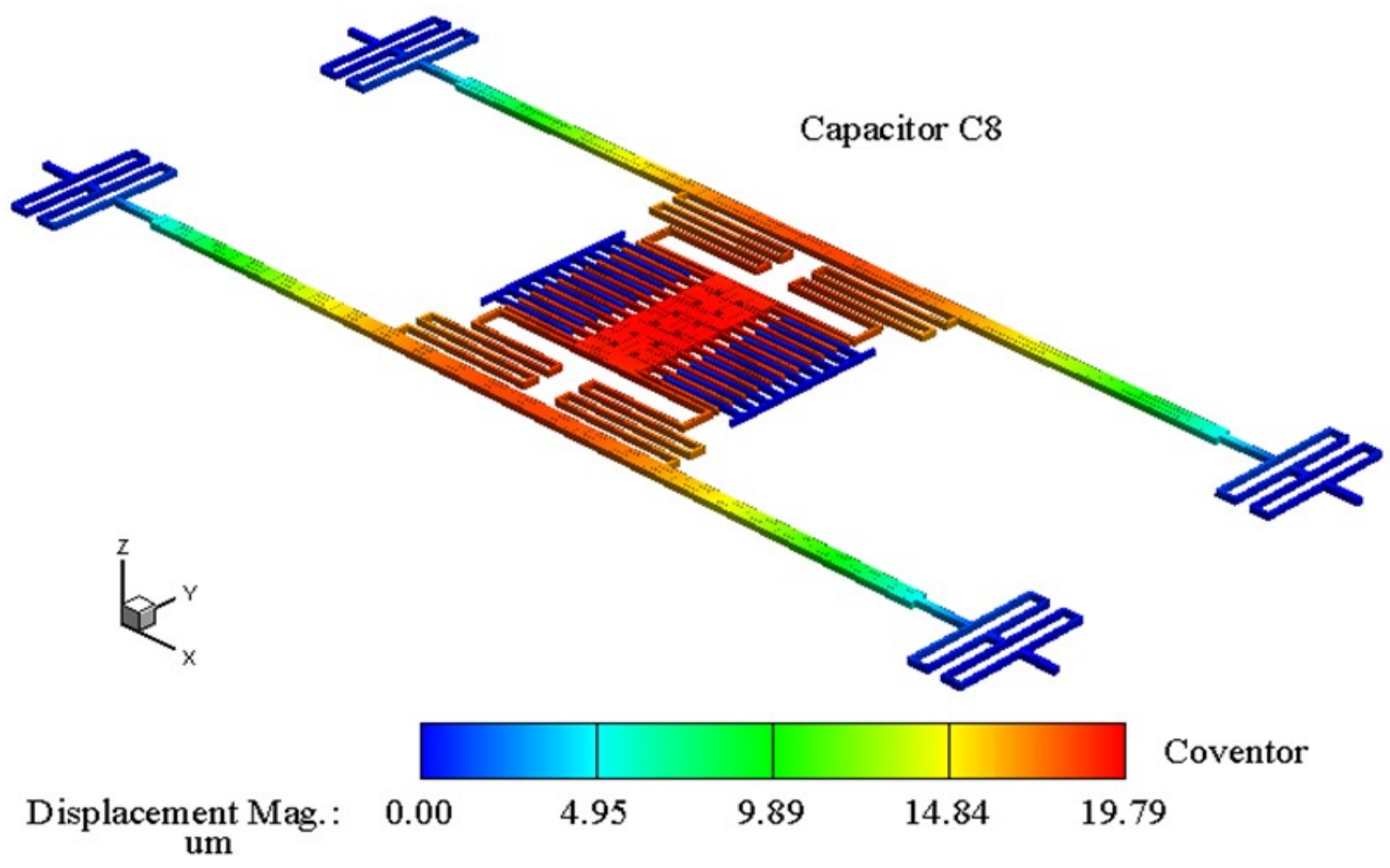

(f) 

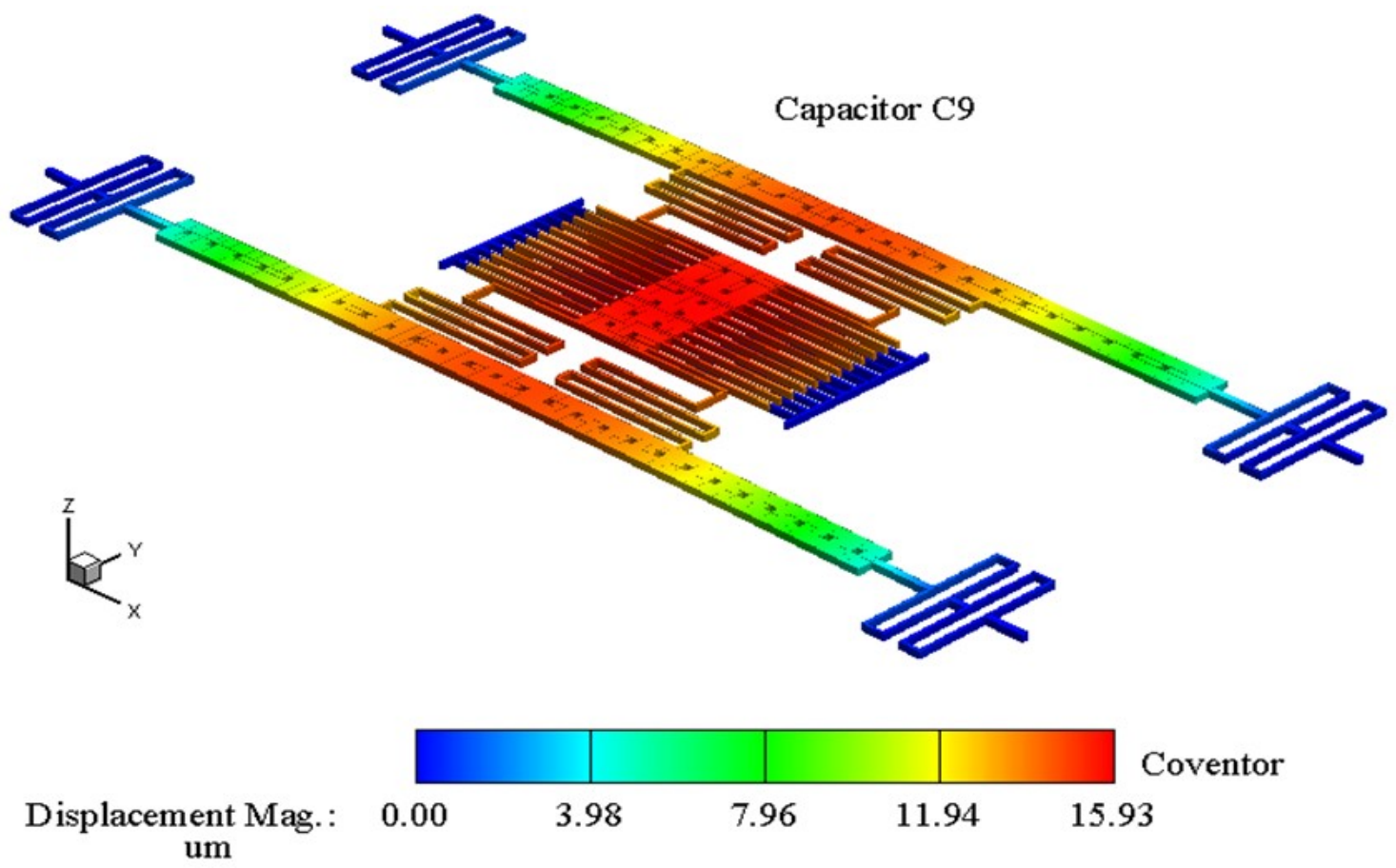

(g)
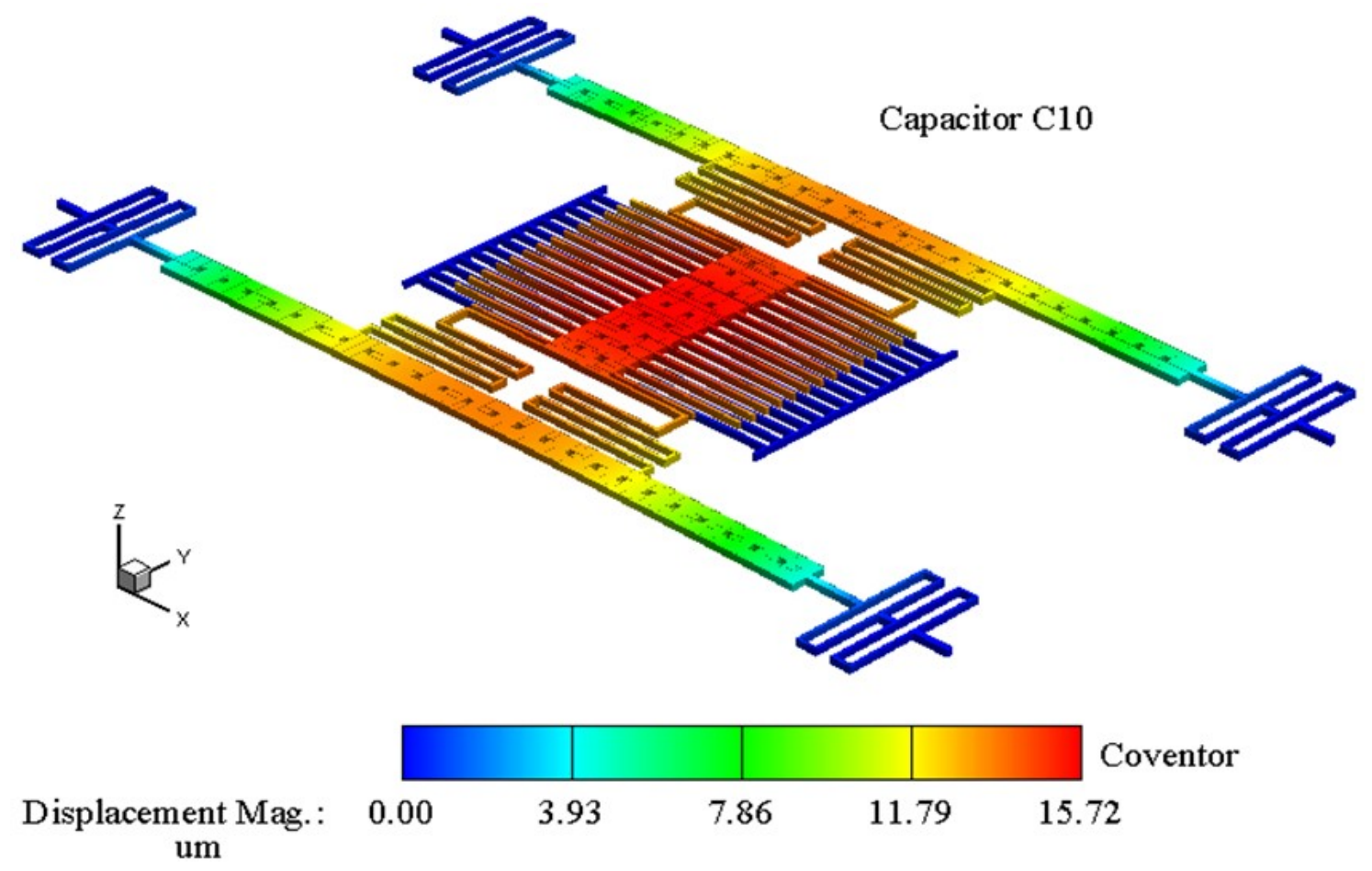

(h)

Figure B 1 Deformations of capacitors C1-C10 (a)-(j) 


\section{Appendix C NO voltage measurements (Chapter 5)}
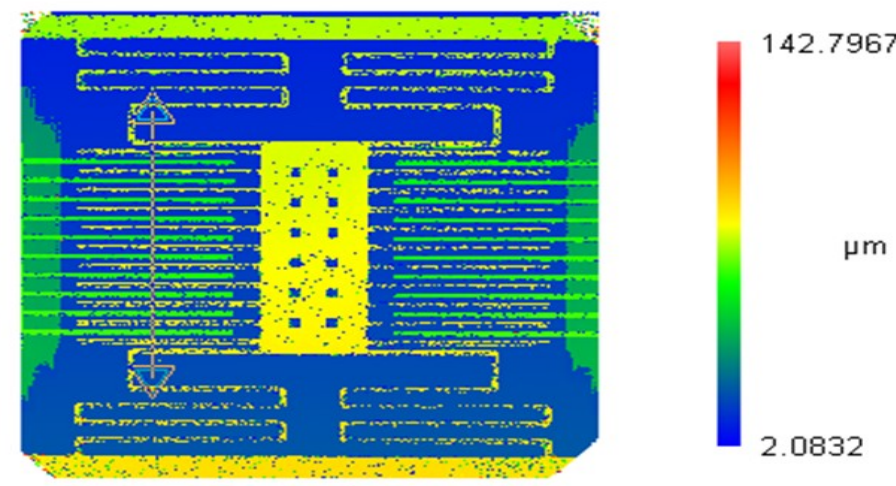

(a)

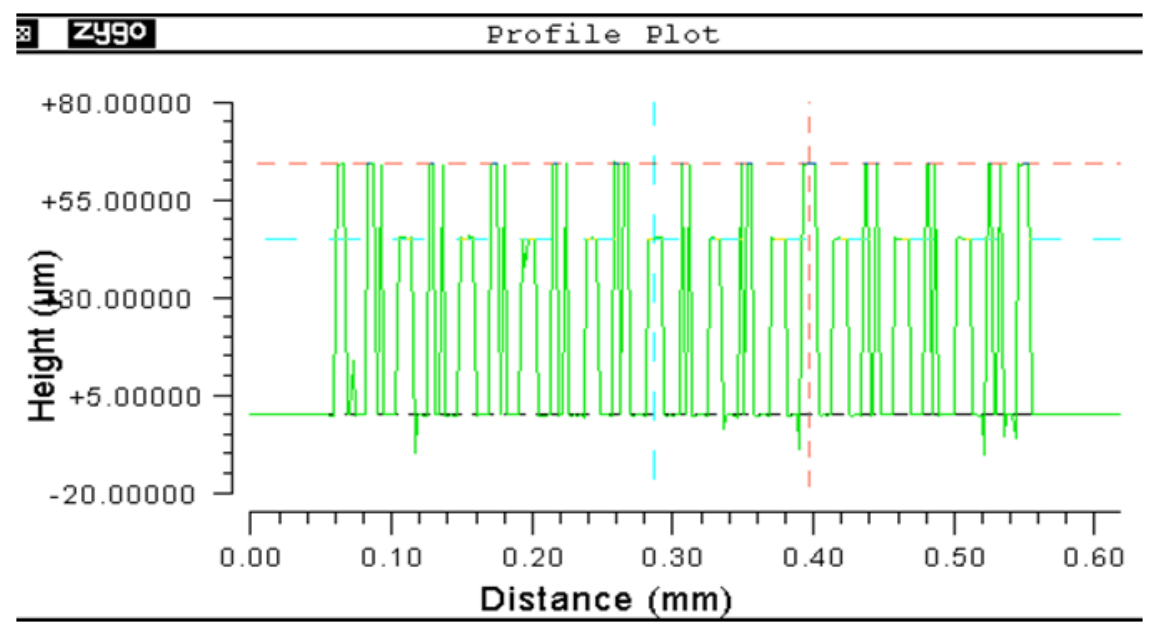

(b)
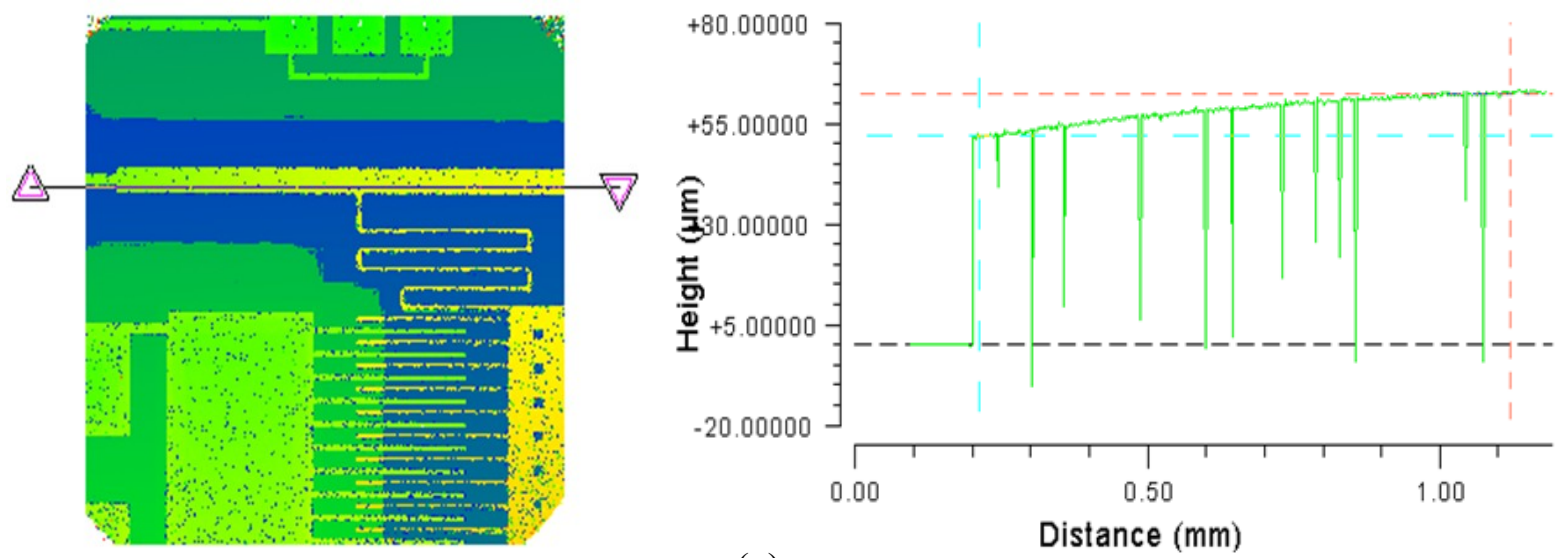

(c)

Figure C 1 Capacitor C2 (a) surface map and (b) profile plot (c) curve-up beam plot 


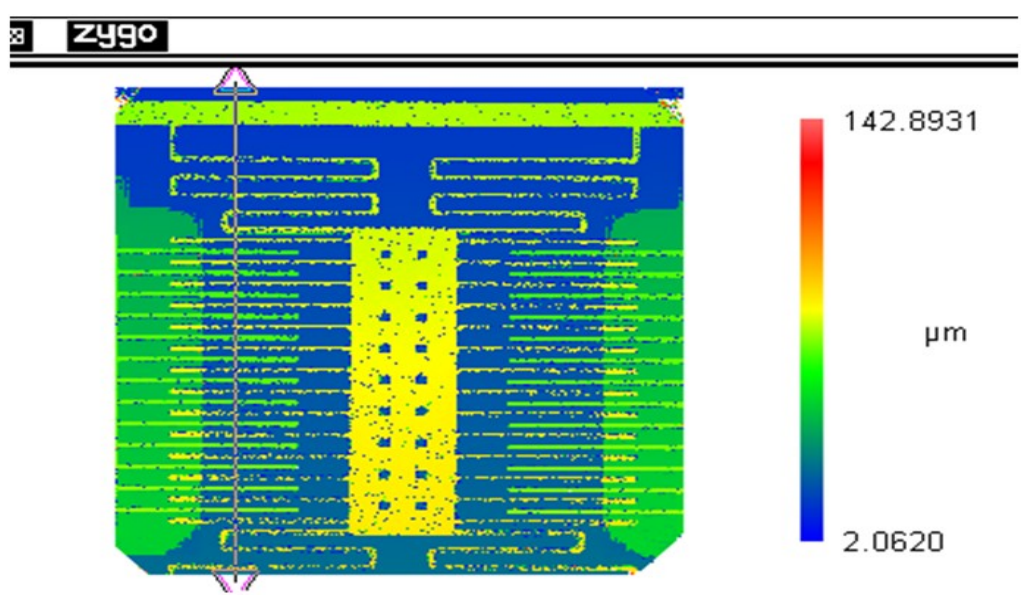

(a)

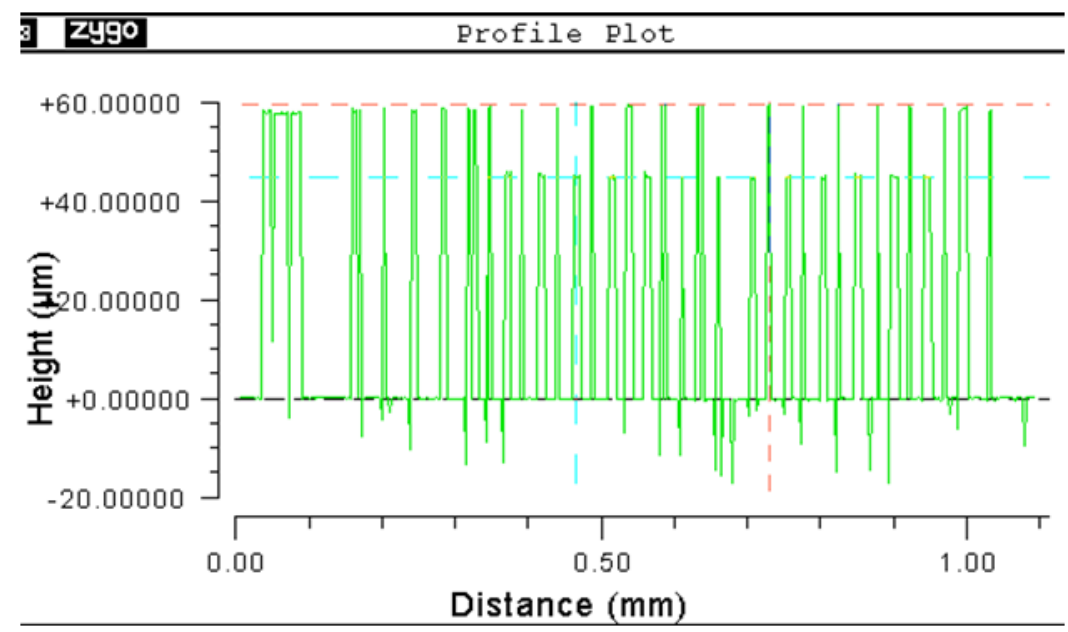

(b)
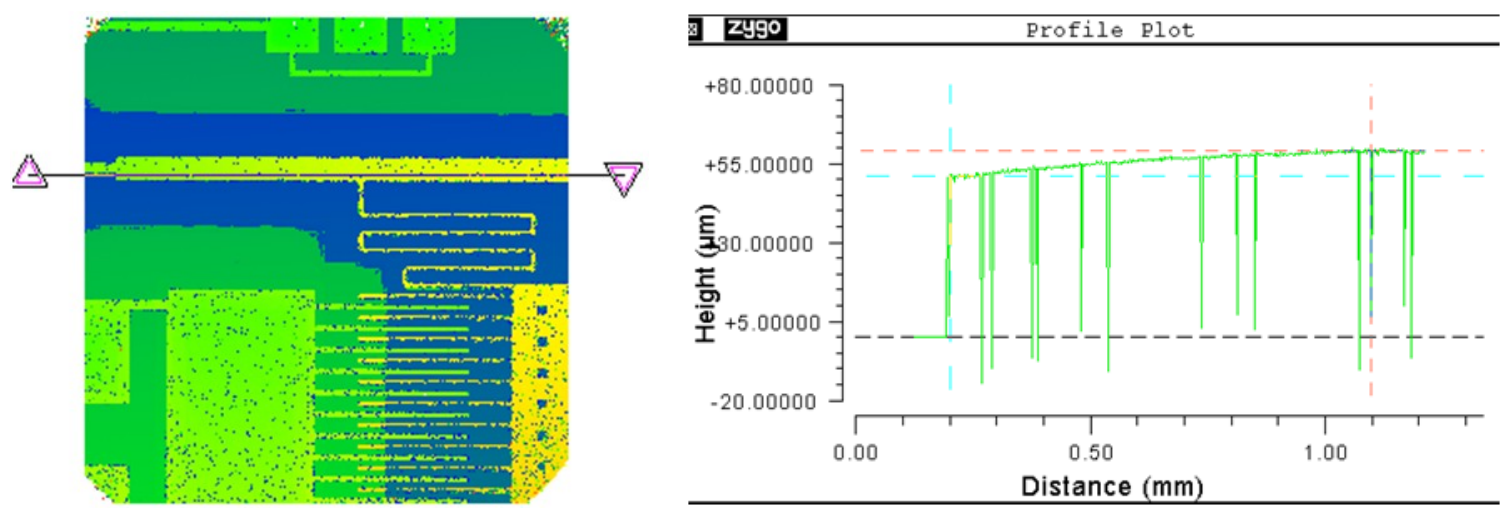

(c)

Figure C 2 Capacitor C3 (a) surface map and (b) profile plot (c) curve-up beam plot 


\section{ZY9O}

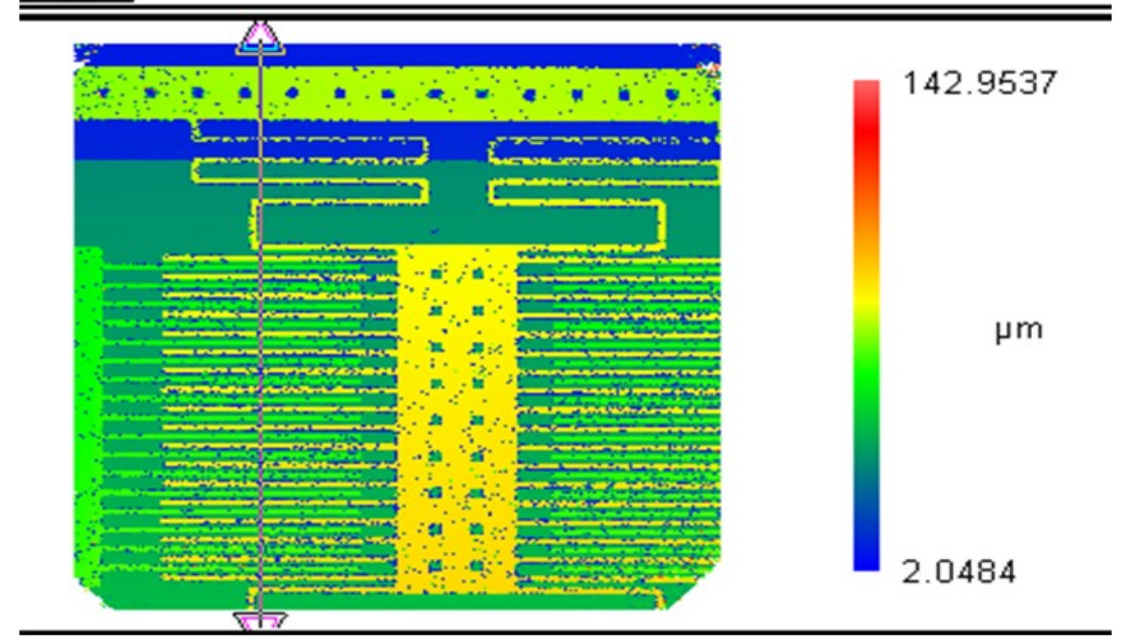

(a)

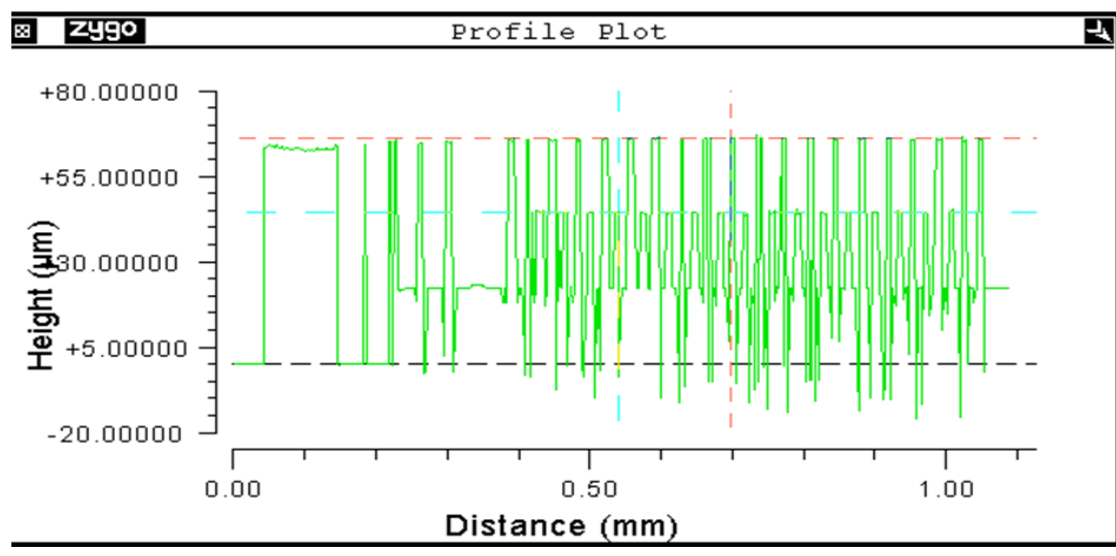

(b)
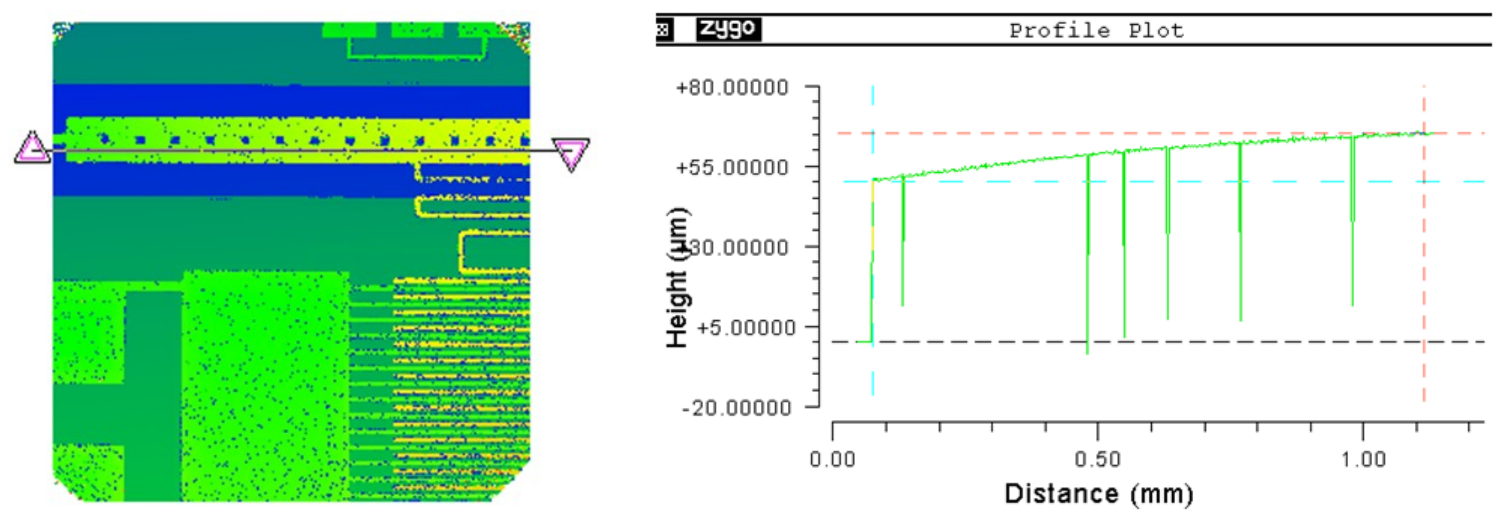

(c)

Figure C 3 Capacitor C4 (a) surface map and (b) profile plot (c) curve-up beam plot 


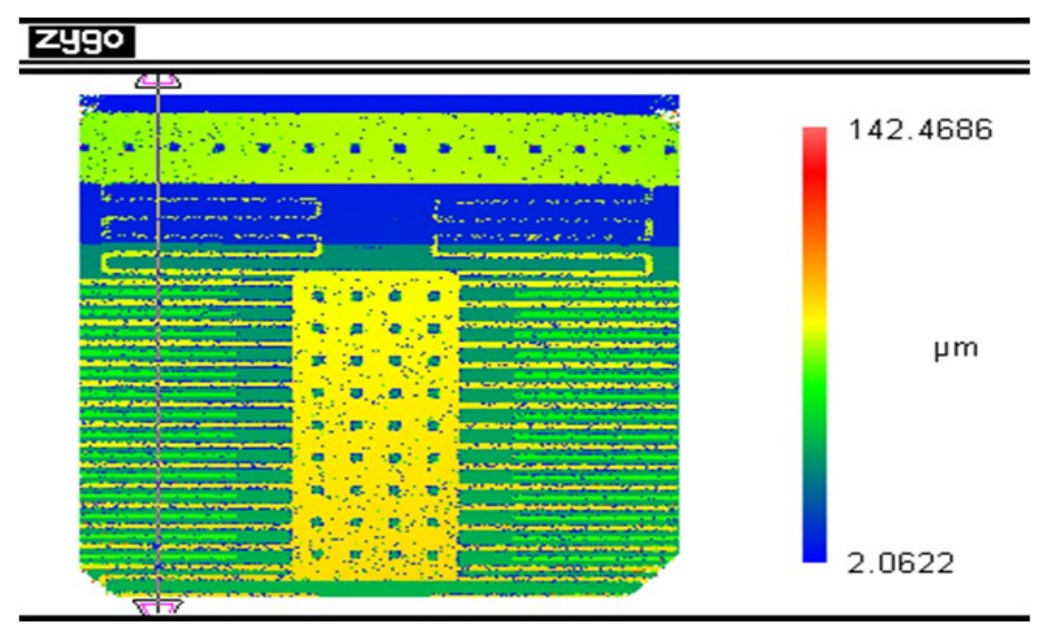

(a)

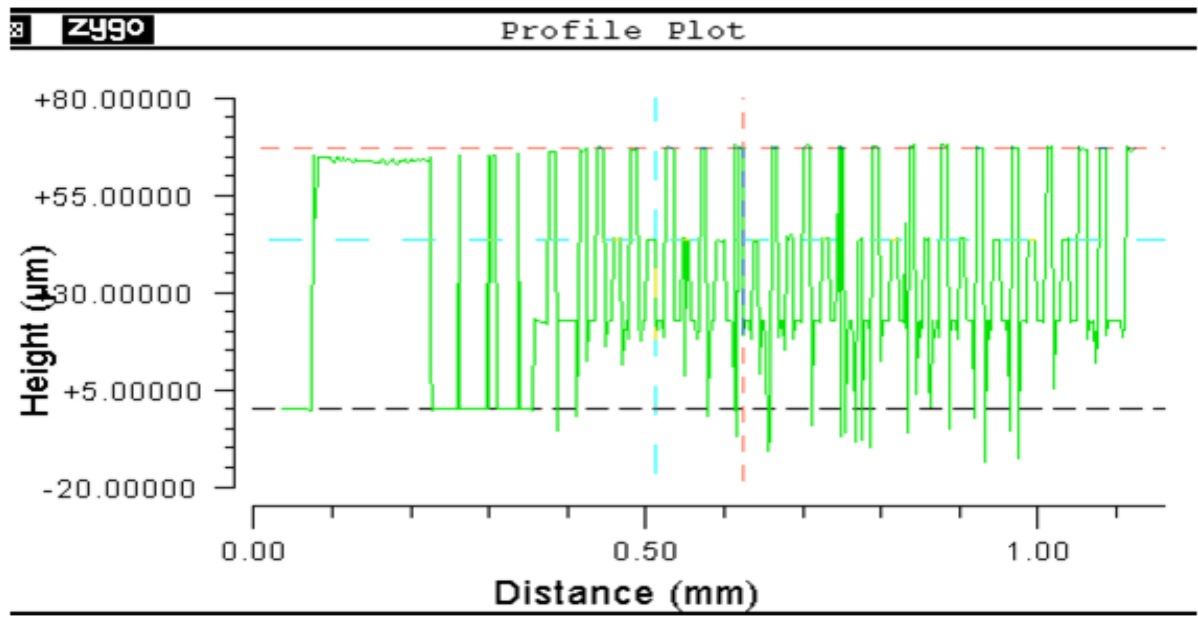

(b)
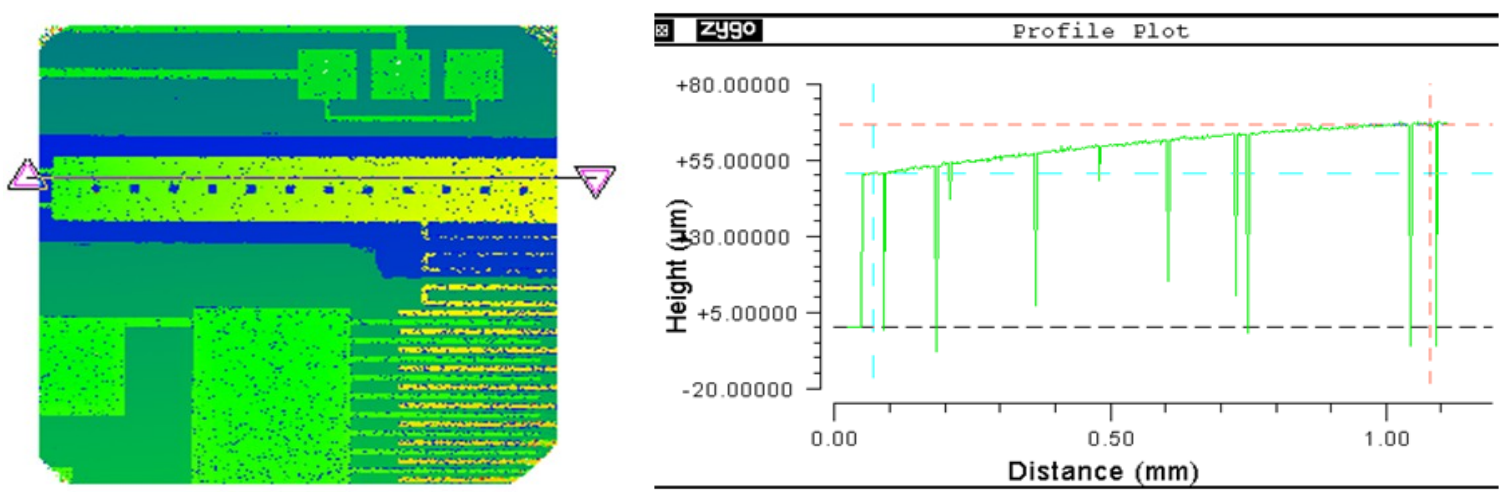

(c)

Figure C 4 Capacitor C6 (a) surface map and (b) profile plot (c) curve-up beam plot 


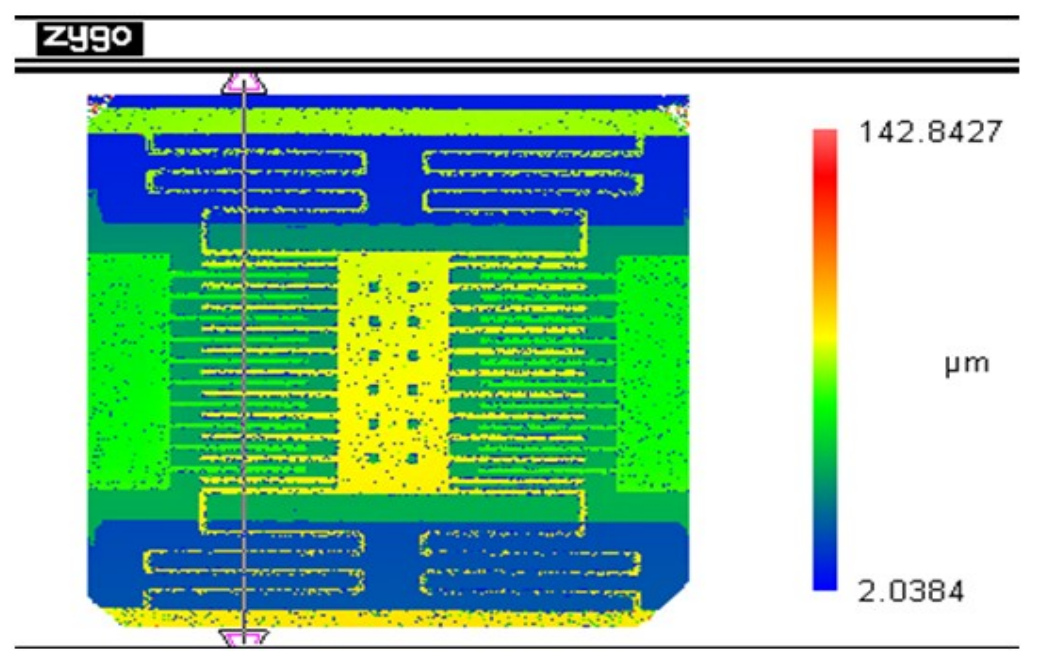

(a)

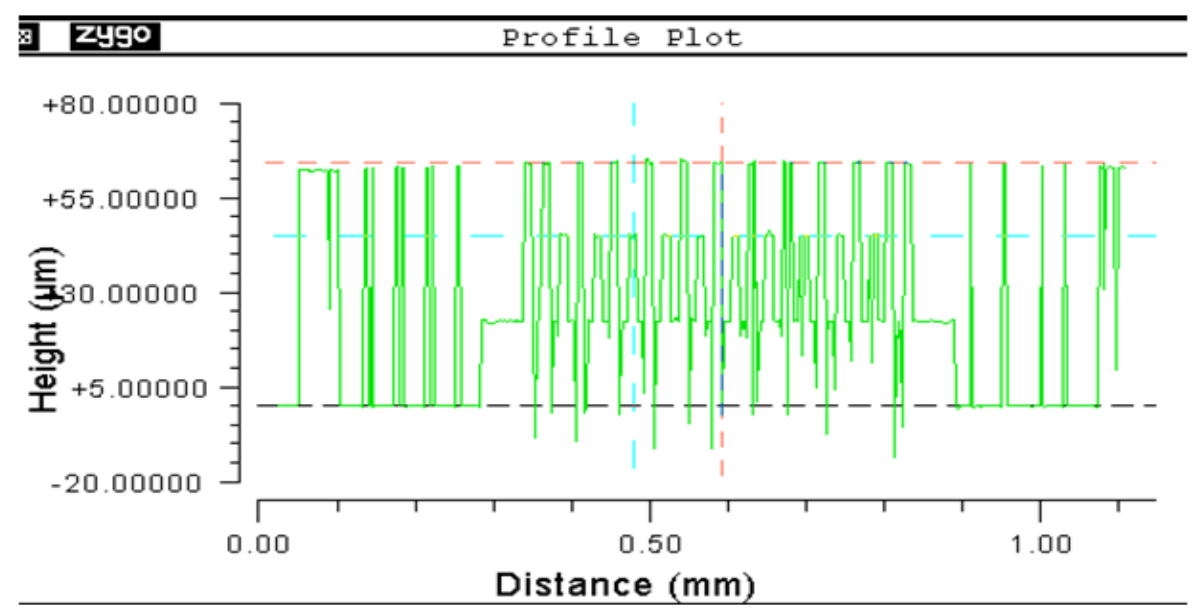

(b)
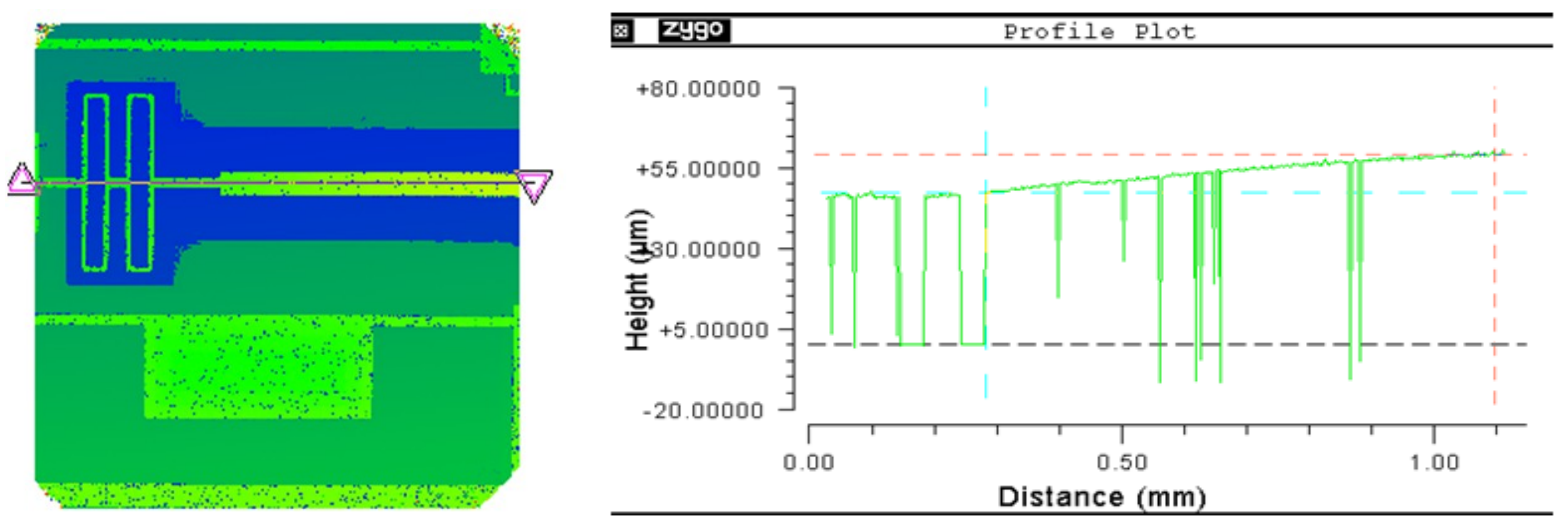

(c)

Figure C 5 Capacitor C8 (a) surface map and (b) profile plot (c) curve-up beam plot 


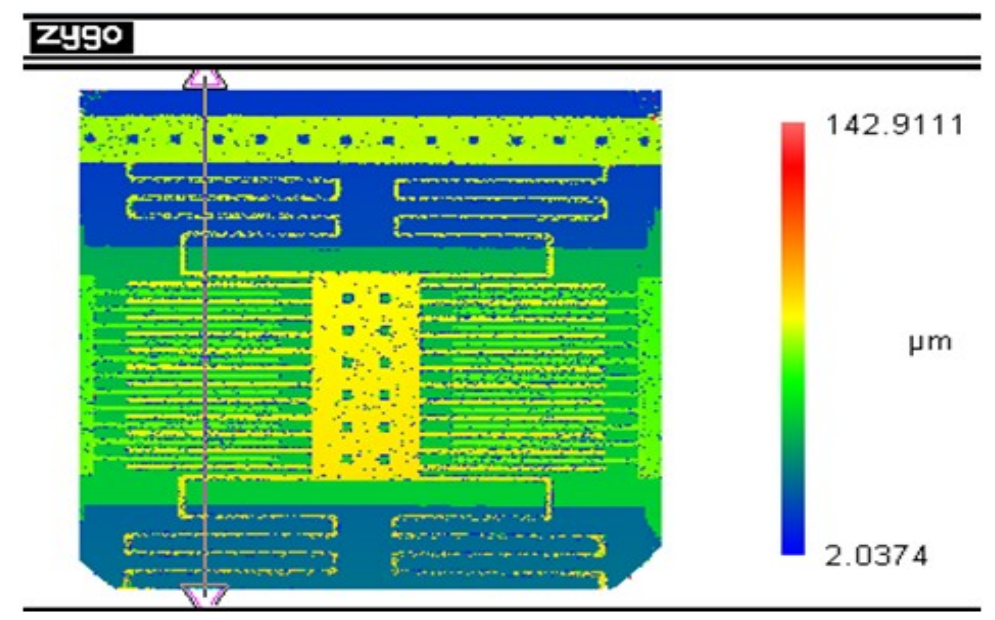

(a)

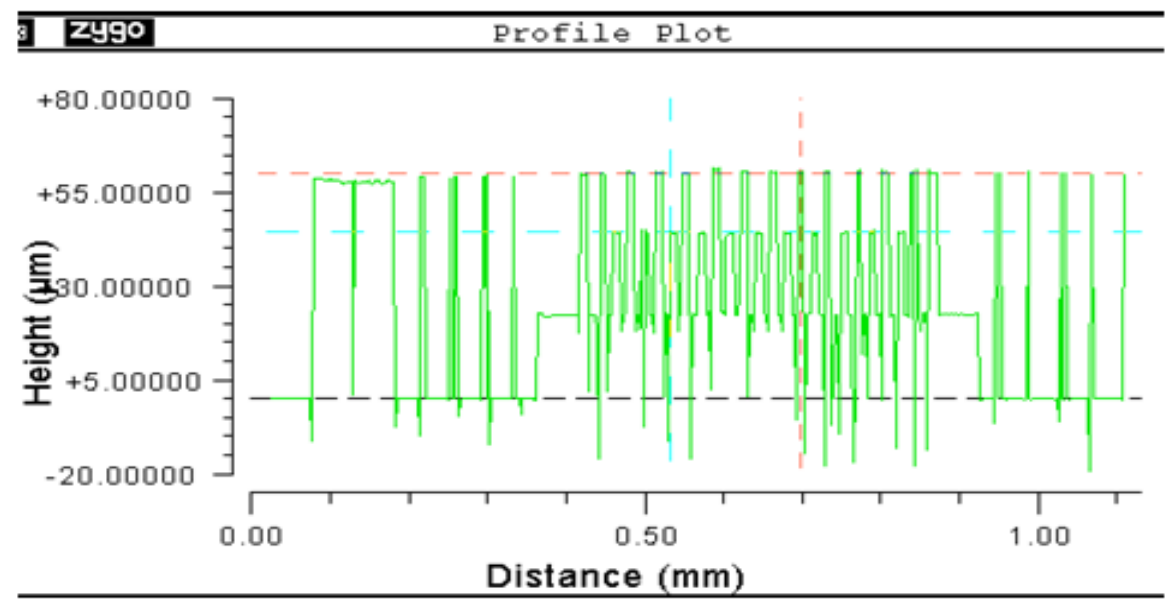

(b)
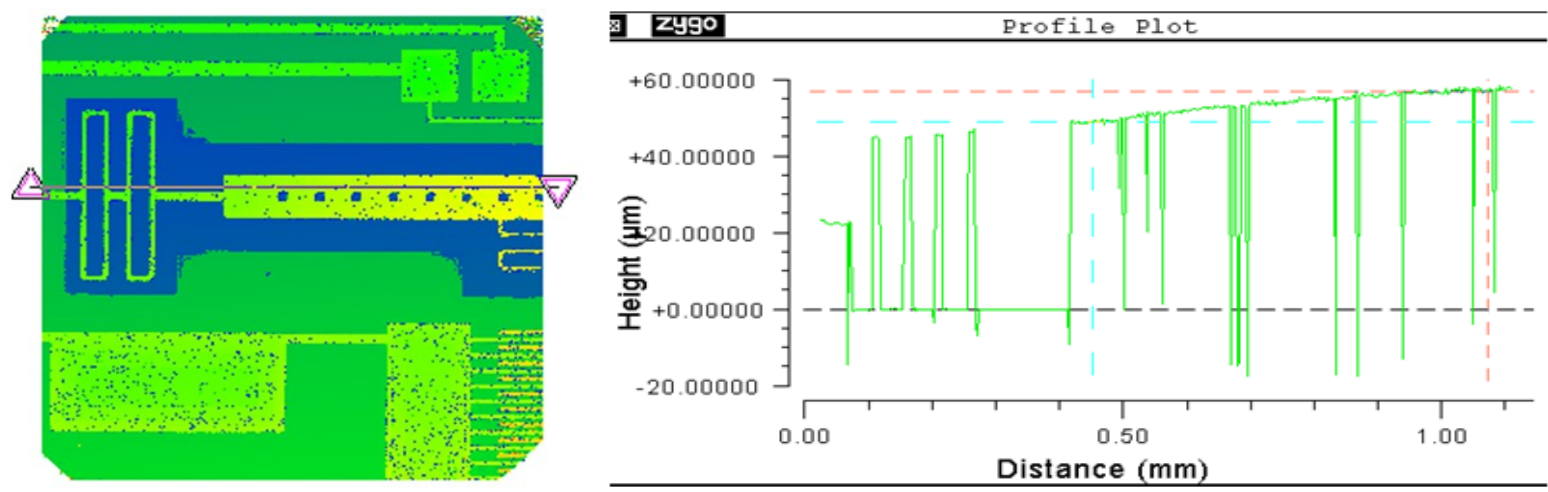

(c)

Figure C 6 Capacitor C9 (a) surface map and (b) profile plot (c) curve-up beam plot 


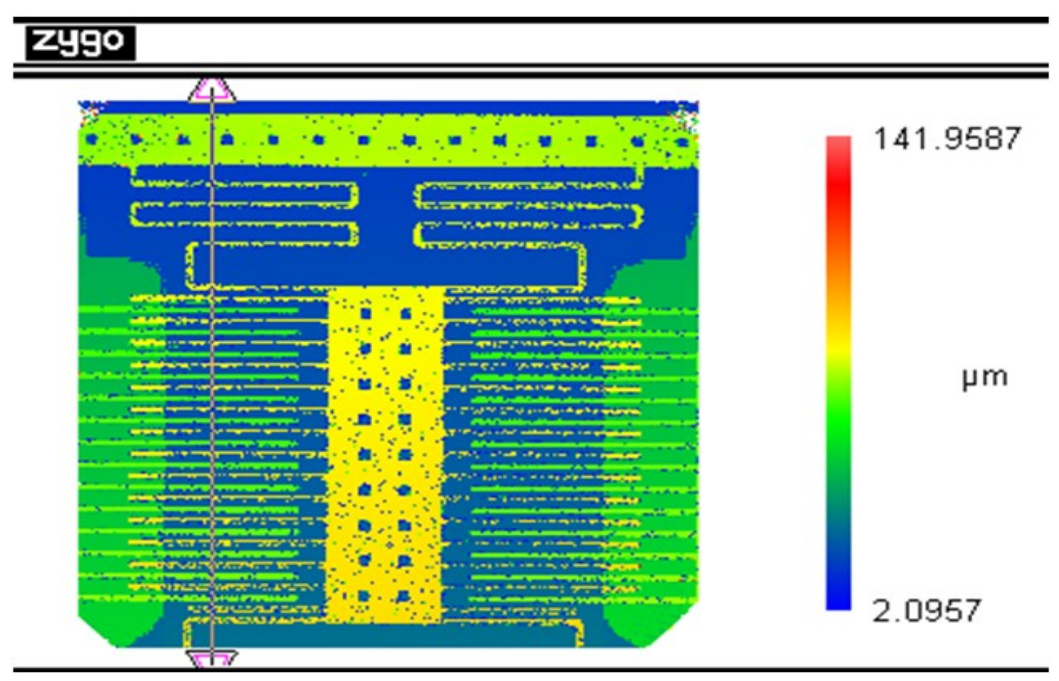

(a)

\begin{tabular}{ll}
\hline Z Zygo & Profile \\
\hline \hline
\end{tabular}

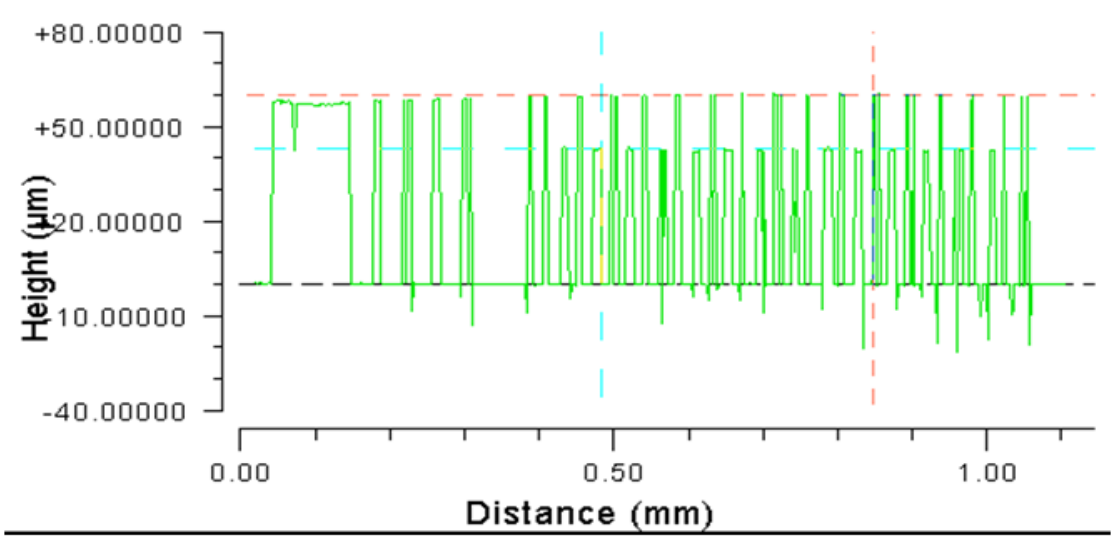

(b)

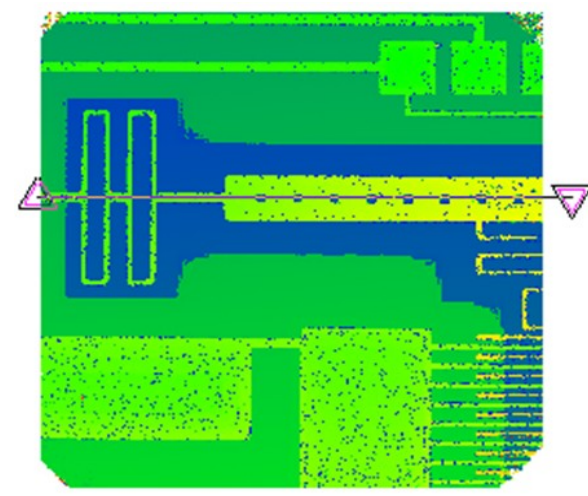

3. Zygo Profile Plot

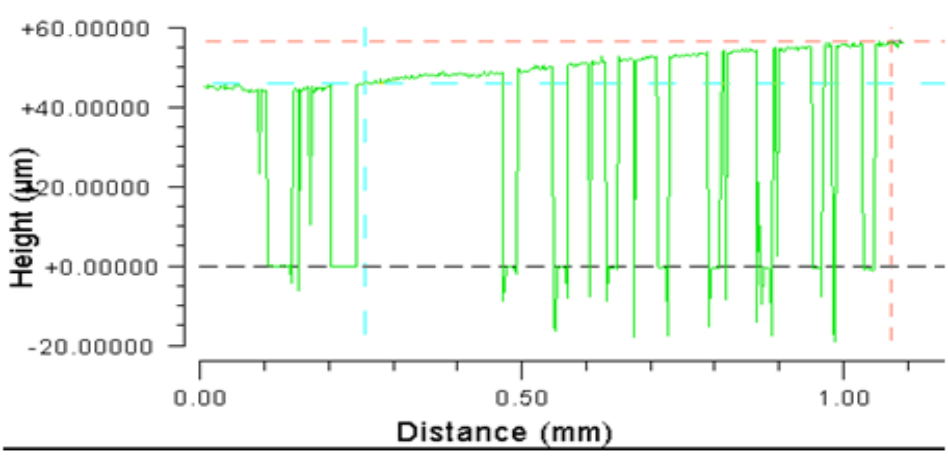

(c)

Figure C 7 Capacitor C10 (a) surface map and (b) profile plot (c) curve-up beam plot 


\section{Appendix D Matlab code to calculate capacitance and quality factor}

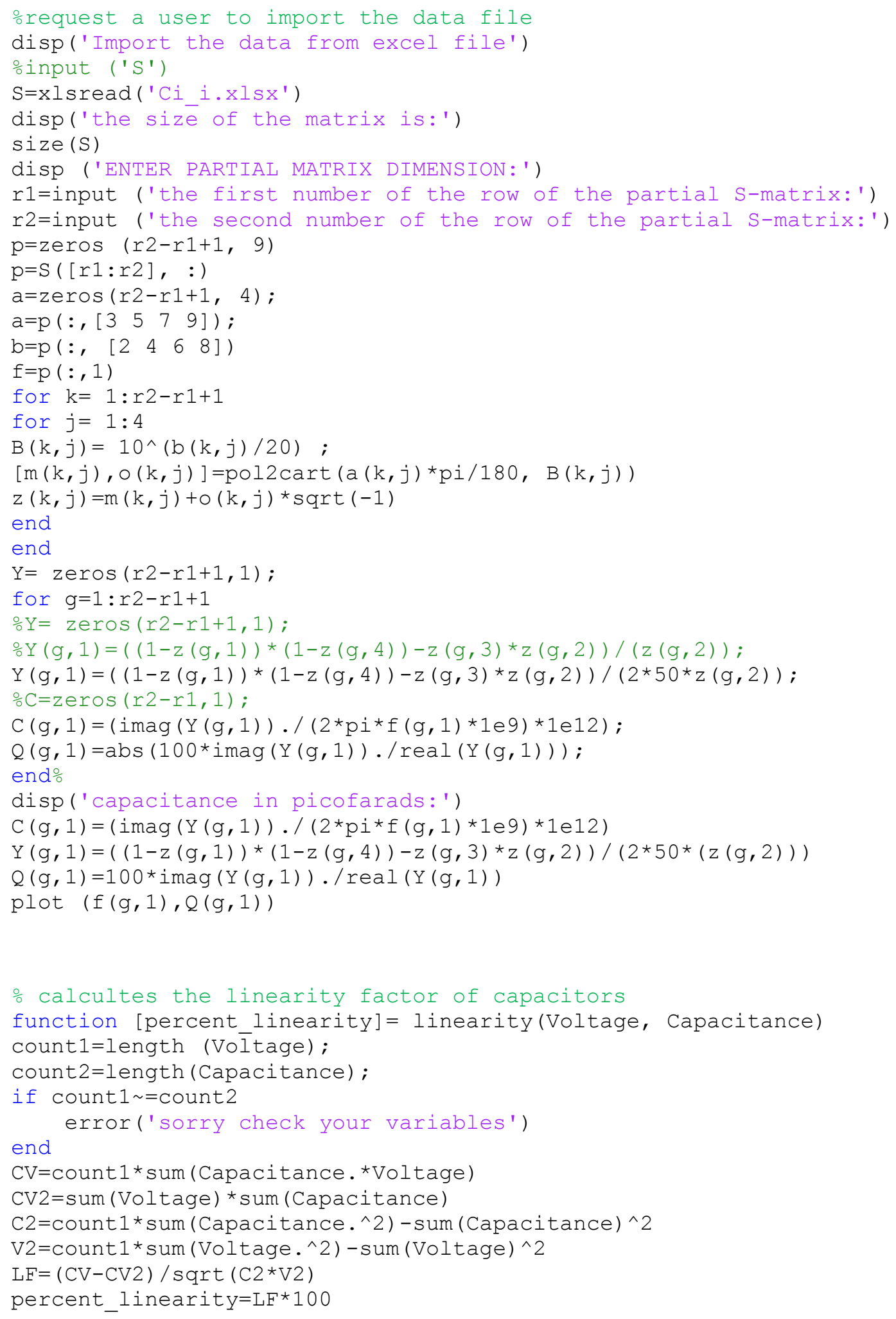




\section{Appendix E Quality factor plots}
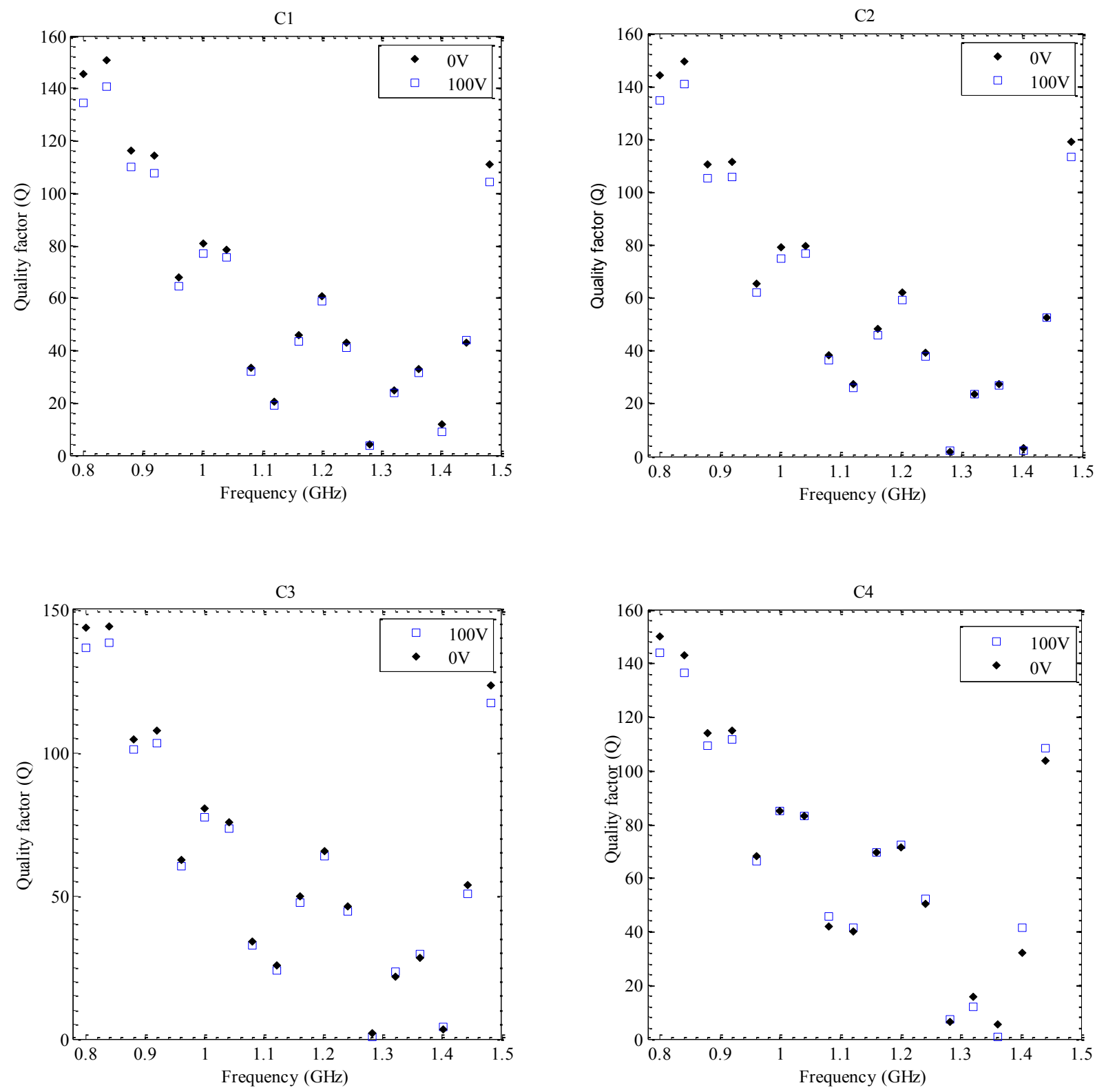

Figure E 1 Quality factor plots of C1-C4 

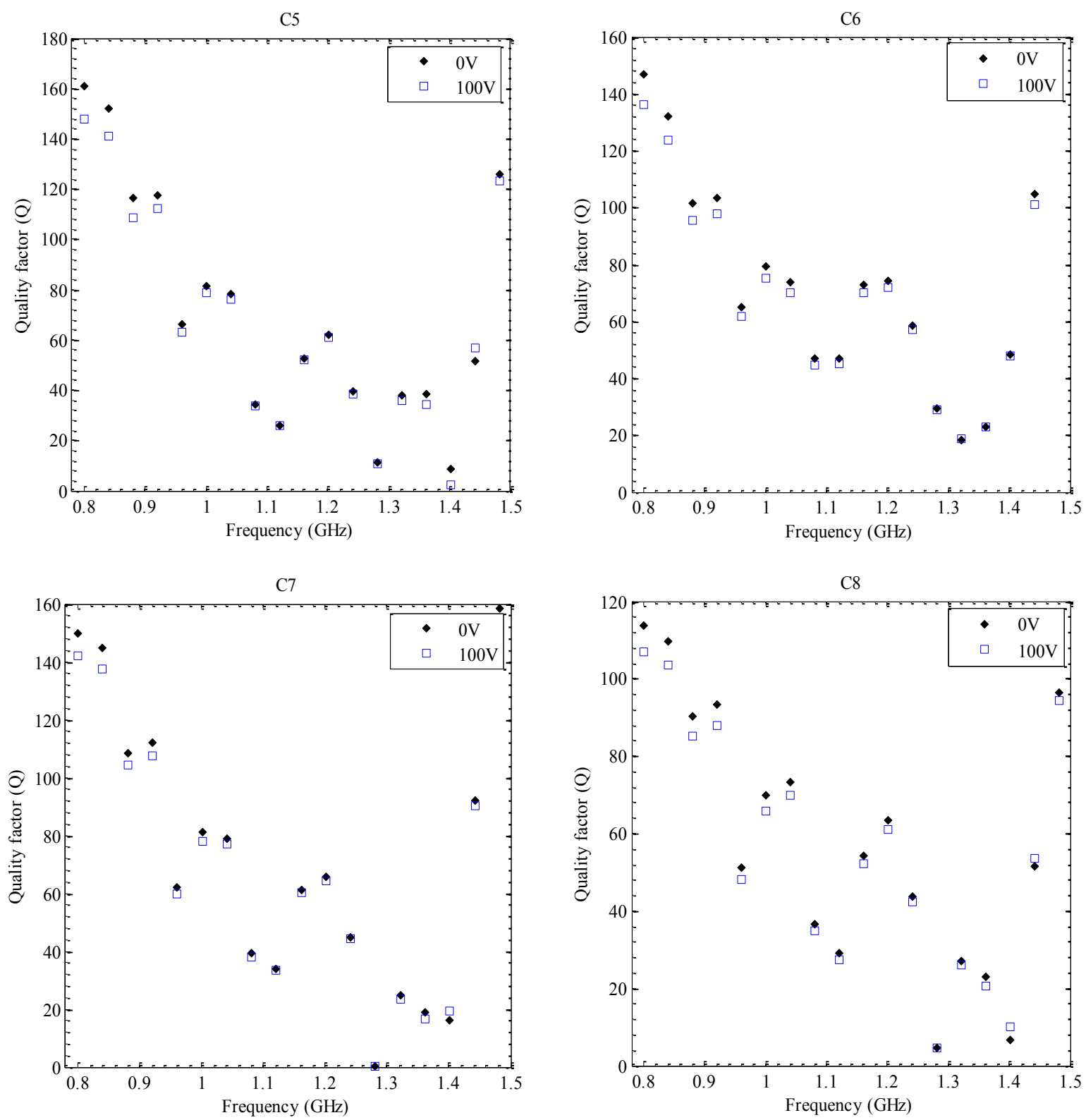

Figure E 2 Quality factor plots of C5-C8 

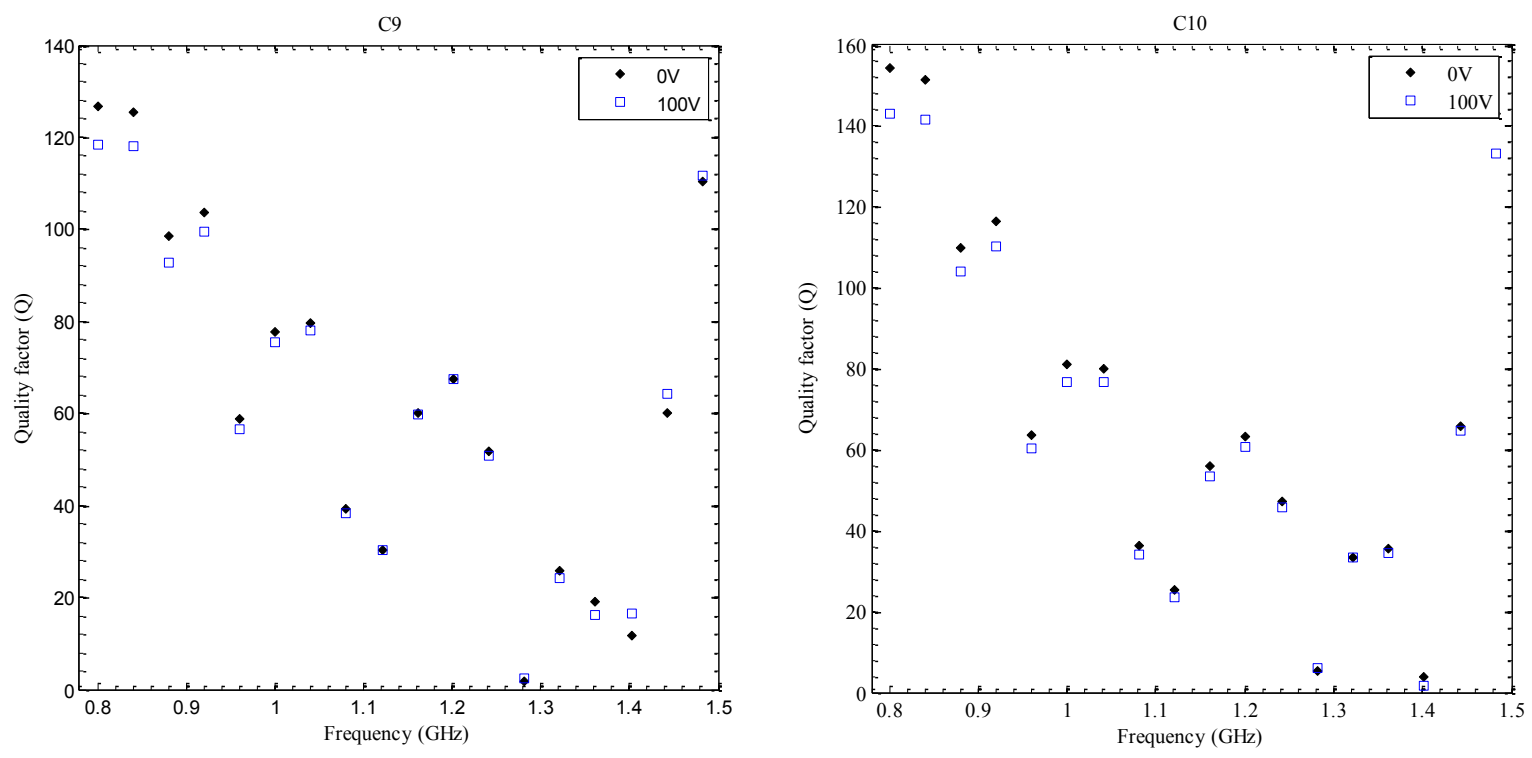

Figure E 3 Quality factor plots of C9 and C10 


\section{Appendix F Gap increasing tunable capacitor using gold fabrication process}

This section briefly explains the design and fabrication of unsuccessful repulsive electrostatic actuator driven tunable capacitor. These capacitors were fabricated using University of Waterloo MEMS microfabrication process. The process is has seven mask process that uses an alumina substrate as a starting material [1]. It consists of two dielectric films of silicon dioxide with thickness of $0.5 \mu \mathrm{m}$ and $0.7 \mu \mathrm{m}$ respectively. Two electroplated metallic layers, namely, Gold 1 and Gold 2 which are $1 \mu \mathrm{m}$ and $1.25 \mu \mathrm{m}$ were used as the fixed layer and the moving layers of the tunable capacitors. A polyimide sacrificial layer of thickness $2 \mu \mathrm{m}$ is removed by reactive ion etchant to leave the gap between the plates of the capacitor and the actuators. The 3D image of the capacitors is shown in Figure F 1.

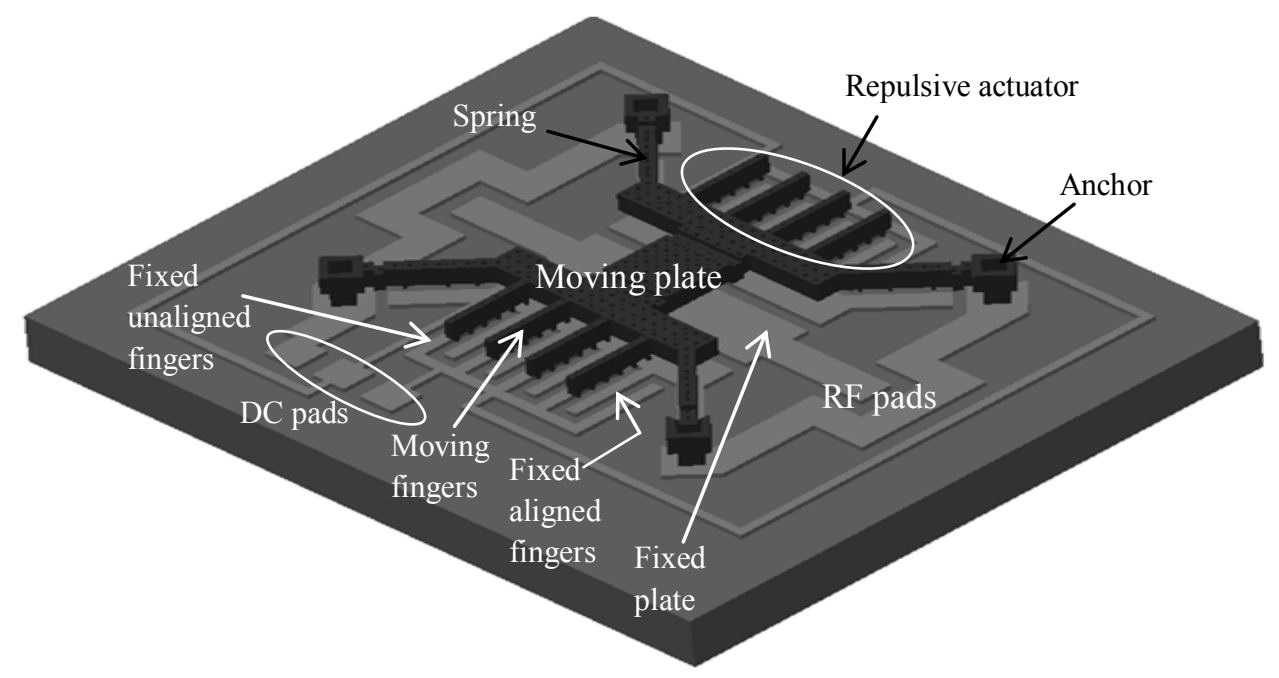

Figure F 1 D view of repulsive actuator driven tunable capacitor

However, the prototypes fabricated had residual stress gradient deformation that completely curved the moving fingers out of plane and upward. Due to this large distortion the repulsive actuation principle failed to work. Figure shows the 3D view of the actuator, surface map and profile plot of the actuator. The tips of the moving fingers are deflected by about $45 \mu \mathrm{m}$ above the fixed aligned fingers. As voltages up to $200 \mathrm{~V}$ are applied to the actuators' moving fingers and the moving plate no-change of displacement was measured. Figure shows the close view of the moving fingers of the actuator. The profile plot through the outer finger of shows that the moving fingers are elevated by $45 \mu \mathrm{m}$ which is difficult to drive with a $200 \mathrm{~V}$ actuation. 


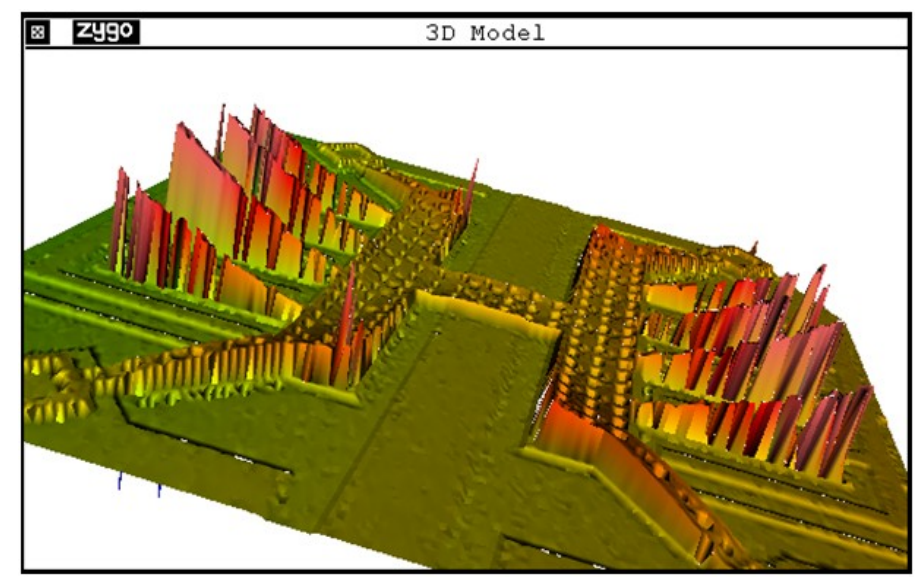

(a)

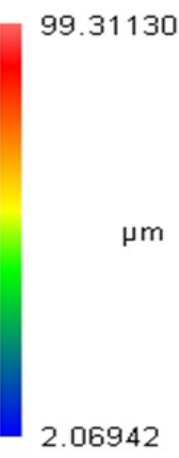

a)

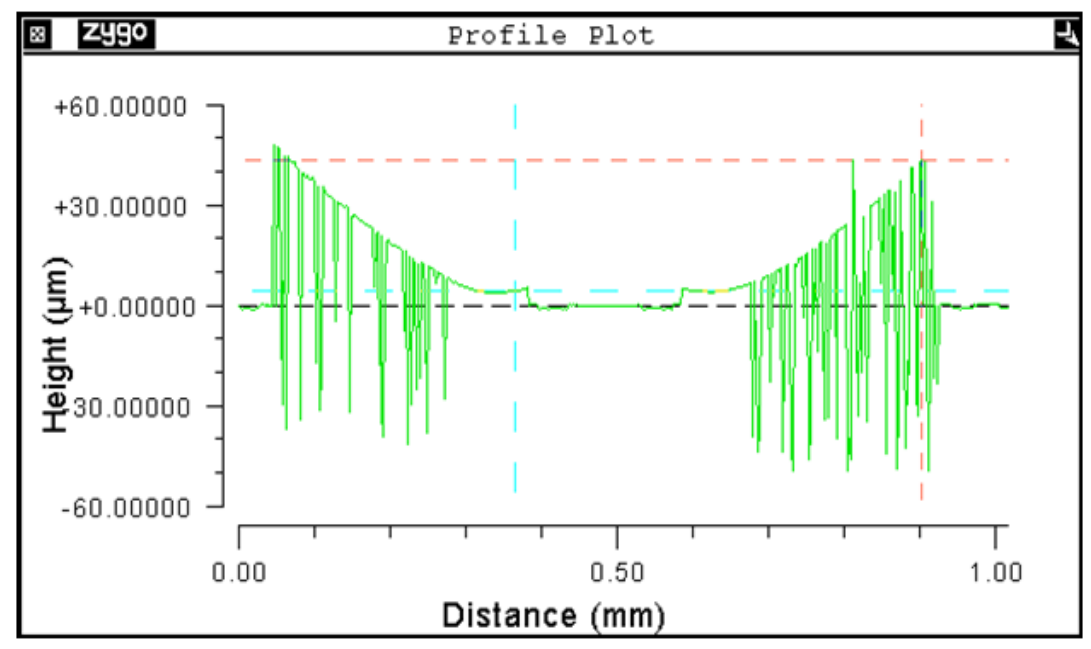

(b)

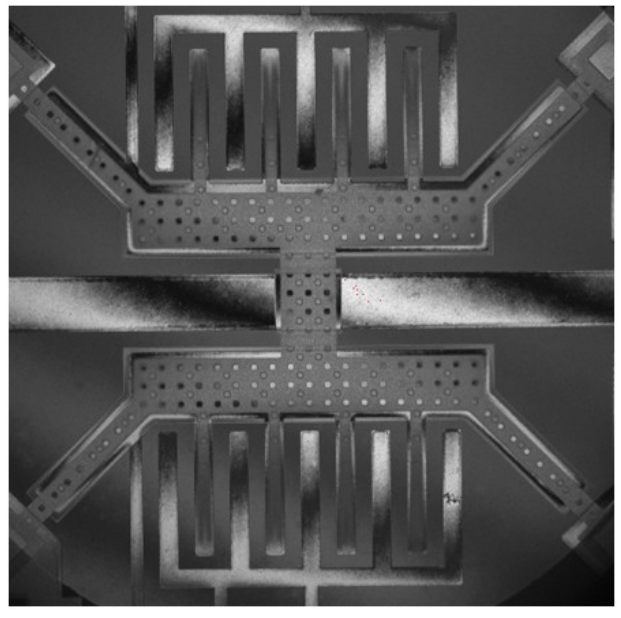

(d)

Figure F 2 Capacitor actuator (a) surface map, (b) profile plot (c) 3D model and (d) SEM image 


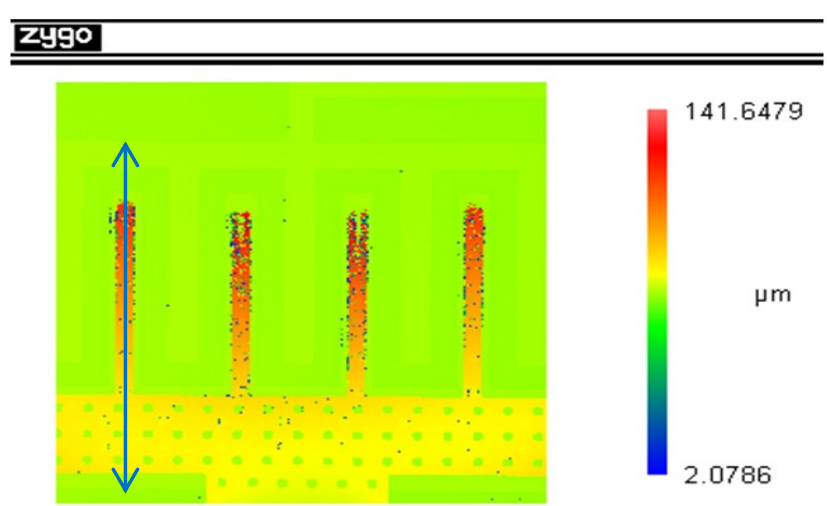

(a)

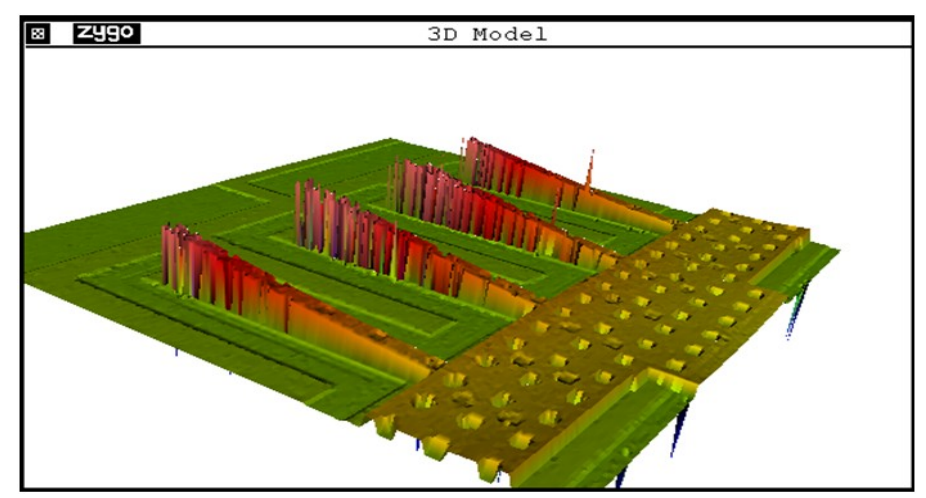

(b)

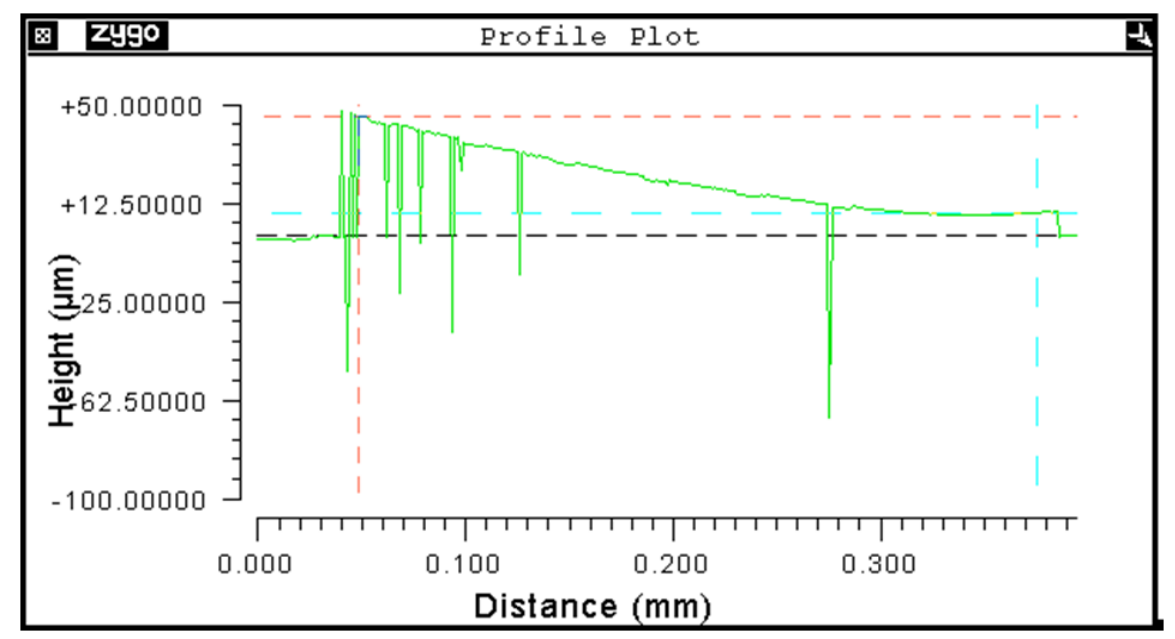

(c)

Figure F 3 Actuator moving finger (a) surface map, (b) 3D model and (c) profile plot 


\section{References}

[1] S. Shirota et al., "New type of varactor diode having strongly non-linear C-V characteristics", Electronic letters, Vol. 13 June 1977, pp 107-110

[2] S. M. Sze and K. K. Ng, "Physics of Semiconductor Devices", Ch 2, Wiley and Sons Inc., New York 2007

[3] M. P. J. Tiggelman, K. Reimann, F. Van Rijs, J. Smith and R. J. E. Hueting, "On the trade-off between quality factor and tuning ratio in tunable high frequency capacitors", IEEE Transactions on Electron Devices, Vol. 56, No. 9, September 2009, pp 2128-2136

[4] G. M. Rebeiz et al., "Tuning into RF MEMS", IEEE Microwave Magazine, Vol. 10. Issue 6, Topics: Field, Waves and Electromagnetics, October 2009, pp 55-72

[5] S. J. Park, "High-Performance RF-MEMS Tunable Filters", PhD thesis, Electrical Engineering, University of Michigan, 2008

[6] P. Sjöblom and H. Sjöland, "Measured CMOS switched high-quality capacitors in a reconfigurable matching network," IEEE Trans. Circuits Syst. II, Exp. Briefs , vol. 54, no. 10 , pp. 858-862, Oct. 2007.

[7] K. Yamamoto, T. Heima, A. Furukawa, M. Ono, Y. Hashizume, H. Komurasaki, S. Maeda, H. Sato, and N. Kato, "A 2. 4-GHz-band 1. 8-V operation single-chip Si-CMOS T/R-MMIC front-end with a low insertion loss switch,” IEEE J. Solid-State Circuits, vol. 36, no. 8, pp. 1186-1197, Aug. 2001.

[8] T. McKay, M. Carroll, J. Costa, C. Iversen, D. Kerr, and Y. Remoundos, "Linear cellular antenna switch for highly-integrated SOI front-end," in Proc. IEEE Int. SOI Conf., Oct. 2007, pp. $125-126$

[9] D. Kelly, C. Brindle, C. Kemerling, and M. Stuber, "The state-of-the-art of silicon-onsapphire CMOS RF switches,” in Proc. CSIC Dig., Nov. 2005,pp. 200-203

[10] S. Makioka, Y. Anda, K. Miyatsuji, and D. Ueda, "Super self-aligned GaAs RF switch IC with $0.25 \mathrm{~dB}$ extremely low insertion loss for mobile communication systems," IEEE Trans. Electron Devices, vol. 48, no. 8, pp. 1510-1514, Aug. 2001

[11] F-J. Huangand and K. O, "A $0.5-\mu \mathrm{m}$ CMOS T/R switch for 900-MHz wireless applications," IEEE J. Solid-State Circuits, vol. 36, no. 3, pp. 486-492, March 2001

[12] "RF MEMS in mobile phones", www. Rfdesign.com, September 2005 
[13] L. Dussopt and G. M. Rebeiz, "Intermodulation distortion and power handling in RF MEMS switches, varators, and tunable filters," IEEE Trans. Microwave Theory \& Tech., vol. 51, no. 4, pp. 1247-1256, Apr. 2003

[14] J. J. Yao, "RF MEMS from a device perspective", Journal of Micromechanics and Micro engineering. 10, 2000, R9

[15] http://www.allaboutcircuits.com/vol 3/chpt_3/12.html

[16] G. M. Rebeiz, et al, "Tuning in to RF MEMS", IEEE Microwave magazine, October 2009, pp. 55-72

[17] S. J. Park, "High-Performance RF-MEMS Tunable Filters", PhD thesis, Electrical Engineering, University of Michigan, 2008

[18] "RF MEMS in mobile phones", www. Rfdesign.com, September 2005

[19] L. Dussopt and G. M. Rebeiz, "Intermodulation distortion and power handling in RF MEMS switches, varators, and tunable filters," IEEE Trans. Microwave Theory \& Tech., vol. 51, no. 4, pp. 1247-1256, Apr. 2003

[20] D. J. Young and B. E. Boser, "A micro-machined variable capacitor for monolithic lownoise VCOs," in Solid-state and actuator workshop, Hilton Head, June 1996, pp 86-89

[21] A. Dec, K. Suyama, "RF Micromachined varactor with wide tuning range", IEEE Radio Frequency Integrated Circuits (RFIC) Symposium, 1998 7-9 June, pp 309-312

[22] G. M. Rebeiz, RF MEMS, Theory Design and Technology, Wiley 2003

[23] S. Jung et al., "Micromachined frequency variable impedance tuners using resonant unit cells," in IEEE MTT-S International Microwave Symposium Digest, Phoenix, AZ, May 2001, pp 333-336

[24] J. Zou et al, "Development of Wide Tuning Range MEMS Tunable Capacitor for Wireless Communication Systems", Technical Digest, International Electron Devices Meeting, San Francisco, CA, USA, 2000, pp. 403-406 
[25] J. Ehmke, J. Brank, A. Malczewski, B. Pillans, S. Eshelman, J. Yao and C. Goldsmith, "RF MEMS devices: a brave new world for RF technology" IEEE Emerging Technologies Symposium 2000

[26] N. Hoivik et al., "Digitally controllable variable high-Q MEMS capacitor for RF applications," in IEEE MTT-S International Microwave Symposium Digest, Phoenix, AZ, May 2001, pp 2115-2118

[27] F. Faheem, N. Hoivik, Y. Lee, and K. Gupta, "Post-enabled precision flip-chip assembly for variable mems capacitor," in Microwave Symposium Digest, 2003 IEEE MTT-S International, Vol. 3, 8-13 June 2003, pp. 1927-1930

[28] G. V. Ionis, A. Dec and K. Suyaman, "Differential multi-finger MEMS tunable capacitors for RF Integrated Circuits," IEEE MTT-S International Microwave Symposium Digest1, 2002 pp 345-348

[29] S. Seok, W. Choi and K. Chun, "A Novel linearly tunable MEMS variable capacitor", Journal of Micromechanics and Microengineering, January 2002, 12, pp 82-86

[30] R. L. Borwick et al., "A high Q, large tuning MEMS capacitor for RF Filter systems", Sensors and Actuators A103, 2003, pp 33-41

[31] R. L. Borwick et al, "Variable MEMS capacitors implemented into RF filter systems" IEEE Transaction Microwve Theory and Technology, January 2003 pp 315-319

[32] J.B. Yoon and C. T.C. Nguyen, "A high-Q tunable micromechanical capacitor with movable dielectric for RF applications," Technical Digest, IEEE Int. Electron Devices Meeting, San Francisco, California, Dec. 11-13, 2000, pp. 489-492

[33] R. Fritschi et al., "High tuning range AlSi RF MEMS capacitors fabricated with sacrificial amorphous silicon surface micromachining", Microelectronic Engineering 7374 March 2004, pp 447-451

[34] Rijks et al., "MEMS Tunable Capacitors and Switches for RF Applications", Proceedings of 24th International Conference on Microelectronics, 16-19 May 2004, Serbia and Montenegro, pp 49-56 
[35] M. Baker-Kassem and R. R. Mansour, "Two Movable Plate Nitride Loaded MEMS Tunable Capacitor", IEEE MIT-S Digest, 2004, pp 483-486

[36] A. Grichener, D. Mercier and G. M. Rebeiz, "High-Power, High-Reliability, High-Q Switched RF MEMS Capacitors," International Microwave Symposium Digest, 2006, pp 31-34

[37] Ching-Liang Daia et al., "Fabrication and characterization of a micro-electromechanical tunable capacitor", Microelectronics Journal 38, 2007, pp 1257-1262

[38] J.A. Etxeberria and F.J. Gracia,"High Q factor RF MEMS tunable Metallic Parallel Plate Capacitor", Electronics devices, Spanish Conference on Jan 31, 2007, pp 201-204

[39] Y. J. Yoon, H. S. Lee and J. Yoon, "MEMS Variable Capacitor actuated with electrically floating plate", IEEE International Electronic Devices meeting, IEDM 2007, 2007, pp 431-434

[40] G. McFeetors and M. Okoniewski, "Performance and Operation of Stressed Dual-gap RF MEMS Varactors", Proceedings of the 36th European Microwave Conference, September 2006, Manchester UK, pp 1064-1067

[41] Y. Zhu, M. R. Yuce, S. O. R. Moheimani, "A Low-Loss MEMS Tunable Capacitor with Movable Dielectric," IEEE SENSORS 2009 Conference, 2009, pp 651-654

[42] N. Zahirovic, R. R. Mansour and M. Yu, "A Three Step High-Q Variable MEMS Capacitor with low actuation voltage", Conference Proceedings European Microwave Week 2009, 2009, Article no 5296143, pp 1136-1139

[43] Q. Liu, "Laterally driven tunable capacitors fabricated by a surface micromachining process for RF circuits" 2nd Pacific-Asia Conference on Circuits, Communications and System, art. No. 5626961,2010, pp. 406-409

[44] M. Shavezipur, S.M Hashemi, P. Nieva, A. Khajepour," Development of a triangularplate MEMS tunable capacitor with linear capacitance-voltage response," 2010 Microelectronic Engineering 87 (9), 2010 , pp 1721-1727

[45] F. Barriere, A. Crunteanu, A. Pothier, M. Chatras, P. Blondy, "A low value normally on RF-MEMS switched capacitor for high Q millimeter wave tuning," $10^{\text {th }}$ Topical Meeting 
on Silicon Monolithic Integrated Circuits in RF Systems, SiRF 2010 - Digest of Papers, art. No. 5422958, pp. 77-79

[46] A. Grichener, G. M. Rebeiz, "High-reliability RF-MEMS switched capacitors with digital and analog tuning characteristics" 2010 IEEE Transactions on Microwave Theory and Techniques 58 (10), Article. No. 5575376, pp. 2692-2701

[47] R. Mahameed, M.A. El-Tanani, G. M. Rebeiz, "A zipper RF MEMS tunable capacitor with interdigitated RF and actuation electrodes" 2010 Journal of Micromechanics and Microengineering 20 (3), Article No. 035014

[48] H. Yamazaki et al, " A High Power-Handling RF MEMS Tunable Capacitor Using Quadruple Series Capacitor Structure ", International Microwave Symposium Digest (MTT), 23-28 May 2010, pp 1138-1141

[49] J. J. Allen, 2005, Micro Electro Mechanical Systems Design, 6000 Broken Sound Parkway NW, Suite 300, Taylor \& Francis Group

[50] R. Ghodssi and P. Lin, 2011, MEMS Materials and Processes Handbook, LLC, 233 Spring Street, New York,NY 10013, USA, Springer Science + Business Media

[51] V. K. Varadan, K. J. Vinoy and K.A. Jose, 2003, RF MEMS and their Applications, The Atrium, Southern Gate, Chichester,West Sussex PO19 8SQ, England, John Wiley \& Sons Ltd

[52] O. Gomis-Bellmunt and L.F. Campanile, 2010, Design Rules for Actuators in Active Mechanical Sytems, Springer

[53] C. Liu, 2006, Foundations of MEMS, Upper Saddle River, New Jersey 07458, Pearson Education, Inc.

[54] G. M. Rebeiz, 2003, RF MEMS Theory, Design and Technology, Hoboken New Jersey, John Wiley and Sons, Inc.

[55] X. Yang, C. Grosjean, and Y.-C. Tai, "Design, Fabrication, and Testing of Micromachined Silicone Rubber Membrane Valves", Journal of Micro-electromechanical Systems, 1999, 8(4), pp 393-402

[56] L. Que et al., "Bent Beam Electro-thermal Actuators ", Journal of Microelectromechanical Systems, 2001, pp 31-36

[57] J. H. Corntois and V. M. Bright, "Applications for surface-rnicromachined poly-silicon thermal actuators and arrays ", 1997, Sensors and actuators, A 58 (1997) 19-25 
[58] B. Wagner, W. Benecke, G. Engelmann, and J. Simon, "Micro-actuators with moving magnets for linear, torsional or multi-axial motion," Sensors and Actuators, vol. A, no. 32, pp. 598-603, 1992

[59] J. W. Judy and R. S. Muller, "Magnetic micro-actuation of torsional polysilicon structures," Sensors and Actuators, A (Physical), 1996, Vol. A53, pp. 392-397

[60] C. Liu, and Y. W. Yi, "Micromachined Magnetic Actuators Using Electroplated Permalloy IEEE Transactions on Magnetics Vol. 35 no. 3, May 1999, pp 1976-1985

[61] A.C. Hartley, R.E. Miles, J. Corda, N. Dimitrakopoulos, "Large throw magnetic microactuator" Mechatronics 18 (2008) pp 459-465

[62] Cowen, Busbee Hardy, R. Mahadevan, and S. Wilcenski, Poly-MUMPs Design Handbook, Revision 13.0 MEMSCAP Inc. available at http://www.memscap.com/ data/assets/pdf file/0019/1729/PolyMUMPsDR-13-0.pdf, (accessed on Jan 22 2012)

[63] S. He and R. Ben Mrad, "Design, modeling, and demonstration of a MEMS repulsiveforce out-of plane electrostatic micro-actuator," IEEE/ASME Journal of Microelectromechanical Systems, Vol. 17, 2008, pp.532-547

[64] S. He and R. Ben Mrad, "Large-Stroke micro-electrostatic actuators for vertical translation of micro-mirrors used in adaptive optics," IEEE Transaction on Industrial Electronics, Vol. 52, 2005 pp. 974-983

[65] S. He, R. Ben Mrad and J. Chong, "Repulsive-Force out of Plane Large Stroke Translation Micro Electrostatic Actuator", Journal of Micromechanics Micro-engineering Systems, Volume 21, Issue 7, July 2011, Article number 075002

[66] W.H. Hayat, Engineering Electromagnetics, second edition, 2001 McGraw Companies

[67] Z. Hailu, S. He and R. Ben Mrad, "A repulsive force electrostatic actuated RF MEMS tunable capacitor", 24th Canadian Congress of Applied Mechanics, Saskatoon, SK, Canada, June 2-6 2013 Accepted

[68] http://www.coventor.com/products/coventorware/

[69] Y. Xiang, "The Electrostatic Capacitance of an Inclined Plate Capacitor", Journal of Electrostatics 64, 2006, pp 29-34

[70] E. Freitag and R. Busam, Complex Analysis, 2nd edition, 2009, Springer-Verlag Berlin Heidelberg

[71] J. Bak and D. J. Newman, Complex Analysis, 3rd edition, Springer Science + Business Media, LLC 1991, 1997, 2010 
[72] http://www.mathworks.com/products/matlab/R2012b

[73] A. Papoulis and S.U. Pillai, Probability, Random Variables and Stochastic Processes, 4th Ed. McGraw Hill 2002

[74] Electro* 2D/RS Electrostatic Simulator, Integrated Engineering Software, V63

[75] J.B. Muldavin, and G. M. Rebeiz, "High-Isolation CPW MEMS Shunt Switches", IEEE Transactions on Microwave Theory and Techniques, Vol. 48 No. 6 June 2000

[76] G. M. Rebeiz, RF MEMS Theory, Design, and Technology. New Jersey, John Wiley \& Sons, 2003

[77] D. Girbau, N. Otegi, L. Pradell, A. Lázaro, " A MEMS Capacitor with Improved RF Power Handling Capability ", Gallium Arsenide and Other Semiconductor Application Symposium, EGAAS 2005 European, 2005, pp 501-504

[78] L. Dussopt, and G. M. Rebeiz, "Inter-modulation Distortion and Power Handling in RF MEMS Switches, Varactors, and Tunable Filters", IEEE Transactions on Microwave Theory and Techniques, vol. 51, No. 4, April 2003 pp 1247-1256

[79] R. Mahameed, M.A. El-Tanani, G. M. Rebeiz, "A zipper RF MEMS tunable capacitor with interdigitated RF and actuation electrodes" 2010 Journal of Micromechanics and Microengineering 20 (3), Article No. 035014

[80] W. C. Tang, M. G. Lim and R. T. Howe, "Electrostatic Comb Drive Levitation and Control Method", Journals of Microelectromechanical Systems, Vol. 1, No. 4 December 1992, pp 170-178

[81] S. Greek and K. Hjor, "The effects of internal stresses on the precision of moveable structures for optical MEMS", Broadband Optical Networks and Technologies: An Emerging Reality/Optical MEMS Smart Pixels Organic Optics and Optoelectronics 1998 IEEE/LEOS Summer Topical Meetings, pp 11-12

[82] Kuo-Shen Chen, "Techniques in Residual stress measurement for MEMS and their Applications "Book Chapter in MEMS/NEMS Handbook Techniques and Applications, Springer Link, 20006 pp 264-345

[83] M. Ohring, The Material Science of Thin Films, A Division of Harcourt Brace and Company New York 525 B Street, Suite 1900. San Diego, California 1992

[84] C. V. Thompson and R. Carel, "Stress and Grain Growth in Thin Films", Journal of Mechanics of Physics and Solids, 1996, Vol. 44, No. 5, pp 657-573 
[85] V. A. Aksyuk, F. Pardo and D.J. Bishop, "Stress-induced Curvature Engineering in Surface Micro-machined", Proceedings of SPIE--the International Society for Optical Engineering, 1999 Vol.3680 Issue II pp 984-993

[86] R. Ghodssi and P. Lin, MEMS Materials and Processes Handbook, Springer Science and Business Media, 2010, pp 137-191

[87] W. D. Nix, "Mechanical Properties of Thin Films", Metallurgical Transactions, The Minerals, Metals, and Material Society, Vol. 20A, November 1989, pp 2217-2245

[88] K-S. Chen, MEMS/NEMS Handbook Techniques and Applications, Chapter 9: Techniques in residual stress measurement for MEMS and their applications, pp.12521328, Springer: US, 2006

[89] H.D. Espinosa, Y. Zhu, M. Fischer and J. Hutchinson, "An Experimental/Computational Approach to Identify Moduli and Residual Stress in MEMS Radio-Frequency Switches" 2003, Experimental Mechanics 43(3), pp 309-316

[90] F. Fachin, S. A. Nikles, J. Dugundji, B. L. Wardle, "Analytical extraction of residual stresses and gradients in MEMS structures with application to CMOS layered materials", Journal of Micromechanics and Micro engineering, 2011, 21(9), art. No. 095017

[91] H. Löwe, W. Ehrfeld, J. Schiewe: Micro-Electroforming of Miniaturized Devices for Chemical Applications, In J.W. Schultze et al. (Eds.): Electrochemical Microsystem Technologies, pp. 245-268 (Taylor \& Francis, New York, NY, 2002)

[92] W. Fang, A.J. Wickert, Determining mean and gradient residual stresses in thin films using micro machined cantilevers, Journal of Micromechanics and Micro-engineering 6 1996, pp 301-309

[93] W. Fang and J. A. Wickert, "Post Buckling of Micro-machined Beams", Journal of Micro-mechanics and Micro-engineering, Vol. 4 August 41994 pp 116-122

[94] W.-H. Chu, M. Mehregany, "A study of residual stress distribution through the thickness of p+ silicon films", IEEE Transactions on Electron Devices 40, 1993, pp 1245-1250

[95] F. Ericson, et al., "High-sensitivity surface micromachined structures for internal stress and stress gradient evaluation, Journal of Micromechanics and Micro-engineering (1997) pp 30-36

[96] Y. Zhang and Y. Zhao, "An effective method of determining the residual stress gradients in a micro-cantilever", Microsystem Technologies 12 (2006) pp 357-36 
[97] C.-W. Baek, Y.-K. Kim, Y. Ahn, Y.-H. Kim, "Measurement of the mechanical properties of electroplated gold thin films using micro machined beam structures", Sensors and Actuators A: Physical 117, 2005 pp 17-27

[98] A.B. Yu, et al., "Characterization and optimization of dry releasing for the fabrication of RF MEMS capacitive switches", Journal of Micromechanics and Micro-engineering 17 2007, pp 2024-2030

[99] E.H. Yang and S.S. Yang, The quantitative determination of the residual stress pro-file in oxidized p+ silicon films, Sensors and Actuators A: Physical 54 (1996) pp 684-689

[100] J.K. Luo, A.J. Flewitt, S.M. Spearing, N.A. Fleck, W.I. Milne, Young's modulus of electroplated Ni thin film for MEMS applications, Materials Letters 58 (2004) pp 23062309

[101] S. He, J. S. Chang, L. Li and H. Ho, "Characterization of Young's modulus and residual stress gradient of MetalMUMPs electroplated nickel film", Sensors and Actuators, 2009, Vol. 154 No 1, pp 149-156

[102] CoventorWa ${ }^{\text {reTM }}, 2008$, Coventor Inc http://www.coventor.com/products/coventorware/

[103] A. Cowen, R. Mahadevan, S. Johnson, and B. Hardy, (2002) Metal-MUMPs Design Handbook, Revision 3.0, MEMSCAP Inc., available at http://www.memscap.com/mumps/documents/MetalMUMPs.DR.2.2.pdf (accessed on June /01/2011)

[104] M. Bakri-Kassem, R.R. Mansour, Two movable-plate nitride-loaded MEMS vari-able capacitor, IEEE Transaction on Microwave Theory and Techniques 52 2004, pp 831-83

[105] L. Li, D. Uttamchandani, Monolithic RF MEMS inductor using silicon MEMS foundry process, Micro \& Nano Letters 12006 pp 5-8

[106] Imed Zine-El-Abidine, Michal Okoniewski, A tunable radio frequency MEMS inductor using MetalMUMPs, Journal of Micromechanics and Micro engineering 172007 pp $2280-228$

[107] D. Girbau, L. Pradell, A. Lazaro, A. Nebot, Electrothermally actuated RF MEMS switches suspended on a low-resistivity substrate, IEEE/ASME Journal of Microelectromechanical Systems 16 (2007) 1061-1070 
[108] M. Daneshmand, S. Fouladi, R. R. Mansour, M. Lisi and Tony Stajcer "ThermallyActuated Latching RF MEMS Switch”, Microwave Symposium Digest, 2009, MTT '09. IEEE MTT-S International, pp 1217-1220

[109] M. Daneshmand, W. D. Yan, and R. R. Mansour, "Thermally Actuated Multiport RF MEMS Switches and Their Performance in a Vacuumed Environment", IEEE Transactions on Microwave Theory and Techniques, Vol. 55, No. 6, JUNE 2007

[110] R. Ellis, Calculus with Analytic Geometry, $5^{\text {th }}$ edition, 1993

[111] David M. Pozar, Microwave Engineering, $4^{\text {th }}$ edition, Wiley and Sons Inc, 2005

[112] K.S. Chen, "Techniques in residual stress measurement for MEMS and their applications" MEMS/NEMS 2006, Springer Link

[113] W. Fang, and A.J. Wickert, "Determining mean and gradient residual stresses in thin films using micromachined cantilevers", Journal Micro-mechanics and Microengineering. 1996, 6, pp 301-309

[114] J. C. Chiou and Y. J. Lin, " A novel large displacement electrostatic actuator: pre-stress comb-drive actuator", Journal of Micromechanics and Microengineering, July 2005, pp $1641-1648$

[115] R. Legtenberg, A. W. Groeneveld and M. Elwenspoek, "Comb-Drive Actuators for large displacements", Journal of Micromechanics and Micro engineering, 1996, pp 320-329

[116] Gabriel M. Rebeiz, RF MEMS Theory, Design and Technology, John Wiley and Sons publication, 2003

[117] W. C. Tang, T. H. Nguyen, and R. T. Howe, "Laterally Driven Polysilicon Resonant Microstructures ", IEEE 1989, pp 53-59

[118] T. Hirano, T. Furuhata, K.J. Gabriel, and H. Fujita, "Design, Fabrication, and Operation of Gap Comb-Drive Micro-actuators", Journals of Microelectromechanical Systems, Vol. 1, No. 1, March 1992, pp 52-59

[119] W. C. Tang, M. G. Lim and R. T. Howe, "Electrostatic Comb Drive Levitation and Control Method", Journals of Microelectromechanical Systems, Vol. 1, No. 4 December 1992, pp 170-178

[120] R. Legtenberg, A. W. Groeneveld and M. Elwenspoek, "Comb-drive actuators for large displacements", Journal of Micromechanics and Micro engineering 6 1999, pp 320-329 
[121] A. P. Pisano and Y. H. Cho 1990 Mechanical design issues in laterally-driven microstructures Sensors Actuators A21- A23, 1990, pp 1060 - 1064

[122] R. Kondo, S. Takimoto, K. Suzuki, S. Sugiyama, "High aspect ratio electrostatic micro actuators using LIGA process", Microsystem Technologies 6 2000, pp 218-211

[123] C. S. B. Lee, S. Han and N. C. MacDona, "Single Crystal Silicon (SCS) XY-Stage Fabricated by DRIE and IR alignment ", 2000 IEEE, pp 28-33

[124] A. P. Lee, Charles F. McConaghy, G. Sommargren, P. Krulevitch, and E. W. Campbell, "Vertical-Actuated Electrostatic Comb Drive With In Situ Capacitive Position Correction for Application in Phase Shifting Diffraction Interferometry", Journal of Microelectromechanical Systems, Vol. 12, No. 6, December 2003, pp 960-971

[125] H. Xie and G.K. Fedder, "Vertical comb-finger capacitive actuation and sensing for CMOS MEMS", Sensors and Actuators A 95 2002, pp 212-221

[126] G. Krijnen, T. Kuijpers, T. Lammerink, R. Wiegerink and M. Elwenspoek, "Combdrives: Versatile micro-structures for capacitive sensing and electrostatic actuation", European Workshop on Smart Structures in Engineering and Technology, Brian Culshaw, Editor, Proceedings of SPIE Vol. 4763 (2003)

[127] J. D. Grade, H. Jerman, and T.s W. Kenny, " Design of Large Deflection Electrostatic Actuators ", Journal of Microelectromechanical Systems, Vol. 12, No.3, June 2003, pp 335-343

[128] P. R. Patterson et al, "A Scanning Micro-mirror with Angular Comb Drive Actuation", IEEE 2002, pp 544-547

[129] H. Nguyen et al, "Angular Vertical Comb-Driven Tunable Capacitor with High-Tuning Capabilities", Journal of Microelectromechanical Systems, Vol. 13, No.3, June 2004, pp 406-413

[130] Q. X. Zhang, A. Q. Liu, J. Li and A. B. Yu, "Fabrication technique for Microelectromechanical systems vertical comb-drive actuators on a monolithic silicon substrate", Journal of Vacuum Science and Technology B 23 32, 2005, pp 32-41

[131] A. Selvakumar and K. Najaf, "Vertical Comb Array Micro actuators", Journal of Microelectromechanical systems, Vol. 12, No. 4, August 2003, pp 440-449 
[132] O. Tsuboi et al, "A Rotational Comb-driven Micro mirror with a Large Deflection Angle and Low Drive Voltage", The Fifth IEEE International Conference on Microelectromechanical Systems 2002, pp. 532-535 